University of Tennessee Health Science Center UTHSC Digital Commons

$12-2021$

\title{
The Effect of Cancer Cachexia Progression on the Feeding Regulation of Skeletal Muscle Protein Turnover
}

Brittany R. Franch

Follow this and additional works at: https://dc.uthsc.edu/dissertations

Part of the Medical Cell Biology Commons, Medical Physiology Commons, Musculoskeletal, Neural, and Ocular Physiology Commons, and the Neoplasms Commons 


\title{
The Effect of Cancer Cachexia Progression on the Feeding Regulation of Skeletal Muscle Protein Turnover
}

\begin{abstract}
Cancer cachexia is defined as the unintentional loss of skeletal muscle mass with or without fat loss that cannot be reversed by conventional nutritional support. Cachexia occurs in $\sim 20 \%$ of cancer patients. More specifically, $50 \%$ of lung cancer patients, the most common cancer worldwide, develop cachexia. Cachexia occurs most often in lung and gastrointestinal cancers, whereas breast and prostate have the lowest rate of cachexia. Cancer-induced cachexia disrupts skeletal muscle protein turnover (decreasing protein synthesis and increasing protein degradation). Skeletal muscle's capacity for protein synthesis is highly sensitive to local and systemic stimuli that are controlled by mTORC1 and AMPK signaling. During cachexia, altered protein turnover is thought to occur through suppressed anabolic signaling via mTORC1, coinciding with the chronic activation of AMPK. While progress has been made in understanding some of the mechanisms underlying the suppressed anabolic signaling in cachectic muscle, gaps still remain in our understanding of muscle's ability to respond to anabolic stimulus prior to cachexia development. The purpose of this study was to determine if cachexia progression disrupts the feeding regulation of AMPK signaling and if gp130 signaling and muscle contraction could regulate this process.
\end{abstract}

Specific aim 1 examined the feeding regulation of skeletal muscle protein synthesis in pre-cachectic tumor bearing mice. Feeding increased muscle protein synthesis, while lowering AMPK signaling in precachectic tumor bearing mice. Importantly, pre-cachectic tumor bearing mice have overall suppressed muscle protein synthesis independent of the fast or fed condition. Muscle specific AMPK loss was sufficient to improve the fasting suppression of muscle mTORC1 and protein synthesis in pre-cachectic tumor bearing mice. Specific aim 2 examined if muscle gp130 signaling regulates the feeding regulation of AMPK during cancer cachexia progression. Muscle gp130 loss lowered the fasting induction of AMPK in pre-cachectic tumor bearing mice without improving protein synthesis. Muscle gp130 loss did not alter the feeding regulation of muscle Akt/mTORC1 signaling and protein synthesis. Specific Aim 3 examined if an acute bout of muscle contractions could improve the muscle protein synthesis response to feeding during the progression of cachexia. Pre-cachectic tumor bearing mice exhibit suppressed protein synthesis in response low frequency electrical stimulation, and the inability to synergistically induce protein synthesis in response to feeding and contraction.

In summary, pre-cachectic tumor bearing mice have lowered Akt/mTORC1 signaling and protein synthesis. Feeding can induce Akt/mTORC1 and protein synthesis and AMPK regulates the fasting suppression of protein synthesis in pre-cachectic tumor bearing mice. While gp130 loss reduces AMPK it is not sufficient to improve protein synthesis in pre-cachectic tumor bearing mice. The added protein synthesis response to feeding and contraction is blunted in pre-cachectic tumor bearing mice. These findings provide novel insight into the regulation of Akt/mTORC1 signaling and protein synthesis in response to feeding. Additionally, these studies highlight gp130's regulation of AMPK prior to cachexia development, and the blunted anabolic muscle response to feeding and contraction in pre-cachectic tumor bearing mice. By understanding these intracellular signaling processes and perturbations prior to cachexia development, we will be able to elucidate potential therapeutic targets and treatment options to manipulate and prevent cancer cachexia.

\section{Document Type}

Dissertation

\author{
Degree Name \\ Doctor of Philosophy (PhD)
}




\section{Program}

Biomedical Sciences

Research Advisor

James A. Carson, PhD

\section{Subject Categories}

Diseases | Medical Cell Biology | Medical Physiology | Medical Sciences | Medicine and Health Sciences | Musculoskeletal, Neural, and Ocular Physiology | Neoplasms 
DOCTORAL DISSERTATION

\section{The Effect of Cancer Cachexia Progression on the Feeding Regulation of Skeletal Muscle Protein Turnover}

Author:

Brittany R. Franch
Advisor:

James A. Carson, Ph.D.

A Dissertation Presented for The Graduate Studies Council of

The University of Tennessee Health Science Center

in Partial Fulfillment of the Requirements for the Doctor of Philosophy degree from

The University of Tennessee

in

Biomedical Sciences: Rehabilitation Sciences

College of Graduate Health Sciences

December 2021 
Copyright (C) 2021 by Brittany R. Franch. All rights reserved. 


\section{DEDICATION}

I'd like to thank my family, friends, and loved ones who have been there

for me every step of the way. Your continual support

has helped me get to where I am today.

I will be forever thankful for all of their support. 


\section{ACKNOWLEDGEMENTS}

I would like to thank Dr. James Carson whose patience and guidance helped me throughout my doctoral degree and who impacted the scientist I am today. I would like to thank my dissertation committee members Dr. Stephen Alway, Dr. Jeremy Loenneke, Dr. Junaith Mohamed, and Dr. Melissa Puppa who have given their time and expertise. I would like to thank the members both past and present of the IMB lab who have assisted me throughout my time: Justin Hardee, Brandon VanderVeen, Dennis Fix, and Jessica Halle and any not specifically mentioned here.

Funding for my dissertation was provided from the National Cancer Institute RO1-CA121249 awarded to Dr. James Carson and from the American College of Sports Medicine Doctoral Award 10-00890 to Brittany Franch. 


\begin{abstract}
Cancer cachexia is defined as the unintentional loss of skeletal muscle mass with or without fat loss that cannot be reversed by conventional nutritional support. Cachexia occurs in $\sim 20 \%$ of cancer patients. More specifically, $50 \%$ of lung cancer patients, the most common cancer worldwide, develop cachexia. Cachexia occurs most often in lung and gastrointestinal cancers, whereas breast and prostate have the lowest rate of cachexia. Cancer-induced cachexia disrupts skeletal muscle protein turnover (decreasing protein synthesis and increasing protein degradation). Skeletal muscle's capacity for protein synthesis is highly sensitive to local and systemic stimuli that are controlled by mTORC1 and AMPK signaling. During cachexia, altered protein turnover is thought to occur through suppressed anabolic signaling via mTORC1, coinciding with the chronic activation of AMPK. While progress has been made in understanding some of the mechanisms underlying the suppressed anabolic signaling in cachectic muscle, gaps still remain in our understanding of muscle's ability to respond to anabolic stimulus prior to cachexia development. The purpose of this study was to determine if cachexia progression disrupts the feeding regulation of AMPK signaling and if gp130 signaling and muscle contraction could regulate this process.
\end{abstract}

Specific aim 1 examined the feeding regulation of skeletal muscle protein synthesis in pre-cachectic tumor-bearing mice. Feeding increased muscle protein synthesis, while lowering AMPK signaling in pre-cachectic tumor-bearing mice. Importantly, pre-cachectic tumor-9bearing mice have overall suppressed muscle protein synthesis independent of the fast or fed condition. Muscle-specific AMPK loss was sufficient to improve the fasting suppression of muscle mTORC1 and protein synthesis in pre-cachectic tumor-bearing mice. Specific aim 2 examined if muscle gp130 signaling regulates the feeding regulation of AMPK during cancer cachexia progression. Muscle gp130 loss lowered the fasting induction of AMPK in pre-cachectic tumor-bearing mice without improving protein synthesis. Muscle gp130 loss did not alter the feeding regulation of muscle Akt/mTORC1 signaling and protein synthesis. Specific Aim 3 examined if an acute bout of muscle contractions could improve the muscle protein synthesis response to feeding during the progression of cachexia. Pre-cachectic tumorbearing mice exhibit suppressed protein synthesis in response low frequency electrical stimulation, and the inability to synergistically induce protein synthesis in response to feeding and contraction.

In summary, pre-cachectic tumor-bearing mice have lowered Akt/mTORC1 signaling and protein synthesis. Feeding can induce Akt/mTORC1 and protein synthesis and AMPK regulates the fasting suppression of protein synthesis in pre-cachectic tumorbearing mice. While gp130 loss reduces AMPK it is not sufficient to improve protein synthesis in pre-cachectic tumor-bearing mice. The added protein synthesis response to feeding and contraction is blunted in pre-cachectic tumor-bearing mice. These findings provide novel insight into the regulation of Akt/mTORC1 signaling and protein synthesis in response to feeding. Additionally, these studies highlight gp130's regulation of AMPK prior to cachexia development, and the blunted anabolic muscle response to feeding and 
contraction in pre-cachectic tumor-bearing mice. By understanding these intracellular signaling processes and perturbations prior to cachexia development, we will be able to elucidate potential therapeutic targets and treatment options to manipulate and prevent cancer cachexia. 


\section{TABLE OF CONTENTS}

CHAPTER 1. INTRODUCTION ...................................................................................

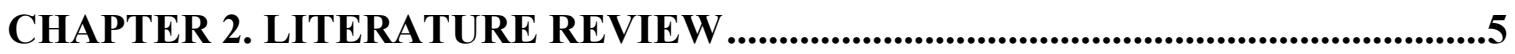

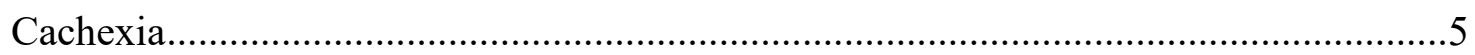

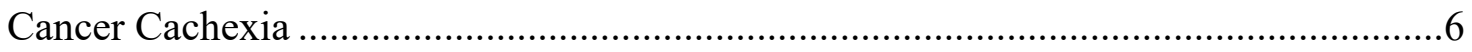

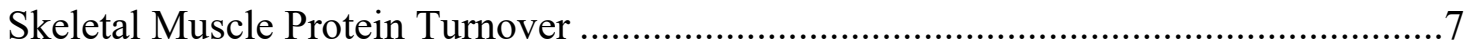

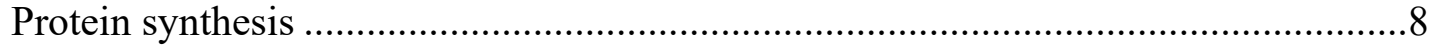

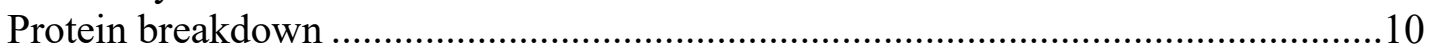

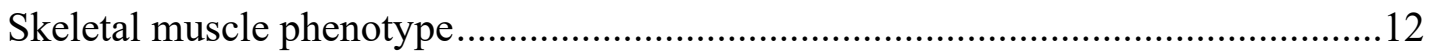

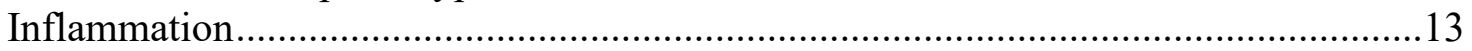

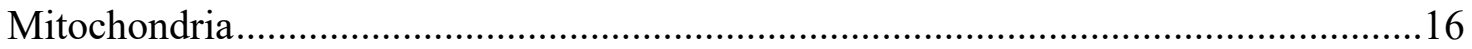

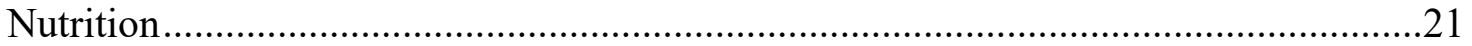

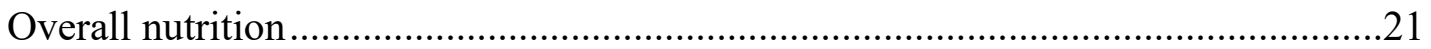

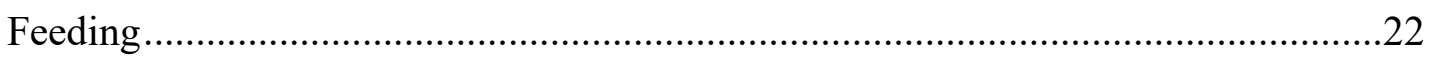

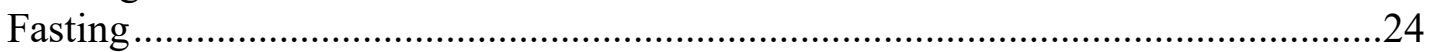

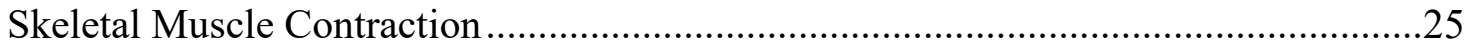

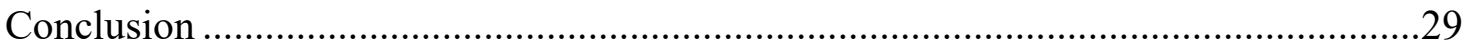

\section{CHAPTER 3. EXAMINATION OF WHETHER SKELETAL MUSCLE AMPK \\ REGULATION BY FEEDING DISRUPTED EARLY IN CANCER CACHEXIA PROGRESSION IS RELATED TO EARLY DISRUPTIONS IN

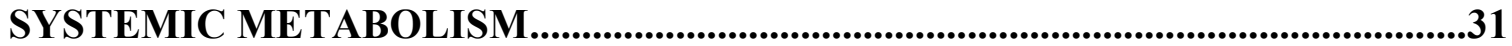

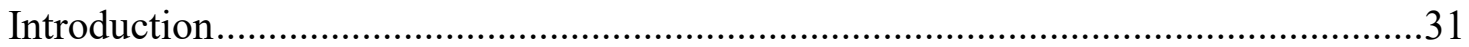

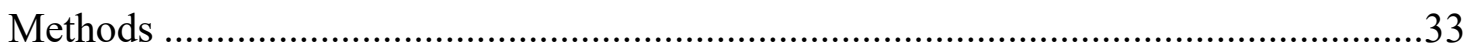

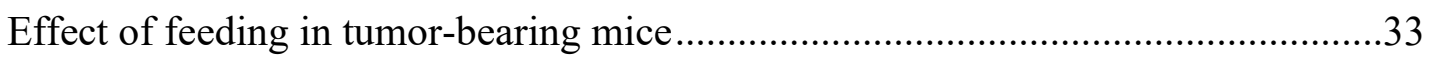

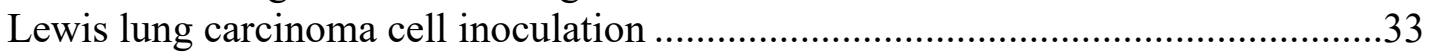

Muscle-specific AMPK loss in tumor-bearing mice.................................................34

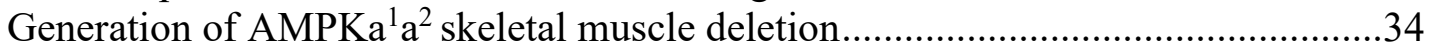

Lewis lung carcinoma cell inoculation in muscle-specific AMPK mice......................34

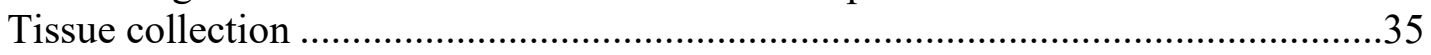

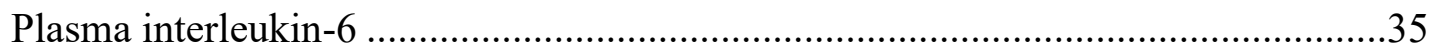

Blood glucose and plasma insulin.......................................................................35

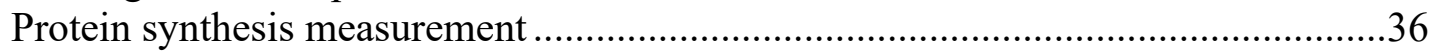

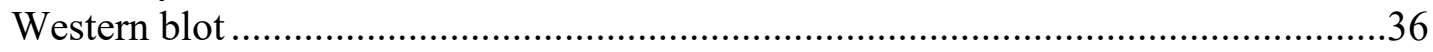

Body composition and indirect calorimetry …………….....................................36

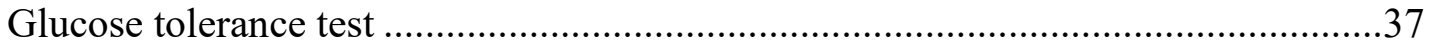

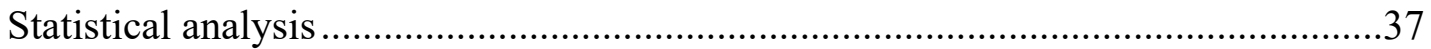

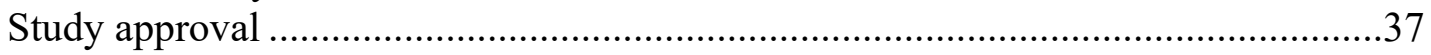

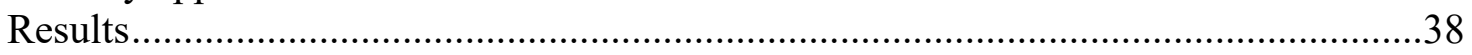

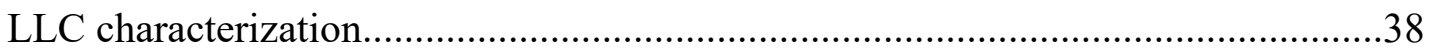

Whole-body response to feeding during cancer cachexia progression .......................42

Muscle Akt/mTORC1 and protein synthesis association to cachexia progression....42

Effect of feeding on AMPK signaling ....................................................................45 
Early onset whole body metabolism and physical inactivity in tumor-bearing mice

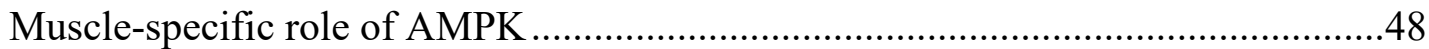

Effect of AMPK loss on skeletal muscle signaling in non-tumor-bearing mice .......50

Validation of muscle-specific AMPK loss in LLC tumor-bearing mice ....................52

Muscle-specific AMPK loss in LLC tumor-bearing male mice does not alter tumor growth, tumor mass, or indices of cachexia .............................................52

Effect of AMPK loss on Akt/mTORC1 signaling and protein synthesis in LLC

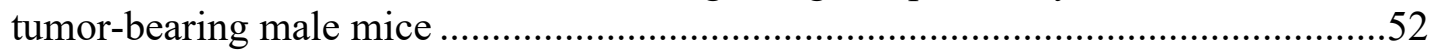

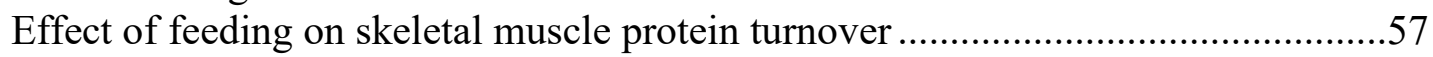

Effect of muscle-specific AMPK loss on skeletal muscle's feeding regulation of

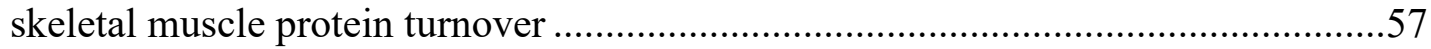

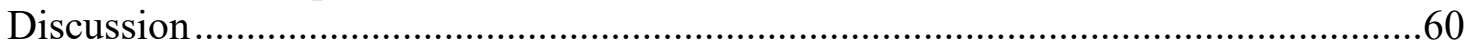

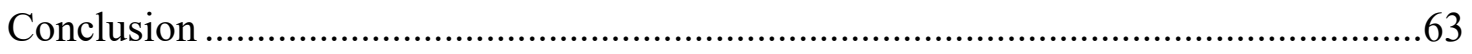

\section{CHAPTER 4. EXAMINATION OF WHETHER MUSCLE GP130 SIGNALING AFFECTS THE FEEDING REGULATION OF AMPK DURING CANCER}

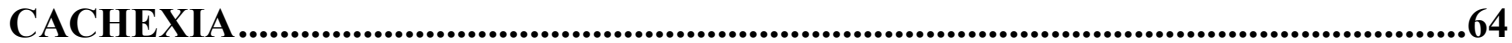

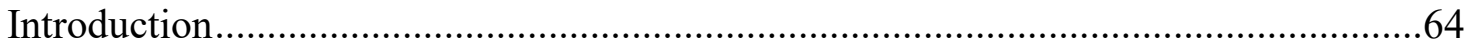

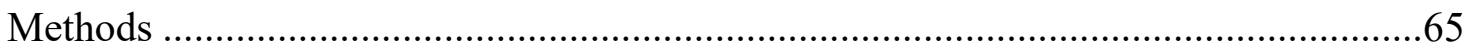

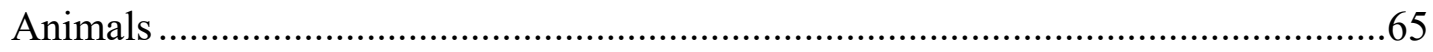

Generation of gp130 skeletal muscle deletion ..................................................66

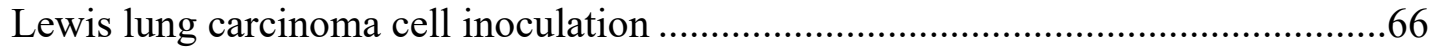

Cell culture experiments ............................................................................66

C2C12 myotube differentiation and conditioned media collection .........................68

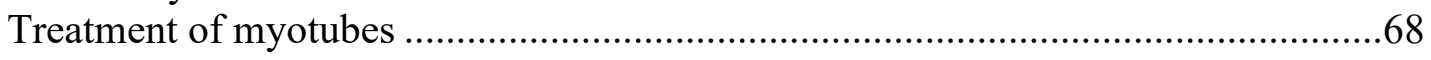

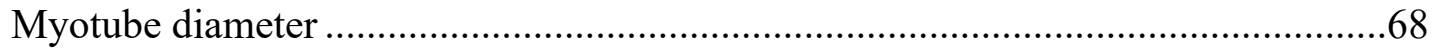

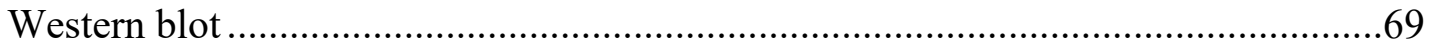

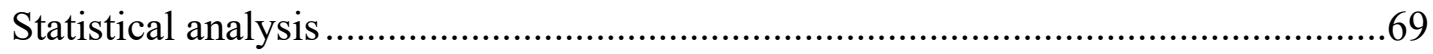

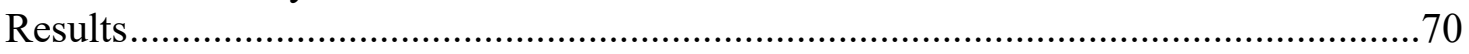

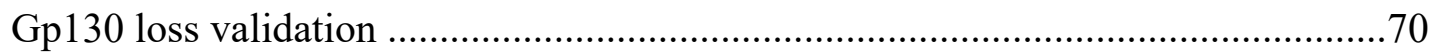

Effect of gp130 loss on skeletal muscle signaling in non-tumor-bearing mice .........70

Validation of muscle-specific gp130 loss in LLC tumor-bearing mice......................72

Muscle-specific gp130 loss in LLC tumor-bearing male mice does not alter tumor growth, tumor mass, or indices of cachexia ............................................. 72

Effect of gp130 loss on AMPK/Akt/mTORC1 signaling and protein synthesis in

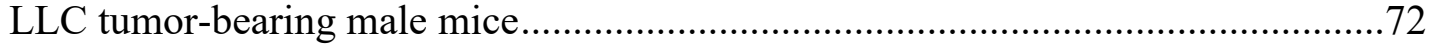
Effect of muscle-specific gp130 loss on skeletal muscle's feeding regulation of skeletal muscle protein turnover .......................................................................... 78 Effect of LLC-conditioned media on Akt/mRORC1 response to insulin in

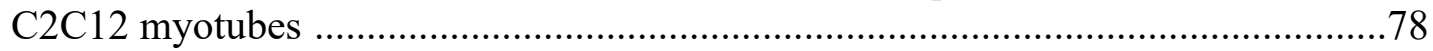

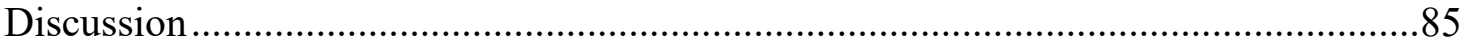

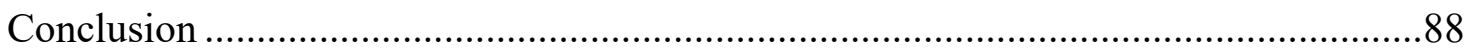




\section{CHAPTER 5. EXAMINATION OF WHETHER AN ACUTE BOUT OF MUSCLE CONTRACTION CAN IMPROVE MUSCLE PROTEIN TURNOVER RESPONSE TO FEEDING DURING THE PROGRESSION OF}

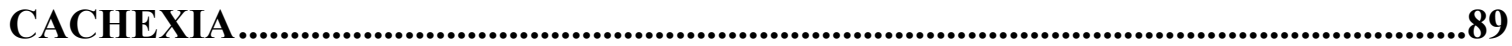

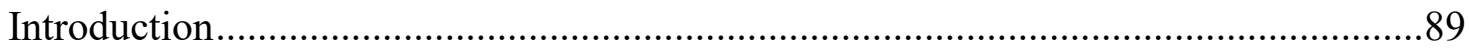

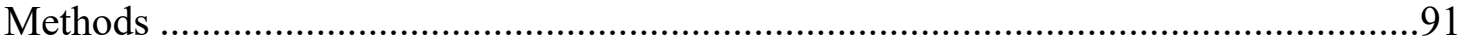

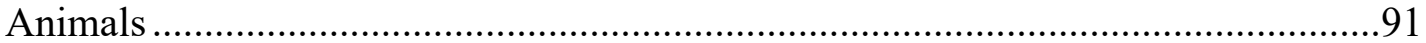

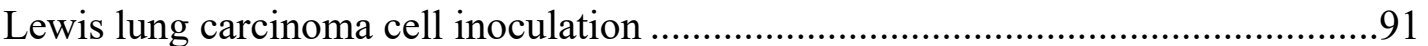

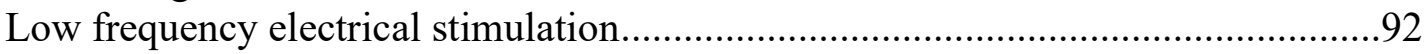

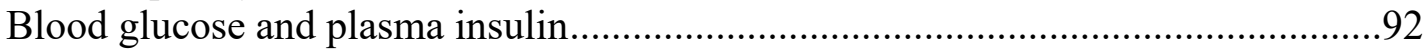

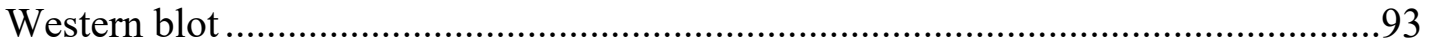

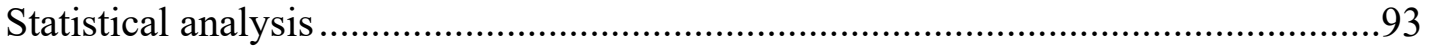

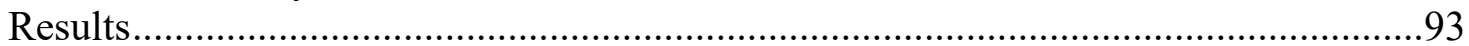

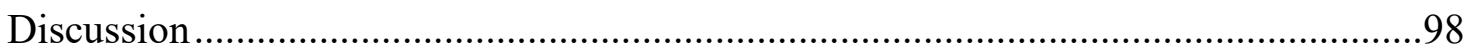

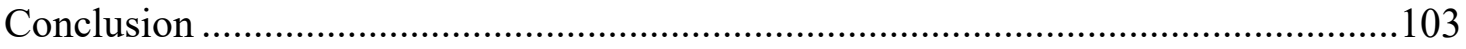

CHAPTER 6. DISCUSSION AND FUTURE DIRECTIONS ...............................104

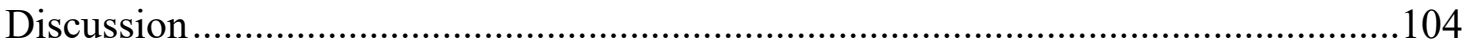

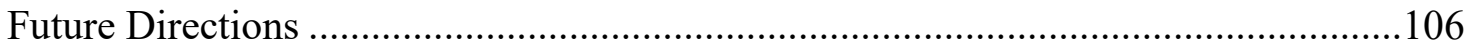

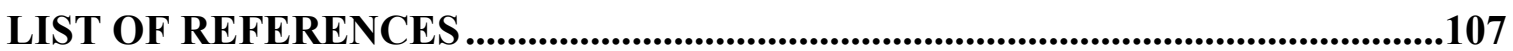

APPENDIX A. ANTIBODY SPECIFICS .........................................................134

APPENDIX B. FEEDING AND FASTING SUPPLEMENTAL DATA ..................135

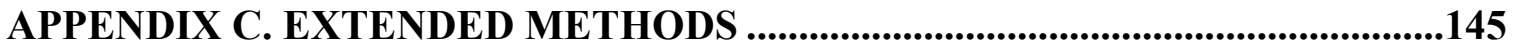

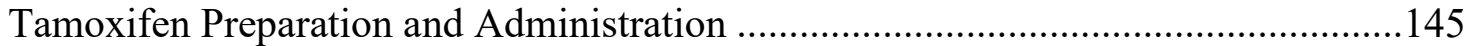

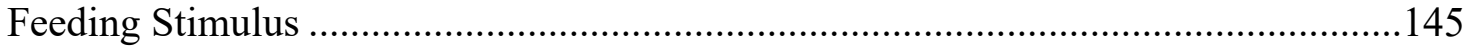

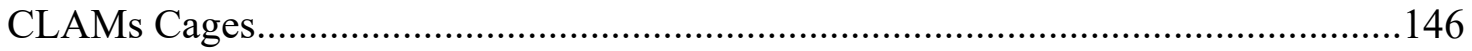

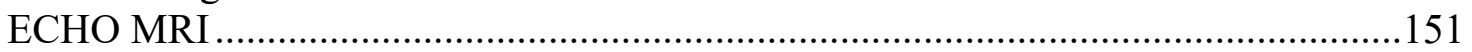

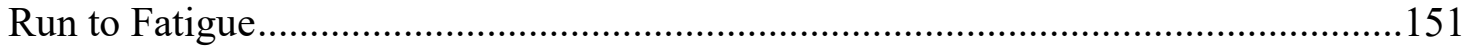

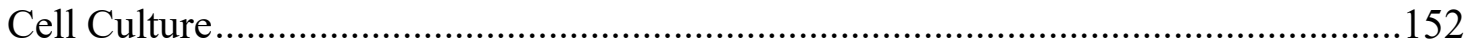

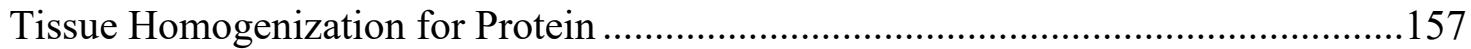

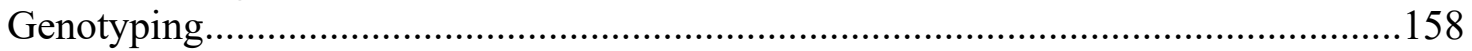

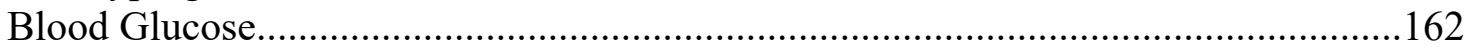

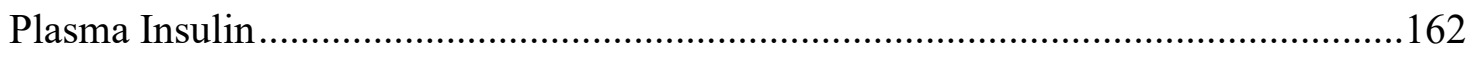

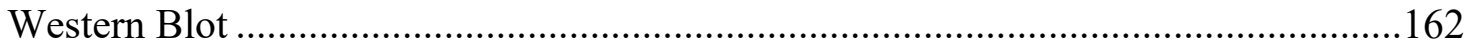

VITA 


\section{LIST OF TABLES}

Table 3-1. Animal characteristics of LLC tumor-bearing male mice

Table 3-2. Animal characteristics of PBS and LLC male mice in the fast and fed condition

Table 3-3. Whole body response to feeding in PBS and LLC male mice

Table 3-4. Muscle-specific AMPK loss does not alter animal characteristics in PBS treated male mice

Table 3-5. Effect of muscle-specific AMPK loss on animal characteristics in LLC tumor-bearing mice ........................................................................55

Table 3-6. Whole-body response to feeding in PBS and LLC male mice .....................58

Table 4-1. Muscle-specific gp130 loss on non-tumor-bearing mice animal characteristics

Table 4-2. Effect of muscle-specific gp130 loss on animal characteristics in LLC

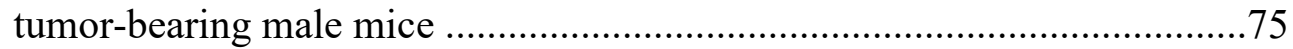

Table 4-3. Whole-body response to feeding in PBS and LLC male mice ....................79

Table 5-1. Animal characteristics of PBS and LLC mice in the fast and fed condition $3 \mathrm{hrs}$ post LFES

Table 5-2. Whole-body response to feeding in PBS and LLC male mice .96

Table A-1. Antibody specifics 134

Table B-1. Associations of early onset systemic metabolism and physical activity to indices ot cachexia and tumor development

Table B-2. Associations of early onset systemic metabolism and physical activity to AMPK association signaling in fast and fed LLC mice 


\section{LIST OF FIGURES}

Figure 1-1. Working model

Figure 3-1. LLC tumor-bearing male mice exhibit several indices of cachexia and increased skeletal muscle inflammatory signaling....

Figure 3-2. LLC tumor-bearing mice exhibit whole-body and muscle-specific anabolic response to 1-hr ad libitum feeding

Figure 3-3. 1-hr of ad libitum feeding is sufficient to lower skeletal muscle AMPK in LLC tumor-bearing mice

Figure 3-4. Increased AMPK signaling by fasting is associated to fat wasting in LLC tumor-bearing mice

Figure 3-5. Muscle-specific AMPK loss in male PBS mice

Figure 3-6. Increased AMPK signaling can be reduced by muscle-specific AMPK loss in LLC tumor-bearing mice

Figure 3-7. Muscle-specific AMPK loss in LLC tumor-bearing mice does not alter tumor mass, tumor growth, or indices of cachexia

Figure 3-8. Muscle-specific AMPK loss is sufficient to increase muscle protein synthesis in LLC tumor-bearing mice following a $12 \mathrm{hr}$ fast

Figure 3-9. Effect of muscle-specific AMPK loss on skeletal muscle's feeding regulation of $\mathrm{Akt} / \mathrm{mTORC} 1$ signaling and protein synthesis .....

Figure 4-1. Muscle-specific loss of gp130 in male mice

Figure 4-2. Validation of muscle gp130 knockdown and associated downstream signaling in LLC tumor-bearing male mice

Figure 4-3. Muscle-specific loss of gp130 in LLC tumor-bearing male mice does not alter tumor growth, tumor mass, or indices of cachexia

Figure 4-4. Muscle-specific gp130 loss reduces AMPK in LLC tumor-bearing mice....76

Figure 4-5. Muscle-specific gp130 loss does not rescue suppressed protein synthesis in LLC tumor-bearing mice......

Figure 4-6. Muscle-specific gp130 loss disrupts AMPK's reduction by feeding.....

Figure 4-7. Muscle-specific gp130 loss regulation of Akt/mTORC1 signaling and protein synthesis response to feeding in LLC tumor-bearing mice 
Figure 4-8. LLC conditioned media suppresses myotube growth and induces

inflammatory associated signaling in $\mathrm{C} 2 \mathrm{C} 12$ myotubes

Figure 4-9. LLC conditioned media suppresses the insulin activation of Akt and mTORC1 signaling in C2C12 myotubes

Figure 4-10. IL-6 nor lif were sufficient to suppress Akt/mTORC1 induction by insulin in $\mathrm{C} 2 \mathrm{C} 12$ myotubes

Figure 5-1. LLC tumor-bearing mice exhibit a blunted protein synthesis response to LFES......

Figure 5-2. LFES and feeding exert synergistic effect on skeletal muscle protein synthesis in non-tumor-bearing mice

Figure 5-3. LLC inhibits the synergistic effect of LFES and feeding on skeletal muscle protein synthesis in tumor-bearing mice.

Figure 5-4. LLC tumor-bearing mice have altered feeding and contraction regulation of AMPK and STAT3

Figure B-1. The effect of LLC on skeletal muscle signaling in male mice following an overnight $12 \mathrm{hr}$ fast.

Figure B-2. The effect of feeding in PBS male mice following an overnight $12 \mathrm{hr}$ fast 136

Figure B-3. Early onset whole body metabolism and physical activity in a subset of LLC tumor baring mice

Figure B-4. The effect of muscle-specific AMPK loss in PBS male mice following an overnight $12 \mathrm{hr}$ fast

Figure B-5. The effect of muscle-specific AMPK loss on the feeding regulation of muscle protein turnover in PBS male mice.

Figure B-6. The effect of muscle-specific AMPK loss on the feeding regulation of muscle protein turnover in LLC male mice

Figure B-7. The effect of muscle-specific gp130 loss on skeletal muscle protein turnover in PBS male mice following an overnight $12 \mathrm{hr}$ fast.

Figure B-8. The effect of muscle-specific gp130 loss on skeletal muscle inflammatory signaling and mitochondrial quality control in PBS and LLC male mice following $1 \mathrm{hr}$ ad libitum feeding. 


\section{LIST OF ABBREVIATIONS}

ACC

ADP

AIDS

Akt

AMPK

$A p c^{\mathrm{Min} /+}$

ATG

ATP

Atrogin-1/ MAFbx

$\mathrm{Balb} / \mathrm{c}$

BAX

C26

C57BL/6

$\mathrm{Ca} 2+$

CaMKK2

CNTF

$\mathrm{COX}$

CPT-1

DEPDC5

DMSO

DRP1

EDL

eIF

ERK1/2

ES-2

Fis 1

FOXO

Gator $1 / 2$

GLUT

gp130

Grb2

GSK-3

HFES

HT-29

IGF-1

IL-6

JAK

JNK

$\mathrm{KPC}$

LC3B

LFES

IF

LKB1

LLC
Acetyl-CoA carboxylase

Adenosine diphosphate

Acquired immunodeficiency syndrome

Protein kinase B (PKB)

5' AMP-activated protein kinase

Adenomatous polyposis coli Min/+

Autophagy related

Adenosine triphosphate

Muscle atrophy F box

Bagg albino laboratory bred/ genotype c

Pro-apoptotic protein

Colonic Adenocarcinoma 26

C57 black 6

Calcium

Calcium/calmodulin-dependent kinase kinase 2

Ciliary neutrophilic factor

Cytochrome C oxidase

Carnitine palmityltransferase-1

DEP domain containing 5

Dimethyl sulfoxide

Dynamin related protein 1

Extensor digitorum longus

Eukaryotic translation initiation factor

Extracellular signal-regulated kinase 1 and 2

Homo sapiens ovary clear cell carcinoma

Fission 1 protein

Forkhead box transcription factors

GTPase-activating protein for Rag

Glucose transporter type

Glycoprotein-130

Growth-factor receptor bound protein 2

Glycogen synthase kinase 3

High frequency electrical stimulation

Human colorectal adenocarcinoma-29

Insulin-like growth factor 1

Interleukin 6

Janus kinase

c-Jun N-terminal kinases

Kras, P53, and Cre

Microtubule-associated protein 1A/1B-light chain

Low frequency electrical stimulation

Leukemia inhibitory factor

Liver kinase B1

Lewis lung carcinoma 


\begin{tabular}{|c|c|}
\hline MAPK & Mitogen-activated protein kinases \\
\hline MCU & Mitochondrial calcium uniporter \\
\hline MEF2 & Myocyte enhancer factor 2 \\
\hline MFN & Mitofusion \\
\hline $\mathrm{MHC}$ & Myosin heavy chain \\
\hline mTOR & Mechanistic target of rapamycin \\
\hline mTORC1 & Mechanistic target of rapamycin complex 1 \\
\hline mTORC2 & Mechanistic target of rapamycin complex 2 \\
\hline MuRF1 & Muscle ring finger 1 \\
\hline NAD & Nicotinamide adenine dinucleotide \\
\hline $\mathrm{NF}-\kappa \mathrm{B}$ & Nuclear factor kappa-light-chain-enhancer of activated B cells \\
\hline NPRL $2 / 3$ & Nitrogen permease regulator-like 2 \\
\hline NSCLC & Non-small cell lung cancer \\
\hline OPA1 & Optic atrophy protein 1 \\
\hline OSM & Oncostatin M \\
\hline P53/TP53 & Tumor protein \\
\hline P62/SQSTM1 & Sequestosome- \\
\hline p70S6K & Ribosomal protein S6 kinase beta 1 \\
\hline PBS & Phosphate buffered saline \\
\hline PDK1 & Phosphoinositide-dependent kinase-1 \\
\hline PDTC & Pyrrolidine dithiocarbonate \\
\hline PGC-1 $1 \alpha$ & Peroxisome proliferator-activated receptor gamma coactivator-1 $\alpha$ \\
\hline PI3K & Phosphatidylinositol-4,5-bisphosphate 3-kinase \\
\hline Rheb & Ras's homolog enriched in brain \\
\hline RPS6 & Ribosomal protein S6 \\
\hline SHP-2 & Src homology domain-containing protein tyrosine phosphatase \\
\hline SOCS3 & Suppressor of cytokine signaling 3 \\
\hline STAT3 & Signal transducer and activator of transcription 3 \\
\hline TA & Tibias anterior \\
\hline TCB1D1 & Tab-GTPase-activating protein \\
\hline Tfam & Transcription factor A \\
\hline TFEB & Transcription factor EB \\
\hline TNF- $\alpha$ & Tumor necrosis factor $\alpha$ \\
\hline TOV21G & Homo sapiens ovary cell carcinoma grade 3 \\
\hline $\mathrm{TSC} 1 / 2$ & Tuberous sclerosis complex protein 1 and 2 \\
\hline ULK & Unc-51 like autophagy activating kinase \\
\hline UPS & Ubiquitin proteasome system \\
\hline 4EBP1 & Eukaryotic translation initiation factor 4E-binding protein 1 \\
\hline
\end{tabular}




\section{CHAPTER 1. INTRODUCTION}

Cancer cachexia is defined as the unintentional loss of muscle mass with or without fat loss, and is irreversible with nutritional support (Evans et al., 2008). Cancerassociated cachexia occurs in $\sim 20 \%$ of cancer patients and is the cause of $40 \%$ of all cancer related deaths (Evans et al., 2008). Lung cancer is the most common cancer worldwide, with $50 \%$ of lung cancer patients developing cachexia (Jafri et al., 2015). The overall 5-year survival rate for lung cancer patients is $19 \%$, however this is greatly impacted by cancer stage (Siegel et al., 2020). However, the survival rate falls to only 5\% for patients diagnosed at distant stages (Siegel et al., 2020). Therefore, therapeutic strategies to prevent or minimize cancer induced cachexia are needed to improve patient survival and life quality.

Skeletal muscle's capacity for protein synthesis and degradation is highly sensitive to local and systemic stimuli that are integrated intracellularly through mTORC1 and AMPK signaling (Frost \& Lang, 2007). Oscillations in protein synthesis and breakdown occur throughout the day in response to feeding and fasting (Breen \& Phillips, 2011). Briefly, feeding elicits an increase in circulating glucose to promote insulin release by the pancreas thereby translocating GLUT4 to the muscle's membrane to remove circulating glucose (Richter \& Hargreaves, 2013) and circulating insulin can also directly bind to IGF-1 resulting in a confirmational change to induce downstream phosphorylation of IRS/AKT/mTORC1 (Boucher et al., 2014). Feeding can directly target mTORC1 by increasing amino acid pool to promote protein synthesis (Liu \& Sabatini, 2020). Alternatively, fasting in pre-clinical models (24-48hrs) elicits an energy stress demand resulting in increased muscle AMPK signaling (Bujak et al., 2015). AMPK is a regulatory point of interaction due to its numerous effects on muscle protein turnover by lowering ATP consumption of anabolic pathways and increasing catabolic pathways to produce ATP. AMPK is activated by energy stress when ATP bound to AMPK subunit is displaced with AMP, which occurs by reduced ratios of ATP:ADP and increased ratio of AMP:ATP. Throughout the day in healthy tissue, ATP:ADP ratios are in constant flux and exhibit a circadian rhythm with AMPK lowered after the dark cycle (Um et al., 2011). To disrupt these ratios, either there is an interference of ATP production or increased ATP consumption (Hardie, 2003). During periods of fasting, blood glucose levels begin to fall which interferes with ATP production, therefore, glucose availability is increased by stimulating liver glucose production. Furthermore, during longer periods of fasting after glycogen stores have been depleted, metabolism of triglycerides are used through beta oxidation and ketogenesis to produce ATP (Herzig \& Shaw, 2018). While these physiological changes to feeding and fasting occur throughout the day in healthy populations, significant barriers still exist in our understanding of how cachexia progression disrupts mTORC1 and AMPK signaling by feeding.

Cancer-induced cachexia disrupts skeletal muscle protein turnover in the basal condition (decreasing protein synthesis and increasing protein degradation) (Aversa et al., 2016; Baracos, 2000). In pre-clinical models, our laboratory has demonstrated altered protein turnover through the suppression of anabolic signaling via mTORC1 which 
coincided with chronic activation of AMPK (White, Baynes, et al., 2011). Recent work from our lab has shown that mTORC1 signaling exhibits diurnal fluctuations in response to changes throughout the day in feeding and activity (Counts et al., 2020). Interestingly, the cachectic environment disrupts these diurnal fluctuations, and suggest a link between disruptions in feeding and activity behaviors to the progression of cachexia and altered mTORC1 signaling (Counts et al., 2020). While our lab and others have shown that the cachectic environment's chronic activation of aberrant AMPK induces a higher basal state of autophagy signaling (Penna, Ballaro, Martinez-Cristobal, et al., 2019; White, Baynes, et al., 2011), recent unpublished data has highlighted that a short-term fast accelerates protein degradation through AMPK in cachectic muscle. These findings have significant implications because more evidence is identifying a potential therapeutic window based on the time of day (Zhao et al., 2020) and nutritional state of the patient (Lammers et al., 2020). However, significant barriers still exist in our understanding of cachectic skeletal muscle's protein synthesis response by fasting and feeding.

Inflammation has been implicated as a key mediator of cancer-induced cachexia leading to chronic elevation of skeletal muscle STAT3 and MAPK activity (Deans \& Wigmore, 2005). Glycoprotein-130 (gp130) is a regulatory point of interaction between systemic inflammation and JAK/STAT and MAPK muscle signaling (White \& Stephens, 2011). We have previously reported muscle gp130 loss prevented Lewis lung Carcinoma (LLC) induced muscle wasting and restored STAT3 and MAPK signaling (Puppa, Gao, et al., 2014). Interestingly, the LLC model has known disruptions in mitochondrial function prior to muscle wasting (Brown et al., 2017). Additionally, disruptions to the muscle mitochondria are known to induce AMPK, thereby accelerating muscle wasting (VanderVeen et al., 2017). Given that muscle gp130 can regulate mitochondrial quality control (Hardee, Counts, et al., 2018), identifying if these are linked to cachexia disruption of protein turnover by feeding is warranted.

Exercise training is a known inducer of anabolic signaling through mTORC1 (Burd et al., 2009) and reduces chronic systemic inflammation (Beavers et al., 2010). An acute bout of skeletal muscle contraction is sufficient to induce glucose uptake, fatty acid oxidation, mitochondrial biogenesis, and protein synthesis (Egan \& Zierath, 2013). Whereas repeated exercise bouts can induce functional adaptations in contractile proteins, mitochondrial function, and metabolic regulation (Egan \& Zierath, 2013). Exercise has been hypothesized to be a potential therapeutic option for cancer induced cachexia (Hardee et al., 2019). We have recently shown that repeated eccentric contractions are sufficient to induce anabolic signaling in the cachectic environment, coinciding with suppressed inflammatory signaling in glycolytic muscle (Hardee et al., 2020). Additionally, we highlight repeated contractions improved muscle oxidative capacity further supporting that cachectic muscle has the plasticity to respond to contraction stimulus. While high frequency eccentric contractions show promising results in preclinical models, clinical populations hesitate to employ such a potent stimulus therefore rehabilitation settings utilize a low-to-moderate frequency of contraction/load (Hody et al., 2019). We have previously shown that an acute bout of low-frequency electrical stimulation (LFES) induced downstream mTORC1 signaling and oxidative capacity, however this response was suppressed in cachectic muscle (Puppa, Murphy, et 
al., 2014). While we have made advances in our understanding of cachectic muscle's response to acute contraction, significant barriers still exist in understanding if the suppressed contraction response is greater in more severe cachexia.

The anabolic response of resistance exercise in combination with nutritional stimulus have been highly sought after in all populations (Barclay et al., 2019; Breen \& Phillips, 2013; Moore, 2019; Symons et al., 2011; Trommelen et al., 2019); specifically, in the elderly or during disease in hopes to offset anabolic resistance. Several studies have investigated skeletal muscle's response to contraction and/or mechanical signaling (Hornberger, 2011), however very little work has been done to examine the combined effects of contraction and feeding on skeletal anabolic signaling (Gordon et al., 2014; Nakayama et al., 2019) specifically during disease (Hardee, Counts, et al., 2018; Puppa, Murphy, et al., 2014; Sato et al., 2019) and disuse (Roberson et al., 2020; Shimkus et al., 2018). Interestingly, there is promising work that the combined effects of exercise and nutrient supplementation can increase muscle protein synthesis to a greater extent than feeding alone in prostate cancer patients (Hanson et al., 2017). There are gaps in our understanding of how cancer cachexia disrupts the muscle metabolic response to feeding and whether contraction can alter these responses. The purpose of the current project was to determine if cachexia progression disrupts the feeding regulation of AMPK signaling and if gp130 signaling and muscle contraction could regulate this process; see working model (Figure 1-1). Our central hypothesis was that the feeding regulation of muscle AMPK was disrupted at cancer cachexia initiation. Furthermore, the dysregulation of AMPK signaling was caused by muscle gp 130 signaling, and an acute bout of muscle contractions could improve the feeding regulation during cancer cachexia progression.

SPECIFIC AIM 1: To determine if skeletal muscle AMPK regulation by feeding was disrupted early in cancer cachexia progression and if this was related to early disruptions in systemic metabolism.

SPECIFIC AIM 2: To examine if muscle gp130 signaling regulates the feeding regulation of AMPK during cancer cachexia.

SPECIFIC AIM 3: To examine if an acute bout of muscle contraction could improve the muscle protein synthesis response to feeding during the progression of cachexia. 


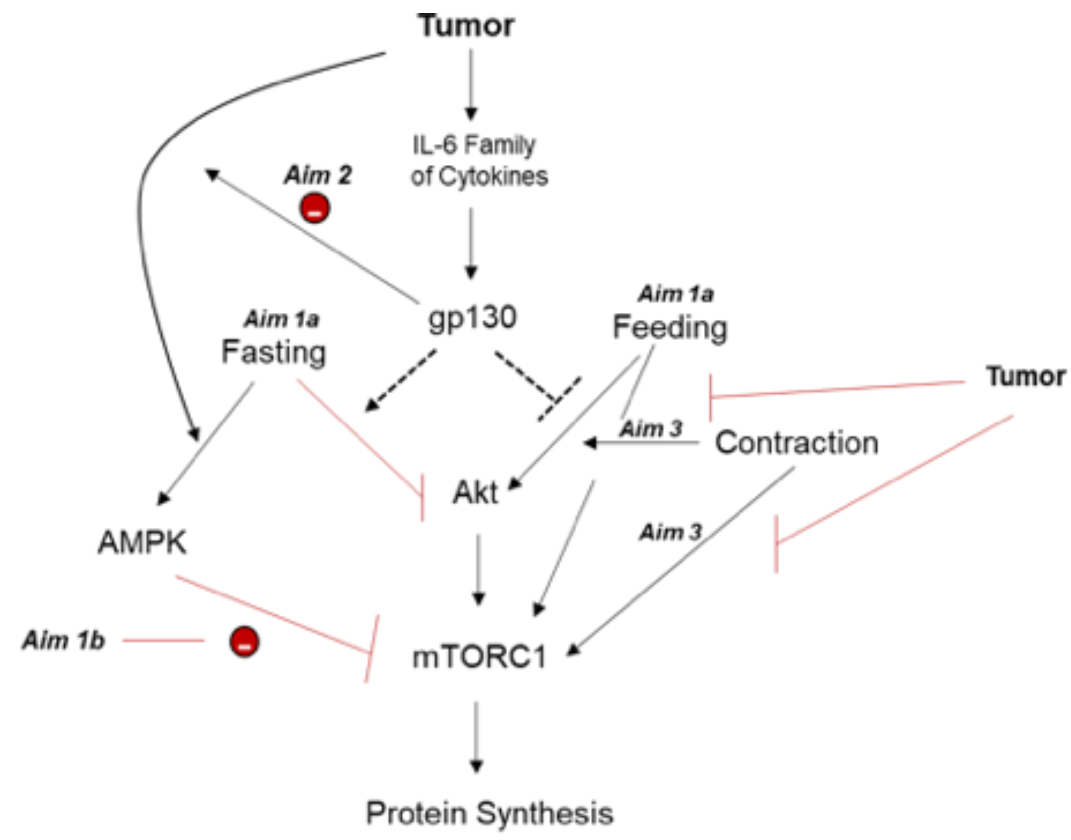

Figure 1-1. Working model 


\section{CHAPTER 2. LITERATURE REVIEW}

\section{Cachexia}

Cachexia is a severely debilitating condition that accompanies many chronic diseases such as AIDs, renal failure, heart disease, and cancer. Cachexia is defined as the unintentional loss of muscle mass with or without fat loss and is irreversible with nutritional support (Evans et al., 2008; Muscaritoli et al., 2010). Cancer-associated cachexia occurs in $\sim 20 \%$ of cancer patients(Evans et al., 2008). Cachexia progression is predominantly characterized by body weight loss over time and has been positively associated to reduced survival (Evans et al., 2008; von Haehling et al., 2009).

Furthermore, the most recent consensus on cancer associated cachexia is defined as three phases, and each phase's progression is determined by the cancer type, stage, systemic inflammation, inactivity, treatment, and food intake (Fearon et al., 2012; Roeland et al., 2020). Additionally, pre-clinical studies and clinical trials have greatly improved our understanding of the condition, however we still lack sufficient evidence to provide therapeutic treatments to reverse to prevent cancer-induced cachexia.

Lung cancer is the most common cancer worldwide, with $50 \%$ of lung cancer patients developing cachexia (Jafri et al., 2015). The overall 5-year survival rate for lung cancer patients is $19 \%$, however this is greatly impacted by the stage of diagnosis, for example local or regional non-small cell lung cancer (NSCLC) is relativity high (31$57 \%$ ) (Siegel et al., 2020). However, the survival rate falls to only $5 \%$ for patients diagnosed at distant stages (Siegel et al., 2020). The significant reduction in survival rate is associated with a late-stage diagnosis compounded by toxic therapeutics. For example, $\sim 60 \%$ of lung cancer patients reported weight loss prior receiving treatment, which was associated with reduced survival and increased chemotherapy toxicity (Ross et al., 2004). Due to the complexity of the condition, understanding the mechanisms involving lung cancer development and cancer-induced systemic dysfunction that promote cachexia are needed to improve patient survival.

Cachexia is not present in all cancer types, however those patients that develop cachexia are more susceptible to chemotherapy toxicity, reduced recovery time after surgery, increased infection rate, and decreased survival (Baracos et al., 2018; Esper \& Harb, 2005; von Haehling et al., 2009). The cachectic condition exhibits increased systemic inflammation, altered metabolism, hypogonadism, and overall weakness (Baracos et al., 2018; Tisdale, 2010), likely contributing to the abovementioned adverse outcomes. Recent advances in understanding cachexia development have highlighted the importance of muscle mass maintenance (Baracos et al., 2019). Importantly, skeletal muscle is a strong predictor of survival in cachectic patients (Cho et al., 2017). For example, in cachectic patients and pre-clinical models, diaphragm muscle mass loss during cachexia lead to decreased work capacity, reduced respiration function, and increased morbidity (Greising et al., 2018; Roberts, Ahn, et al., 2013). In addition, cachectic patients exhibit decreased physical function (Fearon et al., 2006), which was associated to greater body weight loss (Fouladiun et al., 2007). Lastly, pre-clinical 
models have highlighted that physical function decrements precede severe wasting suggesting there might be a point of intervention to stave off cachexia (Counts et al., 2020; VanderVeen et al., 2018). Taken together, these studies highlight the importance of muscle mass health during cancer cachexia progression, however the complexity of the condition has provided difficulty in understanding the molecular mechanisms that promote cancer associated cachexia are poorly understood.

\section{Cancer Cachexia}

Understanding and determining the underlying mechanisms of cancer induced cachexia have been long sought after. Approximately $40 \%$ of cancer patients will experience progressive wasting of adipose or skeletal muscle tissue (Evans et al., 2008). Human studies have been used to explore the mechanisms of cachexia progression, however, there are currently limited studies that have defined the cachectic response in cancer patients. This is in part due to the difficulty related to obtaining patient samples, inability to control for cancer stage, tumor type, and therapeutics, thus pre-clinical models are commonly used to eliminate confounders (Baracos, 2018). Interestingly, recent work has sought to determine the most translatable pre-clinical model that exhibit similar systemic features of human patients (Talbert et al., 2019), however this is only in the beginning stages and is limited to pancreatic cancer. Our techniques of modeling human cancer cachexia will advance, to date there are several established murine models to examine the initiation and progression of cancer cachexia that us to elucidate the underlying mechanisms of wasting (Ballaro et al., 2016). While it is acknowledged that many different pre-clinical models are available, the following section will highlight mouse and rat models that have been commonly used by our laboratory and others and promising models for the examination of cancer induced muscle wasting.

There are many types of pre-clinical models in mice and rats used to examine cancer induced cachexia, however there are several more mouse models. First, one widely used mouse model of cancer induced cachexia is the $A p c^{\mathrm{Min} /+}$ which spontaneously develops intestinal polyps. The mouse has a mutation in the Adenomatous polyposis coli gene (Apc) which predispositions the mouse for multiple intestinal adenomas (Min) (Moser et al., 1990). The Apc ${ }^{\mathrm{Min} /+}$ model is commonly used because of the slower development of cachexia compared to tumor implantation models.

Additionally, this model's cachexia progression is closely related to tumor burden and circulating IL-6 levels (Baltgalvis et al., 2010; White et al., 2012). The C26 adenocarcinoma model of cancer cachexia is an implantable tumor model in Babl/c mice. The C26 model will develop one large non-metastatic tumor, and cachexia progression is associated to tumor mass and plasma IL-6 (Bonetto et al., 2012). Cachexia progression in the C26 model occurs rapidly, often within 2-3 weeks thus limiting the therapeutic window. Most studies utilizing the $\mathrm{C} 26$ model investigate interventions to prevent cachexia. The murine adenocarcinoma 16 (Mac 16) model of cancer induced cachexia is used in nude Balb/c mice and suggest being independent of circulating cytokines (Monitto et al., 2001). The Mac 16 model of cancer induced cachexia is known to elicit 
anorexia, however late-stage glucose and insulin signaling were independent of food consumption.

Similar to C26, the implantable Lewis lung carcinoma (LLC) is another common model of cancer induced cachexia. There are several murine models used to evaluate lung cancer progression and treatment options to improve survival, however very few develop cachexia (Kellar et al., 2015). The LLC model is a syngeneic xenograph model used to study lung cancer in the C57Bl/6 mouse. The LLC model is commonly used due to its tumorigenic properties, responsiveness to chemotherapy, and ability to metastasize to the lungs and liver (Kellar et al., 2015). While there are a few other preclinical models to examine lung cancer, these models are implemented in either Balb/c or nude mice, and not all of them have been shown to exhibit cachexia (Kellar et al., 2015). Furthermore, utilizing the LLC model in $\mathrm{C} 57 \mathrm{Bl} / 6 \mathrm{~J}$ allows for the use of transgenic animal models. Lastly, the LLC model is commonly used to examine early and late-stage cancer cachexia phenotype by evaluating the animal at least at two different timepoint post tumor inoculation (Chiappalupi et al., 2020; Han et al., 2020; Sun et al., 2016). Additionally, recent studies have identified metabolic dysfunction in LLC mice and was more pronounced in mice with larger tumors (Han et al., 2020), thus, highlighting the heterogeneity within the LLC model (Counts et al., 2021).

Less investigated animal models of cancer cachexia, either by recent advances in technology, translational difficulty or feasibility are the KPC, TOV21G, ES-2, and HT-29 models of cancer induced cachexia. KPC model of cancer cachexia (KRAS and mutant $\mathrm{P} 53$ allele under pancreas specific Pdx-1 cre) is a recent model used to investigate pancreatic ductal adenocarcinoma (PDAC) and development of cachexia can occur as early as 5 days post tumor implantation (Michaelis et al., 2017). The TOV21G cells (homo sapiens ovary cell carcinoma grade 3 ) induced muscle wasting and exhibited elevated IL-6, and increased autophagy flux suggesting an IL-6/autophagy driven muscle wasting model (Pettersen et al., 2017). Additionally, another very recent model of cancer induced cachexia utilized ES-2 cells (Homo sapiens ovary clear cell carcinoma) in immunodeficient mice and has only been minimally investigated (Pin et al., 2018). Lastly, the HT-29 model of cancer induced cachexia is a human colorectal adenocarcinoma cell line utilized in nude mice (Bonetto, Kays, et al., 2016). While we have made advances in developing new models of cancer induced cachexia and aid in targeting the heterogeneity of the condition, nevertheless, is remains ever important to consider the mechanism of interest and efficacy of the model prior to study development.

\section{Skeletal Muscle Protein Turnover}

Skeletal muscle comprises $40 \%$ of the bodies total weight and has regulation of whole-body metabolism (Zurlo et al., 1990). Skeletal muscle is necessary for body posture and daily movement. Loss of skeletal muscle is a hallmark of cancer cachexia, and muscle mass loss is directly related to increased morbidity and mortality. Given that the maintenance of skeletal muscle mass and metabolic function are critical for health (Wolfe, 2006), understanding the regulation of muscle wasting is critical for the 
development of therapeutic strategies for the proper treatment of cancer cachexia. Skeletal muscle is a highly plastic tissue in which its homeostasis can be influenced by the balance between the rates of protein synthesis and degradation, termed protein turnover (Maddocks et al., 2011; Marimuthu et al., 2011). Given the importance of skeletal muscle to overall health, understanding the mechanisms regulating protein synthesis and breakdown are critically important for improving cancer cachexia treatments. The next few sections will provide understanding into the regulation of skeletal muscle protein turnover and how these processes are altered by cachexia.

\section{Protein synthesis}

Skeletal muscle protein turnover oscillates throughout the day in response to exercise and feeding (Burd et al., 2009; Phillips, 2014). Skeletal muscle protein synthesis is regulated by several factors such as nutrient status, activity level, and inflammation. Protein kinase B (PKB or Akt) and the mechanistic / mammalian target of rapamycin complex 1 (mTORC1) have established roles for the integration of anabolic signaling initiated by growth factors, nutrients, and mechanical loading to regulate protein synthesis (Laplante \& Sabatini, 2009). Insulin and insulin-like growth factor 1 (IGF1) are growth factors that stimulate mTORC1 signaling through the activation of Akt. Binding of insulin/IGF1 to its cell surface receptor initiates phosphoinositide 3-kinase (PI3K)dependent activation of Akt T308 through phosphoinositide-dependent kinase 1 (PDK1). Additionally, rapamycin-insensitive mTOR complex 2 (mTORC2) has also been shown to be regulated by growth factors through the phosphorylation of Akt S473 (Fayard et al., 2005). Furthermore, downstream target of Akt to control protein synthesis are glycogen synthase kinase-3B (GSK3B), proline-rich Akt substrate $40 \mathrm{kDa}$ (PRAS40), and tuberous sclerosis 2 (TSC2) (Fayard et al., 2005). Briefly, GSK3B is inhibited by Akt phosphorylation, which leads to the activation of eukaryotic translation initiation factor 2B (eIF2B) (Frame et al., 2001), while the inactivation of eIF2B inhibits protein synthesis. Additionally, Akt phosphorylates TSC2 which removes the inhibition of TSC1/2 complex on the Ras homologue enriched in brain (Rheb) activity on mTORC1 (Zoncu et al., 2011).

While the exact mechanisms of how amino acids stimulate protein synthesis are unknown, recent work has shown that localization of mTORC1 to the lysosome has emerged as a critical regulatory point in the activation of mTORC1 by amino acids and exercise (Abou Sawan et al., 2018; Bar-Peled \& Sabatini, 2014; Hodson et al., 2017). Additionally, mTORC1 is found throughout the cytoplasm when amino acids are low, when stimulated the regulator-rag complex targets $\mathrm{mTORC} 1$ to the lysosomal surface to interact with GTPase Rheb (Abraham, 2010; Sancak et al., 2010). Furthermore, recent work has highlighted the implications of GATOR 1 and 2 on the recruitment of mTORC1 to the lysosome via recruitment by RAG A/B and C/D (Graber et al., 2019). Gap Activity towards RAG 1 (GATOR 1) is comprised of three subunits; Nitrogen permease regulatorlike 2 (NPRL2), Nitrogen permease regulator-like 3 (NPRL 3), and DEP domain containing 5 (DEPDC5) while GATOR 2 is a made of 5 subunits. When amino acids are high GATOR 1 and 2 form a super complex which allows for the RAG A/B and C/D 
activation therefore allowing full mTORC1 activation (Ho et al., 2016; Moro et al., 2016). If the GATOR 1 and 2 are not forming a complex, then GATOR can inhibit RAG activity thus preventing mTORC1 to reach the lysosome. Lastly, the most examined output of mTORC1 is the phosphorylation of the eukaryotic initiation factor 4E (4E-BP1) and the p70 ribosomal S6 kinase (S6K1) to promote protein synthesis. The hyperphosphorylation of 4E-BP1 prevents binding to eukaryotic initiation factor 4E (eIF4E) and the formation of 4E-BP1-eIF4E complex, resulting in the assembly of the eIF4F complex and translation initiation (Kimball \& Jefferson, 2010). Lastly, S6K1 activation by $\mathrm{mTORC} 1$ has been implicated in cap-dependent translation, translation elongation, and ribosomal biogenesis (Bentzinger et al., 2008; Kimball et al., 1999).

While it is well established that muscle protein breakdown is activated during wasting, whether suppressed protein synthesis regulation contributes to muscle mass loss during cachexia is less known. To date, only a few studies have examined muscle protein synthesis and breakdown rates in cancer patients (Dworzak et al., 1998; Emery et al., 1984; van Dijk et al., 2015; van Dijk et al., 2019). While there are only a few human studies, it has been concluded that changes in both basal and postprandial muscle protein synthesis and breakdown rates contribute to the muscle wasting observed in cachectic cancer patients, suggesting that the regulation of both are different (Brook et al., 2017; Horstman et al., 2016).

While a majority of our understanding of protein synthesis during cachexia is examined in murine models, protein synthesis rates are reduced in cachectic patients (Dworzak et al., 1998). We have routinely shown suppressed protein synthesis during the progression of cachexia in the $\mathrm{Apc}^{\mathrm{Min} /+}$ mouse, and this suppression corresponded to a reduction in muscle protein mTORC1 and IGF1 but were independent of Akt S473 (White, Baynes, et al., 2011). This early suppression of protein synthesis during cachexia did not correspond to an initial change in AMPK, since AMPK activity does not increase till late-stage cachexia (White, Baynes, et al., 2011). Interestingly, IL-6 receptor antibody was sufficient to attenuate muscle mass loss and AMPK signaling, however IL-6 was not sufficient to restore protein synthesis or mTORC1 (White, Baynes, et al., 2011). However, pyrrolidine dithiocarbonate (PDTC; STAT3 and P65 inhibitor) was sufficient in activating protein synthesis and reducing protein breakdown (Narsale et al., 2016).

Additionally, we have examined a role for systemic IL-6 in suppressing protein synthesis during cachexia (White et al., 2013). Furthermore, cytokine dosing is commonly used in cell culture to provide a high inflammatory environment. We have previously shown that IL-6 exposure has the capacity to decrease C2C12 myotube diameter and suppress mTORC1 signaling, and when AMPK is blocked the IL-6 suppression of protein synthesis is relieved (White et al., 2013). In the LLC tumor model, downstream targets of mTORC1; 4EBP-1 and rpS6, are suppressed during cachexia (Puppa, Gao, et al., 2014). Interestingly, gp130 loss did not improve the suppressed mTORC1 signaling, but did prevent muscle wasting (Puppa, Gao, et al., 2014). Furthermore, LLC tumor derived media on C2C12 myotubes suppressed protein synthesis which coincided with STAT3 and AMPK phosphorylation (Gao \& Carson, 2016). AMPK, but not gp130 inhibition rescued protein synthesis suppression. 
Collectively, these studies highlight that suppressed muscle protein synthesis and mTORC1 signaling is linked to IL-6/STAT3, but alone is not sufficient to restore protein synthesis during cachexia progression.

\section{Protein breakdown}

Muscle atrophy involves the upregulation of proteolytic systems to remove contractile proteins and organelles (Bonaldo \& Sandri, 2013). There are two main proteolytic systems involved in muscle atrophy: ubiquitin-proteasome and autophagylysosome. Additionally, calpain proteolysis has also been implicated in muscle atrophy (Huang \& Zhu, 2016). Calpains are Ca2+-dependent cysteine proteases which can cleave cytoskeletal protein for further breakdown at the proteasome. Lastly caspases are a family of proteolytic enzymes that are most known for their role in initiating apoptosis (Bell et al., 2016).

Ubiquitin-proteasome system (UPS) is the main mechanism for protein degradation (Lecker et al., 1999). The UPS is comprised of 3 enzymes that conjugate ubiquitin which are recognized for degradation. The first enzyme component is E1 (Ubactivating enzyme) which activates proteins after ATP cleavage. The ubiquitin is moved from E1 to E2 (Ub-carrier or conjugating proteins). E1 and E2 are enzymatic components that link the ubiquitin chain. The third enzyme E3 (Ub-protein ligase) is highly important as it recognizes a specific protein substrate and catalyzes the transfer of activated ubiquitin to a substrate chain. The degradation of proteins through the ubiquitin proteasome is a highly coordinated process and in skeletal muscle utilizes the musclespecific E3 ligases Atrogin-1/MAFbx and MuRF-1 which are controlled upstream by FOXO3a and were discovered initially through the studies examining starvation and fasting conditions. The binding of E3 to E2 elicits the transfer of ubiquitin from E2 to the substrate. These E3 ligases allow for targeted proteins to be recognized by the $26 \mathrm{~S}$ proteasome and ensure specificity of the UPS and additional adaptor proteins (Murton et al., 2008). In addition to inducing protein synthesis, Akt has the capacity to inhibit catabolic processes through the phosphorylation and inhibition of the FoxO. Akt can phosphorylate FoxO at Thr24, Ser 256, and Ser319, which prevents nuclear entry and activation of gene expression (Tzivion et al., 2011).

Cancer cachexia is commonly characterized by activated UPS in murine and human studies (Bossola et al., 2003; Lorite et al., 1998; Tisdale, 2009; Williams et al., 1999). Atrogin-1 and MuRF-1 are E3 ligases commonly investigated in cachectic skeletal muscle. During the progression of cancer cachexia, ATP-dependent protein degradation activity (Lorite et al., 1998), muscle E3 ligase expression, and ubiquitin conjugates are increased with the initiation of cachexia ( $<5 \%$ body weight loss) and are further increased with cachexia severity (White, Baynes, et al., 2011). Furthermore, we have shown that systemic IL-6 is associated to muscle Atrogin-1 mRNA during cachexia suggesting a link between inflammation and protein breakdown during cachexia (Baltgalvis et al., 2009). Forkhead transcription factors (FoxO) and the inflammatory transcription factor, NFKB, act on the promoters for Atrogin-1 and MuRF-1 respectively to stimulate their 
expression. FoxO and NFkB can accelerate muscle wasting by E3 ligase conjugation with atrogenes (Cai et al., 2004; Sandri et al., 2004). Interestingly, it has been hypothesized that E3 mRNA's might be a biomarker for excessive muscle proteolysis, however it is also important to understand that the induction of UPS is occurring under basal conditions and therefore is interesting to hypothesize how the system will respond to a stress.

Autophagy is a non-specific dynamic process used to remove damaged organelles, abnormal protein aggregates, or bulk contents (Ravanan et al., 2017). Autophagy can be initiated selectively and non-selectively by sequestering cytosolic substrates within a phagophore which is then conjugated with ubiquitination-linked proteins and degraded by a lysosome (Sanchez et al., 2012; Vainshtein \& Hood, 2015). Under basal conditions, autophagy is relatively low, however nutrient deprivation, cell stress, and chemical reagents are known to induce autophagy (Ravanan et al., 2017). While autophagy is constantly in flux; prolonged and excessive autophagy can lead to cell death. The process of autophagy (self-eating) is comprised of 3 types; macroautophagy, microautophagy, and chaperone-mediated autophagy (Klionsky, 2005). Macroautophagy consist of an autophagosome (double membrane vesicle) formed by portions of the cytoplasm. The autophagosome fuses with the lysosome to release the inner single membrane into the lumen. Microautophagy consist of the direct engulfment of cytoplasm at the lysosome. Lastly, the chaperone-mediate autophagy translocate unfolded proteins directly across the lysosome. Despite all of this, most autophagy conducted is considered macroautophagy. Autophagy is induced through the AMPK activation of ULK-1 and has been extensively investigated with numerous catabolic conditions including fasting (Sandri, 2010; Yan et al., 2012). AMPK has long been established as a critical regulator of both protein synthesis and protein degradation and induces both the ATP-independent autophagy lysosomal proteasome and the ATPdependent ubiquitin proteasome, respectively. Within the cell, AMPK phosphorylates ULKS555 to form a complex with ATGs and Beclin. The formation of this complex functions to cleave the C-terminus of ATG4 to produce LC3-1, which can then form LC3-II thus allowing for the formation of the autophagosome membrane. Furthermore, when activated, AMPK will phosphorylate tuberous sclerosis protein 2 (TSC2) and Raptor, which inhibits mTORC1 activity (Gwinn et al., 2008). AMPK activation is also a regulator of skeletal muscle degradation through the induction of proteasomal and autophagic-lysosomal proteolysis by inhibiting mTORC1 (Bolster et al., 2002), upregulation of E3 ligase expression via FOXO3a (Greer et al., 2007) and activation of ULK-1 (Lira et al., 2013). Activation of mTORC1 inhibits autophagy through the phosphorylation of multiple autophagy-related proteins that promote autophagy initiation and autophagosome nucleation. Additionally, ULK1 phosphorylation S757 suppresses the phosphorylation of ULK1 S555 thereby inhibiting AMPK. Lastly, transcription factor EB (TFEB) is a member of the leucine-zipper family of transcript factors that control lysosomal biogenesis (Martina et al., 2012). Recent evidence has shown TFEB overexpression induces autophagy, which has known effects to regulate mTORC1which will sequester in the cytoplasm and inhibit transcriptional activity (Bajaj et al., 2019). 
The role of autophagy in muscle wasting, not only in cancer cachexia, but in any disease has progression has gained great interest (Levine \& Kroemer, 2008). Recent work has shown increased autophagy in cachectic skeletal muscle from murine and humans' models (Pigna et al., 2016; Tardif et al., 2013). Furthermore, autophagy associated signaling was only induced in cachectic cancer patients, compared to cancer and noncancer patients, highlighting the potential specificity of autophagy occurring later in cachexia progression (Aversa et al., 2016). To further support this rational, during severe cancer cachexia, skeletal muscle AMPK is dysregulated and chronically activated which could have dire consequences regarding muscle proteostasis. We have known for years that during cancer cachexia progression autophagy associated proteins are increased which coincided with increased AMPK signaling (White, Baynes, et al., 2011). Furthermore, increasing autophagy activity exacerbates muscle wasting (Penna, Ballaro, Martinez-Cristobal, et al., 2019). Taken together, these studies highlight autophagy's role in muscle wasting with cancer cachexia.

\section{Skeletal muscle phenotype}

Skeletal muscle is comprised of contractile and metabolic properties that elicit responses to numerous physiological conditions (Schiaffino \& Reggiani, 2011). Skeletal muscle is comprised of heterogenous populations of muscle fibers that are characterized by type: type I (slow-twitch) and type IIA/B (fast-twitch) (Szent-Gyorgyi, 2004). Slow oxidative fibers; type I, are muscle fibers that have a high mitochondrial density, small myonuclear domain, fatigue resistant, weak force production, and red in appearance (Egan \& Zierath, 2013). Fast oxidative-glycolytic fibers; type IIa, are fatigue resistance, moderate mitochondrial density, moderate myonuclear domain, fatigue resistant, moderate force production, and red in appearance. Fast glycolytic; type IIb, have low mitochondrial density, large myonuclear domain, reaches fatigue prior to oxidative muscle, strong force production, and white in appearance. There are slight differences between the characterization of mice and human myosin isoforms, briefly mice have 1 slow myosin isoform (MHC I) and three fast myosin isoforms (MHC IIa, IIb, and IId) (Schiaffino \& Reggiani, 1994; Talbot \& Maves, 2016). In addition, muscle fiber typing is commonly used to understand the treatment's response on skeletal muscle phenotype. At baseline though, muscle types in mice are characterized as the following: soleus fibers are mostly type I and IIa, extensor digitorum longus (EDL) and tibialis anterior (TA) muscle are comprised mostly of IIb, and the gastrocnemius is comprised of mostly IIb, but more I than EDL and TA. Furthermore, the gastrocnemius has a red and white portion further allowing for the comparison of muscle phenotype within the same muscle.

Skeletal muscle phenotype aids in the response to nutrients, chronic disease, and activity (Egan \& Zierath, 2013). Unfortunately, most studies that compare the effect of muscle phenotype compare the treatment or study manipulation response in different muscle's without comparing the basal response between muscle (Deval et al., 2020; Sebastian et al., 2016). To account for the differential regulation, several studies compare a similar response in different muscle phenotypes. While this was not the purpose of these studies, it does leave open to interpretation if the response or lack of response can 
be attributed to the basal phenotype. When comparing oxidative vs glycolytic muscles, oxidative muscles exhibit elevated autophagy-lysosome proteins, without changes in the E3 ligase mRNA (de Theije et al., 2015). Additionally, red gastrocnemius muscle has increased nicotinamide phosphoribosyl transferase which is the rate-limiting enzyme in the NAD salvage pathway compared to white gastrocnemius, supporting the higher metabolism in oxidative compared to glycolytic (Brandauer et al., 2013). Increased oxidative capacity in red muscle compared to white muscle is supported by increased pgclalpha and Sirt mRNA expression (White, Baltgalvis, et al., 2011). Interestingly, mitochondrial fusion proteins were induced in glycolytic muscle, but mitochondrial fission was similar between groups further elucidating to the basal differences between muscle can differentially regulate their response to a stimulus.

Chronic disease, disuse, and inflammation are known to disrupt skeletal muscle fiber types size and composition (Ciciliot et al., 2013; Dumitru et al., 2018; Gosker et al., 2002; Hua et al., 2017). A myofiber's response to catabolic stimuli has been shown to be regulated by phenotype; slow-oxidative fibers are more susceptible to disuse atrophy, whereas fast-glycolytic muscles are more sensitive to cachectic stimuli. In cachectic cancer patients, MHC I and II are decreased, only in late-stage cancer (Johns et al., 2014). In the C26 model of cachexia, fiber types shifts to an increase in smaller fibers (Bonetto, Rupert, et al., 2016), and exhibits a reduction in the total number of all fiber types (Murphy et al., 2019; Roberts, Ahn, et al., 2013). Furthermore, in a genetic model of cancer, fiber type IIa/b were decreased (Hardee et al., 2016), and highlights that the changes in type II fibers occurs in the later stages of cachexia (Baltgalvis et al., 2010). Interestingly, in a pancreatic model of cancer cachexia, only type IIx/IIb were decreased (Talbert et al., 2019). Taken together, these studies highlight that cachexia progression disrupts skeletal muscle fiber types, with a decrease in all fibers at end stage cachexia. We have only just started to scratch the surface in investigating cachexia's effect on altering homeostasis in oxidative and glycolytic muscles. To date, we know that COXIV protein expression was decreased in severe white gastrocnemius compared to weight stable and red gastrocnemius (White, Baynes, et al., 2011). Reduced mitochondrial content has been shown in both oxidative and glycolytic hindlimb muscles (White, Baltgalvis, et al., 2011; White et al., 2012). Furthermore, mitochondrial fission mRNA was induced with cachexia and fusion mRNA was lowered, without differences between fiber type. Additionally, we have shown that BAX; a nuclear-encoded protein aids in piercing the mitochondrial membrane to elicit apoptosis, is elevated in cachectic soleus muscle without changes in red and white gastrocnemius muscle in tumor-bearing mice (Baltgalvis et al., 2010). Taken together, there is sufficient data supporting that cachexia differentially regulates oxidative and glycolytic fibers, however this has yet to be fully understood.

\section{Inflammation}

Chronic inflammation is an adverse sequalae of many chronic diseases. While the acute induction of inflammation in response to injury is necessary for recovery, long term exposure can result in metabolic dysfunction and disrupted protein turnover. Pro- 
inflammatory cytokines; interleukin-1 (IL-1), interleukin-6 (IL-6), tumor necrosis factor alpha (TNF- $\alpha$ ), and leukemia inhibitory factor (LIF) that are chronically elevated during cachexia are one of the initial triggers in inducing hypothalamus/pituitary and mitochondrial dysfunction (Cole et al., 2018). Furthermore, inflammation has been implicated as a key mediator of cancer-induced cachexia leading to the chronic elevation of skeletal muscle STAT3 and MAPK activity (Deans \& Wigmore, 2005), thereby disrupting mitochondria. The next few sections will discuss the importance of inflammatory signaling during cachexia progression and how gp130 is a regulatory point of interaction between the systemic environment and muscle wasting.

Glycoprotein-130 (gp130) is transmembrane protein ubiquitously expressed in most tissues. There are several circulating cytokines that react with gp130; IL-6, LIF, oncostatin M (OSM), ciliary neutrophilic factor (CNTF), and cardiotrophin-1 (CT-1), to elicit effects on most tissues (Ma et al., 2019). Gp130 resides in the membrane, therefore interactions must occur through ligand-receptor responses. Signaling activity occurs commonly by 1 of 3 mechanisms. Most commonly, the $\alpha$-subunit binding by either IL- 6 , IL-11, or CNTF triggers oligomerization with type 1 cytokine receptor $\beta$-subunit of gp130 (Ernst \& Jenkins, 2004). This reaction causes gp130-gp130 homodimerization to elicit intrinsic signaling. Another mechanism of action is the heterodimerization of gp130 to a $\beta$-subunit; for example, LIFR $\beta$ and OsMR $\beta$. Lastly, facilitation of the $\beta$-subunit can be dimerized upon ligand blocking by a soluble component. This process is called receptor conversion or trans-signaling meaning that a soluble receptor will bind to gp 130 . Our understanding of these biological responses is that they are often overlapping, however recent work has tried to highlight specificity of gp130 binding.

Our initial understanding of the effects of gp 130 were examined to determine the interplay between immune and hematopoietic cells (Kishimoto et al., 1994). Interestingly, the majority of our understanding of the role of gp130 is by examining the loss of associated cytokines (IL-6, LIF, IL-11), with the majority of gp130 understanding derived from blocking/activating IL-6 signaling, thus limiting our understanding of the implications of gp130 signaling (Hirota et al., 1999). Over 20 years ago, the loss of gp130 highlighted the necessary function of gp130 on embryonic development since gp130 loss is embryonic lethal (Yoshida et al., 1996). Even the post-natal loss of gp130 using cre-recombinase resulted in neurological, cardiac, hematopoietic, and immunological defects (Betz et al., 1998; Ernst \& Jenkins, 2004). However, in a physiological state that induces gp130 associated signaling, the loss of gp130 in the liver inhibited the progression of liver cancer (Hatting et al., 2015), and has been implicated as a way to combat chronic disease (Drogemuller et al., 2008; Molyneaux et al., 2003; Streetz et al., 2003). Alternatively, constitutive expression of gp130 induced cardiac hypertrophy (Hirota et al., 1999). Taken together, these studies highlight that the regulation of gp130 is a dynamic process and the activation or suppression of gp130 associated signaling is tissue and condition specific.

Gp130 is a regulatory point of interaction between systemic inflammation to JAK/STAT and MAPK signaling in skeletal muscle (White \& Stephens, 2011). The phosphorylation of associated Janus kinase (JAK) is sufficient to recruit domain 
containing cytoplasmic protein tyrosine phosphatase (SHP-2) thereby initiating the phosphorylation cascade of Ras-Raf-MEK-ERK. Additionally, the phosphorylation of JAK can result in the phosphorylation of STAT1/3. STAT-dependent transcriptional regulation of suppressor of cytokine signaling (SOCS) is a negative regulator of STAT signaling by binding to JAK thus inhibiting the phosphorylation of STAT. In skeletal muscle, we have previously induced gp130 loss by generating gp130-floxed on the myosin light chain cre-promoter (Fix et al., 2018). Gp130 loss decreased STAT3 protein expression by $75 \%$. Gp130 loss did not affect muscle fatigue, run to fatigue time, and COX activity in the gastrocnemius muscle (Fix et al., 2018). Interestingly, gp130 knockout mice had greater lean mass and body mass (Puppa, Gao, et al., 2014). Furthermore, gp130 loss induced mitochondrial fission 1 protein (FIS-1) and decreased mitofusion-1 (MFN-1) in crude gastrocnemius muscle, which was also reported in isolated mitochondria highlighting changes to mitochondrial dynamics by gp130. This was followed up by an increase in Beclin-1 and P62, without changes in LC3II/I ratio, identifying dysfunctional clearance of the autophagosome (Fix et al., 2018). We have shown in cell culture that the mitochondrial quality control dynamic changes are not directly attributed to decreased STAT3 highlighting the multifactorial nature of gp130. Lastly, Akt phosphorylation T308 was significantly induced in gp130 knockout mice without effecting downstream mTORC1 signaling (Puppa, Gao, et al., 2014). It can be hypothesized that a further induction of $\mathrm{mTORC} 1$ signaling in the basal condition is unlikely to be affected by gp130 loss alone, especially since AMPK signaling was not altered. Taken together, these data highlight that gp130 loss has regulatory function on mitochondrial dynamics with minimal alterations to Akt/mTORC1/AMPK signaling following a brief fast.

In chronic disease induced cachexia, gp130 protein expression was elevated in Kaposi's sarcoma; a common cancer in AIDs patients (Morris et al., 2008). Furthermore, in several models of cancer induced cachexia, gp130 downstream signaling was also upregulated (Bonetto et al., 2012; Puppa, Gao, et al., 2014; White, Baynes, et al., 2011). Interestingly, gp130 loss in neurons reduced LLC induced pain (Andratsch et al., 2009). Several studies have implicated the role of gp130 signaling on preventing and/or treating cancer induced cachexia. First, skeletal muscle-specific STAT loss prevented LLC induced muscle wasting and grip strength loss, without altering tumor mass (Silva et al., 2015). Interestingly, in a genetic model of cancer induced cachexia ( $A p c^{\text {Min/+ }}$ mice), IL-6 receptor antibody preserved some body weight and muscle mass loss (White, Baynes, et al., 2011). Furthermore, IL-6 receptor antibody was sufficient in reducing STAT3 and AMPK signaling without rescuing the suppression of mTORC1. We have previously administered PDTC (anti-inflammatory) to mice injected with LLC cells, while muscle STAT3 was reduced muscle mass was not preserved (Puppa, Gao, et al., 2014). Interestingly, we have previously reported muscle gp130 loss prevented LLC induced muscle wasting and restored STAT3 and MAPK signaling, however the mTORC1 downstream signaling was not rescued (Puppa, Gao, et al., 2014). Lastly, IL-6 induced mitochondrial dysfunction and fatigue requires skeletal muscle gp130 (VanderVeen et al., 2019). Interestingly, cachexia has known disruptions in mitochondrial function prior to muscle wasting (Brown et al., 2017) and theses disruptions to the muscle mitochondria are known to induce AMPK, thereby accelerating muscle wasting (VanderVeen et al., 
2017). Given that muscle gp130 can regulate mitochondrial quality control (Hardee, Counts, et al., 2018), whether these are linked to cachexia disruption of protein turnover by feeding are warranted.

Cancer cachexia is a multifactorial wasting condition and can lead to systemic and tissue specific metabolic dysfunction (Fonseca et al., 2020; Kunzke et al., 2020). Indirect calorimetry and energy expenditure have long been used to anticipate and/or assess metabolic dysfunction (Lam \& Ravussin, 2016). Indirect calorimetry provides identification of fuel source through exchange of gases, allowing for a noninvasive method to indirectly identify oxygen consumption, carbon dioxide production, predominate fuel source, and energy expenditure. We have known for quite some time that lung cancer patients exhibit increased energy expenditure and the association to body weight loss (Eden et al., 1984). It is well known that the tumor's metabolic instability can result in tumor cells reprogramming or inability to respond to treatment (Vanhove et al., 2019), likely resulting in discrepancies between decreased survival in whole body hypometabolic (Jatoi et al., 1999; Jebb et al., 1994) or hypermetabolic patients (Vazeille et al., 2017). These discrepancies have led to the inability to provide therapeutic options to mitigate metabolic disruptions that are greatly influenced by tumor stage and therapeutic treatments. Thus, highlighting the need to further understand the metabolic dysfunctions attributed to lung cancer. The following sections will highlight the disruptions that occur during cancer cachexia with the mitochondria and the important regulation of AMPK in these processes.

\section{Mitochondria}

Mitochondria are highly plastic, dynamic, adaptable, and necessary for proper skeletal muscle function. Mitochondria are often termed the "powerhouse" of the cell, while not their only function, they predominantly manage ATP production. In addition, mitochondria regulate signaling for protein turnover, autophagy, and apoptosis (Romanello \& Sandri, 2010). The mitochondria are a complex network, having two distinct subfractions; subsarcolemmal (SS) and intermyofibrillar (IMF) fractions (Attardi $\&$ Schatz, 1988). The SS accounts for $20 \%$ of the muscle's mitochondria and is located

directly under the plasma membrane to provide the energy to transport substrates and initiate signaling. Next, the IMF is located between myofibrils to provide ATP for muscle contraction. Both subfractions are necessary for proper mitochondria function. To meet energy demands, mitochondria are upregulated to compensate. The increase in mitochondrial size or number; mitochondrial biogenesis, occurs in response to stress (Jornayvaz \& Shulman, 2010). The stress imposed on the muscle can active the energy sensor AMPK to provide energy for mitochondrial biogenesis by suppressing protein synthesis. Furthermore, mitochondria regulate protein degradation through mitochondrial fission to activate AMPK thereby regulating FOXO3 (Romanello et al., 2010). The increase in AMPK induces peroxisome-proliferator gamma-activated receptor coactivator (PGC-1 $\alpha$ ) (Jager et al., 2007). The increase in PGC-1 $\alpha$ regulates nuclear encoding mitochondrial proteins (NUGEMPs) (Wu et al., 1999), but also regulates itself by mTORC1 activity (Cunningham et al., 2007). The importance of mTORC1 in 
mitochondrial homeostasis is further supported by the inhibition of mTORC1 that reduced muscle oxidative capacity and function (Morita et al., 2017; Schieke et al., 2006).

Mitochondrial dynamics is regulated through mitochondrial fission and fusion (Yaffe, 1999). First, mitochondrial fission occurs through the separation of mitochondria into two. Mitochondrial fusion occurs by having two mitochondria coming together to form one. Mitochondrial fission is regulated by dynamin related protein-1 (DRP1) and fission 1 protein (Fis1). DRP1 locates on the outer mitochondrial membrane to interact with Fis 1 an adaptor protein and mitochondrial fission factor to elicit fission (Benard \& Karbowski, 2009). DRP1forms a ring at fission sites and constricts by GTP hydrolysis. A recent study has shown that DRP1 loss in skeletal muscle induces abnormal calcium handling, increased mitochondrial calcium uniporter (MCU), and resulted in muscle weakness and atrophy (Favaro et al., 2019), highlighting the necessary function of DRP1. While the mechanism is not completely known, Fis 1 inhibition has been shown to reduce autophagy (Romanello et al., 2010) and has been implicated in the maintenance of health by targeting dysfunctional mitochondria (Iglewski et al., 2010). Mitochondrial fusion is regulated by outer-membrane GTPases mitofusin 1 and 2 (Mfn1/Mfn2), and innermembrane GTPase optic atrophy protein 1 (OPA1). Mfn1/2 work by tethering mitochondria through and OPA1 acts as the anchor during fusion (Zorzano \& Claret, 2015). Mfn1 and 2 have suggested to have overlapping function and their activity suggest to being tissue specific. For example, both Mfn 1 and 2 are found in skeletal muscle, but Mfn2 is more active (Santel et al., 2003). Inhibition of mitochondrial fusion in mice leads to decreases in mitochondrial DNA and ultimately muscle atrophy (Chen \& Chan, 2010). Furthermore, Mfn2 has been shown to be regulated by PGC-1 $\alpha$ (Soriano et al., 2006). Taken together mitochondrial dynamics is a very plastic process and has been implicated as regulatory point in chronic disease (Chan, 2020). Mitochondrial loss and dysfunction are prominent features of muscle wasting (Argiles et al., 2015; Gamboa et al., 2016; Picca et al., 2017; VanderVeen et al., 2017). In addition, cachectic muscle exhibit decreased mitochondrial control (Marzetti et al., 2017) and has been shown in both oxidative and glycolytic hindlimb muscles (White, Baltgalvis, et al., 2011; White et al., 2012). Interestingly, we have shown insulin resistance in cachectic mice (Lombardi et al., 2012), suggesting a link between the inability to clear glucose and dysfunctional mitochondria.

Recent work has identified that LLC mice exhibit disrupted whole body insulin and glucose signaling prior to wasting (Han et al., 2020). In addition, skeletal muscle mitochondrial dysfunction onset occurs prior to muscle wasting (Brown et al., 2017). Taken together, these studies identify that muscle and whole-body metabolism associated disruptions are likely contributing to the wasting progression. Furthermore, we have consistently shown cachexia decreases mitochondrial fusion with a corresponding increase in fission (White, Baltgalvis, et al., 2011), suggesting a pivotal role in the regulation of skeletal muscle mass. Others have shown cachexia decreases muscle oxidative capacity, but was not associated to altered ATP production (Julienne et al., 2012). Furthermore, ATP production is decreased in tumor-bearing mice (Tzika et al., 2013), likely contributing to increased fatigue during cachexia. However, PGC-1 $\alpha$ 
transgenic mice were not sufficient to prevent muscle wasting despite an increase in mitochondrial content (Wang et al., 2012). Interestingly, overexpression of PGC-1 $\alpha 4$ was able to prevent severe cachexia progression and improve glucose metabolism and force production (Ruas et al., 2012). Furthermore, overexpression of mitofusin partially attenuated muscle mass loss highlighting the therapeutic potential of restoring mitochondrial content and dynamics (Xi et al., 2016). Systemic inflammation has been implicated in the regulation of mitochondrial dysfunction. During cachexia progression, IL-6 receptor antibody given after the initiation of cachexia was sufficient to lower mitochondrial fission and increase fusion, while also increasing mitochondrial biogenesis (White et al., 2012). In addition, systemic inhibition of NFאB and MAPK was sufficient to prevent body wasting, muscle mass loss, tumor burden, improve strength, and improve mitochondrial complex activity (Fermoselle et al., 2013). The use of these systemic inhibitors was sufficient to decrease tumor mass or number thus making it difficult to understand the regulation of these systemic inhibitors on muscle mitochondria. Taken together, mitochondria's role in skeletal muscle mass during cancer cachexia has only minimally been explored and requires further understanding.

Metabolism is a very broad term and encompasses a range of chemical reactions in response to fuel availability to maintain cell homeostasis (DeBerardinis \& Thompson, 2012). 5' adenosine monophosphate-activated protein kinase (AMPK) plays a crucial role in energy metabolism and cellular homeostasis (Romanello et al., 2010; Romanello \& Sandri, 2015). AMPK is a highly functional protein complex activated by nutrient deprivation, hypoxia, and toxins that disrupt mitochondrial transport chain (Viollet et al., 2010). Additionally, the duration of AMPK activity is critical in understanding the mechanism of action and potential implication. Chronic elevated AMPK signaling; specifically in skeletal muscle during aging (Mulligan et al., 2005) cancer cachexia (White, Baynes, et al., 2011) and COPD (Balnis et al., 2020) has been implicated as a negative consequence of the disease and therapeutic strategies to circumvent this chronic activation has been sought after for decades. Alternatively, exercise is a known acute inducer of AMPK, however the induction of AMPK is dependent on the exercise intensity and duration (Kjobsted et al., 2018). It is important to distinguish between the adverse chronic consequences of elevated AMPK compared to the necessary AMPK induction for exercise adaptations.

AMPK is a heterotrimeric enzyme comprised of two alpha $(\alpha)$ subunits ( 1 and 2$)$, two scaffolding beta $(\beta)$ subunits (1 and 2$)$, and three regulatory gamma $(\gamma)$ subunits $(1,2$ and 3) (Fuchsberger et al., 2016). The phosphorylation of AMPK T172 involves physiological activity by the AMPK alpha catalytic subunit and is a major site for AMPK's activity. To date, only 3 subunit combinations exist in humans ( $\alpha 1 \beta 2 \gamma 1$, $\alpha 2 \beta 2 \gamma 1$, and $\alpha 2 \beta 2 \gamma 3$ ) (Birk \& Wojtaszewski, 2006) and 5 have been reported in mice $(\alpha 2 \beta 2 \gamma 1, \alpha 2 \beta 2 \gamma 3, \alpha 1 \beta 2 \gamma 1, \alpha 1 \beta 1 \gamma 1, \alpha 1 \beta 1 \gamma 1)$ (Kjobsted et al., 2018). Predominate expression combination for both human and mouse consist of $\alpha 2 \beta 2 \gamma 1$. The $\alpha$ subunit of AMPK can be activated by liver kinase B1 (LKB1), calcium/calmodulin-dependent kinase 2 (CaMKK2), TGF $\beta$-activated kinase 1 (TAK1), protein phosphatase $2 \mathrm{~A}$ (PP2A), protein phosphatase $2 \mathrm{C}(\mathrm{PP} 2 \mathrm{C})$, and magnesium dependent protein phosphatase 1E (PPM1E) (Jeon, 2016). Increased energy induces low AMP/ATP and ADP/ATP ratios, 
thereby allowing phosphatases to access T172 keeping it unphosphorylated, however when levels of AMP and ADP increase and bind to cystathionine $\beta$-synthase (CBS3) at the gamma subunit, this prevents the phosphatases from binding to T172. AMP and ADP binding to CBS3 stimulate LKB-1 to phosphorylate AMPK T172 on the $\beta$ subunit (Scott et al., 2004). Furthermore, calcium activates AMPK through CaMKK2 activity. Insulin inhibits AMPK by phosphorylating AKT which phosphorylates one of two AMPK $\alpha$ subunit sites therefore blocking upstream kinases from phosphorylating T172. Additionally, the hormone leptin acts similar as AKT but through p70S6K. Lastly, protein kinase A (PKA); activated by the binding of cyclic AMP to its R subunit, inhibits AMPK phosphorylation sites on the $\alpha$ subunit. Additionally, increases in reactive oxygen species (ROS) regulate AMPK, the mechanism is thought to occur through glucose depletion and decreased antioxidant capacity however the exact mechanisms still remains elusive (Jeon, 2016). AMPK can also be regulated by activating the peroxisome proliferator activated receptor gamma coactivator 1alpha (PGC1alpha)-hypoxia inducible factor 1alpha (HiF1). This activity activates the sestrin family of proteins to induce TSC1/2 signaling thereby inhibiting downstream target mTORC1. Altogether, there are several mechanisms of action to elicit AMPK and the process is very complex, thus understanding AMPK's specificity is crucial to understanding and interpretating a treatment or conditions response by altering AMPK.

Once activated, AMPK elicits several downstream cascades. First, AMPK has known regulation on lipid metabolism by inhibiting new fatty acid synthesis through phosphorylating acetyl-Cao carboxylase 1 (ACC1) to disrupt the fatty acid synthesis rate limiting step of malonyl-Cao. The second method of inhibiting fatty acid synthesis is the activity of sterol regulatory element-binding protein 1c (SREBP1c) to promote lipogenic enzymes. Additionally, triglyceride synthesis is catalyzed by glycerol-3phopshate acyltransferase which inhibits AMPK, while AMPK inhibits cholesterol synthesis through blocking phosphorylation of HMG-CoA reductase (Motoshima et al., 2006). AMPK's known activity to promote lipid catabolism is induced by carnitine palmityltransferase-1 (CPT-1) which increases from AMPK's phosphorylation of ACC2. ACC2 is localized to the outer mitochondrial membrane to inhibit production of malonylCoA that in turn will inhibit the allosteric inhibitor of CPT-1 therefore inducing fatty acid oxidation (Hardie \& Pan, 2002). Additionally, AMPK has regulation on glucose uptake through TCB1D1 phosphorylation. Tab-GTPase-activating protein (TCB1D1) is a RabGTPase activating protein that inhibits GLUT4 through rab protein inactivity (Miinea et al., 2005). Phosphorylation of TCB1D1 dissociates from GLUT4, thereby aiding in GLUT4's translocation. Taken together, AMPK is the control center for several metabolic associated perturbations to induce fatty acid lipolysis.

The role of AMPK has long been sought after given its multiple mechanisms of action and several inputs. Understanding the mechanisms of AMPK have been extensively reviewed regarding the importance and impact of AMPK (Fuchsberger et al., 2016; Jeon, 2016; Kjobsted et al., 2018; Steinberg \& Jorgensen, 2007). First, it is necessary to understand that whole body AMPK $\alpha 1 / \alpha 2$ loss is embryonic lethal and $\beta$ subunit 1 and 2 loss are embryonic lethal (Quinn et al., 2010) identifying the importance of $\alpha$ and $\beta$ for development. However, AMPK $\alpha 2$ whole body loss is not lethal, which is 
likely due to other subunits function given that AMPK $\alpha 1$ expression was present (Viollet, Andreelli, Jorgensen, Perrin, Geloen, et al., 2003). Additionally, while AMPK alpha 2 loss did impair fat oxidation during exercise recovery, it remains unclear if AMPK $\alpha 2$ is essential or if there is feasibility of other subunits to upregulate when one subunit is lost (Fritzen et al., 2015). Additionally, despite observing a 50\% decrease in AMPK T172 phosphorylation in skeletal muscle, whole body AMPK $\alpha 2$ loss exhibited increased muscle AMPK $\alpha 1$ activity suggesting a potential confounder when investigating the role of AMPK (Jorgensen et al., 2007). Furthermore, whole body $\beta 1 / 2$ loss mice exhibit decreased respiratory exchange ratio and suppressed glucose update (O'Neill et al., 2011), highlighting the specificity of the beta subunit function on whole body metabolism. It is important to mention, that the authors directed the disruption on the systemic adrenergic response and not specifically a role from skeletal muscle. Lastly, whole body loss of $\gamma 3$ subunit had capacity to decrease AMPK activity at rest, during exercise, and fasting without altering insulin sensitivity under these conditions, identifying the regulation of insulin on AMPK might be through another subunit (Barnes et al., 2004). Overall, AMPK loss of any subunit has the potential to disrupt normal metabolism, and it is necessary to consider the subunit manipulation and tissue of interest to fully determine the role of AMPK's function.

The role of AMPK in skeletal muscle has long been investigated in chronic disease and in response to exercise. A recent review has extensively examined the effects and implications of AMPK loss (KO) in skeletal muscle (Kjobsted et al., 2018; Viollet et al., 2009). Disrupted skeletal muscle AMPK $\alpha$ or $\beta$ subunits are commonly investigated. Whole body AMPK $\alpha 2 \mathrm{KO}$ was sufficient to reduce AMPK activity in skeletal muscle with only modest changes in AMPK $\alpha 1$ (O'Neill, 2013). Interestingly, whole body AMPK $\alpha 1 \mathrm{KO}$ mice did not alter muscle AMPK activity, identifying that $\alpha 2$ subunit is the predominant and subunit 2 is the predominant source of AMPK's activity (Viollet, Andreelli, Jorgensen, Perrin, Flamez, et al., 2003). It is necessary to mention that when a metabolic demand is placed on the mouse by high fat diet, insulin resistance is exacerbated by AMPK $\alpha 2$ KO (Fujii et al., 2008), however the loss of AMPK $\alpha 2$ in skeletal muscle was not sufficient to accelerate insulin resistance alone (Beck Jorgensen et al., 2009). Furthermore, whole body AMPK $\beta 2$ KO was sufficient to decrease basal muscle AMPK activity, however $\beta 1$ and $\alpha 1$ subunits were increased to compensate (Steinberg et al., 2010). It is important to mention that whole body $\beta 1 / 2$ loss was sufficient to make AMPK activity non-detectable at rest and during exercise, therefore identifying the importance of $\beta 1$ and $\beta 2$ subunits. Additionally, deletion of both $\beta$ subunits decreased $\alpha$ subunits resulting in disrupted muscle mitochondrial homeostasis and an impaired response to exercise, without altering myosin heavy chain content (O'Neill et al., 2011).

While several studies have investigated the role of whole-body AMPK loss effects in muscle, several of these studies have limited interpretation to do the systemic wide loss of AMPK, thus limiting our understanding of AMPK's functional disruptions. Despite this, several studies exhibit decreased functional outcomes either run to fatigue, daily wheel distance, or cage activity in AMPK $\alpha 2$ heart and skeletal muscle KO mice (Maarbjerg et al., 2009; Morissette et al., 2014; Zwetsloot et al., 2008). Furthermore, 
muscle-specific AMPK $\alpha 2$ loss was sufficient to decrease running capacity, without disrupting fatty acid oxidation during endurance exercise (Miura et al., 2009).

Interestingly, muscle AMPK $\alpha 2$ KO decreased VEGF expression suggesting AMPK's role in adrenergic pathways, however muscle AMPK $\alpha 2 \mathrm{KO}$ did not inhibit the exercise response thus suggesting that under basal conditions the blood flow to the muscle might be regulated through AMPK (Zwetsloot et al., 2008). Furthermore, muscle-specific AMPK $\beta 1 / 2 \mathrm{KO}$ decreased muscle fiber size, reduced capillary density, and lowered nitric oxide synthase (Thomas et al., 2014). Lastly, the $\gamma 3$ subunit of AMPK is predominately expressed in glycolytic fibers, suggesting AMPK's differential role in muscle types (Barnes et al., 2004). Studies utilizing $\gamma 3 \mathrm{KO}$ show decreased glycogen and PGC1 $\alpha$ mRNA expression during fasting and exercise, suggesting a regulatory role of AMPK gamma 3 in glycogen stores and utilization (Canto et al., 2010).

Cancer cachexia is a known chronic inducer of AMPK. Elevated skeletal muscle AMPK has been reported in cancer patients and in several murine models of cancer induced cachexia (Hall et al., 2018; Puppa, Gao, et al., 2014; Segatto et al., 2017; Talbert et al., 2019; White et al., 2013). We have previously shown in $A p c^{\mathrm{Min} /+}$ mice that AMPK activity and phosphorylation are induced with increasing cachexia severity (White, Baynes, et al., 2011). We have previously shown that IL-6 receptor antibody in the cachectic environment was sufficient to improve mitochondrial dynamics and biogenesis highlighting the impact of IL-6 on driving muscle wasting (White et al., 2012). Interestingly, IL-6 was sufficient to chronically induce AMPK in wildtype mice, highlighting a role for inflammation in the chronic activation of AMPK in skeletal muscle (White et al., 2013). Furthermore, in a C26 model of cancer cachexia, AMPK agonist AICAR was able to impair tumor growth and prevent skeletal muscle mass loss (Hall et al., 2018). While metformin, AMPK activator, did not alter tumor mass, it was sufficient to prevent the induction of AMPK in skeletal muscle (Oliveira \& GomesMarcondes, 2016). Recent work has highlighted SNARKs; an AMPK kinase, therapeutic role in regulating muscle wasting with cancer cachexia (Alves et al., 2019), however further work is needed to fully understand the implication of SNARK during cachexia progression. However, chronic metformin use has recently been shown to induce muscle mass loss (Kang et al., 2021), suggesting negative consequences on muscle mass with long-term AMPK activation. Taken together, the role of AMPK is not yet clear and recent articles highlight the importance of AMPK activation has preventative measure vs therapeutic treatment. Lastly, there has been sufficient evidence to identify that AMPK is elevated during cachexia and is associated to elevated inflammation, however there is little evidence in determining the mechanism of AMPK loss during cancer cachexia.

\section{Nutrition}

\section{Overall nutrition}

An oncology patient's quality of life is becoming an important topic to consider due to increased survival from early detection and successful therapeutic treatments. The 
nutritional status of the cancer patient is an important consideration and nutritional interventions should be initiated to prevent or offset malnutrition (van Bokhorst-de van der Schueren, 2005). Furthermore, nutritional status can greatly impact skeletal muscle homeostasis, especially since cancer patients (pre-cachexia) exhibit malnutrition. Therefore, there have been several studies to determine if nutritional supplemental is sufficient to prevent and treat cancer cachexia. Briefly, oral supplementation is often administered if the patient is unable to meet the necessary nutritional requirements from their normal food consumption (Ravasco et al., 2003) and has been suggested as an additional method to prevent further weight loss in cachectic cancer patients (Fearon et al., 2003). Eicosapentaenoic acid (EPA) supplementation has been implicated as a potential therapeutic due to its anti-inflammatory properties, immune enhancing effect, and attenuation of lipid-mobilizing factor (van Bokhorst-de van der Schueren, 2005), however future work is needed to determine if EPA supplementation is a viable therapeutic to offset wasting. Moreover, antioxidants have long been sought after to offset chemotherapy toxicity to prevent wasting. While cachexia is termed irreversible with nutritional support, we need to take a step back and have a better understanding if 1) the patient is malnourished 2) the composition of the patient's diet 3) the patient's dietary habits (are there periods of fasting) to fully understand the implications of nutritional status on cancer cachexia progression.

The first sign of disrupted protein synthesis in cachectic cancer patients was reported in 1984. Cachectic cancer patients showed reduced muscle protein synthesis even in the fed condition, without changes in whole body protein synthesis (Emery et al., 1984). While whole body protein turnover measurements in cachectic cancer patients have been shown to be elevated at baseline, the whole body and muscle anabolic response to feeding is suppressed in cachectic cancer patients (van Dijk et al., 2015). To circumvent the issues with performing tracer studies in cachectic cancer patients, most data related to muscle protein synthesis has been collected in rodent models of cancer cachexia. Taken together, patient nutritional status should be accounted for given the impact of nutrients on regulating skeletal muscle mass, and the next few sections will highlight the regulation of feeding and fasting, and how cachexia might alter these responses.

\section{Feeding}

Skeletal muscle's capacity for protein synthesis and degradation is highly sensitive to the local and systemic stimuli that are integrated intracellularly through mTORC1 and AMPK signaling (Frost \& Lang, 2007). Oscillations in protein synthesis and breakdown occur throughout the day due to feeding, fasting, and activity (Breen \& Phillips, 2011). These oscillations in feeding and fasting behaviors arise from the regulation by the central nervous system. Leptin and ghrelin are two opposing hormones regulating energy balance (Klok et al., 2007). Leptin is produced and released from fat cells in response to food intake, the increase in leptin elicits a reduction in appetite. Alternatively, ghrelin is produced and released mainly from gastrointestinal mucosa (Sakata \& Sakai, 2010), but has been located in the pancreas, ovary, adrenal cortex, and 
the brain. The release of ghrelin promotes food intake. Furthermore, feeding elicits an increase in circulating glucose to promote insulin release by the pancreas thereby translocating GLUT4 to the muscle's membrane to remove circulating glucose (Richter \& Hargreaves, 2013) and circulating insulin can also directly bind to IGF-1 resulting in a confirmational change to induce downstream phosphorylation of IRS/AKT/mTORC1 (Boucher et al., 2014). Feeding can directly target mTORC1 by increasing the amino acid pool to promote protein synthesis (Liu \& Sabatini, 2020). Additionally, a common suppressor of AMPK is nutrient stimulation through the consumption of glucose, a mixed meal (feeding), or amino acids (Koh et al., 2008; Long \& Zierath, 2006).

Feeding has been widely investigated as a regulator of mTORC1 and AMPK. The consumption of glucose or a mixed meal will induce a glucose and insulin response activating the IGF-1/AKT signaling cascade (Sengupta et al., 2010). AKT will then phosphorylate TSC2 releasing its inhibition of mTORC1(Sengupta et al., 2010). AMPK and mTORC1 negatively regulate one another, for example during energy demand AMPK is activated thereby suppressing energy consuming pathways (mTORC1) and stimulating energy generating pathways (ULK-1, FOXO3a) (Koh et al., 2008; Long \& Zierath, 2006; Sanchez et al., 2012). Muscle mTORC1 signaling is activated with both exercise and feeding and will phosphorylate ULK-1 at Ser 757 and inhibit the ULK-1 activation of downstream autophagy signaling (Laker et al., 2017; Sanchez et al., 2012). While the above-mentioned physiological changes to feeding and fasting occur throughout the day in healthy populations, significant barriers still exist in our understanding of how cachexia progression disrupts the feeding response.

While cancer patients demonstrate an increase in muscle protein synthesis after protein ingestion, there is sufficient evidence to support that the responsiveness to protein administration is strongly reduced during cancer cachexia (Deutz et al., 2011; Emery et al., 1984). Interestingly, following tumor resection, the post-prandial anabolic response was restored highlighting the impact of the tumor environment on the feeding response in muscle (Williams et al., 2012). Thus, this anabolic resistance to feeding attenuates the postprandial rise in muscle protein synthesis and most likely contributes to the loss of muscle mass observed in cancer cachexia. In pre-clinical models, our laboratory has demonstrated altered protein turnover occurs through suppressed anabolic signaling via mTORC1 and coinciding with this suppression is the chronic activation of AMPK (White, Baynes, et al., 2011). Recent work from our lab, has shown that mTORC1 signaling exhibits diurnal fluctuations in response to changes throughout the day in feeding and activity (Counts et al., 2020). Interestingly, the cachectic environment disrupts these diurnal fluctuations, and suggest a link between disruptions in feeding and activity behaviors to the progression of cachexia and altered mTORC1 signaling (Counts et al., 2020). Furthermore, a bolus of glucose was sufficient to improve muscle mTORC1 signaling and lower AMPK in cachectic skeletal muscle, albeit to a lower extent compared to wildtype controls (White et al., 2013), thus providing evidence that cachectic muscle has the capacity to respond to an anabolic stimulus. Following low dose leucine supplementation in LLC mice's diet, muscle mass loss was not preserved, but leucine was able to prevent disrupted mitochondrial biogenesis signaling (Lee et al., 2019). Interestingly, ghrelin administration in clinical trials has provided sparing of body 
weight loss (Ali et al., 2013), and might have therapeutic potential in patients experiencing anorexia (Guillory et al., 2013). In a transgenic animal model, ghrelin induction prevented fasting, and disuse induced atrophy (Porporato et al., 2013). Additionally, when administered, ghrelin prevented body weight and muscle mass loss during cancer cachexia progression in LLC mice, likely through increased food consumption that resulted in improved proteins synthesis and lowered protein degradation (Chen et al., 2015). Recent work has highlighted ghrelin's impact on preventing white adipose tissue inflammation, increased food intake, and preserving fat mass in cachectic mice (Liu et al., 2020). However, it is currently unknown if ghrelin can be used as a therapeutic to rescue or attenuate cancer induced cachexia but provides as a promising therapy for anorexia associated cachexia. Taken together, skeletal muscle regulation by cachexia in the presence of nutrients is complex and has yet to be fully elucidated.

\section{Fasting}

Fasting is known to induce AMPK thereby activating E3 ligases, while simultaneously suppressing IGF-1 signaling in vitro (Sandri et al., 2004). While several studies have examined the regulation of fasting on metabolism and associated muscle signaling, it is essential to understand that most pre-clinical studies using fasting use a long-term fast (24-48hrs) (de Theije et al., 2018), not to be confused with starvation. They are distinguishable because starvation studies typically encompass a $72 \mathrm{hr}$ fast or are related to animal hibernation. Nevertheless, fasting in pre-clinical models elicits an energy stress demand resulting in increased muscle AMPK signaling thereby inducing autophagy and E3 ligase activity which coincides with mTORC1 suppression (Bujak et al., 2015). Increased AMPK activity during fasting has the capacity to induce the ratio of ATP/ADP resulting in glucose metabolism, autophagy and lipid oxidation in order to replenish ATP stores (Herzig \& Shaw, 2018). Interestingly, fasting has been implicated in increasing AMPK mediated autophagy (Bagherniya et al., 2018; Dethlefsen et al., 2018). While the E3 ligase activation of AMPK has been considered a negative regulator of muscle mass, the autophagy response to fasting has been implicated as a method for cellular renovation and homeostasis (Bagherniya et al., 2018). In addition, IL-6 has also been implicated in response to fasting (Gudiksen et al., 2017). A brief fast was sufficient to increased muscle IL-6 mRNA without altering AMPK or P38 phosphorylation suggesting an early event in the fasting regulation of muscle protein turnover. Furthermore, fasting induced circulating IL-6 corresponded with increased plasma free fatty acids and reduced respiratory exchange ratio highlighting the fasting induction's role of lipolysis might be regulated by IL-6 (Wueest et al., 2014). Taken together, the effect of fasting on skeletal muscle mass regulation has been largely attributed to AMPK induced E3 ligase and autophagy signaling; however, less is understood about the role of cancer cachexia during fasting.

While repeated fasting; resulting in reduced caloric intake has been proposed to have beneficial effects in aging populations (Stekovic et al., 2019) and some cancers (Nencioni et al., 2018), less is understood about an acute fasting's role in the regulation 
of protein turnover in cachectic muscle. Our understanding of the skeletal muscle's signature in the cancer environment is examined following a short term fast. While this is beneficial in understanding the basal condition of muscle in the cachectic environment, some studies report a reduction of $20 \%$ in food intake at the end of life in cachectic animals therefore these studies are now investigating an anorexia model (Samuels et al., 2001) further confounding the data. Therefore, greater clarity within the literature is needed along with improved animal techniques. To date, our lab and others have demonstrated that the cachectic environment elevates skeletal muscle AMPK following a 5 hour fast, showing that a very short period of fasting is capable of inducing AMPK in the cancer environment and suggestive of metabolic dysregulation (Frost \& Lang, 2007). Concomitant with the induction of AMPK, our laboratory has demonstrated mTORC1 suppression during cancer cachexia, signifying a role for AMPK in the suppression of anabolic signaling (Hardee, Fix, et al., 2018; Hardee et al., 2016; White et al., 2013). However, significant barriers still exist in our understanding of cachectic skeletal muscle's protein turnover response by fasting.

\section{Skeletal Muscle Contraction}

One of skeletal muscle's main function is to elicit muscle contractions to generate force and movement for locomotion, breathing, and swallowing to survive. Skeletal muscle consists of multinucleated muscle fibers further dividing into myofibrils. Ttubules form deep invaginations within myofibrils that surround the muscles membrane. Myofibrils contain contractile proteins, thick and thin filaments (Squire, 2019). Thick filaments are large protein myosin and thin filaments are composed of actin, tropomyosin, and troponin (Ohtsuki \& Morimoto, 2008). To elicit a contraction, an action potential travels to the motoneuron thereby depolarizing and resulting in the voltagegated calcium channels to open. The influx of calcium releases acetylcholine at the neuromuscular junction where acetylcholine will bind to the nicotinic receptors thereby eliciting an action potential in the muscle fiber. This action potential reaches the muscle cell membrane where the action potential is spread down the t-tubule to reach the interior of the muscle fiber. The t-tubule receptors will undergo a confirmational change that interacts the ryanodine receptors thereby opening and allowing calcium to be released from the sarcoplasmic reticulum. Calcium will attach to troponin thereby displacing tropomyosin from the myosin-binding site (Ohtsuki \& Morimoto, 2008) thus allowing for the cross-bridge cycle. ATP binds to the myosin head eliciting a confirmational change in myosin thus decreasing its affinity for actin, thereby disassociating from actin allowing myosin to become cocked toward the sarcomere. ATP bound to myosin becomes hydrolyzed to ADP and Pi. While myosin is still in the cocked position, it will bind to a new site on actin creating a power stroke that pulls the actin filaments. ADP and Pi are released, and myosin returns to original state where it is bound to ATP (Fitts, 2008). The cross-bridge cycles are a series of events pulling thin filaments toward the center of the sarcomere each the action potential arrives at the neuromuscular junction. While the biochemistry of muscle contraction is well known, less is understood how the cachectic environment effects contractile properties and the exercise response. The next few sections will highlight what is known about skeletal muscle contractile properties in the 
cachectic environment and what current mechanisms explain exercise interventions as a potential therapeutic to offset wasting.

Cancer cachectic exhibits reduced volitional activity, whole body weakness, and fatigue in cancer patients and in pre-clinical models (Baltgalvis et al., 2010; Murphy et al., 2012; Toth et al., 2016). Furthermore, contractile decrements have also been reported (Christensen et al., 2014; Roberts, Frye, et al., 2013), and is highly important given that force production and fatigue are directly related to life quality in disease (al-Majid \& McCarthy, 2001; Barreiro \& Gea, 2015; Siegel, 1989). We and others have recently shown that cachectic skeletal muscle develops a slow-fatigable contractile phenotype during cancer cachexia progression (Murphy et al., 2012; Roberts, Frye, et al., 2013; VanderVeen et al., 2018). Furthermore, the regulation of muscle's contractile properties was strongly related to the muscle's inflammatory signaling highlighting the regulation of muscle contraction by inflammation during disease (VanderVeen et al., 2019). Interestingly, cachectic muscle exhibits increased SERCA1 and calsequesterin mRNA in cachectic skeletal muscle (VanderVeen et al., 2018), further supporting the transition to a more faster muscle phenotype (Khodabukus \& Baar, 2015). While fatigue and weakness are the most commonly reported consequences in cancer patients, we have only scratched the surface on trying to understand how this may occur (Chang et al., 2000). Interestingly, recent work has highlighted exercise's therapeutic role in improving muscle fatigability in patients and pre-clinical models (Kessels et al., 2018; Vanderveen et al., 2020). However, the mechanisms of how exercise can be a therapeutic have to yet to be fully understood.

Exercise has been hypothesized to be potential therapeutic option for cancer induced cachexia (Hardee et al., 2019). Voluntary wheel and treadmill running are the predominant methods of exercise used within the literature to offset cancer induced cachexia in pre-clinical models. Several indices of cachexia progression are attenuated in rodents given access to a running wheel by attenuating muscle mass loss and improving myofiber cross-sectional area (Coletti et al., 2016; Pigna et al., 2016). These studies highlight the improved ex vivo force production and reduced inflammatory environment likely attributing to the prevention of cachexia. In addition, treadmill training has also been shown to offset indices of cancer induced cachexia. Treadmill training was sufficient to decrease total polyp number (Mehl et al., 2005), likely through reducing immune infiltration, apoptosis, and beta-catenin signaling (Baltgalvis et al., 2010). Furthermore, exercise training prior to and during IL-6 overexpression prevented muscle mass loss, improved systemic glucose, and restored mTORC1 signaling (Lombardi et al., 2012; White et al., 2013). Interestingly, high intensity treadmill training improved survival rate and prevented muscle mass loss during cachexia progression (Jee et al., 2016) and recent work has supported that a potential exercise threshold might be required to reduce cachexia associated fatigue (Vanderveen et al., 2020). Alternatively, treadmill training was not sufficient to prevent muscle mass loss but was able to improve strength and muscle oxidative capacity (Pin et al., 2018). While there are discrepancies within the literature, it can be possibly attributed to the exercise modality, intensity, and basal capacity of the mouse given that cancer in tumor-bearing mice exhibit reduce volitional activity. However, there is sufficient evidence to suggest that exercise training prior to 
and during cancer progression can attenuate muscle mass and strength loss, which may be related to improved oxidative metabolism.

After an acute bout of exercise PGC-1 $\alpha$ is rapidly up-regulated leading to a subsequent increase in mitochondrial associated gene transcription and mitochondrial biogenesis (Baar et al., 2002; Pilegaard et al., 2003). Up-regulation of these genes persists for up to 4 hours before returning to baseline levels. Since the effects are short lived it shows the importance of regular physical activity to increase mitochondrial capacity. As well as increases in mitochondrial capacity, insulin sensitivity is increased immediately following an acute bout of exercise, in part due to the up regulation of genes regulating glycolysis and fatty acid oxidation and overall improvements of metabolic flexibility (Koves et al., 2013). For example, an acute bout of exercise insulin sensitivity is increased for at least 16 hours and lasting up to 48 hours (Bird \& Hawley, 2016). Furthermore, an acute bout of exercise is known to elicit a myokine "storm" known to aid in the beneficial effects of exercise and physical activity (Ost et al., 2016). Recent work has highlighted the potential therapeutic effect of myokines on cancer cachexia progression (Daou, 2020; Re Cecconi et al., 2019), however this has yet to be fully understood. Furthermore, an acute bout of exercise and exercise training are known regulators of muscle mitochondrial quality control (Yan et al., 2012). An acute bout of exercise (eccentric contractions) immediately induced AMPK and mTORC1 signaling in the cachectic environment (Hardee, Counts, et al., 2018). Interestingly, 3 hrs post contraction AMPK began to decrease while mTORC1 signaling remained elevated. Additionally, eccentric contraction training induced mitochondrial biogenesis and oxidative capacity further supporting the acute induction of AMPK on mitochondrial regulation. Although there is little research on the acute effects of exercise on mitochondrial dynamics during cancer cachexia, it can be hypothesized that exercise training would restore the altered expression of mitochondrial fusion proteins and decrease mitochondrial fission proteins.

Exercise training (repeated exercise bouts) is a known inducer of anabolic signaling through mTORC1 (Burd et al., 2009) and reduces chronic inflammation (Beavers et al., 2010). Furthermore, skeletal muscle contractions are sufficient to induce glucose uptake, fatty acid oxidation, mitochondrial biogenesis, and protein synthesis (Egan \& Zierath, 2013). The accumulation of lipids in the body can inhibit insulin sensitivity thus providing another benefit of regular exercise. Exercise training has a large impact on mitochondrial capacity in skeletal muscle. One of the main changes in skeletal muscle with exercise training is the increase in mitochondrial content and capacity (Holloszy \& Coyle, 1984). Repeated bouts of exercise show sustained increases in several mitochondrial proteins such as PGC-1 $\alpha$, mitochondrial transcription factor A (Tfam), and nuclear respiratory factors (NRF), thus allowing for mitochondrial biogenesis and increased mitochondrial function (Baar et al., 2002; Garnier et al., 2005; Hood et al., 2006). Furthermore, exercise is known to improve insulin sensitivity, and is often used to treat insulin resistance (Hawley, 2004). These improvements in insulin sensitivity are most likely derived from increases in skeletal muscle glucose transporter type 4; as GLUT4 can be stimulated both by insulin and by contraction. While muscle GLUT4 expression is unchanged in insulin resistant individual, these individual's show 
suppressed insulin signaling when stimulated (Handberg et al., 1990). The acute effects of exercise on glucose uptake occur in an insulin independent manner and rely mainly on the contraction stimulated increase in GLUT4 translocation (Brozinick et al., 1992). PI3K has been shown to be decreased in insulin resistant individuals (Luo et al., 2006) and is one reason for the decrease in insulin stimulated translocation of GLUT4 with insulin resistance. Exercise increases PI3K signaling potentially through increases in the insulin receptor substrate; however, the evidence is variable (Hawley \& Lessard, 2008).

Skeletal muscle mitochondrial function plays a pivotal role in muscle glucose uptake, and impairments in the oxidative capacity have been associated with insulin resistance (Lanza \& Nair, 2009). Alterations to mitochondrial content reduce the ability of muscle to efficiently oxidize fats, leading to disrupted metabolic flexibility (Goodpaster \& Sparks, 2017). The loss of muscle oxidative capacity during cancer cachexia corresponded with insulin resistance, despite this exercise training in the cachectic environment was sufficient to improve mitochondrial oxidative capacity (Lombardi et al., 2012). Exercise training allows for the ability to maintain mitochondria and ability to respond to stimulus that have capacity to impair metabolic flexibility. AMPK is a critical regulator for the exercise response in skeletal muscle. Exercise training increases protein levels of AMPK, but despite this exercise training induced an increase in muscle protein synthesis even in the cachectic environment. Exercise is also able to attenuate the chronic activation of AMPK that is seen with severe muscle wasting (Lombardi et al., 2012). The improvements in the regulation of AMPK activity may be due to the improvements in glucose uptake which could alleviate the energy stress that is placed on the muscle due to the cancer environment and metabolic inflexibility. More research needs to be done to examine the effects of exercise on muscle after the initiation of cachexia and if exercise can rescue mitochondrial dynamics and function. However, this has yet to be elucidated since most exercise interventions are used as a prevention.

To account for the baseline variability in treadmill and wheel exercise, electrical stimulation is another exercise modality used to elicit skeletal muscle contractions. Skeletal muscle's metabolic and growth response to contraction has been examined using electrical muscle stimulation (Baar \& Esser, 1999; Nader \& Esser, 2001; Puppa, Murphy, et al., 2014; Witkowski et al., 2010). In pre-clinical models, electrical stimulation is performed in an unconscious animal, which electrodes are used to stimulate a nerve at specific frequency. High-frequency electrical muscle stimulation (HFES) has examined muscle signaling associated with hypertrophy (Baar \& Esser, 1999; Hardee et al., 2016; Sato et al., 2019). In contrast, low-frequency electrical muscle stimulation (LFES) has been used to examine the acute and training adaptations to endurance like muscle contractions (Nader \& Esser, 2001; Puppa, Murphy, et al., 2014). Increased physical activity and repeated contractions impact skeletal muscle differently, for example high frequency stimulation $(100 \mathrm{~Hz}, 20$ minutes $)$ is a potent inducer of $\mathrm{p} 70 \mathrm{~S} 6 \mathrm{~K} 3 \mathrm{hrs}$ and $6 \mathrm{hrs}$ post in the TA, however no changes were observed in the soleus (Nader \& Esser, 2001). An acute exercise bout of low or high frequency stimulation has capacity to induce p70S6K, however HFES elicited a greater increased in p70S6K immediately post and 3 hour post stimulation compared to LFES (Tsutaki et al., 2013). Furthermore, p70S6Kwas induced $3 \mathrm{hrs}$ post LFES $(10 \mathrm{~Hz}, 30$ minutes), while an acute bout of running did not alter 
p70S6K in the soleus or TA thus concluding differentiational regulation of mTORC1 by exercise and muscle type. In human populations, a single bout of LFES improved glucose uptake (Hamada et al., 2003) and 4weeks of LFES induced markers of glucose metabolism and oxidative capacity (Theriault et al., 1994). Furthermore, 4wks of LFES improved fatigue resistance following spinal cord injury (Harridge et al., 2002). Taken together, LFES has therapeutic potential in understanding the molecular mechanisms of exercise in chronic disease.

Recent work has established a role for electrical stimulation to promote muscle hypertrophy and fiber type shifts in breast cancer patients, highlighting the clinical impactful role of electrical stimulation on muscle homeostasis in disease (Toth et al., 2020). Electrical stimulation has great therapeutic potential given the lack of volitional effort required and ability to provide a similar stimulus to non-tumor and tumor-bearing animals. We have shown that 2 wks. of eccentric contraction were sufficient to increase muscle mass in the cachectic environment, highlighting the ability of cachectic muscle to respond (Hardee et al., 2016). We have recently shown that repeated eccentric contractions are sufficient to induce anabolic signaling in the cachectic environment, coinciding with suppressed inflammatory signaling (Hardee et al., 2020). While eccentric contractions show promising results in preclinical models, the supraphysiological nature of eccentric contractions is not replicable of daily features. To this end, we completed an acute bout of low frequency stimulation in pre-clinical models of cancer induced cachexia. Interestingly, an acute bout of low frequency stimulation was capable of inducing rpS6, cytochrome $\mathrm{C}$, and PGC1-alpha in wild-type mice, however this was blocked in the cachectic mouse (Puppa, Murphy, et al., 2014). Taken together, repeated contractions were sufficient to improve oxidative capacity, implementing improved mitochondrial function, and highlights that cachectic muscle has the plasticity to respond to contraction stimulus. However, we have only began to understand the therapeutic potential of repeated muscle contractions on muscle oxidative capacity and significant barriers still exist in our understanding of repeated muscle contraction's effect on protein turnover in the cachectic environment. Furthermore, low frequency stimulation has been used for decades to understand the mechanical signaling of skeletal muscle (Scott et al., 1985 ) and is a plausible therapeutic option to combat disease. However, the effect of repeated low frequency stimulation on skeletal muscle homeostasis during cancer cachexia is unexplored.

\section{Conclusion}

Taken together, the current body of literature displays many gaps in understanding the regulation of skeletal muscle protein turnover during cancer cachexia progression by fasting and feeding and the direct role of muscle gp130 and AMPK. While we understand that cancer-induced cachexia disrupts skeletal muscle protein synthesis in the basal condition, we do not fully understand the regulation of feeding during cancer cachexia progression. A wide body of literature shows that inflammation is a key mediator of cancer-induced cachexia leading to disrupted mitochondrial dynamics, thereby activating AMPK. However, is it currently unknown if gp130's regulation of 
AMPK is linked to disrupted protein synthesis by feeding. Lastly, exercise interventions have shown promise in preventing cancer induced cachexia and is sufficient to improve mitochondrial function, induce anabolic signaling, and suppress inflammatory signaling. However, significant barriers still exist in our understanding if cachectic muscle has the capacity to respond to an acute bout of low frequency stimulation and feeding, and if it can improve muscle metabolic function and rescue the protein synthesis fasting. The current proposal aimed to understand if aberrant gp130 and AMPK signaling could alter the feeding and fasting regulation of muscle protein synthesis during cancer cachexia progression, and if muscle contraction could regulate this response. 


\section{CHAPTER 3. EXAMINATION OF WHETHER SKELETAL MUSCLE AMPK REGULATION BY FEEDING DISRUPTED EARLY IN CANCER CACHEXIA PROGRESSION IS RELATED TO EARLY DISRUPTIONS IN SYSTEMIC METABOLISM}

\section{Introduction}

Cancer-induced cachexia is defined as the unintentional loss of muscle mass with or without fat loss and is irreversible with nutritional support alone (Evans et al., 2008; Myers et al., 2019). Lung cancer is the second most common cancer type in the United States (Siegel et al., 2021), with 50\% of lung cancer patients developing cachexia (Jafri et al., 2015). The Lewis lung carcinoma (LLC) model is a widely used implantable syngeneic model to evaluate lung cancer progression (Kellar et al., 2015). Skeletal muscle mass maintenance during cachexia's progression has been linked to improved survival and quality of life (Bye et al., 2017). Cancer-induced disruption of muscle protein turnover is a negative consequence of the disease and a well-established driver of cachexia (White, Baynes, et al., 2011). There are currently no approved treatments to prevent or attenuate cachexia's progression, which is likely due to the complexity brought on by tumor heterogeneity and the cancer-induced systemic environment (Anderson et al., 2017). Accounting for skeletal muscle sensitivity to feeding could underlie some of these inconsistencies (Harfmann et al., 2015). Studies examining skeletal muscle protein synthesis during cancer cachexia have often either not controlled for feeding or examined after a brief fast. While we have made advances in understanding mechanisms of muscle wasting, we still lack sufficient information on how the progression of cachexia impacts the muscle's response to feeding as this has significant implications in understanding the potential therapeutic response.

Skeletal muscle's capacity for protein synthesis and degradation is highly sensitive to local and systemic stimuli that are integrated intracellularly through mTORC1 and AMPK signaling (Frost \& Lang, 2007). Oscillations in protein synthesis and breakdown occur throughout the day in response to feeding and fasting (Breen \& Phillips, 2011). Cancer-induced cachexia disrupts skeletal muscle protein turnover in the basal condition (decreasing protein synthesis and increasing protein degradation). In preclinical models, our laboratory has demonstrated altered protein turnover through the suppression of anabolic signaling via mTORC1 which coincided with chronic activation of AMPK (White, Baynes, et al., 2011). Interestingly, mTORC1 signaling exhibits diurnal fluctuations in response to changes throughout the day in feeding and activity (Counts et al., 2020). Furthermore, the cachectic environment disrupts these diurnal fluctuations, and suggest a link between disruptions in feeding and activity behaviors to the progression of cachexia and altered mTORC1 signaling (Counts et al., 2020). Feeding elicits an increase in circulating glucose to promote insulin release by the pancreas thereby translocating GLUT4 to the muscle's membrane to remove circulating glucose (Richter \& Hargreaves, 2013) and circulating insulin can also directly bind to IGF-1 resulting in a confirmational change to induce downstream phosphorylation of IRS/AKT/mTORC1 (Boucher et al., 2014). Feeding can directly target mTORC1 by 
increasing amino acid pool to promote protein synthesis (Liu \& Sabatini, 2020). Alternatively, fasting in pre-clinical models (24-48hrs) elicits an energy stress demand resulting in increased muscle AMPK signaling (Bujak et al., 2015). These findings have significant implications because more evidence is identifying a potential therapeutic window based on the time of day (Zhao et al., 2020) and nutritional state of the patient (Lammers et al., 2020). However, significant barriers still exist in our understanding of feeding's regulation of skeletal muscle protein synthesis during cancer cachexia.

AMPK is a regulatory point of interaction due to its numerous effects on muscle protein turnover by lowering ATP consumption of anabolic pathways and increasing catabolic pathways to produce ATP. AMPK is activated by energy stress when ATP bound to AMPK subunit is displaced with AMP. Throughout the day in healthy tissue, ATP:ADP ratios are in constant flux and exhibit a circadian rhythm with AMPK lowered after the dark cycle (Um et al., 2011). To disrupt these ratios, either there is an interference of ATP production or increased ATP consumption (Hardie, 2003). During periods of fasting, blood glucose levels begin to fall which interferes with ATP production, therefore, an increase of glucose results from activating glycogen use. Furthermore, during longer periods of fasting (starvation models $>16 \mathrm{hrs}$ ) after glycogen stores have been depleted, metabolism of triglycerides are used through beta oxidation and ketogenesis to produce ATP (Herzig \& Shaw, 2018). While these physiological changes to feeding and fasting occur throughout the day in healthy populations, significant barriers still exist in our understanding of how cachexia progression regulates AMPK signaling by feeding.

It is without question, that AMPK activity and associated signaling is necessary for maintaining metabolic flexibility and proper cell function (Fuchsberger et al., 2016; Jeon, 2016; Kjobsted et al., 2018; Steinberg \& Jorgensen, 2007). Additionally, it is necessary to highlight the adverse effects of AMPK loss in cardiac and skeletal muscle (Kjobsted et al., 2018; Viollet et al., 2009), which decreased functional outcomes in run time to fatigue, daily wheel distance, and cage activity (Maarbjerg et al., 2009; Morissette et al., 2014; Zwetsloot et al., 2008). AMPK $\alpha 2$ muscle-specific knockout (Miura et al., 2009) and AMPK $\alpha 2$ kinase dead in skeletal muscle (Moller et al., 2016) decreased running capacity, AMPK $\beta 1 / \beta 2$ muscle loss decreased muscle fiber size and capillary density (Thomas et al., 2014), and AMPK $\gamma 3$ loss decreased glycogen content (Canto et al., 2010). These studies highlight the importance of AMPK in skeletal muscle under normal physiological conditions. Under conditions of chronic disease, the effect of AMPK loss is less understood which is partially due to the desire to increase AMPK activity acutely either pharmacologically or by an acute bout of exercise (Jeon, 2016). However, it is important to consider disease conditions such as cancer cachexia which exhibit chronically elevated AMPK signaling (Fix et al., 2021; White et al., 2013), which is different than the acute activation elicited by drugs or exercise. It is ill-advised to suggest the therapeutic potential to knockout AMPK to treat cachexia, however the loss of AMPK in skeletal muscle alone allows us to determine the importance of AMPK signaling in response to an acute stress (12hr fast) and an anabolic stimulus in hopes to understand AMPK's regulation. Additionally, it is necessary to mention there are limited number of studies that examined the effect of whole-body AMPK activation throughout 
cachexia progression. Briefly, AMPK activators, such as AICAR and Metformin administered before cachexia initiation, have been examined with some preclinical models of cancer cachexia. AICAR administration prevented muscle mass loss in tumorbearing mice (Hall et al., 2018; Pigna et al., 2016) and lower E3 ligase gene expression and autophagy indices (Pigna et al., 2016), however AICAR inhibited tumor growth as well. Metformin can also prevent indices of cachexia (Hall et al., 2018; Oliveira \& Gomes-Marcondes, 2016). Despite these facts, there is a limited understanding of AMPK's regulation in differential nutrient conditions in skeletal muscle during cancer cachexia progression.

Taken together, cachexia suppresses muscle protein synthesis which coincided with an increase in muscle AMPK signaling in the basal condition. Therefore, we extended previous studies by examining if tumor-bearing mice have the capacity for feeding to increase muscle protein synthesis, and if the fasting suppression of protein synthesis is regulated by AMPK in tumor-bearing mice. Therefore, the purpose of this study was to determine if skeletal muscle AMPK regulation by feeding is disrupted early in cancer cachexia progression and is related to early disruptions in systemic metabolism. We hypothesized that feeding could reduce AMPK signaling and cancer cachexia progression would inhibit this response. We also hypothesized that disrupted AMPK signaling would be associated to early disruptions in systemic metabolism.

\section{Methods}

\section{Effect of feeding in tumor-bearing mice}

Male C57BL/6J (B6; N=42) mice were bred at the University of Tennessee Health Science Center Animal Resource Facility. B6 mice were initially purchased from Jackson Labs (Bar Harbor, ME, USA). Mice were kept on a 12:12h light/dark cycle beginning at 6:00AM and were given rodent chow ad libitum (Harlan Teklad Rodent Diet, \#8604, Harlan, Indianapolis, IN, USA). All mice were fasted for $12 \mathrm{hrs}$ during the dark cycle. At the start of the light cycle, mice were either given ad libitum access to a food pellet (fed) or sacrificed at the end of fast (fast).

\section{Lewis lung carcinoma cell inoculation}

Between 11-12 weeks of age, B6 mice were injected with either phosphate buffered saline (PBS) or $1 \times 10^{6}$ Lewis lung carcinoma (LLC) cells (ATCC CRL-1642) subcutaneously in the right flank under anesthesia for less than 3 minutes (Puppa, Gao, et al., 2014). A total of 26 mice were injected with LLC cells and 16 mice were injected with PBS. Ninety-two percent of the LLC injected mice reached the studies endpoint. A mouse reached the studies endpoint if the following criteria were met (1) mouse reached 30 days post tumor inoculation, (2) $>20 \%$ body weight loss from day 10 after day 25 , or (3) had a tumor $>3 \mathrm{~cm}$ in width or length after day 25 , or (4) the tumor was close to 
breaking through the skin (ulcerated) after day 25. Once an endpoint was achieved, the mouse was prepared for tissue collection and euthanized within $24 \mathrm{hrs}$. At day 21, 1 mouse had an ulcerated tumor and was not included in the analysis and 1 mouse died unexpectedly at day 24 . To be included in the study, mice needed to achieve at least 25 days post tumor inoculation. Therefore, a total of 24 male LLC injected mice were used in these experiments. Tumor volume and body weight were measured every 5 days to calculate tumor growth. Tumor volume was calculated by the same investigator and caliper using the following equation: $1 / 2$ (width ${ }^{2}$ xlength) (Jensen et al., 2008).

\section{Muscle-specific AMPK loss in tumor-bearing mice}

To examine the regulation of AMPK loss in tumor-bearing mice, AMPKa ${ }^{1} a^{2}$ knockout mice and floxed controls were generated (see next section for details). Mice were kept on a 12:12h light/dark cycle beginning at 6:00AM and were given rodent chow ad libitum (Harlan Teklad Rodent Diet, \#8604, Harlan, Indianapolis, IN, USA). Between 17-18wks of age, male AMPKa $\mathrm{a}^{2}$ knockout or floxed controls either injected with PBS or LLC cells were sacrificed. All mice were fasted for $12 \mathrm{hrs}$ during the dark cycle. At the start of the light cycle, mice were either given ad libitum access to a food pellet (fed) or sacrificed at the end of fast (fast).

\section{Generation of AMPKa ${ }^{1} \mathbf{a}^{2}$ skeletal muscle deletion}

To generate a skeletal muscle-specific knockout of AMPKa ${ }^{1} \mathrm{a}^{2}$, mice that contained individually floxed alleles for $\mathrm{AMPKa}^{1}$ and $\mathrm{AMPKa}{ }^{2}$ were a kind gift from Dr. Hoh-Jin Koh at the University of South Carolina. Utilizing the skeletal actin (HSA) promoter-driven expression of a Cre recombinase flanked by mutated estrogen receptors (HSA- MCM) (McCarthy et al., 2012). Tamoxifen inducible Mer Cre Mer driven by a human skeletal actin promoter (HSA) mouse were purchased from Jackson Laboratories (Bar Harbor, ME, USA). Male tamoxifen-inducible skeletal muscle AMPK $\alpha^{1} \alpha^{2}$ (KO) knockout mice and floxed (Flox) controls were produced. This genetic approach provides viable, healthy mice and has been successfully used by others to study muscle-specific inducible loss of AMPK in skeletal muscle (Hingst et al., 2020; Lantier et al., 2020). At approximately 10-11 weeks of age, all AMPK KO and floxed control mice received tamoxifen injection (I.P. 2mg) daily for 5 consecutive days (Harfmann et al., 2016), and then underwent a 2-week washout period before PBS or LLC injections. Finally, the AMPK $\alpha^{1} \alpha^{2}$ HSA Cre (AMPK KO) mice or AMPK $\alpha^{1} \alpha^{2}$ (floxed control) mice were injected with PBS or LLC, following a similar study design as experiment 1.

\section{Lewis lung carcinoma cell inoculation in muscle-specific AMPK mice}

Between 13-14 weeks of age, AMPK mice were injected with either phosphate buffered saline (PBS) or $1 \times 10^{6}$ Lewis lung carcinoma (LLC) cells subcutaneously in the right flank under anesthesia for less than 3 minutes (Puppa, Gao, et al., 2014). A total of 
38 mice were injected with LLC cells and 32 mice were injected with PBS. Ninety-two percent of the LLC injected mice reached the studies endpoint. A mouse reached the studies endpoint if the following criteria were met (1) mouse reached 30 days post tumor inoculation, (2) $>20 \%$ body weight loss from day 10 after day 25 , or (3) had a tumor $>3 \mathrm{~cm}$ in width or length after day 25 . Once an endpoint was achieved, the mouse was prepared for tissue collection and euthanized within $24 \mathrm{hrs}$. At day 22-23, 3 mice had ulcerated tumors and was not included in the analysis. To be included in the study, mice needed to achieve at least 25 days post tumor inoculation. Therefore, a total of 35 male LLC injected mice were used in these experiments. Tumor volume and body weight were measured every 5 days to calculate tumor growth. Tumor volume was calculated by the same investigator and caliper using the following equation: $1 / 2$ (width ${ }^{2}$ xlength) (Jensen et al., 2008).

\section{Tissue collection}

Mice were anesthetized and $\sim 500 \mathrm{ul}$ of retro-orbital blood was collected. Following eye bleeds, under anesthesia mice underwent cervical dislocation and muscles were immediately excised within 4 minutes. A second method of euthanasia was heart removal, then organs were collected. Hindlimb muscles and organs (eWAT, liver, stomach, heart, kidney, and spleen) were rapidly excised, cleared for excessive connective tissue, weighed, and snap frozen in liquid nitrogen.

\section{Plasma interleukin-6}

Immediately prior to sacrifice, blood was collected via retro-orbital sinus with heparinized capillary tubes, placed on ice, and centrifuged $\left(10,000 \mathrm{xg}\right.$ for $10 \mathrm{~min}$ at $\left.4^{\circ} \mathrm{C}\right)$. The supernatant was removed, and plasma IL-6 concentrations were determined using an enzyme-linked immunosorbent assay kit according to the manufacturer's instructions (Catalog \#KMC0061, ThermoFisher Scientific, Waltham, MA).

\section{Blood glucose and plasma insulin}

Immediately prior to ad libitum access to a food pellet, mice had their tail snipped. The initial blood was wiped with a Kim wipe and blood glucose was determined using a standard and readily available glucometer (Contour Next, Parsippany, NJ). Blood was collected with a heparinized capillary tube, placed on ice, and centrifuged $(10,000 \mathrm{x}$ $\mathrm{g}$ for $10 \mathrm{~min}$ at $4^{\circ} \mathrm{C}$ ). The supernatant was removed, and plasma insulin concentrations were determined using a two-site enzyme immunoassay assay kit according to the manufacturer's instructions (Catalog \#10-1247, Mercodia, Uppsala, Sweden). Blood glucose and plasma insulin measurements were taken again 1-hr after access to the food pellet but prior to anesthesia at sacrifice. 


\section{Protein synthesis measurement}

The Surface Sensing of Translation (SUnSET) technique was used to determine estimated muscle protein synthesis rates, as previously described (Goodman et al., 2011) (Hardee, Counts, et al., 2018). Briefly, puromycin (EMD Millipore, catalog no. MABE343) was dissolved in sterile saline and delivered by intraperitoneal injection $(0.04$ $\mu \mathrm{mol} / \mathrm{g}$ body wt.) $30 \mathrm{~min}$ before euthanasia. Puromycin incorporation into newly synthesized peptides, reflecting estimated global protein synthesis rates, and was analyzed by Western blot.

\section{Western blot}

Western blot analysis was performed as previously described (Hardee et al., 2014) and extensive methodology can be found in extended methods section. Briefly, frozen red and white gastrocnemius muscle was homogenized in lysis buffer and protein concentration was determined by the Bradford method. Crude gastrocnemius muscle homogenates were fractionated on $8-12 \%$ SDS-polyacrylamide gels and transferred to PVDF membranes. Membranes were stained with Ponceau red to verify equal loading and transfer. Membranes were then blocked at room temperature for $1 \mathrm{hr}$. in 5\% non-fat milk or 5\% BSA-Tris-buffered saline with 0.1\% Tween-20 (TBST). Commercially available phosphorylated and total protein primary antibodies for rpS6, AKT, AMPK, ACC, FOXO3a, ULK1, and DRP1 were raised in rabbit. RpS6, AKT, AMPK, ACC, FOXO3a, ULK1, and DRP1 phosphorylation antibodies were expressed relative to total protein on the same gel and quantified as phosphorylation to total ratio. Commercially available total protein primary antibodies for MuRF-1, Atrogin-1, PGC1 $\alpha$, OPA1, and FIS1 were raised in rabbit. Puromycin incorporation was purchased from Millipore and raised in mouse. Briefly, primary antibodies were incubated overnight in 5\% TBST milk or 5\% TBST BSA. Membranes were then incubated in 5\% milk-TBST containing antirabbit or anti-mouse IgG horseradish-peroxidase conjugated secondary antibody purchased from cell signaling for $1 \mathrm{~h}$ at room temperature. Enhanced chemiluminescence (ECL) (GE Healthcare Life Sciences, Piscataway, NJ) was used to visualize the antibodyantigen interactions. Immunoblot images were collected using a digital imager (Invitrogen iBright, Waltham, MA) and quantified by densitometry using imaging software (Image J; NIH). Please see end of document for details of fcatalogue numbers, companies, dilutions, incubation durations, and secondary substrate (Figure A-1).

\section{Body composition and indirect calorimetry}

Fifteen days post tumor inoculation, a subset of mice (PBS $n=5$, LLC $n=6$ ) were individually housed and placed in the Comprehensive Laboratory Animal Monitoring System (CLAMs) cages for 5-consecutive days (Stephenson et al., 2016). To allow for acclimatization, the first $24 \mathrm{hrs}$ were removed from the analysis and the average of days 2 , 3 , and 4 were used for analysis. Mice were removed from the cages on day 5. Data are presented as daily average, and the average of each light and dark cycle. In mice, light 
cycle refers to the time when mice are resting (rest phase) and consuming little to no

food. While the dark cycle, refers to the active phase when they consume most of their daily food consumption (Counts et al., 2020; Jensen et al., 2013). Prior to individual housing, mice were weighed between 7:00-9:00 AM and body composition was determined in conscious mice by magnetic resonance (EchoMRI 1100, EchoMRI, Houston, TX). Using the CLAMs system (Columbus Instruments, Columbus, OH) physical activity, oxygen consumption (VO2), carbon dioxide production (VCO2), and respiratory exchange ratio (RER; VCO2/VO2) were determined. Physical activity was quantified as the 3-day average of the hourly sum of XY beam breaks. The temperature was maintained at $22^{\circ} \mathrm{C}$ throughout the experiment. The Lusk equation in Oxymax software (Columbus Instruments, Columbus, $\mathrm{OH}$ ) was used to determine energy expenditure. Carbohydrate and lipid oxidation were based on published equations that assume negligible protein oxidation (Peronnet \& Massicotte, 1991). Before use in the equation, $\mathrm{VO} 2$ and $\mathrm{VCO} 2$ were converted from $\mathrm{ml} / \mathrm{kg}$ lean mass $/ \mathrm{hr}$ to $\mathrm{ml} / \mathrm{min}$. Equations are as follows: carbohydrate oxidation $(\mathrm{g} / \mathrm{min}):\left(4.585^{*} \mathrm{VCO} 2\right)-(3.226 * \mathrm{VO} 2)$ and lipid oxidation $(\mathrm{g} / \mathrm{min})(1.695 * \mathrm{VO} 2)-(1.701 * \mathrm{VCO} 2)$, and negative values indicate unusable time points which were removed from the analysis.

\section{Glucose tolerance test}

After a $6 \mathrm{hr}$ fast, mice received a single glucose bolus ( $2 \mathrm{~g} / \mathrm{kg}$ lean mass) via intraperitoneal injection, and blood glucose concentrations were monitored at 15 or 30min intervals throughout the subsequent $120 \mathrm{~min}$. Aliquots of blood were collected at pre, 15-, 30-, 60-, and 120-min post injection.

\section{Statistical analysis}

Pre-planned unpaired T-Test were used to compare PBS and LLC mice. Preplanned unpaired T-Test were used to compare the feeding response and knockout signaling in PBS and LLC mice. Two-way ANOVA's (Tumor x Feeding) were used and Tukey's post-hoc analysis was used when appropriate. Pearson spearman correlations were used to associate AMPK signaling and signaling with cachexia progression, systemic metabolism, and physical activity. Prism 8 (GraphPad) was used for statistical analysis. All results are reported as means \pm standard error of mean (SEM). Statistical analyses were performed using GraphPad (Prism 8 for Mac OS X, La Jolla, Ca). Level of significance for all measures was set at $\mathrm{p} \leq 0.05$.

\section{Study approval}

All experiments were approved by the University of Tennessee Health Science Center's Institutional Animal Care and Use Committee (protocol \#19.001). 


\section{Results}

\section{LLC characterization}

Between 11-12 weeks of age C57BL/6J (B6) mice were injected with PBS C cells and were then sacrificed 25-30 post injection. There were no differences in age or study duration between PBS ( $=16)$ and LLC $(\mathrm{N}=24)$ mice (Table 3-1). The study was initiated at approximately 12 weeks of age and completed at approximately 15 to 16 weeks of age. PBS and LLC mice bodyweights were similar before tumor inoculation $(p=0.515)$ and 10 days post tumor inoculation $(p=0.445)$. While there were no differences in absolute body weight at the end of the study $(\mathrm{p}=0.397)$, after accounting for tumor mass, LLC mice had decreased body weight compared to PBS mice $(p=0.001)$ (Figure 3-1). LLC mice had a greater body weight change from day 10 when accounting for tumor mass (-5.7\%) compared to PBS mice (0.4\%) (Figure 3-1G). Body weight change from day 10 was chosen because the tumor is initially palpable but prior to the rapid tumor growth phase (days 15-25). LLC mice had decreased gastrocnemius muscle mass (Figure 3-1H), total hindlimb muscle mass $(\mathrm{p}=0.008)$ (Table 3-1), and eWAT mass (Figure 3-1) compared to PBS mice. LLC mice had increased spleen mass and plasma IL-6 (Table 3-1) compared to PBS mice. Tumor volume and tumor mass were used to validate tumor development. Tumor volume was measured every 5 days throughout the study and was measurable 10 days post tumor inoculation (Figure 3-1). Utilizing the LLC model's rapid tumor growth phase, we calculated the slope of tumor growth from days 15-25. Tumor growth rate ranged from 0.090 to $0.383 \mathrm{~cm}^{3} / 5$ days (Figure 3-1C). Tumor mass ranged from 0.745 to 5.15 grams (Figure 3-1). Tumor growth rate from days $15-25$ and tumor mass were not associated $(r=0.387, \mathrm{p}=0.062)$.

We then compared common proteins elevated in cachectic skeletal muscle: STAT3, P65, and Atrogin-1. LLC mice had elevated STAT3 0.9-fold, P65 0.6-fold, and Atrogin-1 0.7fold compared to PBS mice (Figure 3-1J). Taken together, these results provide evidence of pre-cachectic tumor-bearing mice (Figure B-1). To examine the effect of feeding on systemic metabolism and muscle signaling, we first need to examine if there are differences in animal characteristics between the fast and fed condition in both PBS and LLC treated mice (Table 3-2). In PBS mice, there were no differences in body weight throughout the study, gastrocnemius muscle mass, eWAT mass, or tibia length between the fast and fed condition. Additionally, in LLC mice, there were no differences in body weight throughout the study, gastrocnemius muscle mass, eWAT mass, or tibia length between the fast and fed condition. Importantly, there were no. differences in tumor growth, tumor growth rate, nor tumor mass in LLC mice in the fast and fed condition Given that there were no differences between the fast and fed condition of PBS and LLC mice, we can compare the whole-body and muscle response to feeding between groups of PBS and LLC mice, we can compare the whole-body and muscle response to feeding between groups. 
Table 3-1. Animal characteristics of LLC tumor-bearing male mice

\begin{tabular}{|c|c|c|c|}
\hline Characteristics & $\begin{array}{c}\text { PBS } \\
\mathrm{N}=16\end{array}$ & $\begin{array}{c}\text { LLC } \\
\mathbf{N}=\mathbf{2 4}\end{array}$ & p-Value \\
\hline BW Pre-treatment (g) & $25.5(0.6)$ & $24.9(0.3)$ & 0.362 \\
\hline BW d10 (g) & $26.5(0.5)^{*}$ & $25.7(0.3)^{*}$ & 0.180 \\
\hline BW End of Study (g) & $26.6(0.4)$ & $27.0(0.3)$ & 0.370 \\
\hline BW End of Study & $26.6(0.4) *$ & $24.2(0.4) \#$ & $<0.001$ \\
\hline Tumor Mass (g) & & $2.8(0.3)$ & \\
\hline BW $\Delta$ During Treatment (\%) & $4.6(1.4)$ & $2.6(1.6)$ & 0.003 \\
\hline $\mathrm{BW} \Delta \mathrm{d} 10(\%)$ & $0.4(0.8)$ & $-5.7(1.3)$ & 0.001 \\
\hline Tumor Volume d15 $\left(\mathrm{cm}^{3}\right)$ & - & $0.42(0.09)$ & \\
\hline Plasma IL-6 (pg/ml) & $3.9(0.0)$ & $15.3(2.2)$ & 0.001 \\
\hline HMM (mg) & $192(4)$ & $175(3)$ & 0.002 \\
\hline eWAT (mg) & $328(30)$ & $240(16)$ & 0.008 \\
\hline Testes (mg) & $199(4)$ & $203(3)$ & 0.386 \\
\hline Heart (mg) & $153(5)$ & $137(4)$ & 0.023 \\
\hline Spleen (mg & $73(6)$ & $220(27)$ & $<0.001$ \\
\hline Tibia Length $(\mathrm{mm})$ & $16.8(0.1)$ & $16.8(0.1)$ & 0.810 \\
\hline
\end{tabular}

Notes: Data are presented as mean \pm SEM. Abbreviations: BW: body weight, mg: milligrams, eWAT: epididymal white adipose tissue, PBS: phosphate buffered saline, LLC: Lewis lung carcinoma, mm: millimeter, g: grams, HMM: hindlimb muscle mass. Unpaired T-Test were used to compare differences between PBS and LLC mice. Oneway repeated measures ANOVA was used to compare within PBS and LLC mice body weight over the course of the study (BW End of study is not included in analysis) * Different from Pre within treatment group, \# Different from BW d10 within treatment group. One-way repeated measures ANOVA was used to compare tumor volume over the course of the study. * Different from d15 within treatment group, \# Different from d20 within treatment group Statistically significant $p<0.05$. 
A.

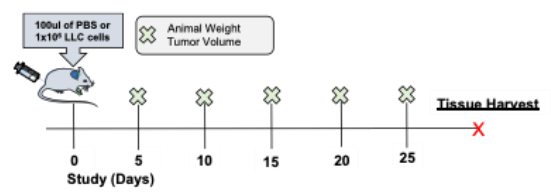

B.

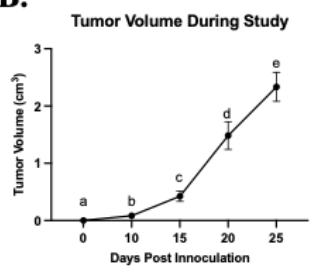

E.
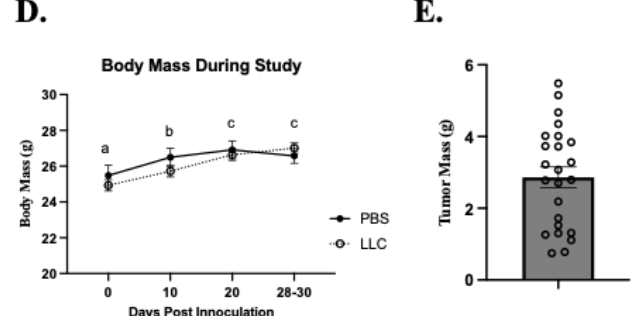

H.

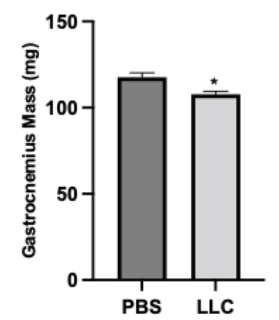

I.

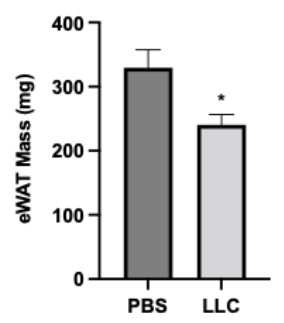

C.

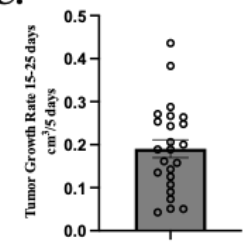

G.

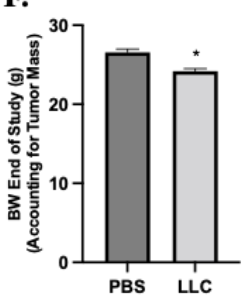

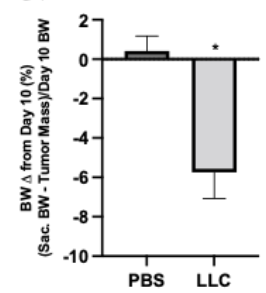

Figure 3-1. LLC tumor-bearing male mice exhibit several indices of cachexia and increased skeletal muscle inflammatory signaling

Notes: Data are presented as mean \pm SEM. A. Study Design. Male C57B1/6J mice were injected with PBS or LLC cells between 10-11 wks. of age. Tumor volume and body weight were taken every 5 days until sacrifice (28-30 days post tumor inoculation). B.Tumor volume during the study in LLC male mice. C. Tumor growth rate from days 1525. D. Body mass during the study in LLC and PBS male mice. E. Tumor Mass at sacrifice. F. Body weight at the end of study accounting for tumor mass. G. Body weight change from Day 10 post tumor inoculation. Body weight is calculated as the sacrifice body weight minus the tumor mass divided by day 10 body weight. H. Gastrocnemius muscle mass at sacrifice. I. eWAT mass at sacrifice. J. Cachectic skeletal muscle signaling in mixed gastrocnemius muscle. Abbreviations: BW: body weight, mg: milligrams, PBS: phosphate buffered saline, LLC: Lewis lung carcinoma, g: grams. Unpaired t-test was used to compare PBS to LLC. * Different between groups. Pearson Correlation Coefficient was used for correlations. Statistical significance was set a $\mathrm{p}<0.05$. 
Table 3-2. Animal characteristics of PBS and LLC male mice in the fast and fed condition

\begin{tabular}{|c|c|c|c|c|}
\hline \multirow[b]{2}{*}{ Characteristics } & \multicolumn{2}{|c|}{ PBS } & \multicolumn{2}{|c|}{ LLC } \\
\hline & $\begin{array}{l}\text { Fast } \\
N=7\end{array}$ & $\begin{array}{l}\text { Fed } \\
N=9\end{array}$ & $\begin{array}{c}\text { Fast } \\
N=10\end{array}$ & $\begin{array}{c}\text { Fed } \\
N=14\end{array}$ \\
\hline BW Pre-treatment $(\mathrm{g})$ & $25.3(1.0)$ & $25.7(0.6)$ & $24.5(0.6)$ & $25.3(0.4)$ \\
\hline BW d10 (g) & $26.5(0.9)^{\mathrm{a}}$ & $26.5(0.6)^{a}$ & $26.1(0.5)^{\mathrm{a}}$ & $25.4(0.4)$ \\
\hline BW End of Study (g) & $26.5(0.8)$ & $27.1(0.6)$ & $26.5(0.5)$ & $27.2(0.4)$ \\
\hline BW End of Study & 26.4. (0.8) & $26.7(0.5)$ & $24.5(0.5)^{\mathrm{b}} \$$ & $24.2(0.5)^{b} \$$ \\
\hline Tumor Mass (g) & - & - & $2.3(0.4)$ & $3.1(0.4)$ \\
\hline BW $\Delta$ During Treatment (\%) & $5.0(2.4)$ & $4.3(1.7)$ & $-0.7(2.9)^{\wedge}$ & $-4.4(1.9)^{\wedge}$ \\
\hline $\mathrm{BW} \Delta \mathrm{d} 10(\%)$ & $-0.3(1.1)$ & $0.9(1.1)$ & $-5.8(1.6)^{\wedge}$ & $-4.1(1.9)^{\wedge}$ \\
\hline Tumor Volume d15 $\left(\mathrm{cm}^{3}\right)$ & - & - & $0.17(0.05)$ & $0.60(0.03) *$ \\
\hline Tumor Volume d20 $\left(\mathrm{cm}^{3}\right)$ & - & - & $1.19(0.27)^{\mathrm{a}}$ & $1.69(0.36)^{\mathrm{a}}$ \\
\hline Tumor Volume d25 $\left(\mathrm{cm}^{3}\right)$ & - & - & $2.00(0.35)^{\mathrm{a}, \mathrm{b}}$ & $2.57(0.35)^{\mathrm{a}, \mathrm{b}}$ \\
\hline Tumor Slope Rate d15-25 & - & - & $0.182(0.034)$ & $0.197(0.027)$ \\
\hline Plasma IL-6 (pg/ml) & $3.9(0.0)$ & $3.9(0.0)$ & $10.6(2.6)^{\wedge}$ & $18.6(3.0)^{\wedge}$ \\
\hline HMM (mg) & $193(8)$ & $191(5)$ & $181(5)^{\wedge}$ & $171(3)^{\wedge}$ \\
\hline eWAT (mg) & $365(45)$ & $310(35)$ & $256(23)^{\wedge}$ & $229(22)^{\wedge}$ \\
\hline Testes (mg) & $201(7)$ & $197(5)$ & $204(5)$ & $202(3)$ \\
\hline Heart (mg) & $151(9)$ & $154(7)$ & $147(7)^{\wedge}$ & $130(3)^{\wedge}$ \\
\hline Spleen (mg) & $86(8)$ & $62(5)$ & $248(62)^{\wedge}$ & $200(14)^{\wedge}$ \\
\hline Tibia Length (mm) & $16.9(0.1)$ & $16.8(0.1)$ & $16.9(0.1)$ & $16.8(0.1)$ \\
\hline
\end{tabular}

Notes: Data are presented as mean \pm SEM. Abbreviations: BW: body weight, $\mathrm{mg}$ : milligrams, eWAT: epididymal white adipose tissue, PBS: Phosphate buffered saline, LLC: Lewis lung carcinoma, mm: millimeter, g: grams, ME: Main Effect, HMM: Hindlimb muscle mass. One-way repeated measures ANOVA was used to compare within LLC fast and fed mice body weight over the course of the study (BW End of study is not included in analysis/ BW end of study accounting for tumor mass is included).

$a=$ Different from Pre within treatment group, $b=$ Different from BW d10 within treatment group. One-way repeated measures ANOVA was used to compare tumor volume over the course of the study. $a=$ Different from $d 15$ within treatment group and $b=$ Different from $\mathrm{d} 20$ within treatment group. Statistically significant $p<0.05$. Two-way ANOVA was used to compare PBS and LLC in the Fast and Fed condition. \# Main Effect of Feed. \$Main effect of LLC. Unpaired T-test were used to compare tumor growth and mass in the fed and fast condition of LLC mice. * Different from LLC fast. 


\section{Whole-body response to feeding during cancer cachexia progression}

After 4 weeks of tumor inoculation, mice were fasted for 12-hrs during the dark cycle, then sacrificed (fast) or given access to a food pellet for 1-hr ad libitum (fed) (Figure 3-2). There were no differences in pellet consumption in fed PBS and LLC mice (Figure 3-2). There was an interaction for stomach mass and stomach content to increase in both PBS and LLC mice in the fed condition (Table 3-3). Interestingly, PBS mice increased stomach mass to a greater extent than LLC mice (Table 3-3). There were no differences in empty stomach mass. There was a main effect for blood glucose to increase by feeding in both PBS and LLC mice (Figure 3-2). We completed a pre-planned t-test within treatment groups (PBS or LLC) to validate the insulin response by feeding. We report that plasma insulin increased in the PBS fed $(p=0.002)$ compared to PBS fast mice. Plasma insulin was increased in the LLC fed $(\mathrm{p}<0.001)$ compared to LLC fast mice. When comparing PBS and LLC in the fast and fed condition, there was an interaction for plasma insulin to increase in the PBS fed mice, however the insulin induction by feeding was not present in LLC mice (Figure 3-2).

We next examined the feeding response in skeletal muscle. We first validated direct downstream insulin signaling, phospho-AKT T308 (Figure B-2). There was a main effect for feeding to increase AKT 3.5-fold compared to fast, and a main effect for PBS mice to have increased AKT 1.5-fold compared to LLC (Figure 3-2). Next, we examined downstream mTORC1 signaling, rpS6. There was a main effect for feeding to increase rps6 4.2-fold compared to fast, and a main effect for PBS mice to have increased rpS6 1.3-fold compared to LLC mice (Figure 3-2). Lastly, we examined the effect of feeding on skeletal muscle protein synthesis. There was a main effect for feeding to increase puromycin incorporation 2-fold compared to fasting, and a main effect for PBS mice to have increased puromycin incorporation 1.7-fold compared to LLC mice (Figure 3-2). Taken together these results validate the anabolic response to feeding in PBS mice (non-tumor-bearing mice) by an increase in blood glucose, plasma insulin, muscle AKT/mTORC1 signaling, and muscle protein synthesis. Additionally, we report that tumor-bearing mice can elicit an increase blood glucose and plasma insulin in response to feeding. Importantly, LLC tumor-bearing mice do have the ability to increase in $\mathrm{AKT} / \mathrm{mTORC} 1$ and protein synthesis following feeding, however LLC mice have overall lower anabolic muscle signaling independent of fast or fed condition.

\section{Muscle Akt/mTORC1 and protein synthesis association to cachexia progression}

We next asked the question if anabolic signaling in the fasting condition was associated with cachexia progression (common indices of cachexia: gastrocnemius mass, eWAT, and body weight change). AKT in LLC fast mice was not associated with gastrocnemius muscle mass $(r=-0.479, \mathrm{p}=0.230)$, eWAT mass $(\mathrm{r}=-0.408, \mathrm{p}=0.315)$ nor body weight change from day $10(\mathrm{r}=0.554, \mathrm{p}=0.154)$. RpS6 in LLC fast mice was not associated with gastrocnemius muscle mass $(\mathrm{r}=0.479, \mathrm{p}=0.230)$, eWAT mass $(\mathrm{r}=-0.408$, $\mathrm{p}=0.315)$ nor body weight change from day $10(\mathrm{r}=0.554, \mathrm{p}=0.154)$. Lastly, puromycin 
A.
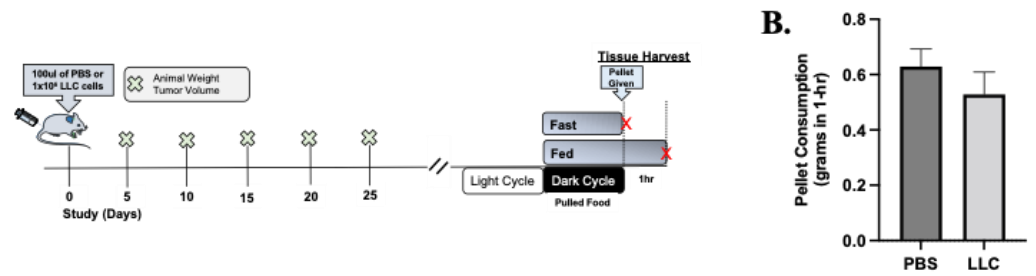

C.

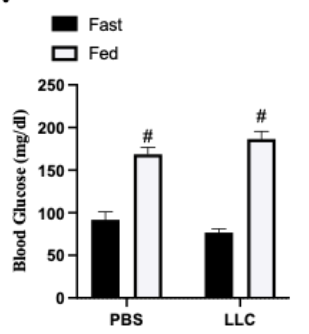

F.

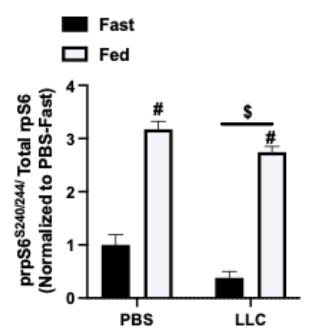

D.

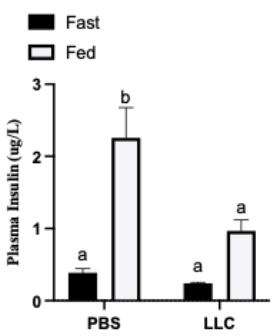

G.

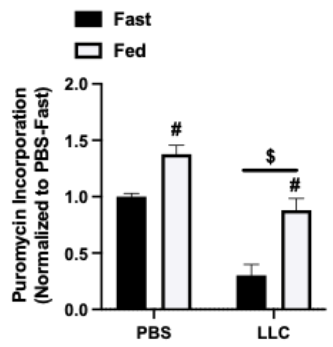

E.

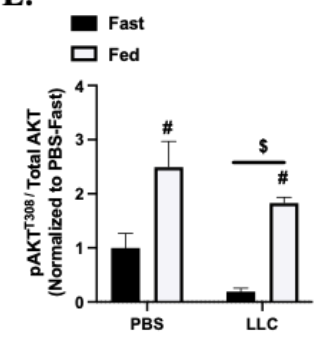

H.

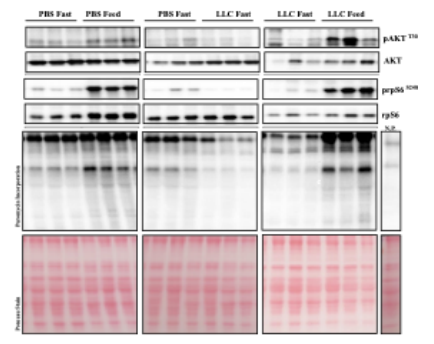

Figure 3-2. LLC tumor-bearing mice exhibit whole-body and muscle-specific anabolic response to $1-\mathrm{hr}$ ad libitum feeding

Notes: Data are presented as mean \pm SEM. A. Study Design. Male C57Bl/6J mice were injected with PBS or LLC cells between 10-11 wks. of age. Tumor volume and body weight were taken every 5 days until sacrifice (28-30 days post tumor inoculation). Pellet consumption in Fed PBS and LLC mice. C. Blood glucose at Sacrifice. D. Plasma Insulin at Sacrifice. E. Gastrocnemius muscle phoshpo-AKTT308 to total protein expression ratio. F. Gastrocnemius muscle phospho-rpS6S240/244 to total protein expression ratio. G. Gastrocnemius muscle puromycin incorporation protein expression. H. Representative western blots. Abbreviations: BW: body weight, mg: milligrams, PBS: phosphate buffered saline, LLC: Lewis lung carcinoma, g: grams. Unpaired t-test was used to compare PBS to LLC. * Different between groups. Pearson Correlation Coefficient was used for correlations. Statistical significance was set a $p<0.05$. N=7 PBS fast, 9 PBS Fed, 12 LLC. \#Main effect of Fed, \$ Main effect of LLC. 
Table 3-3. Whole body response to feeding in PBS and LLC male mice

\begin{tabular}{|c|c|c|c|c|}
\hline \multirow[b]{2}{*}{ Outcomes } & \multicolumn{2}{|c|}{ PBS } & \multicolumn{2}{|c|}{ LLC } \\
\hline & $\begin{array}{l}\text { Fast } \\
N=7\end{array}$ & $\begin{array}{l}\text { Fed } \\
N=9\end{array}$ & $\begin{array}{c}\text { Fast } \\
N=10\end{array}$ & $\begin{array}{c}\text { Fed } \\
N=14\end{array}$ \\
\hline Pellet Consumption (g) & - & $0.63(0.06)$ & - & $0.53(0.09)$ \\
\hline Blood Glucose (mg/dl) & $92(10)$ & $169(8)^{\#}$ & $77(4)$ & $187(9)^{\#}$ \\
\hline Plasma Insulin (ng/mL) & $0.385(0.055)^{\mathrm{a}}$ & $2.256(0.421)^{b}$ & $0.238(0.013)^{\mathrm{a}}$ & $0.967(0.151)^{\mathrm{a}}$ \\
\hline Liver Mass (g) & $1.2(0.1)$ & $1.2(0.0)$ & $1.3(0.0)^{\$}$ & $1.3(0.0)^{\mathrm{S}}$ \\
\hline Stomach Mass (mg) & $375(41)^{\mathrm{a}}$ & $855(112)^{b}$ & $390(32)^{\mathrm{a}}$ & $573(41)^{\mathrm{a}}$ \\
\hline Empty Stomach (mg) & $206(12)$ & $158(8)$ & $180(8)$ & $135(6)$ \\
\hline Stomach Content (mg) & $169(33)^{\mathrm{a}}$ & $697(114)^{\mathrm{b}}$ & $209(26)^{\mathrm{a}}$ & $434(43)^{\mathrm{c}}$ \\
\hline
\end{tabular}

Notes: Data are presented as mean \pm SEM. Abbreviations: mg: milligrams, PBS: phosphate buffered saline, LLC: Lewis lung carcinoma, g: grams, mg/dl: milligrams per deciliter, ng/mL: nanograms per milliliter. Unpaired T-test was used to compare PBS and LLC fed pellet consumption. Two-way ANOVAs were used to compare PBS and LLC in the Fast and Fed condition. \# Main Effect of Fed. \$ Main effect of LLC. If an interaction was present; different letters were used to differentiate differences between groups. Statistically significant $\mathrm{p}<0.05$. 
incorporation in LLC fast mice was not associated with gastrocnemius muscle mass ( $\mathrm{r}=-$ $0.166, \mathrm{p}=0.695)$, eWAT mass $(\mathrm{r}=0.079, \mathrm{p}=0.853)$, nor body weight change from day 10 $(\mathrm{r}=0.259, \mathrm{p}=0.536)$. Taken together, these results suggest that fasting Akt $/ \mathrm{mTORC} 1$ signaling and protein synthesis are not associated with common indices of cachexia.

We then examined if the feeding induction of $\mathrm{Akt} / \mathrm{mTORC} 1$ and protein synthesis response was associated with cachexia progression. While AKT in LLC fed mice was not associated with gastrocnemius muscle mass $(\mathrm{r}=-0.174, \mathrm{p}=0.564)$ or body weight change from day $10(\mathrm{r}=-0.219, \mathrm{p}=0.472)$, it was negatively associated with eWAT mass $(\mathrm{r}=-$ 0.563, $\mathrm{p}=0.045$ ). Additionally, $\mathrm{rpS6}$ in LLC fed mice was not associated with gastrocnemius muscle mass $(\mathrm{r}=-0.321, \mathrm{p}=0.284)$ or body weight change from day $10(\mathrm{r}=-$ $0.232, \mathrm{p}=0.445)$, however $\mathrm{rpS6}$ was negatively associated with eWAT mass $(\mathrm{r}=-0.688$, $\mathrm{p}=0.009$ ). In summary, these data suggest that the greater increase in AKT and rpS6 was associated with reduced fat mass. While puromycin incorporation in LLC fed mice was not associated with gastrocnemius muscle mass $(\mathrm{r}=0.508, \mathrm{p}=0.076)$ or body weight change from day $10(\mathrm{r}=0.323, \mathrm{p}=0.281)$, it was positively associated with eWAT mass $(\mathrm{r}=0.709, \mathrm{p}=0.007)$ suggesting that greater increase in protein synthesis were associated with greater fat mass. Several studies have implicated fat wasting as an initial phenotype of cancer cachexia, these data would suggest that altered protein synthesis by feeding occurs early during cachexia progression. Interestingly, there is a disconnect between the association of Akt/mTORC1 and protein synthesis signaling cascades to fat wasting.

\section{Effect of feeding on AMPK signaling}

AMPK is a known metabolic checkpoint which can inhibit mTORC1 signaling. Therefore, we examined if muscle AMPK and direct downstream signaling was dysregulated by LLC and if feeding altered this response. There was a main effect for LLC to increase AMPK 1.7-fold ( $\mathrm{p}<0.001)$ compared to PBS mice, and a main effect for feeding to lower AMPK 0.6-fold ( $\mathrm{p}<0.001$ ) (Figure 3-3). FOXO3a $\mathrm{S} 413$ is a downstream target of AMPK. Feeding was sufficient to lower FOXO3a S413 in the LLC mice (Figure 3-3). Atrogin-1, a ubiquitin ligase, is commonly elevated in cachectic conditions, and it was increased with LLC and reduced by feeding (Figure 3-3). Taken together, these data highlight LLC mice have elevated muscle AMPK and feeding can lower AMPK in LLC mice. We next examined if these alterations by LLC and feeding were associated with cachexia progression. AMPK in the fed condition was not associated with body weight change from day $10(\mathrm{r}=-0.632, \mathrm{p}=0.068)$, gastrocnemius muscle mass $(\mathrm{r}=-$ $0.536, \mathrm{p}=0.137)$, nor eWAT mass $(\mathrm{r}=-0.353, \mathrm{p}=0.351)$. Given that LLC mice have overall reduced protein synthesis which coincided with elevated AMPK, and feeding was sufficient to induce protein synthesis and lower AMPK, it is within reason to speculate that the suppression of protein synthesis is impacted by AMPK. Therefore, we next sought out to investigate the fasting regulation of AMPK by LLC (Figure 3-4). 
A.

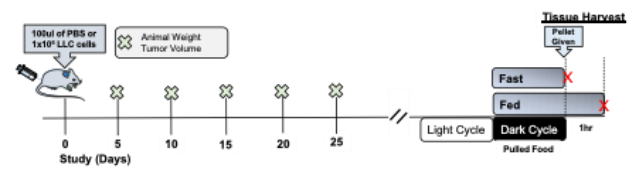

B.

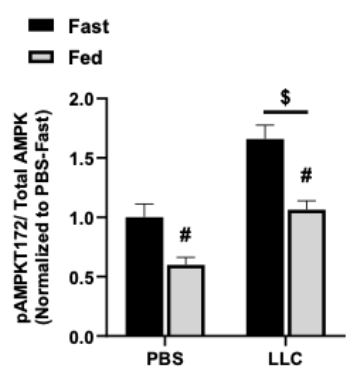

C.

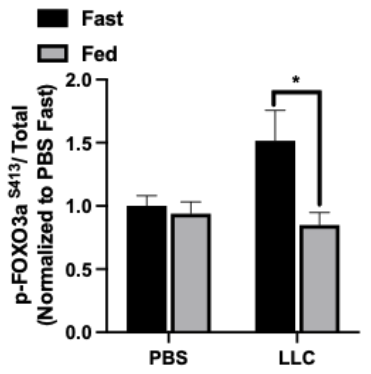

D.

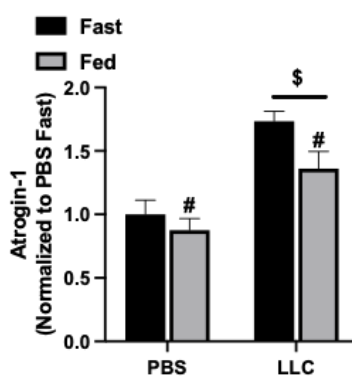

Figure 3-3. 1-hr of ad libitum feeding is sufficient to lower skeletal muscle AMPK in LLC tumor-bearing mice

Notes: Data are presented as mean \pm SEM. A. Study Design. Male C57Bl/6J mice were injected with PBS or LLC cells between 10-11 wks. of age. Tumor volume and body weight were taken every 5 days until sacrifice (28-30 days post tumor inoculation). B.Phospho-AMPK T172 in mixed gastrocnemius muscle of PBS and LLC mice in the fast and fed condition. C. Phospho-FOXO3a S413 in mixed gastrocnemius muscle of PBS and LLC mice in the fast and fed condition. D. Atrogin-1 in mixed gastrocnemius muscle of PBS and LLC mice in the fast and fed condition. Phospho- proteins were normalized to total protein. \#Main effect of Fed, \$ Main effect of LLC. If there was an interaction: *-Different between groups. 
A.

B.

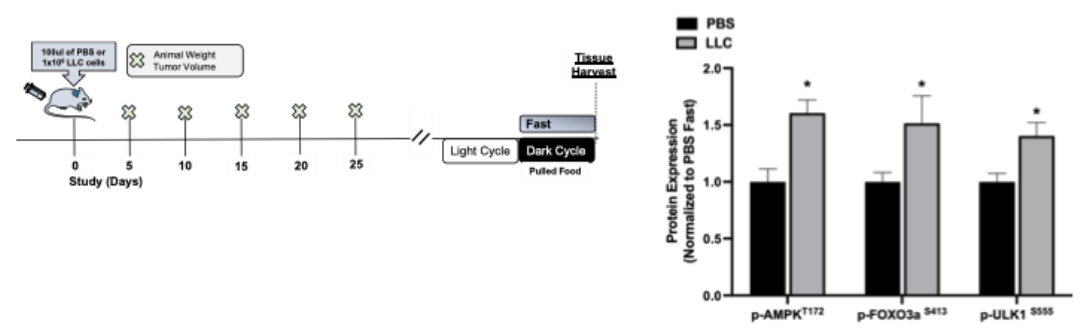

C.

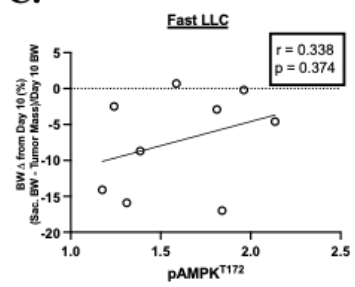

D.

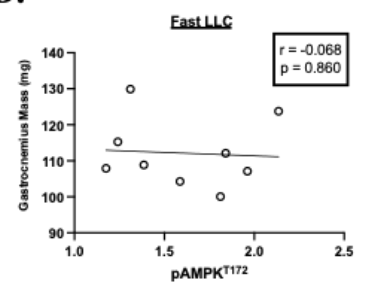

E.

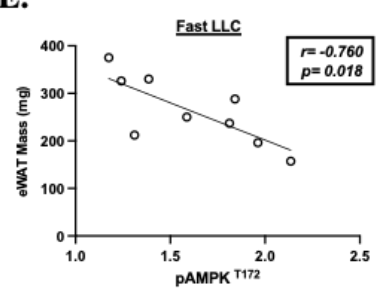

Figure 3-4. Increased AMPK signaling by fasting is associated to fat wasting in LLC tumor-bearing mice

Notes: Data are presented as mean \pm SEM. A. Study Design. Male C57B1/6J mice were injected with PBS or LLC cells between 10-11 wks. of age. Tumor volume and body weight were taken every 5 days until sacrifice (28-30 days post tumor inoculation). B.Mixed muscle AMPK signaling. C. Correlation of phospho-AMPK with body weight change from day 10. D. Correlation of phospho-AMPK with gastrocnemius mass. E.Correlation of phospho-AMPK with eWAT mass. Unpaired T-test were used to compare PBS and LLC fast mice. Phospho- proteins were normalized to total protein. * Different from PBS. Correlations were used to compare phospho-AMPK with indices of cachexia (body weight change from day 10, gastrocnemius muscle mass, and eWAT mass. 
LLC tumor-bearing mice exhibit elevated AMPK T172, ULK1 S555, and FOXO3a S413

(Figure 3-4). Fasting AMPK was not associated with body weight change from day 10 (Figure 3-4) or gastrocnemius muscle mass (Figure 3-4). Interestingly, fasting AMPK was strongly associated with eWAT mass (Figure 3-4). FOXO3a in the fast condition was not associated to body weight change from day $10(\mathrm{r}=-0.517, \mathrm{p}=0.159)$, gastrocnemius muscle mass $(\mathrm{r}=0.489, \mathrm{p}=0.182)$, nor eWAT mass $(\mathrm{r}=0.242, \mathrm{p}=0.531)$. ULK1 in the fast condition was not associated with body weight change from day 10 $(\mathrm{r}=0.511, \mathrm{p}=0.196)$, gastrocnemius muscle mass $(\mathrm{r}=0.161, \mathrm{p}=0.704)$, nor eWAT mass $(\mathrm{r}=-$ $0.252, \mathrm{p}=0.557$ ). Taken together, these data suggest that following an overnight fast, LLC tumor-bearing mice have elevated AMPK in pre-cachectic tumor-bearing mice.

\section{Early onset whole body metabolism and physical inactivity in tumor-bearing mice}

Cancer cachexia is known to disrupt whole body metabolism and physical activity. We sought to determine if there were early disruptions in physical activity and whole-body metabolism in LLC tumor-bearing mice, and if these disruptions were associated to end stage muscle signaling (Figure B-3). LLC tumor-bearing mice have increased blood glucose following a glucose tolerance test 15 days post tumor inoculation. Interestingly, there was negative association of eWAT mass at the end study with early onset glucose intolerance (Table B-1). Lastly, glucose tolerance test area under the curve was not associated with muscle AMPK associated signaling at the end study (Table B-2). LLC tumor-bearing mice exhibit early onset physical inactivity during the dark cycle (Figure B-1). Additionally, physical inactivity in LLC mice was positively associated with gastrocnemius muscle mass and fat mass at the end of study (Table B-1). As expected, lipid oxidation decreased, and carbohydrate oxidation increased during the

dark cycle in both PBS and LLC mice. There were no differences in lipid or carbohydrate oxidation between PBS and LLC mice. Interestingly, lipid nor carbohydrate oxidation was not associated with indices of cachexia (Table B-1). Taken together, LLC tumorbearing mice exhibit early onset glucose intolerance and physical inactivity, which are associated with early indices of cachexia.

\section{Muscle-specific role of AMPK}

AMPK $\alpha 1$ and AMPK $\alpha 2$ floxed mice were generously given to Dr. Carson by Dr. Hoh Jin Ko at the University of South Carolina. Mice were transferred to the University of Tennessee Health Science Center for breeding. We then crossed the AMPK $\alpha 1$ with the AMPK $\alpha 2$ to generate the AMPK $\alpha 1 \alpha 2$ floxed mouse. We then crossed the AMPK $\alpha 1 \alpha 2$ with the transgenic mouse HSA mer-cre-mer mouse. The crossing of these mice produced $\sim 50 \%$ AMPK $\alpha 1 \alpha 2$ and $50 \%$ AMPK $\alpha 1 \alpha 2$ hsac/+ (Figure 3-5). Genotyping was confirmed by tail snips after 21 days neonatal (Figure 3-5). Around 10 weeks of age, mice were given tamoxifen injection once a day for 5 consecutive days to bring the 
A.

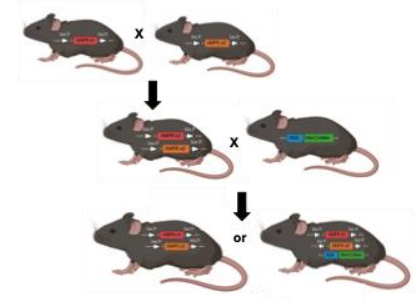

B.

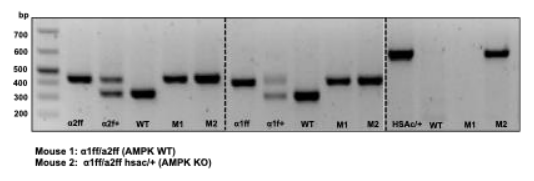

C.

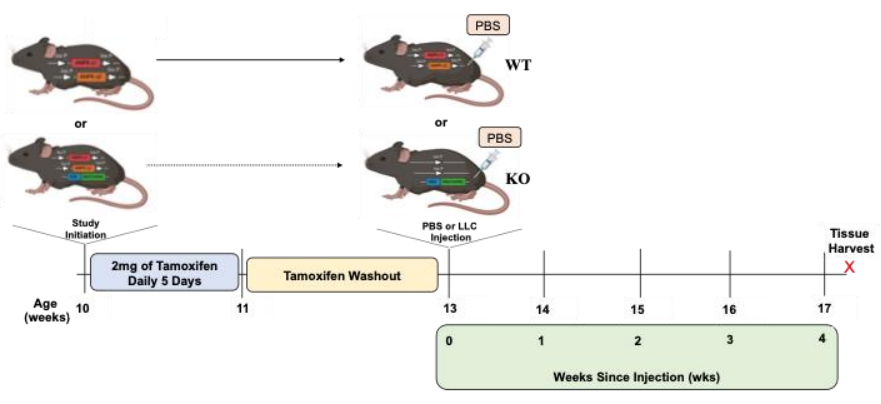

D.

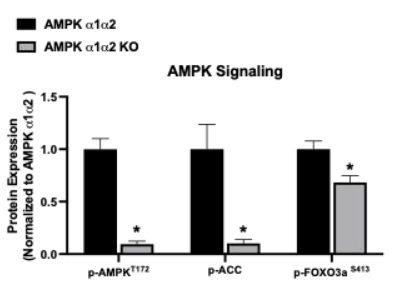

Figure 3-5. Muscle-specific AMPK loss in male PBS mice

Notes: Male C57B1/J mice were used from UTHSC breeding colony. A. Breeding schematic to produce AMPK a1+/+ a $2+/+$ with and without HSA mer-cre-mer. B. Example of tail snip confirmation of animal genotype. C. Study Design. Created with BioRender.com. D. Protein expression of phospho-AMPK loss and immediate downstream signaling in WT and KO PBS male mice. Direct downstream targets ACC and FOXO3a. N=7-8 per group. Phospho- proteins were normalized to total protein. Abbreviations: AMPK: Adenosine Monophosphate protein kinase, WT: Wildtype AMPK a1+/+ a2+/+, KO: Knockout, AMPK a1-/- a2-/-(KO), PBS: phosphate buffered saline, LLC: Lewis lung carcinoma, ULK: Unc-51 like autophagy activating kinase 1, FOXO: Forkhead box O3, p: Phosphorylation. 
LoxP-MerCreMer bound binding sites together to remove the floxed genes of interest. After a two-week washout period, mice were injected with either PBS or LLC cells and monitored for 4 weeks (Figure 3-5).

To validate the efficacy of knocking out AMPK and associated signaling. Tail snips were conducted at neonatal 21 days and mice was determined as having the double floxing of AMPK $\alpha 1 \alpha 2$ with or without the presence of hsac/+. To validate the loss of AMPK on AMPK targets we analyzed phospho-AMPK, phospho-ULKS555, FOXO3aS413, and ACC. Phospho-AMPK was decreased 91\%, phospho-ACC1 S212 decreased by $27 \%$, and FOXO3aS4 13 decreased by $32 \%$ in AMPK $\alpha 1 \alpha 2 \mathrm{KO}$ mice compared to AMPK $\alpha 1 \alpha 2$ PBS mice (Figure 3-5 and Figure B-4). Importantly, there were no differences in body weight over time, skeletal muscle mass (gastrocnemius or hindlimb muscle mass), eWAT mass, or tibia length in AMPK WT mice compared to AMPK KO mice (Table 3-4). These data highlight the ability to decrease AMPK and associated signaling in skeletal muscle, which does not alter tissue mass and body size in PBS treated male mice.

\section{Effect of AMPK loss on skeletal muscle signaling in non-tumor-bearing mice}

To understand how AMPK loss alters skeletal muscle signaling in tumor-bearing mice, we first need to determine what AMPK loss does to skeletal muscle signaling in non-tumor-bearing male mice in the fast condition. All mice were sacrificed at the start of the light cycle following an overnight 12-hr fast. First, we examined the effect of AMPK loss on autophagy associated signaling (P62, LC3BII/I, and Beclin-1). Interestingly, P62 and Beclin-1 were not altered by AMPK loss, while LC3BII/I ratio was induced. The induction of LC3BII/I ratio is due to an increase in LC3BII $(\mathrm{p}=0.006)$ without any changes in LC3BI $(p=0.514)$. AMPK has known regulation to inhibit mTORC1 therefore we examined if AMPK played a role in basal skeletal muscle anabolic signaling (Figure B-4). As expected, AKT T308 was not altered by AMPK loss. RpS6 S240/244 was increased 2.3-fold in AMPK KO mice compared to wild-type. Puromycin incorporation was increased 0.7-fold in AMPK KO mice compared to wild-type mice. Taken together, we highlight that following a 12-hr fast, AMPK is regulating rpS6 and protein synthesis. Lastly, it is well known that AMPK induces mitochondrial biogenesis. Therefore, we examined markers of mitochondrial quality control (Figure B-4). PGC1 $\alpha$ a marker of mitochondrial biogenesis was reduced by AMPK KO. Additionally, DRP1 S616 and FIS1, markers of mitochondrial fission, were reduced by AMPK KO. Interestingly, OPA-1, a marker of mitochondrial fusion, was reduced by AMPK KO. Collectively, we further support the regulation of skeletal muscle mitochondrial quality control by AMPK. 
Table 3-4. Muscle-specific AMPK loss does not alter animal characteristics in PBS treated male mice

\begin{tabular}{|c|c|c|c|}
\hline & $\mathrm{AMPK}^{\alpha 1+/+\alpha 2+/+}(\mathbf{W T})$ & $\mathrm{AMPK}^{\alpha 1-/ / \alpha 2-/-}(\mathrm{KO})$ & \\
\hline Characteristics & $\mathrm{N}=\mathbf{1 5}$ & $\mathbf{N}=\mathbf{1 7}$ & p-Value \\
\hline Age Inoculated with PBS (wks) & $14.1(0.3)$ & $13.5(0.4)$ & 0.258 \\
\hline Days in Study (days) & $26.9(0.5)$ & $25.8(0.06)$ & 0.060 \\
\hline BW Pre-tamoxifen (g) & $23.9(0.4)$ & $23.4(0.3)$ & 0.321 \\
\hline BW Post-tamoxifen (g) & $24.0(0.4)$ & $23.3(0.4)$ & 0.216 \\
\hline BW Pre-inoculation (g) & $25.9(0.5)^{*}$ & $24.4(0.6)^{*}$ & 0.058 \\
\hline BW d10 (g) & $27.0(0.4)^{*}$ & $25.6(0.6)^{*}$ & 0.091 \\
\hline BW End of Study (g) & $28.4(0.4)^{*}$ & $27.4(0.5)^{*}$ & 0.106 \\
\hline BW $\Delta$ During Tamoxifen $(\%)$ & $0.52(1.1)$ & $-0.17(0.60)$ & 0.575 \\
\hline $\mathrm{BW} \Delta$ from $\mathrm{d} 10(\%)$ & $5.6(1.3)$ & $7.2(1.5)$ & 0.424 \\
\hline Gastrocnemius Weight (mg) & $122(2)$ & $117(3)$ & 0.059 \\
\hline Tibialis Anterior Weight (mg) & $45(1)$ & $43(1)$ & 0.254 \\
\hline HMM (mg) & $203(4)$ & $195(3)$ & 0.109 \\
\hline Testes Weight (mg) & $194(3)$ & $186(5)$ & 0.234 \\
\hline Seminal Vesicle Weight (mg) & $254(8)$ & $256(12)$ & 0.892 \\
\hline Seminal Vesicle Weight (mg) & $254(8)$ & $442(49)$ & 0.752 \\
\hline eWAT (mg) & $421(45)$ & $69(2)$ & 0.876 \\
\hline Spleen Mass (mg) & $68(4)$ & $4.4(1.1)$ & 0.092 \\
\hline Plasma IL-6 (pg/ml) & $4.8(0.9)$ & $17.2(0.1)$ & 0.412 \\
\hline Tibia Length (mm) & $17.0(0.1)$ & $13.5(0.4)$ & 0.258 \\
\hline
\end{tabular}

Notes: Data are presented as Mean +/- SEM. Male C57B1/J mice were used from UTHSC breeding colony. 32 mice were randomly assigned to PBS injections with the genotypes of AMPK a1+/+ a2+/+(WT; N=15) AMPK a1-/- a2-/-(KO; N=17). Abbreviations: SEM: Standard error of the mean, g: grams, mg: milligrams, BW: body Weight, TW: tumor weight, $\Delta$ : Change, \%: Percent, PBS: Phosphate Buffered Saline, mm: millimeters, HMM: hindlimb muscle mass. Statistical Significance $p<0.05$. Unpaired T-Test was used to compare PBS WT and PBS KO male mice. Two-way repeated measures ANOVA $(2 \mathrm{x}$ 5 ) was used to compare body pre-tamoxifen, post-tamoxifen, pre-inoculation, BW Day 10 , and BW end of study. There was a main effect of time without differences between WT and KO mice. When determining which groups were different, *Notes statistically significant from all other time points within WT and KO mice. 


\section{Validation of muscle-specific AMPK loss in LLC tumor-bearing mice}

To validate the efficacy of knocking out AMPK and associated signaling in LLC tumor-bearing mice. Tail snips were conducted at neonatal 21 days and mice was determined as having the double floxing of AMPK $\alpha 1 \alpha 2$ with or without the presence of hsac/+. After tamoxifen washout, mice were injected with either PBS or LLC cells and sacrificed 4-weeks later (Figure 3-6). We first validated all mice by showing the absence of total AMPK in PBS and LLC AMPK WT and AMPK KO male mice (Figure 3-6). Importantly, AMPK loss in LLC tumor-bearing mice was sufficient to reduce the increased in phospho-AMPK, FOXO3a, and Atrogin-1 (Figure 3-6).

\section{Muscle-specific AMPK loss in LLC tumor-bearing male mice does not alter tumor growth, tumor mass, or indices of cachexia}

Tumor volume increased from days $10-25$ in AMPK WT and KO mice $(\mathrm{p}<0.001)$ with no difference between WT and KO mice $(\mathrm{p}=0.402)$ (Figure 3-7). There were no differences in tumor growth rate between WT and KO LLC mice $(p=0.211)$. There were no differences in tumor mass between WT and KO LLC mice $(\mathrm{p}=0.109)$. There was a main effect of time $(\mathrm{p}<0.001)$ for body weight during the study to increase at each time point (Table 3-5). When accounting for tumor mass, there was a main effect for LLC mice to have decreased body weight at the end of the study $(\mathrm{p}<0.001)$, without differences between WT and KO mice $(p=0.131)$. There was a main effect for LLC mice to have more body weight change from day $10(p<0.001)$, without differences between WT and KO mice ( $p=0.713)$. There was a main effect for LLC mice to have decreased gastrocnemius muscle $(\mathrm{p}<0.001)$, without differences between WT and KO mice $(p=0.076)$. There was a main effect for LLC mice to have decreased eWAT mass $(p=0.002)$, without differences between WT and KO mice $(p=0.458)$.

\section{Effect of AMPK loss on Akt/mTORC1 signaling and protein synthesis in LLC tumor-bearing male mice}

Given the known suppression of basal Akt/mTORC1 signaling and protein synthesis in LLC mice compared to PBS mice, and AMPK's known regulation of mTORC1 signaling it is within reason to speculate that in tumor-bearing mice when AMPK is elevated, by lowering the elevated AMPK signaling we can improve mTORC1 signaling and protein synthesis. Therefore, we examined the effect of AMPK loss in the fasting condition in PBS and LLC mice (Figure 3-8). There was a main effect for LLC mice to have decreased puromycin incorporation compared to PBS $(\mathrm{p}=0.008)$ and a main effect for $\mathrm{KO}$ to increase puromycin incorporation $(\mathrm{p}=0.027)$. There was a main effect for LLC mice to have decreased rps6 compared to PBS $(p=0.008)$ and a main effect for $\mathrm{KO}$ to increase rps6 ( $\mathrm{p}=0.027$; Figure 3-8). There was a main effect for LLC mice to have decreased AKT compared to PBS ( $\mathrm{p}=0.006)$, without any effects of $\mathrm{KO}(\mathrm{p}=0.353$; Figure 3.8). Taken together we highlight that AMPK is regulating 
A.

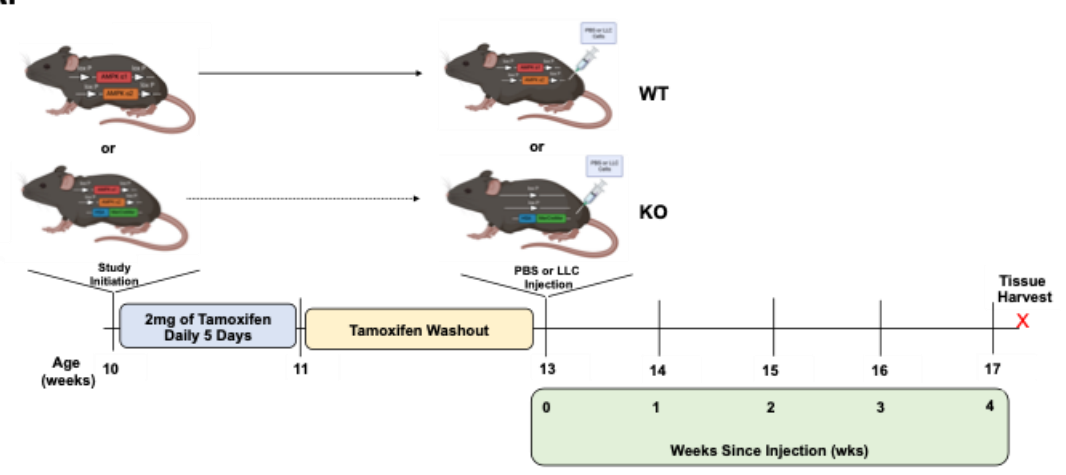

B.

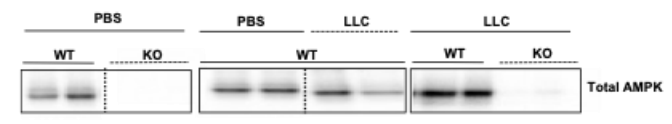

C.

PBS - AMPK WT
$\square$ PBS - AMPK KO

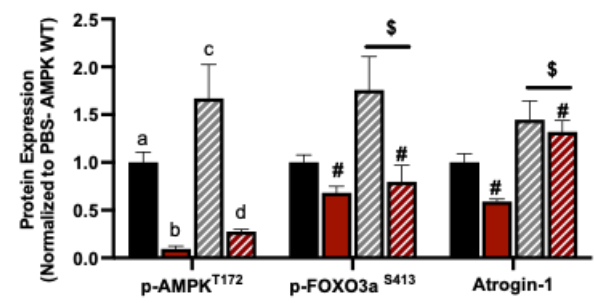

Figure 3-6. Increased AMPK signaling can be reduced by muscle-specific AMPK loss in LLC tumor-bearing mice

Notes: Data are presented as mean \pm SEM. A. Study Design. Created with BioRender.com. Male C57B1/6J mice were injected with PBS or LLC cells between 1011 wks. of age. Tumor volume and body weight were taken every 5 days until sacrifice (28-30 days post tumor inoculation). B. Validation of total AMPK loss in KO PBS and LLC mice. C.-Protein expression of phospho-AMPK loss and immediate downstream signaling in WT and KO PBS and LLC male mice. $\mathrm{N}=7-11$ per group. Phospho- proteins were normalized to total protein. Abbreviations: BW: body weight, mg: milligrams, PBS: phosphate buffered saline, LLC: Lewis lung carcinoma, g: grams. Unpaired t-test was used to compare PBS to LLC. Two-way ANOVA (PBS or LLC vs WT or KO) was used. If there was an interaction; different letter note groups are different. \# Main effect of KO, \$ Main effect of LLC. 
A.

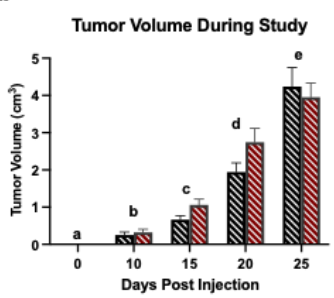

D.

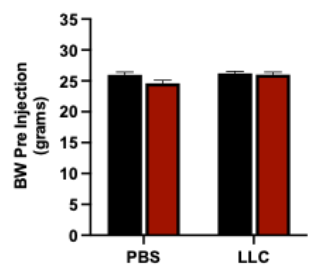

F.

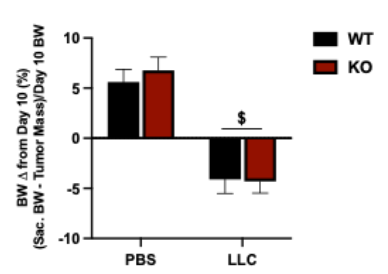

B.

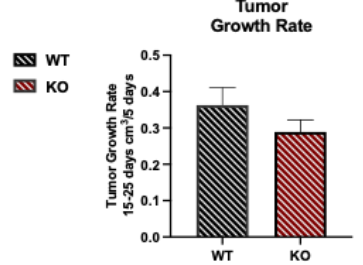

c.

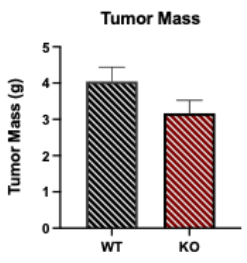

E.
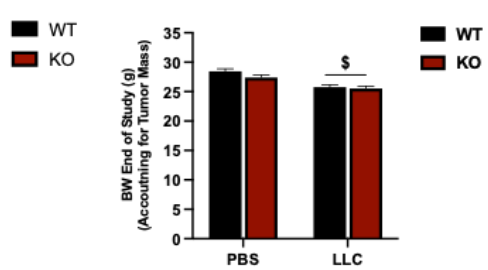

G.

H.

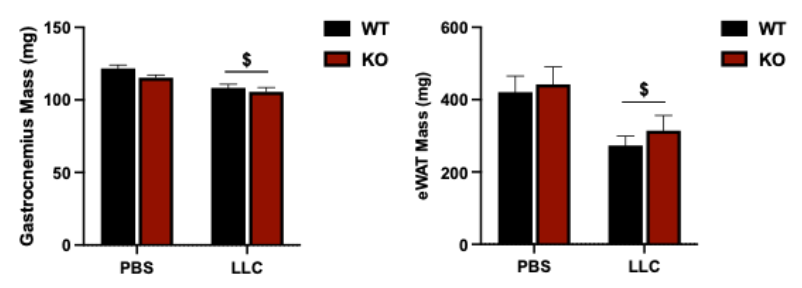

Figure 3-7. Muscle-specific AMPK loss in LLC tumor-bearing mice does not alter tumor mass, tumor growth, or indices of cachexia

Notes: Data are presented as mean \pm SEM. A. Study Design. Male C57B1/6J mice were injected with PBS or LLC cells between 10-11 wks. of age. Tumor volume and body weight were taken every 5 days until sacrifice (28-30 days post tumor inoculation). B.Tumor growth rate from days 15-25. C. Tumor Mass at sacrifice. D. Body weight pre injection in PBS and LLC WT and KO mice. E. Body weight end of study accounting for tumor mass in PBS and LLC WT and KO mice. F. Body weight change from Day 10 post tumor inoculation. Body weight is calculated as the sacrifice body weight minus the tumor mass divided by day 10 body weight. G. Gastrocnemius muscle mass at sacrifice. H. eWAT mass at sacrifice. Abbreviations: BW: body weight, mg: milligrams, PBS: phosphate buffered saline, LLC: Lewis lung carcinoma, g: grams. Unpaired t-test was used to compare PBS to LLC. Two-way (Genotype x LLC) repeated measures ANOVA. $\$$ Main Effect of LLC. Two-way repeated measures ANOVA was used to compare tumor volume overtime in LLC WT and KO mice. Different letters note a difference between time points within each condition. 
Table 3-5. Effect of muscle-specific AMPK loss on animal characteristics in LLC tumor-bearing mice

\begin{tabular}{|c|c|c|c|c|}
\hline \multirow[b]{2}{*}{ Characteristics } & \multicolumn{2}{|c|}{ PBS } & \multicolumn{2}{|c|}{ LLC } \\
\hline & $\begin{array}{c}\text { WT } \\
\mathrm{N}=15\end{array}$ & $\begin{array}{c}\text { KO } \\
\mathbf{N}=17\end{array}$ & $\begin{array}{c}\text { WT } \\
\mathrm{N}=16\end{array}$ & $\begin{array}{c}\text { KO } \\
\mathbf{N}=19\end{array}$ \\
\hline Age Inoculated (wks) & $14.1(0.3)$ & $13.5(0.4)$ & $14.0(0.3)$ & $13.9(0.3)$ \\
\hline BW Pre-tamoxifen (g) & $23.9(0.4)$ & $23.4(0.3)$ & $23.090 .5)$ & $23.5(0.4)$ \\
\hline BW Post-tamoxifen (g) & $24.0(0.4)$ & $23.3(0.4)$ & $23.9(0.5)$ & $24.5(0.4)$ \\
\hline Days in Study (days) & $26.7(0.3)$ & $25.9(0.3)$ & $25.8(0.4)^{@}$ & $25.4(0.3)^{@}$ \\
\hline BW Pre-inoculation (g) & $26.0(0.4)^{\mathrm{a}}$ & $24.6(0.5)^{\mathrm{a}}$ & $26.2(0.3)^{\mathrm{a}, \mathrm{b}}$ & $26.0(0.4)^{\mathrm{a}}$ \\
\hline BW d10 (g) & $26.7(0.4)^{b}$ & $25.6(0.6)^{b}$ & $26.8(0.4)^{\mathrm{a}}$ & $26.7(0.5)^{b}$ \\
\hline BW End of Study (g) & $28.4(0.4)$ & $27.4(0.5)^{\wedge}$ & $29.6(0.4)^{@}$ & $28.7(0.5)^{\varrho^{\wedge}}$ \\
\hline Tumor Weight (TW) (g) & - & - & $3.4(0.4)$ & $3.2(0.4)$ \\
\hline BW - TW (g) & $28.4(0.4)^{\mathrm{c}}$ & $27.4(0.5)^{\mathrm{c}}$ & $25.8(0.4)^{@ b}$ & $25.5(0.4)^{@ a \mathrm{a}}$ \\
\hline $\mathrm{BW} \Delta$ from d10 $(\%)$ & $5.6(1.3)$ & $7.2(1.5)$ & $-4.1(1.3)^{@}$ & $-4.3(1.2)^{@}$ \\
\hline Tumor Volume d15 $\left(\mathrm{cm}^{3}\right)$ & - & - & $0.708(0.104)^{\mathrm{a}}$ & $1.062(0.153)^{\mathrm{a}}$ \\
\hline Tumor Volume d20 $\left(\mathrm{cm}^{3}\right)$ & - & - & $1.942(0.249)^{\mathrm{b}}$ & $2.742(0.370)^{b}$ \\
\hline Tumor Volume d25 $\left(\mathrm{cm}^{3}\right)$ & - & - & $4.239(0.508)^{\mathrm{c}}$ & $3.951(0.378)^{\mathrm{c}}$ \\
\hline Tumor Growth Rate $\left(\mathrm{cm}^{3} / 5\right.$ days) & - & - & $0.363(0.049)$ & $0.289(0.033)$ \\
\hline Soleus Weight (mg) & $7.2(0.3)$ & $7.9(0.2)$ & $7.7(0.4)$ & $7.5(0.3)$ \\
\hline Gastrocnemius Weight (mg) & $122(2)$ & $115(3)$ & $107(3)^{@}$ & $106(3)^{@}$ \\
\hline Tibialis Anterior Weight (mg) & $45(1)$ & $43(1)$ & $38(1)^{@}$ & $38(1)^{@}$ \\
\hline HMM (mg) & $203(4)$ & $195(3)$ & $177(4)^{@}$ & $176(4)^{@}$ \\
\hline Testes Weight (mg) & $194(3)$ & $186(5)$ & $183(3)$ & $183(4)$ \\
\hline Seminal Vesicle Weight (mg) & $254(8)$ & $256(12)$ & $210(11)^{@}$ & $218(10)^{@}$ \\
\hline eWAT (mg) & $421(45)$ & $442(49)$ & $262(27)^{@}$ & $314(42)^{@}$ \\
\hline Spleen Mass (mg) & $68(4)$ & $69(2)$ & $229(28)^{\#}$ & $162(14)^{\#}$ \\
\hline Plasma IL-6 (pg/ml) & $4.8(0.9)$ & $7.3(1.1)$ & $18.0(3.0)^{@}$ & $16.3(2.1)^{@}$ \\
\hline Tibia Length (mm) & $17.0(0.1)$ & $17.2(0.1)$ & $16.9(0.2)$ & $17.2(0.1)$ \\
\hline
\end{tabular}

Notes: Data are presented as mean \pm SEM. Male C57B1/J mice were used from UTHSC breeding colony. 67 mice were randomly assigned to either PBS (N:32) or LLC (N:35) then at the time of sacrifice were either fasted or Fed. Abbreviations: SEM: Standard error of the mean, $\mathrm{cm}^{3}$ : centimeters cubed, mg: milligrams, BW: body Weight, TW: tumor weight, $\Delta$ : Change, \%: Percent, LLC: Lewis lung carcinoma, PBS: Phosphate Buffered Saline, mm: millimeters, HMM: Hindlimb muscle mass. Statistical Significance $\mathrm{p}<0.05$. Two-way ANOVA ( 2 Treatments $\mathrm{x} 2$ nutrient conditions) was used to compare across groups. ME of Genotype: ^, ME of LLC@, One-way repeated measures ANOVA was used to compare body weight overtime within each treatment and genotyped. Different letters mean there was an interaction and different from other letters. One-way ANOVA was used to compare tumor volume overtime within each genotype group of LLC mice. Different letters mean there was an interaction and different from other letters. 


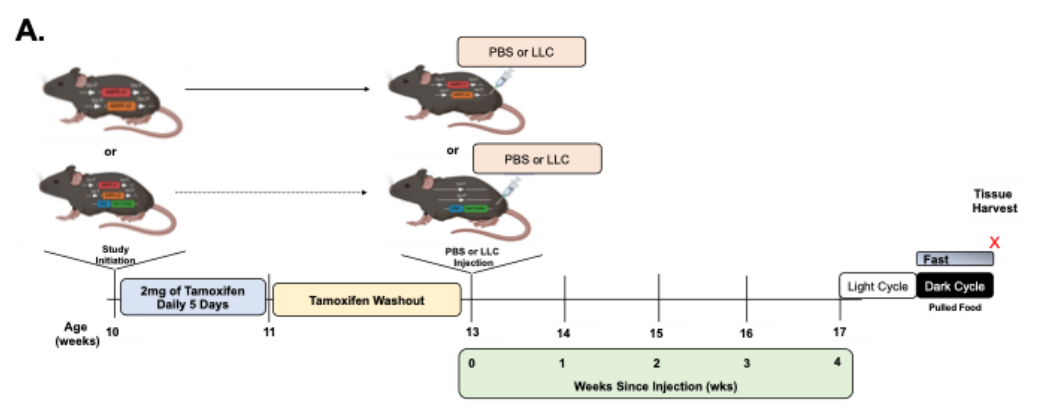

B.

B. $\square \mathrm{wT}$

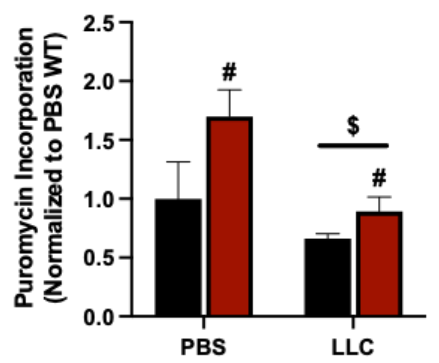

D.

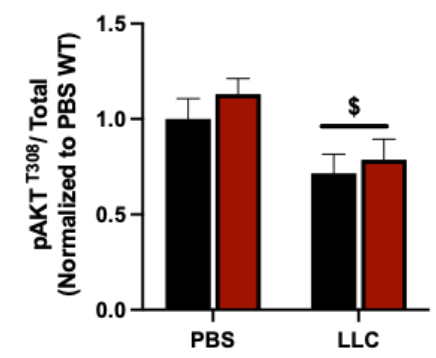

c.

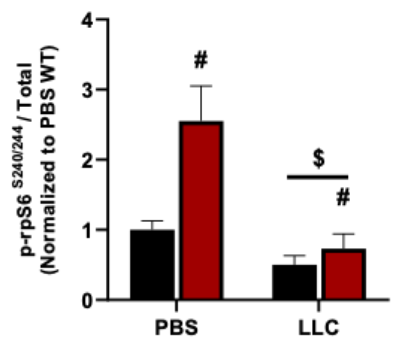

Figure 3-8. Muscle-specific AMPK loss is sufficient to increase muscle protein synthesis in LLC tumor-bearing mice following a $12 \mathrm{hr}$ fast

Notes: Data are presented as mean \pm SEM. A. Study Design. Male C57Bl/6J mice were injected with PBS or LLC cells between 10-11 wks. of age. Tumor volume and body weight were taken every 5 days until sacrifice (28-30 days post tumor inoculation). Mice were fasted during the dark cycle and sacrificed at the start of the light cycle following a $12 \mathrm{hr}$ fast. B. Puromycin incorporation in PBS and LLC AMPK WT and AMPK KO mice. C. Phospho-rpS6 to total ratio in PBS and LLC AMPK WT and AMPK KO mice. D Phospho-AKT to total ratio in PBS and LLC AMPK WT and AMPK KO mice. White gastrocnemius muscle was for protein expression. Phospho- proteins were normalized to total protein. Abbreviations: PBS: phosphate buffered saline, LLC: Lewis lung carcinoma. Two-way (Genotype x LLC) was used. \$ Main Effect of LLC. \# Main Effect of AMPK KO. 
the fasting suppression of mTORC1 signaling and protein synthesis in LLC tumor bearing mice.

\section{Effect of feeding on skeletal muscle protein turnover}

Due to different animal cohorts being used because of tamoxifen use, we first need to validate the feeding response in PBS mice (Table 3-6). Feeding suppressed AMPK 0.4-fold without altering common downstream targets of AMPK (Figure B-5). Regarding autophagy associated signaling, LC3BII/I ratio was reduced with feeding which was due to an increase in LC3BI ( $p=0.002)$ without changes in LC3B II ( $p=0.518)$. As expected, feeding increased AKT 0.4-fold, rpS6 6.2-fold, and protein synthesis 0.9fold compared to fast mice (Figure B-3). Lastly, feeding did not alter PGC1 $\alpha$ or OPA-1, but was sufficient to marginally reduce DRP1 S616 0.3-fold and FIS-1 0.4-fold (Figure B-3). Taken together, these data highlight the anabolic response of 1-hr feeding in nontumor-bearing mice that received tamoxifen confirming that tamoxifen treatment (used to knockout AMPK in skeletal muscle) did not alter feeding associated signaling.

\section{Effect of muscle-specific AMPK loss on skeletal muscle's feeding regulation of skeletal muscle protein turnover}

We have previously shown that feeding and muscle-specific AMPK loss increased muscle protein synthesis independently. We next sought to determine if AMPK loss regulated the feeding response. AMPK loss suppresses AMPK, ACC, and FOXO3a, which is not further lowered by feeding in the tumor-bearing mouse (Figure B-6). To validate the feeding response, AKT phosphorylation increased in both fed conditions (Figure 3-9). Interestingly, AKT phosphorylation was further increased in the fed condition in AMPK KO mice (Figure 3-9). RpS6 was increased by feeding in AMPK WT mice compared to AMPK WT fast mice (Figure 3-9). AMPK KO fast and fed mice had increased rpS6 compared to AMPK WT fast mice. Interestingly, puromycin incorporation was not further increased with feeding in AMPK KO PBS mice (Figure 3-9). Taken together, these data suggest that AMPK loss does not further induce the mTORC1 and protein synthesis response to feeding in PBS mice. Interestingly, LC3BII/I ratio was suppressed by feeding, and occurs independent of AMPK (Figure B-5). We next sought to determine AMPK's regulation of the feeding response in LLC mice. We have previously shown that Akt/mTORC1 signaling and protein synthesis are suppressed in fast and fed LLC mice compared to PBS mice. Additionally, we have shown that the loss of AMPK in LLC mice can improve mTORC1 and protein synthesis in the fast condition compared to PBS. We report that there were main effects of feeding to increase AKT and rpS6 phosphorylation and protein synthesis without any effects by AMPK KO (Figure 3-9). These data identify that AMPK's regulation of feeding's regulation on muscle Akt/mTORC1 and protein synthesis is not additive, suggesting that feeding and AMPK loss in the fast condition are independently capable of increasing muscle proteins synthesis. 
Table 3-6. Whole-body response to feeding in PBS and LLC male mice

\begin{tabular}{lcccccc}
\hline & \multicolumn{2}{c}{ PBS } & & \multicolumn{2}{c}{ LLC } \\
\cline { 2 - 3 } \cline { 5 - 6 } Outcomes & Fast & Fed & & Fast & Fed \\
\hline Pellet Consumption $(\mathrm{g})$ & $\mathbf{N}=\mathbf{1 7}$ & - & $\mathbf{N}=\mathbf{1 5}$ & & $\mathbf{N = 2 0}$ & $\mathbf{N = 1 5}$ \\
Blood Glucose $(\mathrm{mg} / \mathrm{dl})$ & $99(5)$ & $222(9) \#$ & & $90(5)$ & $222(8) \#$ \\
Plasma Insulin $(\mathrm{ng} / \mathrm{mL})$ & $0.211(0.006)^{\mathrm{a}}$ & $0.830(0.083)^{\mathrm{b}}$ & & $0.253(0.025)^{\mathrm{a}}$ & $0.439(0.076)^{\mathrm{a}}$ \\
Stomach Mass $(\mathrm{mg})$ & $428(18)$ & $677(68) \#$ & & $349(22)$ & $637(43) \#$ \\
Empty Stomach $(\mathrm{mg})$ & $154(4)$ & $147(2) \#$ & & $156(8)$ & $136(5)$ \\
Stomach Content $(\mathrm{mg})$ & $274(19)$ & $530(67) \#$ & & $192(20)$ & $501(40) \#$ \\
\hline
\end{tabular}

Notes: There were no effects of AMPK KO on whole-body response to feeding therefore PBS and LLC AMPK WT and AMPK KO mice in the fast or fed condition are combined to show the summary data. Data are presented as Mean (SEM). Abbreviations: mg: milligrams, PBS: phosphate buffered saline, LLC: Lewis lung carcinoma, g: grams, mg/dl: milligrams per deciliter, ng/mL: Nanograms per milliliter, and ME: Main Effect. Unpaired T-test was used to compare PBS and LLC fed pellet consumption. Two-way ANOVAs were used to compare PBS and LLC in the Fast and Fed condition. \#ME of Fed. If an interaction was present; different letters were used to differentiate differences between groups. Statistically significant $p<0.05$. 
A.

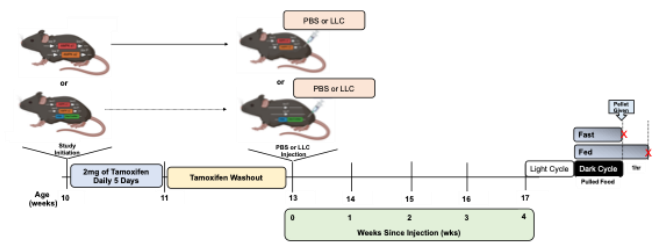

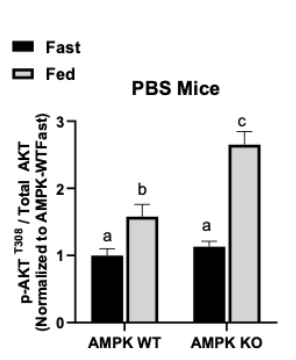

E.

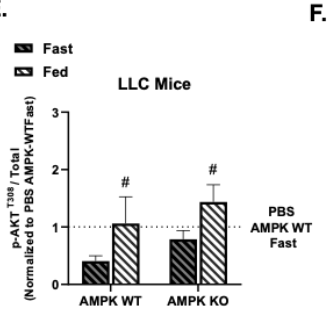

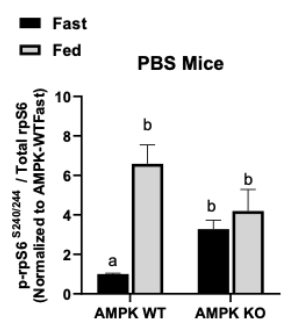

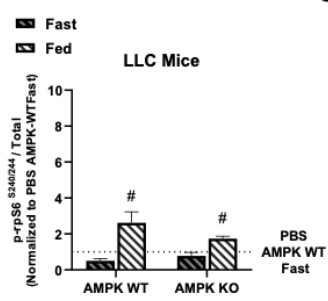

D.

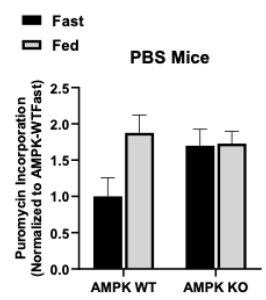

G.

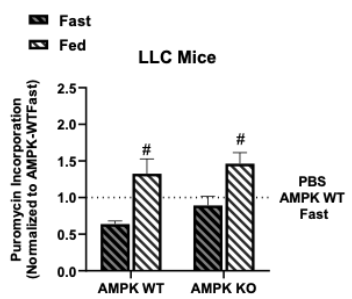

Figure 3-9. Effect of muscle-specific AMPK loss on skeletal muscle's feeding regulation of $\mathrm{Akt} / \mathrm{mTORC1}$ signaling and protein synthesis

Notes: Data are presented as mean \pm SEM. A. Study Design. Male C57B1/6J mice were injected with PBS or LLC cells between 10-11 wks. of age. Mice were fasted during the dark cycle and sacrificed at the start of the light cycle in the fast condition or given access to a food pellet for $1 \mathrm{hr}$ (fed) then sacrificed. B. Phospho-AKT to total ratio in PBS AMPK WT and AMPK KO mice in the fast or fed condition. C. Phospho-rpS6 to total ratio in PBS AMPK WT and AMPK KO mice in the fast or fed condition. D. Puromycin incorporation in PBS AMPK WT and AMPK KO mice in the fast or fed condition. E.Phospho-AKT to total ratio in LLC AMPK WT and AMPK KO mice in the fast or fed condition. F. Phospho-rpS6 to total ratio in LLC AMPK WT and AMPK KO mice in the fast or fed condition. G. Puromycin incorporation in LLC AMPK WT and AMPK KO mice in the fast or fed condition. White gastrocnemius muscle was for protein expression. Phospho- proteins were normalized to total protein. Abbreviations: PBS: phosphate buffered saline, LLC: Lewis lung carcinoma. Two--way (Genotype x LLC) was used \#Main Effect of Fed. If there was an interaction, different letters were used to determine what groups were different 


\section{Discussion}

Cancer-induced cachexia disrupts skeletal muscle protein turnover in the basal condition (decreasing protein synthesis and increasing protein degradation). In preclinical models, our laboratory has demonstrated altered protein turnover through the suppression of anabolic signaling via mTORC1 which coincided with the chronic activation of AMPK (White, Baynes, et al., 2011). We have recently reported that mTORC1 signaling exhibits diurnal fluctuations in response to changes throughout the day in feeding and activity (Counts et al., 2020). Furthermore, the cachectic environment disrupts these diurnal fluctuations, and suggest a link between disruptions in feeding and activity behaviors with the progression of cachexia and altered mTORC1 signaling (Counts et al., 2020). Taken together, cachexia suppresses muscle protein synthesis which coincides with an increase in muscle AMPK signaling in the basal condition. Therefore, we extended previous studies by examining if tumor-bearing mice had the capacity for feeding to increase muscle protein synthesis. We report that pre-cachectic tumor-bearing mice increased muscle $\mathrm{Akt} / \mathrm{mTORC} 1$ signaling and protein synthesis in response to feeding. Interestingly, feeding was sufficient to lower the elevated AMPK in pre-cachectic tumor-bearing mice. Tumor-bearing mice have overall suppressed Akt/mTORC1 signaling and protein synthesis, independent of the fast or fed condition and AMPK regulates the fasting suppression of muscle protein synthesis in pre-cachectic tumor-bearing mice. These findings provide novel insight into the regulation of Akt/mTORC1 signaling and protein synthesis in response to feeding.

Anabolic resistance is defined as the reduced stimulation to an anabolic stimulus (Morton et al., 2018). Cancer cachexia has been routinely considered to produce anabolic resistance in skeletal muscle (Hardee et al., 2017). It is generally understood that cancer cachexia induces muscle catabolic signaling and is consistent within the field, however far less is understood about anabolic signaling (Penna, Ballaro, Beltra, et al., 2019). To date, the evidence is equivocal, and this can largely be attributed to the variability within the cancer patient population (Antoun \& Raynard, 2018; Paulussen et al., 2021). For example, in the patient population considerations such as cancer type, treatments, time since diagnosis, age, and stage of cachexia are contributing to the equivocal patient data. Furthermore, in rodent models of cancer cachexia, we know that suppressed anabolic signaling is associated to cancer cachexia progression (Hardee, Counts, et al., 2018; White, Baynes, et al., 2011) and glucose administration (White et al., 2013). Importantly, recent data has highlighted the disruption in diurnal behaviors and muscle mTORC1 signaling in cachectic mice (Counts et al., 2020) therefore we extend these findings by reporting that LLC tumor-bearing mice can induce $\mathrm{Akt} / \mathrm{mTORC} 1$ signaling and protein synthesis in response to feeding. It is possible, that the increased amino acid availability from feeding induced mTORC1 and protein synthesis, and it is well known that feeding induces insulin to activate AKT; both identifying that pre-cachectic mouse muscle has the ability to response to feeding. Future studies are required to examine if insulin and amino acid availability are both required to induce muscle protein synthesis in pre-cachectic tumor-bearing mice. In agreement with previous studies, we show that mTORC1 signaling and protein synthesis were overall lower in LLC tumor-bearing mice, independent of the fast or fed condition. We also show that the suppressed mTORC1 
signaling is associated to fat mass; consistently mentioned as a phenotypic change that occurs early during cancer cachexia progression. It is interesting to speculate that cachectic skeletal muscle has the capacity to respond to an anabolic stimulus and improving the basal condition (herein the fast condition) is of more importance. Furthermore, herein we used a robust anabolic stimulus of a mixed meal and future studies should investigate if single nutrients are sufficient, and if cachexia progression disrupts the response.

Our lab and others have shown that the cachectic environment induces chronic activation of aberrant AMPK (Penna, Ballaro, Martinez-Cristobal, et al., 2019; White, Baynes, et al., 2011). Feeding is sufficient to lower muscle AMPK and the loss of AMPK is sufficient to induce mTORC1 signaling (Bujak et al., 2015). Therefore, we wanted to examine if the suppressed mTORC1 signaling in the basal condition was regulated by AMPK. We used the skeletal muscle inducible mouse to knockout AMPK only in skeletal muscle (Fix et al., 2021). We found that muscle-specific AMPK loss in the tumor-bearing mouse was sufficient to induce mTORC1 signaling and protein synthesis, suggesting that fasting suppression of $\mathrm{mTORC} 1$ and protein synthesis in tumor-bearing mice is in part, regulated by AMPK. These findings have significant implications because we know AMPK is elevated across a wide range of pre-clinical cachexia models and in the patient population, thus identifying to be a consistent cachexia phenotype in muscle. While we highlight the regulation of AMPK in skeletal muscle, given the range of functions of AMPK we are not suggesting developing pharmacological targets to inhibit AMPK but report a regulatory point of interaction. Future studies should determine the direct connection between AMPK and $\mathrm{MTORC} 1$ to provide a more targetable approach. Additionally, we are reporting the effects of AMPK loss, it would also be beneficial to determine what is activating AMPK during fasting in cachectic skeletal muscle in hopes of finding the regulator of AMPK in pre-cachectic muscle. Feeding is a sufficient stimulus to lower AMPK. We extend these findings by identifying that tumor-bearing mice can lower the elevated muscle AMPK by feeding. Additionally, in the fasting condition, mTORC1 signaling and protein synthesis are suppressed which can be improved by AMPK loss. Interestingly, we found that AMPK loss and feeding are not additive in further increasing mTORC1 signaling and protein synthesis in tumor or nontumor-bearing mice. It is interesting to speculate if mTORC1 signaling and protein synthesis had achieved their peak activity, thus not allowing for an additive effect. This would further support the idea of completing multiple anabolic insults to improve anabolic signaling. It is possible that $\mathrm{TSC} 1 / 2$ is the regulatory point of interaction because AMPK and AKT both signaling through TSC1/2 to alter mTORC1, however this is outside the scope of this study and requires further investigation. These findings are important because it suggest that one of these stimuli are sufficient to improve muscle protein synthesis, but together are not additive. Future studies should further understand why AMPK loss and feeding do not additively increase muscle protein synthesis in precachectic mice. Lastly, future studies should determine if cachectic skeletal muscle has the capacity to increase muscle protein synthesis in response to feeding.

Skeletal muscle AMPK has known regulation to not only inhibit mTORC1, but can also induce glycogen synthesis, autophagy, and mitochondrial biogenesis (Steinberg 
\& Jorgensen, 2007). While whole body AMPK loss is embryonic lethal (Viollet et al., 2009), muscle-specific loss of AMPK has a range of phenotypes and functions dependent on the isoforms lost (Kjobsted et al., 2018). In summary, the loss of AMPK subunit $\alpha$ or $\beta$ result in impaired muscle function and substrate utilization whereas $\gamma$ functions are less known because $\alpha$ or $\beta$ will compensate. Interestingly, muscle mass changes with subunit loss are inconsistent, likely due to knockout durations and efficacy of the knockout and subunit compensation. We report that AMPK loss did not prevent muscle wasting in LLC mice, despite elevated mTORC1 signaling and protein synthesis. There are several potential reasons for this; first, there were no differences in tumor mass or growth rate in LLC AMPK WT and LLC AMPK KO mice therefore the tumor and its surrounding environment are still developing thereby secreting tumor derived factors that can alter the muscle's environment. In addition, skeletal muscle mTORC1 and protein synthesis oscillate throughout the day in response to feeding and activity. It is interesting to speculate that throughout the day, mTORC1 and protein synthesis are similar in AMPK $\mathrm{KO}$ and AMPK WT mice, and once there is a stress (herein used as a $12 \mathrm{hr}$ fast), the stress is less in the AMPK KO mice. It is has been hypothesized that basal lowering of mTORC1 and protein synthesis overtime can result in decreased muscle mass (Yoon, 2017), possibly further supporting the evidence that LLC AMPK KO mice might not be regulating the daily fluctuations in $\mathrm{mTORC} 1$ and protein synthesis, but only elicit regulation during stressed conditions. Nonetheless, this does not discount our data as we highlight that when we stress the system by a $12 \mathrm{hr}$ fast in tumor-bearing mice, we can induce muscle protein synthesis by knocking out muscle AMPK. In summary, regulators of AMPK and AMPK's regulation are very complex, future studies should determine what upstream regulators and downstream effectors are eliciting altered muscle signaling, as this would provide a more targetable outcome.

Skeletal muscle AMPK is known to directly target ACC, FOXO3a, and ULK1. We report that AMPK subunit $\alpha$ loss reduces downstream ACC and FOXO3a, without altering ULK1. Phosphorylation of ACC S79 has known regulation to inhibit ACC's function causing a reduction in malonyl-CoA and increase fatty acid oxidation and phosphorylation of FOXO3a S413 activates transcription of energy-producing pathways. Interestingly, we report that ULK1S555 was not reduced by AMPK KO mice. It's interesting to speculate the effect of AMPK loss on its downstream mechanisms. For example, muscle AMPK subunit $\alpha$ loss does not alter basal whole body oxygen consumption or fuel utilization, whereas muscle AMPK subunit $\alpha$ loss does alter whole body and muscle's response to fasting and exercise (Fentz et al., 2015). Alternatively, AMPK subunit $\beta$ loss impairs volitional activity and mitochondrial biogenesis. With respect to signaling, AMPK subunit $\beta$ suppressed ULK1S555 and ACC2, while AMPK subunit $\alpha$ loss does not alter ULKS555 but does reduce ACC and FOXO3a (Fix et al., 2021). It is interestingly to speculate the role of each subunit under healthy conditions, but due to the compensatory mechanism of AMPK this might provide difficulty. Additionally, given the known elevation in AMPK and associated signaling in cachectic skeletal muscle, understanding the functionality of each AMPK subunit during differential conditions (herein we used fasting) can aid in providing a more therapeutic target. However, future studies are warranted to understand AMPK subunit function during cancer cachexia progression. 
Each study is not without limitations. A limitation of this study is that we examined males, not females. Sex as a biological variable, especially during cancer cachexia has only minimally been investigated. While this warrants further examination there are several considerations that need to be addressed. First, we used tamoxifeninducible muscle-specific knockout mice, tamoxifen binds to the estrogen receptors throughout the body thereby inhibiting the receptors effects on tissues. It has yet to be considered if the sudden loss of estrogen associated signaling impact on skeletal muscle, in an environment with high estrogen levels, females. Next, indices of cachexia are less common in female mice compared to male mice, which has been linked to hypogonadism. Additionally, there is a possibility that the 1-hr time difference between fed and fast conditions could intrinsically be different. However, there is sufficient circadian rhythm data to identify that 1-hr difference between sacrifice times will not elicit an intrinsic difference in muscle signaling. Therefore, it is very unlikely that the 1$\mathrm{hr}$ time difference is going to alter skeletal muscle signaling. The LLC model is not an orthotopic model of lung cancer. There are potential alternative approaches to examining lung cancer in pre-clinical models however, orthotopic models of lung cancer are commonly completed in Balb/c mice or immunodeficient mice, which does not allow for examination of muscle-specific inducible models or for the normal immune response. Lastly, some transgenic models of cancer cachexia utilize tamoxifen administration, a tumor inducer, and would be a significant confounder when using to elicit a musclespecific knockout (Kellar et al., 2015; Safari \& Meuwissen, 2015). Importantly, the LLC model is commonly used due to its tumorigenic properties, responsiveness to chemotherapy, and ability to metastasize to the lungs and liver (Kellar et al., 2015), thus allowing us to use a well characterized model to examine cancer cachexia progression.

\section{Conclusion}

In conclusion, feeding has regulation to increase muscle Akt/mTORC1 and protein synthesis, while lowering AMPK signaling. We report the novel finding that precachectic LLC tumor-bearing do have the capacity to elicit an increase in muscle Akt/mTORC1 signaling and protein synthesis. Importantly, LLC tumor-bearing mice have overall suppressed muscle Akt/mTORC1 signaling and protein synthesis in the fast and fed condition. Lastly, muscle-specific AMPK loss has regulation on the fasting suppression of muscle mTORC1 and protein synthesis in pre-cachectic muscle. Future studies should examine if cachectic muscle has the capacity to increase muscle Akt/mTORC1 signaling and protein synthesis. Additionally, further work is required to determine why muscle AMPK loss in combination with feeding did not restore impaired muscle protein synthesis in pre-cachectic tumor-bearing mice. 


\section{CHAPTER 4. EXAMINATION OF WHETHER MUSCLE GP130 SIGNALING AFFECTS THE FEEDING REGULATION OF AMPK DURING CANCER CACHEXIA}

\section{Introduction}

Inflammation has been implicated as a key mediator of cancer-induced cachexia leading to chronic elevation of skeletal muscle STAT3 and MAPK activity (Deans \& Wigmore, 2005). Glycoprotein-130 (gp130) is a regulatory point of interaction between systemic inflammation and JAK/STAT and MAPK muscle signaling (White \& Stephens, 2011). We have previously reported muscle gp130 loss prevented LLC induced muscle wasting and restored STAT3 and MAPK signaling (Puppa, Gao, et al., 2014).

Interestingly, the LLC model has known disruptions in mitochondrial function prior to muscle wasting (Brown et al., 2017). Additionally, disruptions to the muscle mitochondria are known to induce AMPK, thereby accelerating muscle wasting (VanderVeen et al., 2017). Given that muscle gp130 can regulate mitochondrial quality control (Hardee, Counts, et al., 2018), identifying if these are linked to cachexia's disruption of muscle protein synthesis are warranted.

While it is well established that muscle protein breakdown is activated during wasting, whether suppressed protein synthesis regulation contributes to muscle mass loss during cachexia is less known. To date, only a few studies have examined muscle protein synthesis in cancer patients (Dworzak et al., 1998; Emery et al., 1984; van Dijk et al., 2015; van Dijk et al., 2019). While there are only a few human studies, it has been concluded that changes in both basal and postprandial muscle protein synthesis and breakdown rates contribute to the muscle wasting observed in cachectic cancer patients (Brook et al., 2017; Horstman et al., 2016). A majority of our understanding of protein synthesis during cachexia is examined in murine models, but reports have also shown protein synthesis rates reduced in cachectic patients (Dworzak et al., 1998). We have routinely shown suppressed protein synthesis during the progression of cachexia in Apc $\mathrm{Min} /+$ mice, and this suppression corresponded to a reduction in muscle protein mTORC1 and IGF1 (White, Baynes, et al., 2011). This early suppression of protein synthesis during cachexia did not correspond to an initial change in AMPK, since AMPK activity does not increase till late-stage cachexia under basal conditions (White, Baynes, et al., 2011).

Interestingly, IL-6 receptor antibody was sufficient to attenuate muscle mass loss and AMPK signaling, however IL-6 antibody was not sufficient to restore protein synthesis or mTORC1 (White, Baynes, et al., 2011). Additionally, we have examined a role for systemic IL-6 in suppressing protein synthesis during cachexia (White et al., 2013). Cytokine dosing is commonly used in cell culture to provide a high inflammatory environment. We have previously shown that IL-6 exposure has the capacity to decrease C2C12 myotube diameter and suppress mTORC1 signaling, and when AMPK is blocked the IL-6 suppression of protein synthesis is relieved (White et al., 2013). In the LLC tumor model, downstream targets of mTORC1; 4EBP-1 and rpS6, are suppressed during cachexia (Puppa, Gao, et al., 2014). Interestingly, gp130 loss did not improve the 
suppressed mTORC1 signaling, but did prevent muscle wasting (Puppa, Gao, et al., 2014). Furthermore, LLC tumor derived media on C2C12 myotubes suppressed protein synthesis which coincided with STAT3 and AMPK phosphorylation (Gao \& Carson, 2016). AMPK, but not gp130 inhibition rescued protein synthesis suppression. Collectively, these studies highlight that suppressed muscle protein synthesis and mTORC1 signaling is linked to IL-6/STAT3, but alone is not sufficient to restore protein synthesis during cachexia progression.

Several studies have implicated the role of gp130 signaling on preventing and/or treating cancer induced cachexia. First, skeletal muscle-specific STAT loss prevented LLC induced muscle wasting and grip strength loss, without altering tumor mass (Silva et al., 2015). Interestingly, in a genetic model of cancer induced cachexia ( $A p c^{\mathrm{Min} /+}$ mice), IL-6 receptor antibody preserved some body weight and muscle mass loss (White, Baynes, et al., 2011). Furthermore, IL-6 receptor antibody was sufficient in reducing STAT3 and AMPK signaling without rescuing the suppression of mTORC1. We have previously administered PDTC (anti-inflammatory) to mice injected with LLC cells, while muscle STAT3 was reduced, muscle mass was not preserved (Puppa, Gao, et al., 2014). Lastly, IL-6 induced mitochondrial dysfunction and fatigue requires skeletal muscle gp130 (VanderVeen et al., 2019). Interestingly, cachexia has known disruptions in mitochondrial function prior to muscle wasting (Brown et al., 2017) and theses disruptions to the muscle mitochondria are known to induce AMPK, thereby accelerating muscle wasting (VanderVeen et al., 2017). Given that muscle gp130 can regulate mitochondrial quality control (Hardee, Counts, et al., 2018), whether gp130 is linked to cachexia's disruption of protein synthesis by feeding is warranted. Therefore, the purpose of aim 2 examined if muscle gp130 signaling regulates the feeding regulation of AMPK during cancer cachexia. We hypothesized that gp130 signaling would be required for the feeding regulation of AMPK during cancer cachexia and would be early event in cancer cachexia.

\title{
Methods
}

\begin{abstract}
Animals
To examine the regulation of gp130 loss in tumor-bearing mice, gp130 knockout mice and floxed controls were generated (see next section for details). Mice were kept on a 12:12h light/dark cycle beginning at 6:00AM and were given rodent chow ad libitum (Harlan Teklad Rodent Diet, \#8604, Harlan, Indianapolis, IN, USA). Between 17-18wks of age, male gp130 knockout or floxed controls were either injected with PBS or LLC cells then sacrificed 4 weeks later. All mice were fasted for $12 \mathrm{hrs}$ during the dark cycle. At the start of the light cycle, mice were either given ad libitum access to a food pellet (fed) or sacrificed at the end of fast (fast).
\end{abstract}




\section{Generation of gp130 skeletal muscle deletion}

To generate a skeletal muscle-specific knockout of gp130, mice that contained individually floxed alleles for gp130 were a kind gift from Dr. Colin Stewart's Laboratory (Laboratory of Cancer and Development Biology, National Cancer Institute, U.S. National Institutes of Health (NIH), Frederick, MD, USA) in collaboration with Dr. Lother Hennighausen (Laboratory of Genetics and Physiology, National Institute of Diabetes and Digestive and Kidney Disease, NIH, Bethesda, MD) (Zhao et al., 2004). The skeletal actin (HSA) promoter-driven expression of a Cre recombinase flanked by mutated estrogen receptors (HSA- MCM) (McCarthy et al., 2012). Tamoxifen inducible Mer Cre Mer driven by a human skeletal actin promoter (HSA) mouse were purchased from Jackson Laboratories (Bar Harbor, ME, USA). Male tamoxifen-inducible skeletal muscle gp130 (KO) knockout mice and floxed (Flox) controls were produced. Finally, the gp130 HSA Cre (gp130 KO) mice or gp130 (floxed control) mice were injected with PBS or LLC. At approximately 10-11 weeks of age, all gp130 KO and floxed control mice received tamoxifen injection (I.P. $2 \mathrm{mg}$ ) once daily for 5 consecutive days (Harfmann et al., 2016), and then underwent a 2-week washout period before PBS or LLC injections (Figure 4-1).

\section{Lewis lung carcinoma cell inoculation}

Between 13-14 weeks of age, gp130 mice were injected with either phosphate buffered saline (PBS) or $1 \times 10^{6}$ Lewis lung carcinoma (LLC) cells subcutaneously in the right flank under anesthesia for less than 3 minutes (Puppa, Gao, et al., 2014). A total of 38 mice were injected with LLC cells and 32 mice were injected with PBS. Ninety-two percent of the LLC injected mice reached the studies endpoint. A mouse reached the studies endpoint if the following criteria were met (1) mouse reached 30 days post tumor inoculation, (2) $>20 \%$ body weight loss from day 10 after day 25 , or (3) had a tumor $>3 \mathrm{~cm}$ in width or length after day 25 . Once an endpoint was achieved, the mouse was prepared for tissue collection and euthanized within 24 hrs. At day 22-23, 4 mice had ulcerated tumors and was not included in the analysis. To be included in the study, mice needed to achieve at least 25 days post tumor inoculation. Therefore, a total of 35 male LLC injected mice were used in these experiments. Tumor volume and body weight were measured every 5 days to calculate tumor growth. Tumor volume was calculated by the same investigator and caliper using the following equation: $1 / 2$ (width ${ }^{2}$ xlength) (Jensen et al., 2008).

\section{Cell culture experiments}

All cells were purchased through ATCC (Manassas, VA) and after pass \#5 and within the 10 passages. Murine C2C12 myoblasts (CRL-1772) or Lewis lung carcinoma (LLC; CRL-1462) cells were maintained at $37^{\circ} \mathrm{C}, 5 \% \mathrm{CO} 2$ in Growth Media (GM): Dulbecco's Modified Eagle Media (DMEM; Gibco \#11995-065) supplemented with 10\% FBS, $50 \mathrm{U} / \mathrm{ml}$ penicillin, and $50 \mu \mathrm{g} / \mathrm{ml}$ streptomycin. 
A.

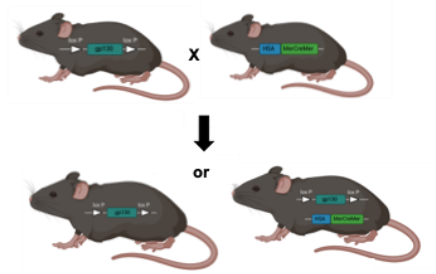

B.

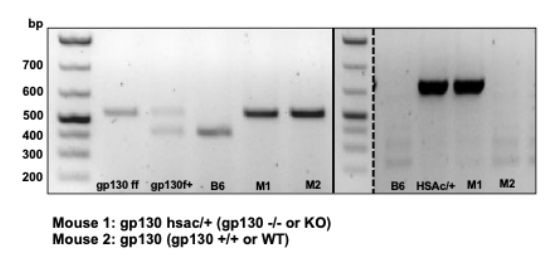

c.

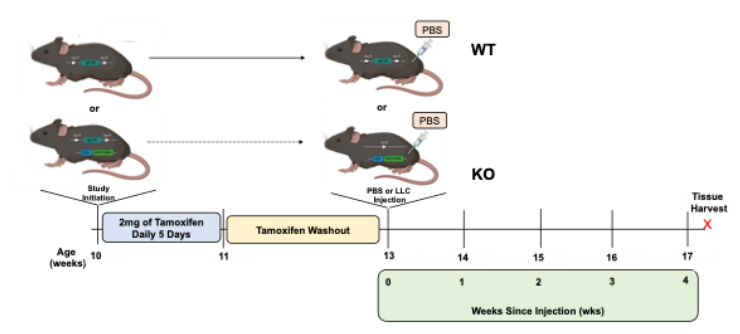

D.

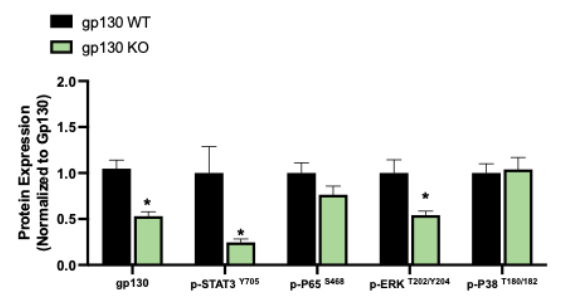

Figure 4-1. Muscle-specific loss of gp130 in male mice

Notes: Data are presented as mean $\pm \mathrm{SEM}$. Male C57B1/J mice were used from UTHSC breeding colony. A. Breeding schematic to produce gp $130+/+$ with and without HSAmer-cre-mer. Created with BioRender.com. B. Study Design. C. Example of tail snip confirmation of animal genotype. D. Muscle-specific loss of gp130 and associated downstream signaling. Phospho- proteins were normalized to total protein. Abbreviations: gp130: glycoprotein 130, WT: Wildtype - gp130 +/+ (WT), KO: Knockout - gp130 -/- (KO), PBS: phosphate buffered saline, LLC: Lewis lung carcinoma, mg: milligrams 


\section{C2C12 myotube differentiation and conditioned media collection}

C2C12 myoblasts were seeded on type I collagen-coated plates at a density of 9.0x $10^{4}$ cells per well (6-well plate) in Growth Media (GM): Dulbecco's Modified Eagle Media (DMEM; Gibco \#11995-065) supplemented with 10\% FBS, 50U/ml penicillin, and $50 \mu \mathrm{g} / \mathrm{ml}$ streptomycin. To induce myoblast differentiation, cells were rinsed with PBS and switched to differentiation media (DM): DMEM supplemented with $2 \%$ heatinactivated horse serum, $50 \mathrm{U} / \mathrm{ml}$ penicillin, and $50 \mu \mathrm{g} / \mathrm{ml}$ streptomycin to form myotubes. Media was replenished every $48 \mathrm{~h}$, and experiments were performed starting at day 5 of differentiation when multinucleated contractile myotubes are present.

LLC cells, a murine model that promotes cachexia in mice, was cultured as described above. Briefly, $2 \times 10^{6}$ cells were seeded in $100 \mathrm{~mm}$ tissue culture-treated plates in GM. Tumor cell CM was collected at $\sim 90 \%$ confluence $48 \mathrm{~h}$ post cell seeding and spun down at $4000 \mathrm{rpm}$ for $5 \mathrm{~min}$ to remove cell debris. Cells on the plate were pelleted and counted via trypan blue exclusion test to ensure an equivalent number of cells on the plate, with a final density averaging from $7.0-9.0 \times 10^{6}$ cells per culture dish. CM was stored for one-time use in aliquots at $-20^{\circ} \mathrm{C}$ and used within 2 months, then thawed in a warm water bath at the time of the experiment. GM with no cells was used as a media control.

\section{Treatment of myotubes}

At the time of the experiment, GM control or LLC CM is diluted with 50\% serum-free DMEM for a final serum concentration of 5\% FBS. 50\% CM was chosen as it has been shown to produce significant myotube atrophy (Pin et al., 2018; Zhong et al., 2019). It should be noted that myotubes were differentiated up to day 5 in $2 \%$ Horse Serum, thus upon CM addition, myotubes were switched to a higher serum environment, which can induce myotube hypertrophy (von Walden et al., 2016). CM was replenished after $24 \mathrm{hr}$. In a separate experiment, differentiated myotubes at day 5 were treated with $50 \%$ GM or LLC CM (LLC + GM) for $48 \mathrm{hrs}$. After $48 \mathrm{hrs,} \mathrm{media} \mathrm{was} \mathrm{removed,} \mathrm{and} \mathrm{cells}$ were placed in minimal essential media to quiesce the myotubes. After 60 minutes, $20 \mathrm{nM}$ of insulin was dosed on the cells for 60 minutes and then cells were scraped. Differentiated C2C12 myotubes were treated with $10 \mathrm{ng} / \mathrm{ml}$ of IL-6 (Sigma-Aldrich) or LIF (Thermo Scientific, Waltham, MA) for $24 \mathrm{hrs}$. After $24 \mathrm{hrs}$, media was removed, and cells were placed in minimal essential media to quiesce the myotubes. After 60 minutes, $20 \mathrm{nM}$ of insulin was dosed on the cells for 60 minutes and then cells were scraped.

\section{Myotube diameter}

Myotube diameter was quantified as previously described (Gao \& Carson, 2016) with the following modifications. $\mathrm{C} 2 \mathrm{C} 12$ myotube diameter was quantified using ImageJ software (National Institutes of Health, Bethesda, MD). Digital images were captured at 20X objective brightfield. Five non-overlapping images were captured within each well, 
and three images were randomly chosen for analysis. The analysis used unmodified tiff images accessed in NIH ImageJ software. A blinded investigator randomly took diameter measurements of 6 myotubes per image based on pre-set inclusion/exclusion criteria: elongated structure with distinct membrane outlines, little to no cellular debris, no branching points. The average diameter per myotube was calculated as the mean of 8 measurements taken along the myotube length.

\section{Western blot}

Western blot analysis was performed as previously described (Hardee et al., 2014) and extensive methodology can be found in extended methods section. Briefly, frozen red and white gastrocnemius muscle was homogenized in lysis buffer and protein concentration was determined by the Bradford method. Crude gastrocnemius muscle homogenates were fractionated on 8-12\% SDS-polyacrylamide gels and transferred to PVDF membranes. Membranes were stained with Ponceau red to verify equal loading and transfer. Membranes were then blocked at room temperature for $1 \mathrm{hr}$. in $5 \%$ non-fat milk or 5\% BSA-Tris-buffered saline with $0.1 \%$ Tween-20 (TBST). Commercially available phosphorylated and total protein primary antibodies for rpS6, AKT, AMPK, STAT3, ERK, P65, P38, gp130 were raised in rabbit. RpS6, AKT, AMPK, STAT3, ERK, P65, and P38 phosphorylation antibodies were expressed relative to total protein on the same gel and quantified as phosphorylation to total ratio. Commercially available total protein primary antibodies for MuRF-1, Atrogin-1, PGC1 $\alpha$, OPA1, and FIS1 were raised in rabbit. Puromycin incorporation was purchased from Millipore and raised in mouse. Briefly, primary antibodies were incubated overnight in 5\% TBST milk or 5\% TBST BSA. Membranes were then incubated in 5\% milk-TBST containing anti-rabbit or antimouse IgG horseradish-peroxidase conjugated secondary antibody purchased from cell signaling for $1 \mathrm{~h}$ at room temperature. Enhanced chemiluminescence (ECL) (GE Healthcare Life Sciences, Piscataway, NJ) was used to visualize the antibody-antigen interactions. Immunoblot images were collected using a digital imager (Invitrogen iBright, Waltham, MA) and quantified by densitometry using imaging software (Image J; $\mathrm{NIH}$ ). Please see end of document for details of fcatalogue numbers, companies, dilutions, incubation durations, and secondary substrate (Figure B-1).

\section{Statistical analysis}

Pre-planned unpaired T-Test were used to compare PBS and LLC mice. Preplanned unpaired T-Test were used to compare to validate the feeding response and knockout signaling Two-way (Tumor x Feeding) (Feeding x Genotype) ANOVAs were used, and Tukey's post-hoc analysis was used when appropriate. Prism 8 (GraphPad) was used for statistical analysis. All results are reported as means \pm standard error of mean (SEM). Statistical analysis was performed using GraphPad (Prism 8 for Mac OS X, La Jolla, Ca). Level of significance for all measures was set at $p \leq 0.05$. 


\section{Results}

\section{Gp130 loss validation}

Skeletal muscle-specific knockout of gp130, mice that contained individually floxed alleles for gp130 were a kind gift from Dr. Colin Stewart's Laboratory (Laboratory of Cancer and Development Biology, National Cancer Institute, U.S. National Institutes of Health (NIH), Frederick, MD, USA) in collaboration with Dr. Lother Hennighausen (Laboratory of Genetics and Physiology, National Institute of Diabetes and Digestive and Kidney Disease, NIH, Bethesda, MD) (Zhao et al., 2004). Male tamoxifen-inducible skeletal muscle gp130 (KO) knockout mice and floxed (Flox) controls were produced (Figure 4-1). Genotyping was confirmed by tail snips after 21 days neonatal (Figure 4-1). Around 10 weeks of age, mice were given tamoxifen injection once a day for 5 consecutive days to bring the LoxP-MerCreMer bound binding sites together to remove the floxed genes of interest. After a two-week washout period, mice were injected with either phosphate buffered saline (PBS) or Lewis lung carcinoma (LLC) cells and monitored for 4 weeks (Figure 4-1C). To validate gp130 loss and associated signaling, white gastrocnemius muscle was used. Gp130 KO mice have reduced gp130 (-47\%), STAT3 (-83\%), and ERK (-46\%) without changes to P65 or P38 identifying the specificity of gp130 loss (Figure 4-1).

\section{Effect of gp130 loss on skeletal muscle signaling in non-tumor-bearing mice}

Importantly, there were no differences in body weight over time, skeletal muscle mass (gastrocnemius or hindlimb muscle mass), eWAT mass, or tibia length in gp130 WT mice compared to gp130 KO mice (Table 4-1). We highlight the ability to decrease gp130 and associated signaling in skeletal muscle, which does not alter tissue mass and body size in PBS treated male mice. To understand how gp130 loss alters skeletal muscle signaling in tumor-bearing mice, we first need to determine what gp130 loss did to skeletal muscle signaling in non-tumor-bearing male mice in the fast condition. All mice were sacrificed at the start of the dark cycle following an overnight 12-hr fast. It is well known that gp130 can disrupt mitochondrial quality control. Therefore, we examined markers of mitochondrial quality control in white gastrocnemius muscle (Figure B-7). PGC1 $\alpha$ a marker of mitochondrial biogenesis was not altered by gp130 loss.

Additionally, FIS-1, a marker of mitochondrial fission, were not affected by gp130 loss. Lastly, mitochondrial complex proteins (Figure B-7) were not altered by gp130 loss. Gp130 associated signaling has known regulation on mTORC1 signaling, therefore, we examined if gp130 plays a role in basal skeletal muscle anabolic signaling (Figure B-7). Interestingly, gp130 loss did not alter AMPK, ULK, E3 ligases (MuRF-1 or Atrogin-1), AKT, rpS6 or protein synthesis. Taken together, we show that following a 12-hr fast, gp130 loss has regulation on immediate downstream targets, but does not regulate mitochondrial quality control, AMPK, or mTORC1 signaling in white gastrocnemius muscle. 
Table 4-1. Muscle-specific gp130 loss on non-tumor-bearing mice animal characteristics

\begin{tabular}{lccc}
\hline Characteristics & gp130 WT & gp130 KO & \\
N=16 & N=15 & p-Value \\
\hline BW Pre-tamoxifen (g) & $24.2(0.4)$ & $24.4(0.5)$ & 0.488 \\
BW Post-tamoxifen (g) & $24.9(0.3)$ & $24.9(0.5)$ & 0.564 \\
BW Pre-injection (g) & $26.9(0.3)$ & $26.5(0.6)$ & 0.888 \\
BW d10 (g) & $28.2(0.4)$ & $27.2(0.6)$ & 0.338 \\
BW End of Study (g) & $29.1(0.4)$ & $28.7(0.7)$ & 0.964 \\
BW $\Delta$ During Tamoxifen (\%) & $2.9(3.1)$ & $2.4(0.9)$ & 0.686 \\
BW D During Treatment (\%) & $7.5(1.3)$ & $8.4(1.1)$ & 0.583 \\
BW $\Delta$ d10 (\%) & $3.0(0.9)$ & $5.6(1.2)$ & 0.072 \\
HMM (mg) & $204(4)$ & $208(4)$ & 0.476 \\
eWAT (mg) & $340(32)$ & $437(37)$ & 0.055 \\
Testes (mg) & $187(4)$ & $188(6)$ & 0.871 \\
Heart (mg) & $161(5)$ & $147(5)$ & 0.065 \\
Spleen (mg) & $80(4)$ & $73(3)$ & 0.239 \\
Tibia Length (mm) & $17.4(0.1)$ & $17.3(0.1)$ & 0.280 \\
\hline
\end{tabular}

Notes: Data are presented as mean \pm SEM. Abbreviations: gp130: glycoprotein 130, BW: body weight, mg: milligrams, eWAT: epididymal white adipose tissue, PBS: phosphate buffered saline, +/+: wild-type mouse, -/-: knockdown mouse, mm: millimeter, g: grams, and HMM: Hindlimb muscle mass. Unpaired T-Test were used to compare WT and KO. $\mathrm{p}<0.05$. 


\section{Validation of muscle-specific gp130 loss in LLC tumor-bearing mice}

To validate the efficacy of knocking out gp130 and associated signaling in LLC tumor-bearing mice. Tail snips were conducted at neonatal 21 days and mice were determined as having the double floxing of gp130 with or without the presence of hsac/+. After tamoxifen washout, mice were injected with either PBS or LLC cells and sacrificed 4-weeks later (Figure 4-2). We first validated all mice by showing the knockdown expression of gp130 in PBS and LLC gp130 WT and gp130 KO male mice (Figure 4-2B). Importantly, gp130 loss in LLC tumor-bearing mice was sufficient to reduce STAT3, ERK, and P65 (Figure 4-2).

\section{Muscle-specific gp130 loss in LLC tumor-bearing male mice does not alter tumor growth, tumor mass, or indices of cachexia}

Tumor volume increased from days 10-25 in LLC gp130 WT and KO mice $(\mathrm{p}<0.001)$ with no difference between WT and KO mice $(\mathrm{p}=0.829)$ (Figure 4-3). There were no differences in tumor growth rate between gp130 WT and KO LLC mice $(\mathrm{p}=0.859$; Figure 4-3). There were no differences in tumor mass between gp130 WT and KO LLC mice ( $p=0.880$; Figure 4-3C). There was a main effect of time $(p<0.001)$ for body weight during the study to increase at each time point (Table 4-2). There was a main effect for LLC mice to have greater body weight change from day $10(p<0.001)$, without differences between gp130 WT and KO mice $(p=0.713)$. When accounting for tumor mass, there was a main effect for LLC mice to have decreased body weight at the end of the study $(\mathrm{p}<0.001)$, without differences between gp130 WT and KO mice $(\mathrm{p}=0.897)$. There was a main effect for LLC mice to have decreased gastrocnemius mass $(p<0.001)$, without differences between WT and KO mice $(p=0.756)$. There was an interaction for eWAT mass (Figure 4-3); there were no differences within each genotype of PBS or LLC mice. LLC WT mice had decreased eWAT mass compared to PBS KO. LLC KO had decreased eWAT from both PBS groups.

\section{Effect of gp130 loss on AMPK/Akt/mTORC1 signaling and protein synthesis in LLC tumor-bearing male mice}

We examined the effect of gp130 loss in the fasting condition in PBS and LLC mice (Figure 4-4). Gp130 loss in white gastrocnemius muscle suppressed AMPK in LLC tumor-bearing mice (Figure 4-4). Interestingly, there was a main effect of gp130 loss to reduce ULK1 (Figure 4-4). Gp130 loss was not sufficient to lower elevated Atrogin-1 in LLC tumor-bearing mice (Figure 4-4). Interestingly, gp130 loss was not sufficient to improve the basal suppression of muscle protein synthesis in LLC tumor-bearing mice (Figure 4-5). There was a main effect for LLC to have reduced rpS6 and a main effect for gp130 loss to further reduce rpS6 (Figure 4-5). Lastly, there was a main effect for AKT to be reduced in LLC (Figure 4-5). Taken together, we highlight that gp130 loss was sufficient to reduce AMPK without improving suppressed protein synthesis or mTORC1 signaling in LLC tumor-bearing mice. 
A.

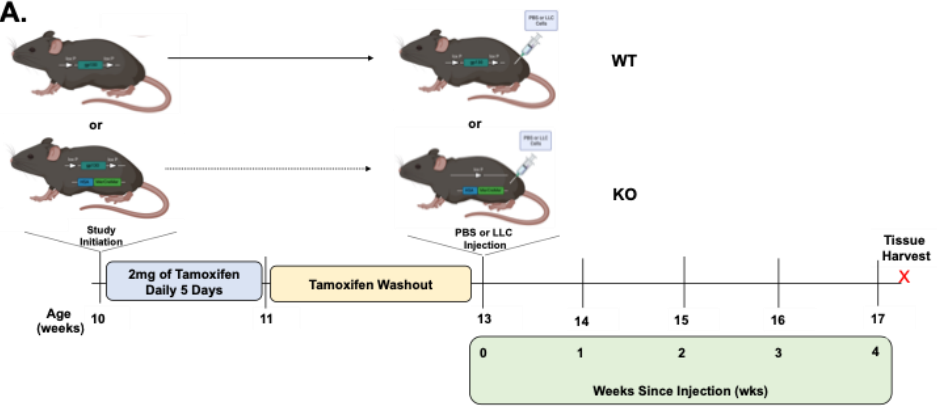

B.

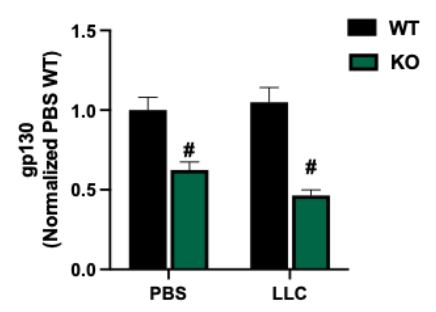

C.

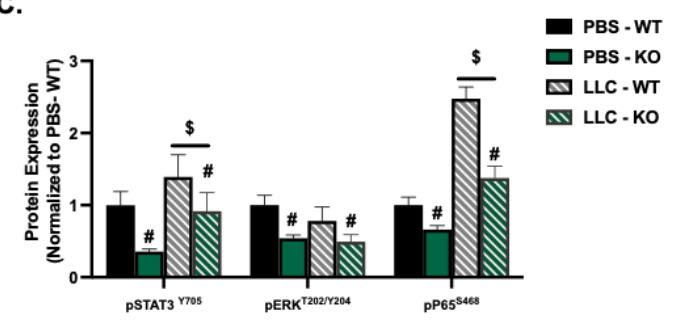

Figure 4-2. Validation of muscle gp130 knockdown and associated downstream signaling in LLC tumor-bearing male mice

Notes: Data are presented as mean $\pm \mathrm{SEM}$. Male C57B1/J mice were used from UTHSC breeding colony. A. Study Design. B. Validation of muscle-specific knockdown expression of gp130 in PBS and LLC mice. C. Validation of gp130 knockdown in KO PBS and LLC male mice. White gastrocnemius muscle was used for analysis. Created with BioRender.com. Phospho- proteins were normalized to total protein. Abbreviations: gp130: glycoprotein 130, WT: Wildtype - gp130 +/+ (WT), KO: Knockout - gp130 -/(KO), PBS: phosphate buffered saline, LLC: Lewis lung carcinoma, mg: milligrams. \# Main effect of KO, \$ Main effect of LLC. N=9-16 per group. 
A.

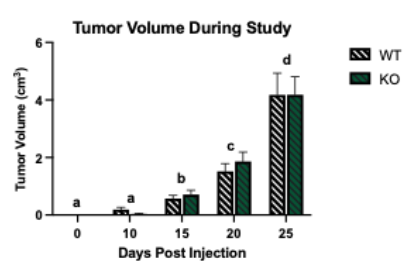

B.

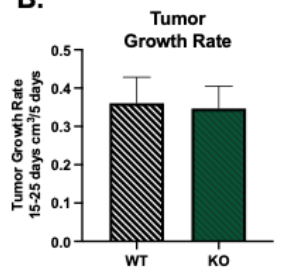

c.

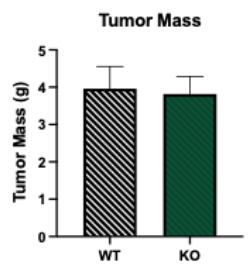

D.

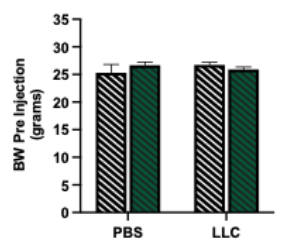

F.

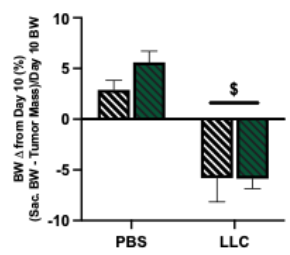

E.

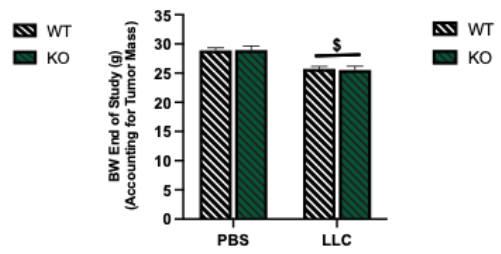

G.
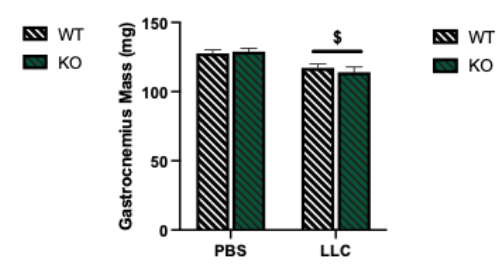

H.

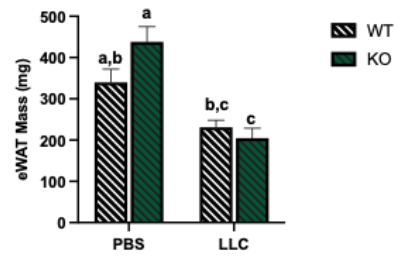

Figure 4-3. Muscle-specific loss of gp130 in LLC tumor-bearing male mice does not alter tumor growth, tumor mass, or indices of cachexia

Notes: Data are presented as mean $\pm \mathrm{SEM}$. A. Study design. male C57B1/6J mice were injected with PBS or LLC cells between 10-11 wks. of age. Tumor volume and body weight were taken every 5 days until sacrifice (28-30 days post tumor inoculation). B.Tumor growth rate from days 15-25. C. Tumor Mass at sacrifice. D. Body weight preinjection of PBS or LLC. E. Body weight end of study accounting to tumor mass. F.Body weight is calculated as the sacrifice body weight minus the tumor mass divided by day 10 body weight. G. Gastrocnemius muscle mass at sacrifice. H. eWAT mass at sacrifice. Phospho- proteins were normalized to total protein. Abbreviations: BW: body weight, mg: milligrams, PBS: phosphate buffered saline, LLC: Lewis lung carcinoma, g: grams. Unpaired t-test were used to compare PBS to LLC. Two-way (Genotype $\mathrm{x}$ LLC) ANOVAs were used. Different letters note differences between time points within WT and KO mice). \$ Main effect of LLC. 
Table 4-2. Effect of muscle-specific gp130 loss on animal characteristics in LLC tumor-bearing male mice

\begin{tabular}{|c|c|c|c|c|}
\hline \multirow[b]{2}{*}{ Characteristics } & \multicolumn{2}{|c|}{ PBS } & \multicolumn{2}{|c|}{ LLC } \\
\hline & $\begin{array}{c}\text { WT } \\
\mathrm{N}=16\end{array}$ & $\begin{array}{c}\text { KO } \\
\mathrm{N}=15\end{array}$ & $\begin{array}{c}\text { WT } \\
\mathrm{N}=16\end{array}$ & $\begin{array}{c}\mathrm{KO} \\
\mathrm{N}=18\end{array}$ \\
\hline Age Inoculated (wks) & $14.2(0.5)$ & $14.1(0.3)$ & $14.9(0.4)$ & $13.9(0.4)$ \\
\hline BW Pre-tamoxifen (g) & $24.2(0.4)$ & $24.4(0.5)$ & $23.7(0.6)$ & $23.5(0.4)$ \\
\hline BW Post-tamoxifen (g) & $24.9(0.3)$ & $24.9(0.5)$ & $25.2(0.3)$ & $24.3(0.4)$ \\
\hline Days in Study (days) & $26.8(0.5)$ & $26.1(0.3)$ & $27.1(0.3)$ & $26.0(0.4)$ \\
\hline BW Pre-inoculation (g) & $26.9(0.3)$ & $26.5(0.6)$ & $26.7(0.5)$ & $25.9(0.4)$ \\
\hline BW d10 (g) & $28.2(0.4)$ & $27.2(0.6)$ & $27.4(0.5)$ & $27.1(0.6)$ \\
\hline BW End of Study (g) & $29.1(0.4)$ & $28.7(0.7)$ & $29.7(0.5)$ & $29.4(0.7)$ \\
\hline Tumor Weight (TW) (g) & - & - & $4.0(0.6)$ & $3.8(0.5)$ \\
\hline $\mathrm{BW}-\mathrm{TW}(\mathrm{g})$ & $29.1(0.4)$ & $28.7(0.7)$ & $25.7(0.4)$ & $25.6(0.6)$ \\
\hline $\mathrm{BW} \Delta$ from $\mathrm{d} 10(\%)$ & $3.0(0.9)$ & $5.6(1.2)$ & $-5.8(2.3)$ & $-5.9(1.0)$ \\
\hline Tumor Volume d15 $\left(\mathrm{cm}^{3}\right)$ & - & - & $0.69(0.01) \mathrm{a}$ & $0.71(0.15) \mathrm{a}$ \\
\hline Tumor Volume d20 $\left(\mathrm{cm}^{3}\right)$ & - & - & $1.52(0.27) \mathrm{b}$ & $1.86(0.33) \mathrm{b}$ \\
\hline Tumor Volume d $25\left(\mathrm{~cm}^{3}\right)$ & - & - & $4.18(0.75) \mathrm{c}$ & $4.18(0.63) \mathrm{c}$ \\
\hline Tumor Growth Rate $\left(\mathrm{cm}^{3} / 5\right.$ days $)$ & - & - & $0.361(0.068)$ & $0.347(0.059)$ \\
\hline Soleus Weight (mg) & $7.3(0.8)$ & $8.1(0.3)$ & $7.0(0.4)$ & $7.3(0.3)$ \\
\hline Gastrocnemius Weight (mg) & $128(3)$ & $128(2)$ & $117(3) \#$ & $114(4) \#$ \\
\hline Tibialis Anterior Weight $(\mathrm{mg})$ & $43(1)$ & $44(1)$ & $41(1) \#$ & $38(2) \#$ \\
\hline HMM (mg) & $205(4)$ & $206(4)$ & $189(5) \#$ & $183(6) \#$ \\
\hline Testes Weight (mg) & $189(4)$ & $186(6)$ & $184(4)$ & $178(40$ \\
\hline Seminal Vesicle Weight (mg) & $215(10)$ & $232(8)$ & 187 (10)\# & 200 (7)\# \\
\hline eWAT (mg) & $341(30) \mathrm{a}, \mathrm{b}$ & 444 (39)a & $231(17) \mathrm{b}, \mathrm{c}$ & $205(24) \mathrm{c}$ \\
\hline Spleen Mass (mg) & $80(4.1)$ & $73(3)$ & $226(23) \#$ & $243(24) \#$ \\
\hline Plasma IL-6 (pg/ml) & $5.0(0.8)$ & $4.7(0.5)$ & 21.7 (2.4)\# & 16.4 (3.3)\# \\
\hline Tibia Length $(\mathrm{mm})$ & $17.4(0.1)$ & $17.3(0.1)$ & $17.2(0.1)$ & $17.2(0.1)$ \\
\hline
\end{tabular}

Notes: Data are presented as Mean \pm SEM. Male C57Bl/J mice were used from UTHSC breeding colony. Sixty-five mice were randomly assigned to either PBS $(\mathrm{N}=31)$ or LLC $(\mathrm{N}=34)$ based on their genotype of gp130 WT or gp130 KO. Then at the time of sacrifice were either fasted or fed. There were no differences within each fast or fed condition of PBS or LLC in either genotype therefore groups are combined here. Abbreviations: SEM: Standard error of the mean, $\mathrm{cm}^{3}$ : centimeters cubed, mg: milligrams, BW: body Weight, TW: tumor weight, $\Delta$ : Change, \%: Percent, LLC: Lewis lung carcinoma, PBS: Phosphate Buffered Saline, mm: millimeters, and HMM: hindlimb muscle mass. Statistical Significance $\mathrm{p}<0.05$. Two-way ANOVA ( 2 genotype $\mathrm{x} 2$ tumor) was used to compare across groups. ME Genotype: ^, ME Tumor: \#. Different letters mean there was an interaction and different from other letters. One-way repeated measures ANOVA was used to compare body weight overtime within each treatment and genotyped. One-way ANOVA was used to compare tumor volume overtime within each genotype group of LLC mice. Different letters mean there was an interaction and different from other letters. 
A.

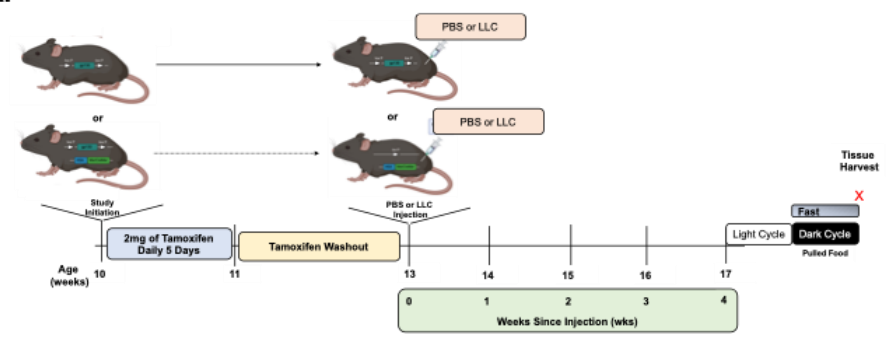

B.

gp130 WT

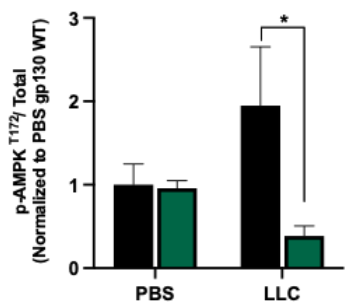

E.

gp130 WT

$\square$ gp130 kо

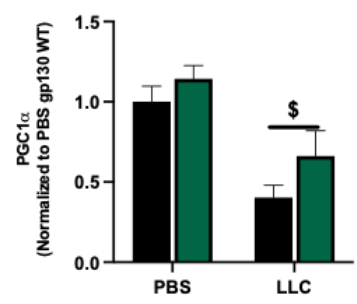

C.

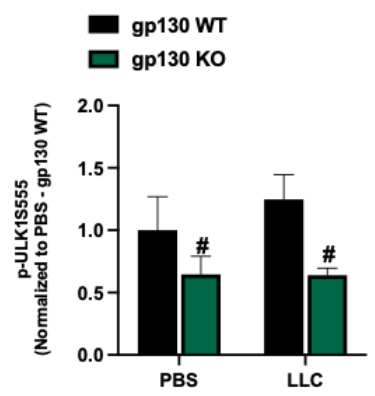

F.

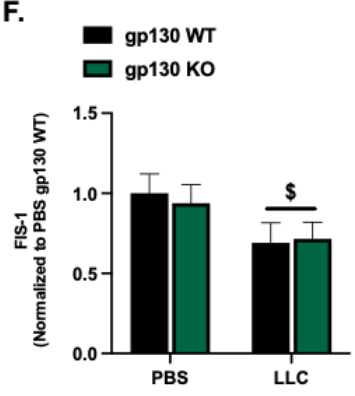

D.

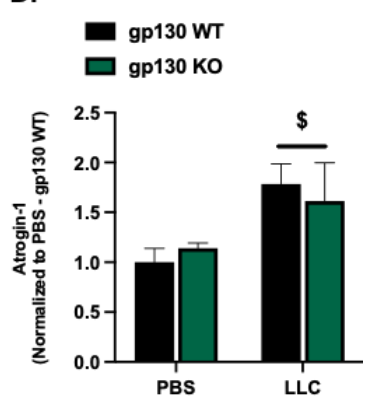

Figure 4-4. Muscle-specific gp130 loss reduces AMPK in LLC tumor-bearing mice

Notes: Data are presented as mean \pm SEM. A. Study Design B. Phospho-AMPK to total ratio in PBS and LLC fasted mice either gp130 WT or gp130 KO. C. Phospho-ULK1 in PBS and LLC fasted mice either gp130 WT or gp130 KO. D. Atrogin-1 in PBS and LLC fasted mice either gp130 WT or gp130 KO. E. PGC1 $\alpha$ protein expression in PBs and LLC fasted mice either gp130 WT or gp130 KO. F. FIS-1 protein expression in PBS and LLC fasted mice either gp130 WT or gp130 KO. Phospho- proteins were normalized to total protein. Abbreviations: PBS: phosphate buffered saline, LLC: Lewis lung carcinoma, g: grams. Two-way ANOVAs (PBS or LLC vs WT or KO) were used. *Groups are different. \# Main effect of KO, \$ Main effect of LLC. 
A.

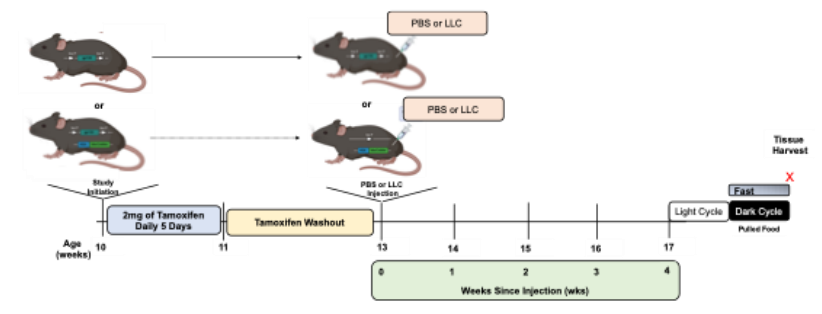

B.

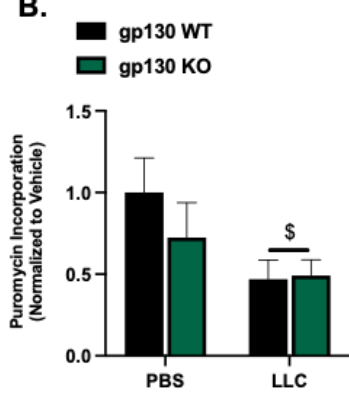

C.

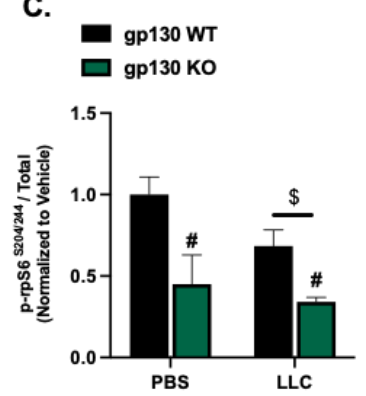

D.

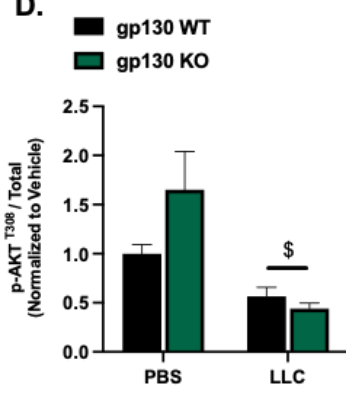

Figure 4-5. Muscle-specific gp130 loss does not rescue suppressed protein synthesis in LLC tumor-bearing mice

Notes: Data are presented as mean \pm SEM. A. Study Design. B. Phospho-AMPK to total ratio in PBS fast and fed gp130 WT and gp130 KO mice. C. Phospho-AMPK to total ratio in LLC fast and fed gp130 WT and gp130 KO mice. Phospho- proteins were normalized to total protein. Abbreviations: PBS: phosphate buffered saline, LLC: Lewis lung carcinoma. Two-way ANOVAs (PBS or LLC vs WT or KO) were used. * Different between groups. 


\section{Effect of muscle-specific gp130 loss on skeletal muscle's feeding regulation of skeletal muscle protein turnover}

Due to different animal cohorts being used because of tamoxifen use, we first validated that there were no differences in gp130 WT and KO mice whole-body response to feeding, therefore these outcomes are combined. Importantly, whole-body response to feeding in PBS and LLC mice was similar to previous aim1 (Table 4-3). We have shown gp130 loss can reduce AMPK in LLC tumor-bearing mice. We next sought to determine if gp130 loss regulates the feeding regulation of AMPK. We report that gp130 loss blunted the reduction in AMPK in PBS mice (Figure 4-6). Feeding was sufficient to lower AMPK in LLC mice; however, this response was not altered in gp130 KO mice (Figure 4-6). Taken together, our results suggest that in pre-cachectic tumor-bearing mice, gp130 loss disrupts the feeding regulation of AMPK. Previously, we have shown gp130 loss does not rescue the suppression of mTORC1 or protein synthesis in LLC tumor-bearing mice. Interestingly, gp130 loss lowered mTORC1 signaling, suggesting some regulation. Therefore, we next sought to determine if gp130 loss regulates the muscle's anabolic response to feeding. In PBS mice, there was a main effect for feeding to increase AKT and main effect for gp130 loss to increase AKT (Figure 4-7). Gp130 loss did not alter the feeding response in LLC tumor-bearing mice (Figure 4-7). Gp130 loss did not alter the feeding induction of $\mathrm{rpS} 6$ or protein synthesis in PBS mice (Figure 4-7). Interestingly, there was a main effect for gp130 loss to reduce rpS6 in LLC mice and a main effect for feeding to increase rpS6. Gp130 loss did not alter the feeding induction of protein synthesis in LLC mice (Figure 4-7). Interestingly, feeding can reduce STAT3 and P65 protein expression in tumor-bearing mice (Figure B-8). Taken together we highlight that the feeding regulation of $\mathrm{mTORC} 1$ signaling and protein synthesis is partially regulated by gp130 in tumor-bearing mice.

\section{Effect of LLC-conditioned media on Akt/mTORC1 response to insulin in C2C12 myotubes}

LLC-conditioned media is commonly used to examine the effects of tumor derived factors on $\mathrm{C} 2 \mathrm{C} 12$ myotubes. LLC conditioned media suppresses myotube diameter and myosin heavy chain-fast (Figure 4-8) compared to growth media control. LLC-conditioned media induced myotube STAT3 and ERK protein expression (Figure 4-8) compared to GM control. Following $48 \mathrm{hrs}$ of conditioned media, media was changed to minimal essential media for 60 minutes, then insulin was given to the cells (Figure 4-9). We report that insulin significantly induced Akt and rpS6 in GM treated myotubes (Figure 4-9). Insulin was sufficient to induce Akt and rpS6 in LLC treated myotubes, however the overall response was lower compared to GM. We next sought to examine if cytokines (tumor derived factors) were inhibiting the overall response (Figure 4-10). Interestingly, neither IL-6 nor Lif were sufficient to suppress the Akt or rpS6 in C2C12 myotubes. IL-6 nor Lif altered the insulin induction of Akt or mTORC1, despite having elevated STAT3. Taken together, these results suggest that IL-6 nor Lif alone are sufficient to inhibit basal Akt/mTORC1 signaling. 
Table 4-3. Whole-body response to feeding in PBS and LLC male mice

\begin{tabular}{|c|c|c|c|c|}
\hline \multirow[b]{2}{*}{ Outcomes } & \multicolumn{2}{|c|}{ PBS } & \multicolumn{2}{|c|}{ LLC } \\
\hline & $\begin{array}{c}\text { Fast } \\
N=14\end{array}$ & $\begin{array}{c}\text { Fed } \\
\mathbf{N}=17\end{array}$ & $\begin{array}{c}\text { Fast } \\
\mathbf{N}=17\end{array}$ & $\begin{array}{c}\text { Fed } \\
\mathbf{N}=17\end{array}$ \\
\hline Pellet Consumption (g) & - & $0.61(0.07)$ & - & $0.49(0.06)$ \\
\hline Blood Glucose (mg/dl) & $100(5)$ & $157(5)^{\wedge}$ & $126(5) \#$ & $192(9)^{\wedge} \#$ \\
\hline Plasma Insulin (ng/mL) & $0.40(0.09)$ & $1.09(0.17)^{\wedge}$ & $0.29(0.04) \#$ & $0.82(0.12)^{\wedge} \#$ \\
\hline Stomach Mass (mg) & 399 (39)a & $801(74) b$ & $375(36) \mathrm{a}$ & $652(58) c$ \\
\hline Empty Stomach (mg) & $166(7)$ & $162(3)$ & $169(10)$ & $143(6)$ \\
\hline Stomach Content (mg) & $241(44)$ & $640(75)^{\wedge}$ & $206(34)$ & $509(56)^{\wedge} \#$ \\
\hline
\end{tabular}

Notes: There were no effects of gp $130 \mathrm{KO}$ on whole-body response to feeding therefore PBS and LLC AMPK WT and AMPK KO mice in the fast or fed condition are combined to show the summary data. Data are presented as Mean (SEM). Abbreviations: mg: milligrams, PBS: phosphate buffered saline, LLC: Lewis lung carcinoma, g: grams, $\mathrm{mg} / \mathrm{dl}$ : milligrams per deciliter, $\mathrm{ng} / \mathrm{mL}$ : nanograms per milliliter, and ME: Main Effect. Unpaired T-test was used to compare PBS and LLC fed pellet consumption. Two-way ANOVAs were used to compare PBS and LLC in the Fast and Fed condition. \#ME of Fed. If an interaction was present; different letters were used to differentiate differences between groups. Statistically significant $p<0.05$. 
A.

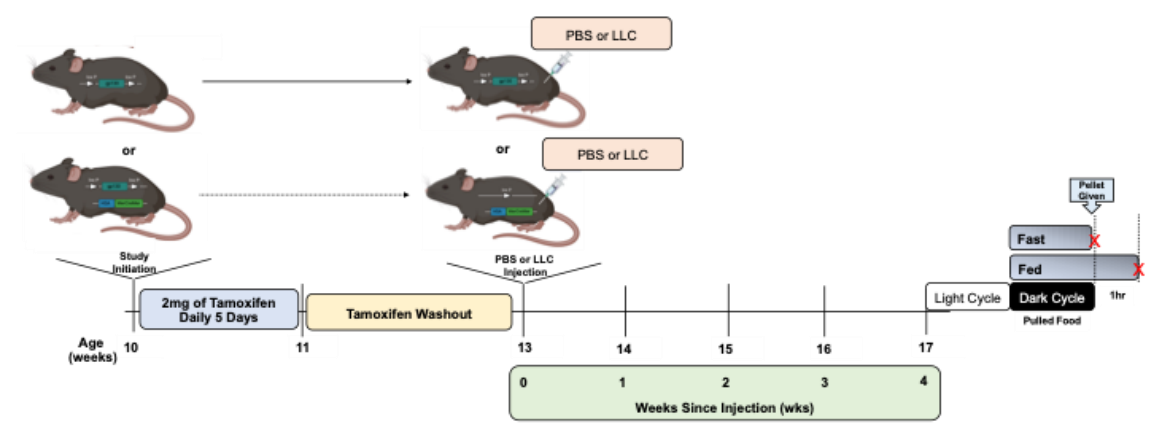

B. PBS Mice

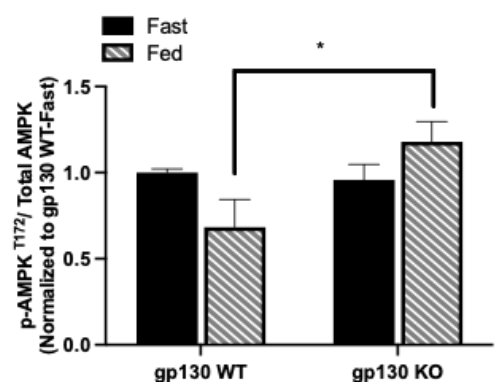

C. LLC Mice

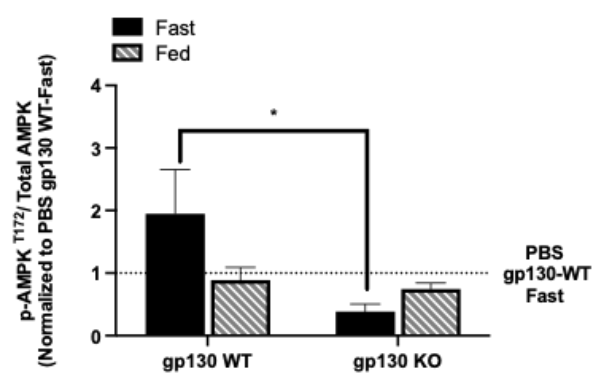

Figure 4-6. Muscle-specific gp130 loss disrupts AMPK's reduction by feeding

Notes: Data are presented as mean \pm SEM. A. Study Design. B. Phospho-AMPK to total ratio in PBS fast and fed gp130 WT and gp130 KO mice. C. Phospho-AMPK to total ratio in LLC fast and fed gp130 WT and gp130 KO mice. Phospho- proteins were normalized to total protein. Abbreviations: PBS: phosphate buffered saline, LLC: Lewis lung carcinoma. Two-way ANOVAs (PBS or LLC vs WT or KO) were used. *-Different between groups. 
A.

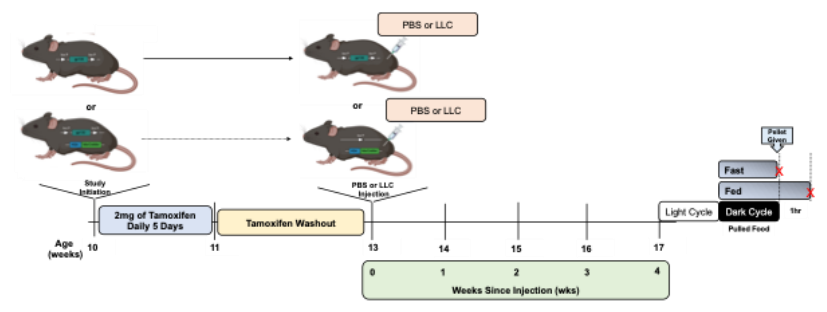

B.

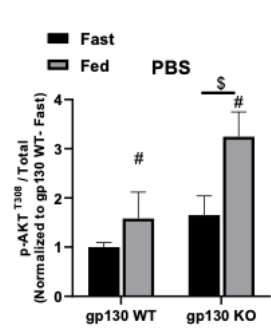

E.

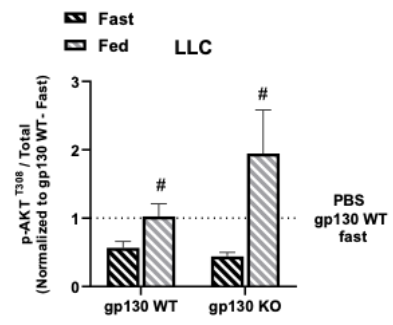

c.

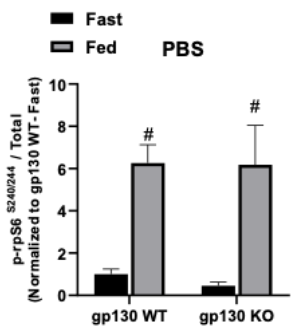

G.

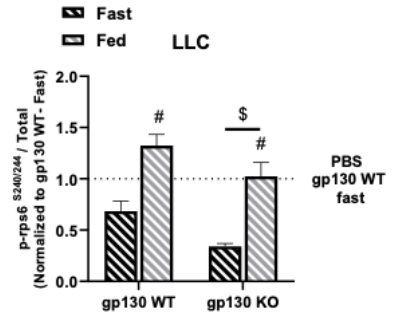

D.

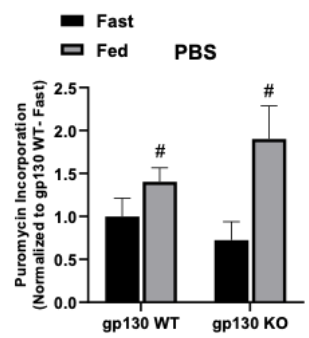

H.

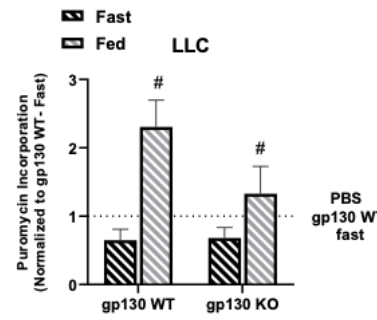

Figure 4-7. Muscle-specific gp130 loss regulation of Akt/mTORC1 signaling and protein synthesis response to feeding in LLC tumor-bearing mice

Notes: Data are presented as mean \pm SEM. A. Study Design. B. Phospho-AKT to total ratio in PBS fast and fed gp130 WT and gp130 KO mice. C. Phospho-rps6 to total ratio in PBS fast and fed gp130 WT and gp130 KO mice. D. Puromycin Incorporation in PBS fast and fed gp130 WT and gp130 KO mice. E. Phospho-AKT to total ratio in LLC fast and fed gp130 WT and gp130 KO mice. G. Phospho-rps6 to total ratio in LLC fast and fed gp130 WT and gp130 KO mice. H. Puromycin Incorporation in LLC fast and fed gp130 WT and gp130 KO mice. LLC mice are normalized to PBS-gp130 WT Fast mice. Statistical analysis was only completed within the LLC mice. Phospho- proteins were normalized to total protein. Abbreviations: PBS: phosphate buffered saline, LLC: Lewis lung carcinoma. Two-way ANOVAs (PBS or LLC vs WT or KO) were used. \# Main effect of Fed, \$ Main effect of gp130 KO. 
A.

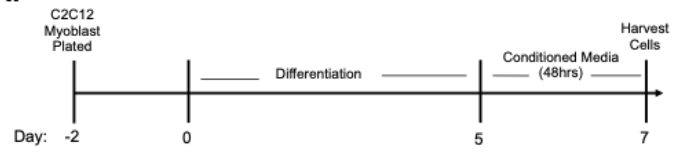

B.

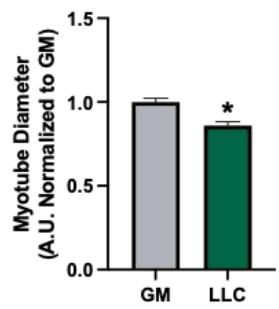

C.

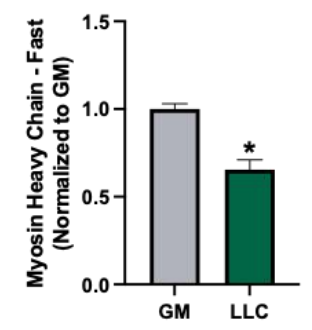

F.

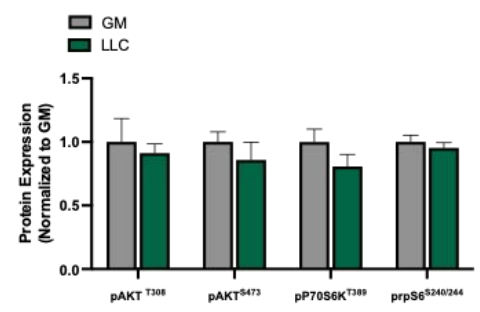

D.

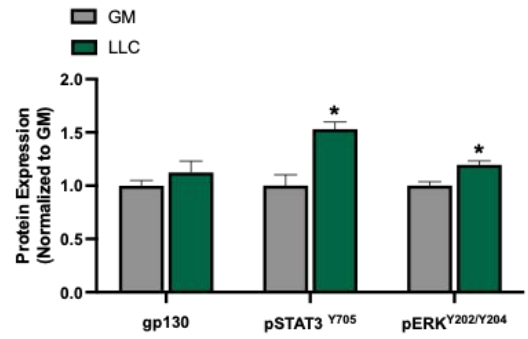

G.

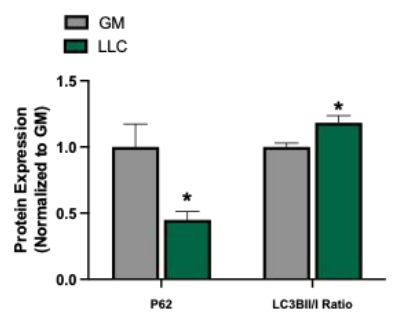

E.

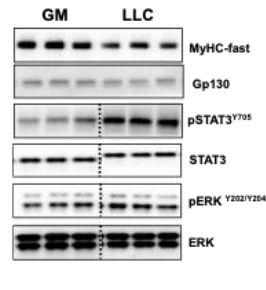

H. см цс

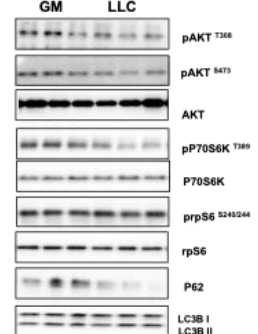

Figure 4-8. LLC conditioned media suppresses myotube growth and induces inflammatory associated signaling in C2C12 myotubes

Notes: Data are presented as mean \pm SEM. A. Study Design. B. Myotube Diameter C.Myosin heavy chain - fast protein expression. D. Protein expression of gp130 and phosphor- to total ratio of STAT3 Y705 and ERK1/2 Y202/Y204. E. Phospho-to total ratio of AKT T308 and S473, rpS6 S240, and P70S6K T389 G. Protein expression of P62 and LC3B II/I ratio. Unpaired T-test were used to compare GM and LLC media.

*Different from GM. $\mathrm{N}=6 / 2$ separate experiments. 
A.

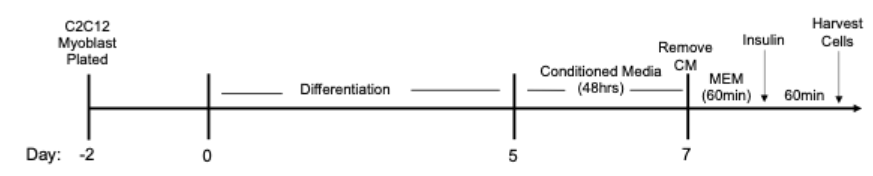

B.

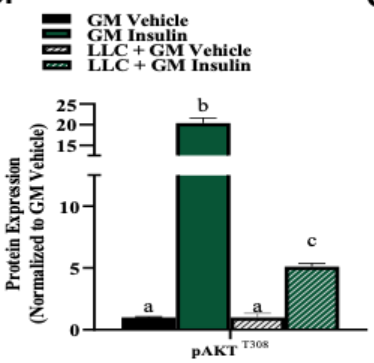

C.

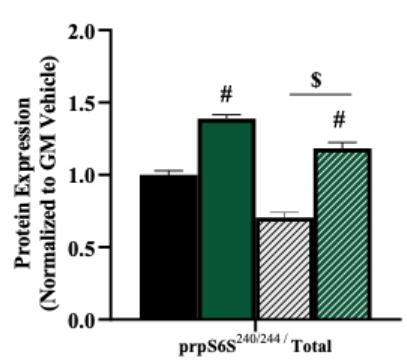

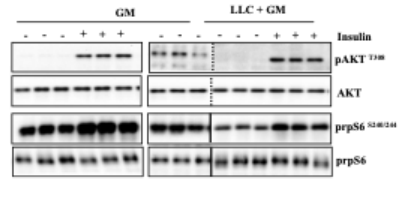

Figure 4-9. LLC conditioned media suppresses the insulin activation of Akt and mTORC1 signaling in C2C12 myotubes

Notes: Data are presented as mean \pm SEM. A. Study Design. B. Phospho-to total ratio of AKT T308. C. Phospho-rpS6 to total ratio. Two-way ANOVAs were used to compare LLC and GM with or without insulin. Different letters mean groups are different. \# Main effect of Insulin, \$ Main effect of LLC. $N=6 / 2$ separate experiments. 
A.

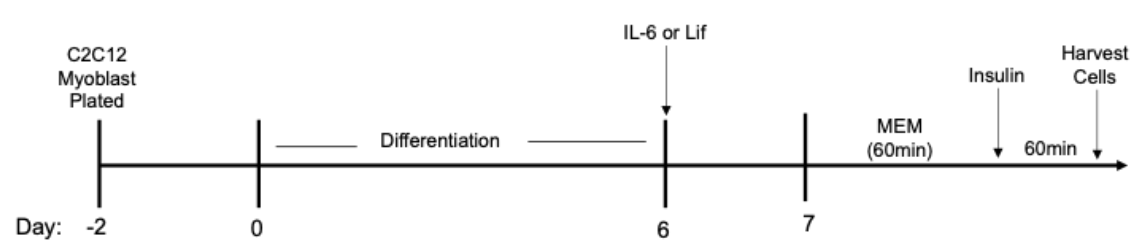

B.

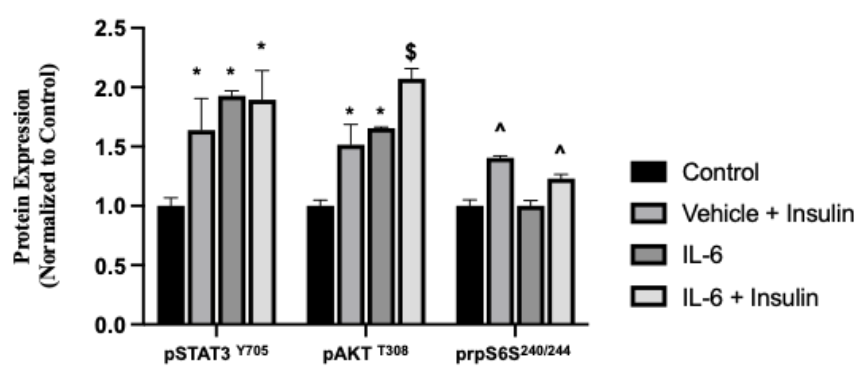

c.

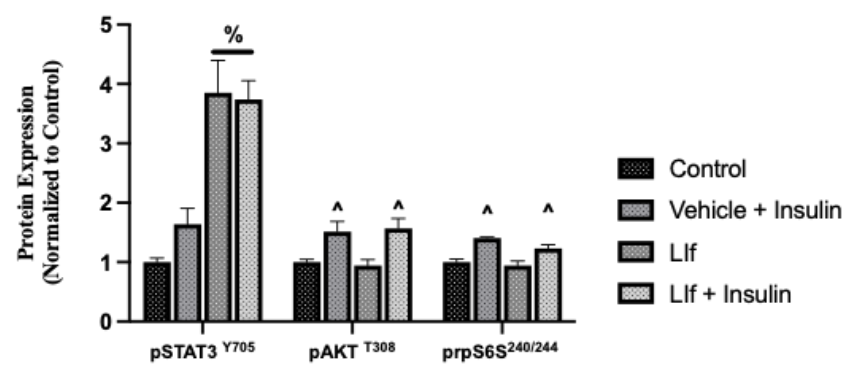

Figure 4-10. IL-6 nor lif were sufficient to suppress Akt/mTORC1 induction by insulin in $\mathrm{C2C12}$ myotubes

Notes: Data are presented as mean \pm SEM. A. Study Design B. Phospho-to total ratio of STAT3, Akt, and rpS6 protein expression in cells treated with IL-6 for 24hrs then given insulin for 60 minutes. C. Phospho-to total ratio of STAT3, Akt, and rpS6 protein expression in cells treated with Lif for $24 \mathrm{hrs}$ then given insulin for 60 minutes. Two-way ANOVAs were used to compare with or without IL-6 or Lif and with or without insulin. *Different from control, \$ Different from all groups, ^ Main effect of Insulin, \% Main effect of Lif. $N=6 / 2$ separate experiments. 


\section{Discussion}

Inflammation has been implicated as a key mediator of cancer-induced cachexia leading to chronic elevation of skeletal muscle STAT3 and MAPK activity (Deans \& Wigmore, 2005). Glycoprotein-130 (gp130) is a regulatory point of interaction between systemic inflammation and JAK/STAT and MAPK muscle signaling (White \& Stephens, 2011). We have previously reported muscle gp130 loss prevented Lewis Lung Carcinoma (LLC) induced muscle wasting and restored STAT3 and MAPK signaling (Puppa, Gao, et al., 2014). In pre-clinical models, our laboratory has demonstrated altered protein turnover through the suppression of anabolic signaling via mTORC1 which coincided with the chronic activation of AMPK (White, Baynes, et al., 2011). Interestingly, cachexia has known disruptions in mitochondrial function prior to muscle wasting (Brown et al., 2017) and theses disruptions to the muscle mitochondria are known to induce AMPK, thereby accelerating muscle wasting (VanderVeen et al., 2017). We report the novel finding that muscle gp130 loss reduced the elevated AMPK but was not sufficient to rescue suppressed protein synthesis in pre-cachectic tumor-bearing mice.. Finally, gp130 loss did not alter the protein synthesis response to feeding in PBS or LLC mice. Given the known regulation of gp130 and associated signaling on muscle wasting, our findings suggest that gp130's regulation of skeletal muscle's response to anabolic stimuli is likely more important in cachectic muscle.

Several studies have implicated the role of gp130 signaling on preventing and/or treating cancer induced cachexia. Briefly, skeletal muscle-specific STAT3 loss prevented LLC induced muscle wasting and grip strength loss, without altering tumor mass (Silva et al., 2015). Interestingly, in a genetic model of cancer induced cachexia (Apc ${ }^{\mathrm{Min} /+}$ mice), IL-6 receptor antibody preserved some body weight and muscle mass loss (White, Baynes, et al., 2011). Furthermore, IL-6 receptor antibody was sufficient in reducing STAT3 and AMPK signaling without rescuing the suppression of mTORC1. Importantly we have shown that gp130 loss prevented muscle wasting in LLC tumor-bearing mice (Puppa, Gao, et al., 2014). Herein, we did not rescue muscle mass loss in gp130 KO LLC mice. There are potentially several reasons for this. First, we report a $50 \%$ reduction in crude muscle gp130 protein expression, crude muscle has several other tissue types, and gp130 is ubiquitously expressed in all tissue types. Furthermore, a sufficient knockout of gp130 is likely because we see downstream associated gp130 signaling reduced.

Secondly, since we did not see differences in tumor development, it's possible that as the tumor develops, the tumor is releasing factors which can contribute to muscle wasting. It is important to mention that we report around 5\% body weight loss, suggesting a precachectic phenotype in our gp130 WT mice, whereas as Puppa et al. show an average body weight loss of 7-10\% (moderate cachexia). Importantly, in Puppa et al, they reported gastrocnemius muscle mass loss of $-17 \%$, whereas as we only report $-9 \%$ further supporting that our LLC tumor-bearing mice were pre-cachectic. Interestingly, this provided an opportunity to understand what gp130 signaling regulates early during cachexia development. Future studies should determine the importance of gp130 signaling in pre-cachectic tumor-bearing mice subjected to high inflammatory environment (such as chemotherapy), as this has significant implications in clinical populations. 
AMPK is chronically elevated during cancer cachexia progression and has shown to regulate mTORC1 signaling and protein synthesis. Additionally, we have examined a role for systemic IL-6 in suppressing protein synthesis during cachexia (White et al., 2013). We have previously shown that IL-6 exposure has the capacity to decrease C $2 \mathrm{C} 12$ myotube diameter and suppress mTORC1 signaling, and when AMPK is blocked the IL6 suppression of protein synthesis is relieved (White et al., 2013). We extend these studies by highlighting that gp130 loss reduced AMPK in tumor-bearing mice, however this inhibition was not sufficient to restore mTORC1 signaling and protein synthesis. These findings agree with cell culture work showing AMPK, but not gp130 inhibition rescued protein synthesis suppression. It is interestingly to speculate why the suppression of AMPK by gp130 loss was not sufficient to improve protein synthesis. We know that elevated AMPK signaling is associated with cachexia progression, therefore it's possible that during early cachexia, the stress induced by fasting does not regulate mTORC1, however this is unlikely given previous data. It is also possible, that in these pre-cachectic mice, AMPK reduction by gp130 loss mice regulates mitochondrial quality control or autophagy prior to rescuing protein synthesis. Lastly, it is very possible that gp130 loss is only minimally targeting AMPK. AMPK can be phosphorylated by LKB1 and $\mathrm{CAMKK} \beta$ (Ca2+/ Calmodulin-dependent protein kinase B) (Kim et al., 2016). We know that LKB1 phosphorylation of AMPK can be improved by binding of AMP to the gamma subunit of AMPK; this alternative is less likely because all mice were fasted therefore, we would suggest that the AMP/ATP ratios might be similar. However, these inquires require further investigation. Alternatively, $\mathrm{CaMKK} \beta$ can phosphorylate AMPK when cellular calcium levels change without changes to AMP/ATP. It is within reason to speculate that the reduction in AMPK by gp130 loss can be attributed to altered calcium within the cell early during cachexia development. However, future work is needed to further understand the implications of lowered AMPK without improved protein synthesis in the absence of muscle gp130 in tumor-bearing mice.

Cachexia has known disruptions in mitochondrial function prior to muscle wasting (Brown et al., 2017) and theses disruptions to muscle mitochondria are known to induce AMPK, thereby accelerating muscle wasting (VanderVeen et al., 2017). We extend previous studies by highlighting that gp130 loss in pre-cachectic tumor-bearing mice did not improve the PGC1 $\alpha$ suppression. Given that muscle gp130 can regulate mitochondrial quality control (Hardee, Counts, et al., 2018), it is within reason to speculate that in these pre-cachectic mice, gp130's regulation of mitochondrial quality control is not exerted till later stages cachexia when the inflammatory environment is increased. If is this true, then early disruptions to mitochondria are occurring independent of gp130. This is likely further supported by the relatively low expression of circulating IL-6, whereas previous studies highlighting beneficial effect of gp130 loss reported a 2fold greater induction of IL-6. It is interesting to speculate if the early disruptions in mitochondrial biogenesis are due to impaired lipid oxidation (Counts et al., 2021). Additionally, given the known regulation of increased physical activity on improving mitochondrial biogenesis, and pre-cachectic patients and tumor-bearing mice exhibit reduced physical activity, it is possible that early disruptions to mitochondria are attributed to physical inactivity. However, future studies are needed to delineate the 
effects of physical inactivity and gp130's regulation of mitochondria in order to further understand the mitochondrial regulation during the progression of cachexia.

While a majority of our understanding of protein synthesis during cachexia is examined in murine models, protein synthesis rates are reduced in cachectic patients (Dworzak et al., 1998). We have routinely shown suppressed protein synthesis during the progression of cachexia in $A p c^{\mathrm{Min} /+}$ mice, and this suppression corresponded to a reduction in muscle protein mTORC1 and IGF1 (White, Baynes, et al., 2011). The early suppression of muscle protein synthesis during cachexia did not correspond to an initial change in AMPK, since AMPK activity does not increase till late-stage cachexia (White, Baynes, et al., 2011). However, we have recently shown that tumor-bearing mice have an accelerated fasting induction of AMPK signaling early during cachexia progression (Fix et al., 2021). Herein we have extended previous studies by showing mTORC1 signaling is suppressed in the fasting condition but is not regulated by gp130. Furthermore, gp130 loss did not alter the protein synthesis induction by feeding. Since we know that elevated gp130 associated signaling can suppress mTORC1 signaling and protein synthesis, it is interesting to speculate that under basal conditions gp130 loss is not required for the feeding regulation $\mathrm{mTORC} 1$ and protein synthesis. Importantly, we show that in precachectic mice the anabolic suppression of $\mathrm{mTORC} 1$ and protein synthesis by feeding is independent of gp130. Since we know STAT3 signaling is associated to cachexia progression, it is interesting to speculate that during the transition to a more cachectic phenotype, gp130 can regulate the muscle's feeding response. While previous studies have shown little regulation of mTORC1 by gp130 loss in cachectic muscle, it does not discount the potential inhibitory role of gp130 following an anabolic stimulus. Therefore, future studies should determine if gp130 regulates the feeding induction of mTORC1 signaling and protein synthesis during severe cachexia.

No study is without limitations. A limitation of this study is that we examined males, not females. Sex as a biological variable, especially during cancer cachexia has only minimally been investigated. While this warrants further examination there are several considerations that need to be addressed. First, we used tamoxifen-inducible muscle-specific knockout mice, tamoxifen binds to the estrogen receptors throughout the body thereby inhibiting the receptors effects on tissues. It has yet to be considered if the sudden loss of estrogen associated signaling impact on skeletal muscle, in an environment with high estrogen levels, females. Next, indices of cachexia are less common in female mice compared to male mice, which has been linked to hypogonadism. Additionally, there is a possibility that the 1-hr time difference between fed and fast conditions could intrinsically be different. However, there is sufficient circadian rhythm data to identify that this 1-hr difference between sacrifice times will not elicit an intrinsic difference in muscle signaling. Therefore, it is very unlikely that the 1$\mathrm{hr}$ time difference is going to affect skeletal muscle signaling. The LLC model is not an orthotopic model of lung cancer. There are potential alternative approaches to examining lung cancer in pre-clinical models however, orthotopic models of lung cancer are commonly completed in Balb/c mice or immunodeficient mice, which does not allow for examination of muscle-specific inducible models or for the normal immune response. Furthermore, cachexia progression in female mice is initially IL-6/gp130/STAT3 
independent, therefore it does not appear necessary at this stage to examine the role of gp130 during cachexia progression in female mice. Therefore, our study only used male mice due to several of our methodologies can be differentially regulated or not effected in the female tumor-bearing mouse. Lastly, some transgenic models of cancer cachexia utilize tamoxifen administration, a tumor inducer, and would be a significant confounder when using to elicit a muscle-specific knockout (Kellar et al., 2015; Safari \& Meuwissen, 2015). Importantly, the LLC model is commonly used due to its tumorigenic properties, responsiveness to chemotherapy, and ability to metastasize to the lungs and liver (Kellar et al., 2015) thus allowing us to use a well characterized model to examine cancer cachexia progression.

\section{Conclusion}

In conclusion, gp130 loss did not prevent muscle wasting in pre-cachectic tumorbearing mice. Interestingly, gp130 loss was sufficient to reduce elevated AMPK, without improving downstream mTORC1 signaling and protein synthesis in pre-cachectic mice. Importantly, gp130 loss did not impair the protein synthesis response to feeding in tumorbearing mice. Taken together, we provide the novel understanding that gp130's regulation of skeletal muscle's response to anabolic stimuli is likely more important during later stages of cachexia. Studies are needed to delineate the early effects of gp130 on skeletal muscle homeostasis in order to provide therapeutic targets to prevent cachexia. Future studies should examine if STAT3 inhibition in cachectic muscle can improve the fasting regulation of AMPK and protein synthesis. Additionally, future studies are warranted to examine why gp130 loss lowers AMPK in pre-cachectic skeletal muscle without improving the suppressed protein synthesis. 


\section{CHAPTER 5. EXAMINATION OF WHETHER AN ACUTE BOUT OF MUSCLE CONTRACTION CAN IMPROVE MUSCLE PROTEIN TURNOVER RESPONSE TO FEEDING DURING THE PROGRESSION OF CACHEXIA}

\section{Introduction}

Cancer cachexia exhibits reduced volitional activity, whole body weakness, and fatigue in cancer patients and in pre-clinical models (Baltgalvis et al., 2010; Murphy et al., 2012; Toth et al., 2016). Furthermore, contractile decrements have also been reported (Christensen et al., 2014; Roberts, Frye, et al., 2013), and are highly important given that force production and fatigue are directly related to life quality in disease (al-Majid \& McCarthy, 2001; Barreiro \& Gea, 2015; Siegel, 1989). We and others have recently shown that cachectic skeletal muscle develops a slow-fatigable contractile phenotype during cancer cachexia progression (Murphy et al., 2012; Roberts, Frye, et al., 2013; VanderVeen et al., 2018). Furthermore, the regulation of muscle's contractile properties are strongly related to the muscle's inflammatory signaling highlighting the regulation of muscle contraction by inflammation during disease (VanderVeen et al., 2019). While fatigue and weakness are the most commonly reported consequences in cancer patients, we have only scratched the surface on trying to understand how this may occur (Chang et al., 2000). Interestingly, recent work has highlighted exercise's therapeutic role in improving muscle fatigability in patients and pre-clinical models (Kessels et al., 2018; Vanderveen et al., 2020). Several indices of cachexia progression are attenuated in rodents given access to a running wheel by attenuating muscle mass loss and improving myofiber cross-sectional area (Coletti et al., 2016; Pigna et al., 2016). These studies highlight the improved ex-vivo force production and reduced inflammatory environment likely attribute to the prevention of cachexia. In addition, treadmill training has also been shown to offset indices of cachexia and was sufficient to decrease total polyp number (Mehl et al., 2005), likely through reducing immune infiltration, apoptosis, and betacatenin signaling (Baltgalvis et al., 2010). Furthermore, exercise training prior to and during IL-6 overexpression prevented muscle mass loss, improved systemic glucose, and restored mTORC1 signaling (Lombardi et al., 2012; White et al., 2013). Exercise has recognized benefits for the prevention of some cancers and electrical muscle stimulation has beneficial effects in critically ill patients (Gerovasili et al., 2009). However, the mechanisms of how exercise can be a therapeutic have to yet to be fully understood.

Exercise training is a known inducer of anabolic signaling through mTORC1 (Burd et al., 2009) and reduces chronic systemic inflammation (Beavers et al., 2010). An acute bout of skeletal muscle contraction is sufficient to induce glucose uptake, fatty acid oxidation, mitochondrial biogenesis, and protein synthesis (Egan \& Zierath, 2013). Whereas repeated exercise bouts can induce functional adaptations in contractile proteins, mitochondrial function, and metabolic regulation (Egan \& Zierath, 2013). After an acute bout of exercise PGC-1 $\alpha$ is rapidly up-regulated leading to a subsequent increase in mitochondrial associated gene transcription and mitochondrial biogenesis (Baar et al., 2002; Pilegaard et al., 2003). Up-regulation of these genes persists for up to 4 hours before returning to baseline levels. Exercise has been hypothesized to be a potential 
therapeutic option for cancer induced cachexia (Hardee et al., 2019). We have recently shown that repeated eccentric contractions are sufficient to induce anabolic signaling in the cachectic environment, coinciding with suppressed inflammatory signaling in glycolytic muscle (Hardee et al., 2020). Additionally, we highlight that repeated contractions improved muscle oxidative capacity further supporting that cachectic muscle has the plasticity to respond to contraction stimulus. While high frequency eccentric contractions show promising results in preclinical models, clinical populations hesitate to employ such a potent stimulus therefore rehabilitation settings utilize a low-to-moderate frequency of contraction/load (Hody et al., 2019). We have previously shown that an acute bout of low-frequency electrical stimulation (LFES) induced downstream mTORC1 phosphorylation and oxidative capacity, however this response was suppressed in cachectic muscle (Puppa, Murphy, et al., 2014). While we have made advances in our understanding of cachectic muscle's response to acute contraction, significant barriers still exist in understanding if the suppressed contraction occurs early during cachexia progression.

Skeletal muscle mitochondrial function plays a pivotal role in muscle glucose uptake, and impairments in oxidative capacity have been associated with insulin resistance (Lanza \& Nair, 2009). AMPK is a critical regulator for the exercise response in skeletal muscle. An acute exercise bout increases protein levels of AMPK, despite this exercise training induced an increase in muscle protein synthesis even in the cachectic environment. Exercise is also able to attenuate the chronic activation of AMPK that is seen with severe muscle wasting (Lombardi et al., 2012). The improvements in the regulation of AMPK activity may be due to the improvements in glucose uptake which could alleviate the energy stress that is placed on the muscle due to the cancer environment and metabolic inflexibility. More research needs to be done to examine the effects of exercise on muscle after the initiation of cachexia and if exercise can rescue mitochondrial dynamics and function. However, most exercise interventions are used as a prevention. Additionally, several animal models use a volitional modality of exercise which provides variability in treadmill and wheel exercise; electrical stimulation is another exercise modality often used to elicit skeletal muscle contractions. Skeletal muscle's metabolic and growth response to contraction have been examined using electrical muscle stimulation (Baar \& Esser, 1999; Nader \& Esser, 2001; Puppa, Murphy, et al., 2014; Witkowski et al., 2010). In pre-clinical models, electrical stimulation is performed in an unconscious animal, which electrodes are used to stimulate a nerve at a specific frequency. Several studies have examined skeletal muscle anabolic signaling following high-frequency electrical muscle stimulation (HFES) (Baar \& Esser, 1999; Hardee et al., 2016; Sato et al., 2019), which induced mTORC1 associated signaling and protein synthesis. In contrast, low-frequency electrical muscle stimulation (LFES) has been used to examine the acute and training adaptations to endurance like muscle contractions (Nader \& Esser, 2001; Puppa, Murphy, et al., 2014). An acute exercise bout of low frequency stimulation can induce mTORC1 signaling (Tsutaki et al., 2013). In human populations, a single bout of LFES improved glucose uptake (Hamada et al., 2003) and 4wks of LFES induced markers of glucose metabolism and oxidative capacity (Theriault et al., 1994). Furthermore, 4wks of LFES improved fatigue resistance following spinal cord injury (Harridge et al., 2002). Taken together, LFES has therapeutic 
potential, however more work is needed in order to under the molecular mechanisms of exercise in chronic disease.

The anabolic response of resistance exercise in combination with nutritional stimulus has been highly sought after in all populations (Barclay et al., 2019; Breen \& Phillips, 2013; Moore, 2019; Symons et al., 2011; Trommelen et al., 2019); specifically, in the elderly or during disease in hopes to offset anabolic resistance. Several studies have investigated skeletal muscle's response to contraction and/or mechanical signaling (Hornberger, 2011), however very little work has been done to examine the combined effects of contraction and feeding on skeletal anabolic signaling (Gordon et al., 2014; Nakayama et al., 2019) specifically during disease (Hardee, Counts, et al., 2018; Puppa, Murphy, et al., 2014; Sato et al., 2019) and disuse (Roberson et al., 2020; Shimkus et al., 2018). Interestingly, there is promising work that the combined effects of exercise and nutrient supplementation can increase muscle protein synthesis to a greater extent than feeding alone in cancer patients (Hanson et al., 2017). Given that we know cachectic muscle has a suppressed anabolic response to muscle contractions, gaps remain in our understanding if tumor-bearing mice have to capacity to synergistically induce protein synthesis in response to contraction and feeding. Therefore, the purpose of aim 3 was to determine if an acute bout of muscle contraction could improve the muscle protein synthesis response to feeding during the progression of cachexia. We hypothesized that an acute bout of muscle contraction could improve muscle protein turnover response to feeding, and the response would be greater during pre-cachexia compared to refractory cachexia.

\section{Methods}

\section{Animals}

Male C57BL/6J (B6; N=36) mice were bred at the University of Tennessee Health Science Center Animal Resource Facility. B6 mice were initially purchased from Jackson Labs (Bar Harbor, ME, USA). Mice were kept on a 12:12h light/dark cycle beginning at 6:00AM and were given rodent chow ad libitum (Harlan Teklad Rodent Diet, \#8604, Harlan, Indianapolis, IN, USA). All mice were fasted for $12 \mathrm{hrs}$ during the dark cycle. At the start of the light cycle, mice were either given ad libitum access to a food pellet (fed) or sacrificed at the end of fast (fast). Mice then underwent low frequency electrical stimulation and sacrificed $3 \mathrm{hrs}$ post.

\section{Lewis lung carcinoma cell inoculation}

Between 11-12 weeks of age, B6 mice were injected with either phosphate buffered saline (PBS) or $1 \times 10^{6}$ Lewis lung carcinoma (LLC) cells subcutaneously in the right flank under anesthesia for less than 3 minutes (Puppa, Gao, et al., 2014). A total of 22 mice were injected with LLC cells and 19 mice were injected with PBS. Seventyseven percent of the LLC injected mice reached the studies endpoint. A mouse reached 
the studies endpoint if the following criteria were met (1) mouse reached 30 days post tumor inoculation, (2) $>20 \%$ body weight loss from day 10 after day 25 , or (3) had a tumor $>3 \mathrm{~cm}$ in width or length after day 25 . Once an endpoint was achieved, the mouse was prepared for tissue collection and euthanized within $24 \mathrm{hrs}$. At day 20, 2 mice had ulcerated tumors and was not included in the analysis and 1 mouse died unexpectedly at day 21. At day 24, 2 mice had ulcerated tumors and were not included in the analysis. To be included in the study, mice needed to achieve at least 25 days post tumor inoculation. Therefore, a total of 17 male LLC injected mice were used in these experiments. Tumor volume and body weight were measured every 5 days to calculate tumor growth. Tumor volume was calculated by the same investigator and caliper using the following equation: $1 / 2$ (width ${ }^{2}$ xlength) (Jensen et al., 2008).

\section{Low frequency electrical stimulation}

LFES was conducted as described by (Nader \& Esser, 2001) with slight modifications. Briefly, all animals were fasted during the dark cycle for $12 \mathrm{hr}$ before stimulation. At the end of the $12 \mathrm{hrs}$, mice were either given access to a food pellet for $1 \mathrm{hr}$ or maintained in the fast condition, mice were then subjected to an acute bout of LFES. Animals were anesthetized with isoflurane in a chamber at 3-4\% and remained anesthetized for the procedure via a nose cone that was connected to the isofluraneoxygen. Animals were placed on a heat pad, and the left hindlimb was shaved free of hair and cleaned with alcohol. Electrodes were placed on both sides of the tibial nerve and stimulated via subcutaneous needle. Proper electrode position was confirmed by observing plantar flexion at the ankle joint. This protocol elicited an overall effect of plantar flexion, resulting in tapping of the foot. The voltage was applied by Aurora Scientific (Aurora Scientific, Ontario, Canada). Stimulation was delivered at a frequency of $10 \mathrm{~Hz}, 5 \mathrm{~V}, 10$-ms duration, 90-ms delay, for a total time of $30 \mathrm{~min}$. Mice were then sacrificed $3 \mathrm{hrs}$ after the stimulation.

\section{Blood glucose and plasma insulin}

Immediately prior to ad libitum access to a food pellet, mice had their tail snipped. The initial blood was wiped with a Kim wipe and blood glucose was determined using a standard and readily available glucometer (Contour Next, Parsippany, NJ). Blood was collected with a heparinized capillary tube, placed on ice, and centrifuged $(10,000 \mathrm{x}$ $\mathrm{g}$ for $10 \mathrm{~min}$ at $4^{\circ} \mathrm{C}$ ). The supernatant was removed, and plasma insulin concentrations were determined using a two-site enzyme immunoassay assay kit according to the manufacturer's instructions (Catalog \#10-1247, Mercodia, Uppsala, Sweden). Blood glucose and plasma insulin measurements were taken again 1-hr after access to the food pellet and prior to anesthesia at sacrifice. 


\section{Western blot}

Western blot analysis was performed as previously described (Hardee et al., 2014) and extensive methodology can be found in extended methods section. Briefly, frozen red and white gastrocnemius muscle was homogenized in lysis buffer and protein concentration was determined by the Bradford method. Crude gastrocnemius muscle homogenates were fractionated on $8-12 \%$ SDS-polyacrylamide gels and transferred to PVDF membranes. Membranes were stained with Ponceau red to verify equal loading and transfer. Membranes were then blocked at room temperature for $1 \mathrm{hr}$. in 5\% non-fat milk or 5\% BSA-Tris-buffered saline with 0.1\% Tween-20 (TBST). Commercially available phosphorylated and total protein primary antibodies for rpS6, AKT, AMPK, STAT3, and DRP1 were raised in rabbit. RpS6, AKT, AMPK, STAT3, and DRP1 phosphorylation antibodies were expressed relative to total protein on the same gel and quantified as phosphorylation to total ratio. Commercially available total protein primary antibodies for PGC1 $\alpha$, and FIS1 were raised in rabbit. Puromycin incorporation was purchased from Millipore and raised in mouse. Briefly, primary antibodies were incubated overnight in 5\% TBST milk or 5\% TBST BSA. Membranes were then incubated in 5\% milk-TBST containing anti-rabbit or anti-mouse IgG horseradishperoxidase conjugated secondary antibody purchased from cell signaling for $1 \mathrm{~h}$ at room temperature. Enhanced chemiluminescence (ECL) (GE Healthcare Life Sciences, Piscataway, NJ) was used to visualize the antibody-antigen interactions. Immunoblot images were collected using a digital imager (Invitrogen iBright, Waltham, MA) and quantified by densitometry using imaging software (Image J; NIH). Please see end of document for details of catalogue numbers, companies, dilutions, incubation durations, and secondary substrate (Figure A-1).

\section{Statistical analysis}

Pre-planned unpaired T-Test were used to compare the feeding and contraction response in PBS and LLC mice. Two-way (Tumor x LFES) (Feeding x LFES) ANOVAs were used, and Tukey's post-hoc analysis was used when appropriate. Pearson spearman correlations were used to associate skeletal muscle signaling with indices of cachexia. Prism 8 (GraphPad) was used for statistical analysis. All results are reported as means \pm standard error of mean (SEM). Statistical analysis was performed using GraphPad (Prism 8 for Mac OS X, La Jolla, Ca). Level of significance for all measures was set at $\mathrm{p}$ $\leq 0.05$.

\section{Results}

Between 11-12 weeks of age C57BL/6J (B6) mice were injected with phosphate buffered saline (PBS) or Lewis lung carcinoma (LLC) cells and were then sacrificed 2530 post injection. There were no differences in age or study duration between PBS $(\mathrm{N}=19)$ and LLC $(\mathrm{N}=17)$ mice. The study was initiated at approximately 12 weeks of age and completed at approximately 15 to 16 weeks of age. PBS and LLC mice bodyweights 
were similar before tumor inoculation and 10 days post tumor inoculation (Table 5-1). While there were no differences in absolute body weight at the end of the study, after accounting for tumor mass, LLC mice had decreased body weight compared to PBS mice. LLC mice had a greater body weight change from day 10 when accounting for tumor mass $(-4.0 \%)$ compared to PBS mice $(2.9 \%)$. Body weight change from day 10 was chosen because the tumor is initially palpable but prior to the rapid tumor growth phase (days 15-25). LLC mice had decreased total hindlimb muscle mass (-18\%) and eWAT mass $(-25 \%)$ compared to PBS mice. LLC mice had increased spleen mass and plasma IL-6 compared to PBS mice. Tumor volume and tumor mass were used to validate tumor development. Tumor volume was measured every 5 days throughout the study and was measurable 10 days post tumor inoculation. Utilizing the LLC model's rapid tumor growth phase, we calculated the slope of tumor growth from days 15-25. Tumor growth rate ranged from 0.029 to $0.645 \mathrm{~cm}^{3} / 5$ days. Tumor mass ranged from 0.993 to 4.84 grams (Table 5-1).

To examine the effect of feeding on systemic metabolism and muscle signaling, we first needed to examine if there were differences in animal characteristics between the fast and fed condition in both PBS and LLC treated mice (Table 5-2). PBS and LLC mice consumed similar amounts of food during the 1-hr ad libitum feeding ( $\mathrm{p}=0.959)$. There was an interaction for blood glucose post the 1-hr ad libitum feeding to be increased compared to fast ( $\mathrm{p}=0.027)$. There were no differences between PBS and LLC fast mice. PBS fed mice increased blood glucose compared to PBS fast. Additionally, LLC fed mice increased blood glucose compared to LLC fast, and was greater than PBS fed. There was a main effect for fed mice to have increased plasma insulin post pellet independent of PBS or LLC $(\mathrm{p}<0.001)$. At sacrifice, there was a main effect for fed groups, independent of condition, to increase blood glucose $(p<0.001)$. There was a main effect for LLC to have increased blood glucose compared to PBS. At sacrifice, plasma insulin was increased in fed compared to fast mice, independent of condition $(p=0.004)$. At sacrifice, there was a main effect of liver mass to be increased compared to PBS independent of feeding status $(\mathrm{p}=0.003)$. Additionally, stomach mass was increased in fed mice, independent of PBS or LLC $(\mathrm{p}<0.001)$. As anticipated, there were no differences in empty stomach mass in PBS or LLC mice in either the fed of fast condition. There was a main effect of stomach content to be greater in fed mice, compared to fast mice for either PBS or LLC $(\mathrm{p}<0.001)$. Taken together, these results validate the whole-body response to feeding in both PBS and LLC mice.

Next, we sought to first validate the acute contraction response by LFES in PBS male mice (Figure 5-1). We report that LFES induced rpS6 9.5-fold, puromycin incorporation 4.7-fold, and PGC1 $\alpha$ 0.7-fold in the stim leg compared to the non-stim leg. In LLC mice, we report that LFES induced rpS6 1.7-fold, puromycin incorporation 0.9fold, but was not sufficient to increase PGC1 $\alpha$ in the stim leg compared to the non-stim leg (Figure 5-1). While LLC mice were able to induce mTORC1 signaling and protein synthesis, the overall response was lower in the LLC mice compared to PBS (rpS6 decreased 2-fold and protein synthesis decreased 3-fold in LLC compared to PBS). Furthermore, the induction of mTORC1 and protein synthesis was not associated with indices of cachexia. Taken together, we highlight that LLC tumor-bearing mice can 
Table 5-1. Animal characteristics of PBS and LLC mice in the fast and fed condition 3hrs post LFES

\begin{tabular}{|c|c|c|c|c|}
\hline \multirow[b]{2}{*}{ Characteristics } & \multicolumn{2}{|c|}{ PBS } & \multicolumn{2}{|c|}{ LLC } \\
\hline & $\begin{array}{c}\text { Fast } \\
\mathrm{N}=10\end{array}$ & $\begin{array}{l}\text { Fed } \\
N=9\end{array}$ & $\begin{array}{l}\text { Fast } \\
\mathrm{N}=8\end{array}$ & $\begin{array}{l}\text { Fed } \\
N=9\end{array}$ \\
\hline BW Pre-treatment (g) & $25.7(0.4)$ & $24.5(0.8)$ & $24.3(0.8)$ & $24.3(0.7)$ \\
\hline BW d10 (g) & $26.6(0.5) \mathrm{a}$ & $25.8(0.7) \mathrm{a}$ & $25.6(0.6)$ & $25.4(0.7) \mathrm{a}$ \\
\hline BW End of Study (g) & $27.2(0.6)$ & $26.8(0.6)$ & $27.8(1.0)$ & $27.2(0.7)$ \\
\hline BW End of Study & $27.2(0.6) \mathrm{a}$ & $26.8(0.6) \mathrm{a}, \mathrm{b}$ & $24.8(0.8) \mathrm{a} \#$ & $24.2(0.8) \mathrm{b} \#$ \\
\hline Tumor Mass (g) & - & - & $3.0(0.5)$ & $3.1(0.4)$ \\
\hline $\mathrm{BW} \Delta$ during treatment $(\%)$ & $5.7(1.0)$ & $9.5(1.1)$ & $0.9(1.8) \#$ & $-0.7(1.6) \#$ \\
\hline $\mathrm{BW} \Delta \mathrm{d} 10(\%)$ & $2.0(0.8)$ & $3.8(0.8)$ & $-3.2(1.5) \#$ & $-4.8(1.3) \#$ \\
\hline Tumor Volume d15 $\left(\mathrm{cm}^{3}\right)$ & - & - & $0.332(0.120) a, b$ & $0.311(0.100) a, b$ \\
\hline Tumor Volume d20 $\left(\mathrm{cm}^{3}\right)$ & - & - & $1.042(0.365) \mathrm{a}$ & $1.339(0.355) \mathrm{b}$ \\
\hline Tumor Volume d25 $\left(\mathrm{cm}^{3}\right)$ & - & - & $3.103(0.772) b$ & $2.584(0.529) \mathrm{a}$ \\
\hline Tumor Slope Rate d15-25 & - & - & $0.277(0.071)$ & $0.231(0.046)$ \\
\hline HMM (mg) & $194(4)$ & $192(5)$ & 179 (5)\# & 176 (6)\# \\
\hline eWAT (mg) & $306(26)$ & $241(14)$ & $208(7) \#$ & $202(23) \#$ \\
\hline Testes (mg) & $204(6)$ & $198(4)$ & $187(7)$ & $194(10)$ \\
\hline Spleen (mg) & $63(3)$ & $59(2)$ & $243(32) \#$ & $246(40) \#$ \\
\hline Tibia Length $(\mathrm{mm})$ & $16.7(0.1)$ & $16.6(0.2)$ & $16.8(0.1)$ & $16.6(0.1)$ \\
\hline
\end{tabular}

Notes: Data are presented as mean \pm SEM. Abbreviations: BW: body weight, mg: milligrams, eWAT: epididymal white adipose tissue, PBS: Phosphate buffered saline, LLC: Lewis lung carcinoma, mm: millimeter, g: grams, ME: Main Effect, HMM: Hindlimb muscle mass. One-way repeated measures ANOVA was used to compare within LLC fast and fed mice body weight over the course of the study (BW End of study is not included in analysis/ BW end of study accounting for tumor mass is included) ${ }^{a}$ Different from Pre within treatment group, ${ }^{\mathrm{b}}$ Different from BW d10 within treatment group. One-way repeated measures ANOVA was used to compare tumor volume over the course of the study. ${ }^{\text {a }}$ Different from d15 within treatment group, ${ }^{b}$ Different from d20 within treatment group. Statistically significant $p<0.05$. Two-way ANOVAs were used to compare PBS and LLC in the Fast and Fed condition. \# Main Effect of LLC. Unpaired Ttest were used to compare tumor growth and mass in the fed and fast condition of LLC mice. * Different from LLC fast. 
Table 5-2. Whole-body response to feeding in PBS and LLC male mice

\begin{tabular}{|c|c|c|c|c|}
\hline \multirow[b]{2}{*}{ Characteristics } & \multicolumn{2}{|c|}{ PBS } & \multicolumn{2}{|c|}{ LLC } \\
\hline & $\begin{array}{c}\text { Fast } \\
\mathrm{N}=10\end{array}$ & $\begin{array}{l}\text { Fed } \\
\mathrm{N}=9\end{array}$ & $\begin{array}{l}\text { Fast } \\
\mathrm{N}=8\end{array}$ & $\begin{array}{l}\text { Fed } \\
\mathrm{N}=9\end{array}$ \\
\hline Pellet Consumption (g) & - & $0.78(0.11)$ & - & $0.79(0.06)$ \\
\hline Blood Glucose Post-Feeding (mg/dl) & $92(3) \mathrm{a}$ & $190(6) b$ & $97(9) \mathrm{a}$ & $228(7) \mathrm{a}$ \\
\hline Blood Glucose @ Sac. (mg/dl) & $12(8)$ & $159(6) \#$ & $133(15) \$$ & $182(4) \# \$$ \\
\hline Plasma Insulin post-feeding ( $\mathrm{ng} / \mathrm{mL})$ & $0.241(0.037)$ & $2.604(0.394) \#$ & $0.243(0.039)$ & $1.961(0.217) \#$ \\
\hline Plasma Insulin@Sac. (ng/mL) & $0.207(0.003)$ & $0.452(0.090) \#$ & $0.295(0.072)$ & $0.531(0.113) \#$ \\
\hline Liver Mass (g) & $1.2(0.0)$ & $1.2(0.0)$ & $1.4(0.1) \$$ & $1.3(0.1) \$$ \\
\hline Stomach Mass (mg) & $381(30)$ & $763(100) \#$ & $424(41)$ & $586(48) \#$ \\
\hline Empty Stomach (mg) & $159(7)$ & $170(10)$ & $161(8)$ & $143(4)$ \\
\hline Stomach Content (mg) & $222(28)$ & $593(101) \#$ & $263(37)$ & $443(48) \#$ \\
\hline
\end{tabular}

Notes: Data are presented as mean \pm SEM. Abbreviations: mg: milligrams, PBS: phosphate buffered saline, LLC: Lewis lung carcinoma, g: grams, mg/dl: milligrams per deciliter, ng/mL: nanograms per milliliter, Sac.: At Sacrifice, and ME: Main Effect. Unpaired T-test was used to compare PBS and LLC fed pellet consumption. Two-way ANOVAs were used to compare PBS and LLC in the Fast and Fed condition. \#ME of Fed. \$ME of Tumor. If an interaction was present; different letters were used to differentiate differences between groups. Statistically significant $p<0.05$. 


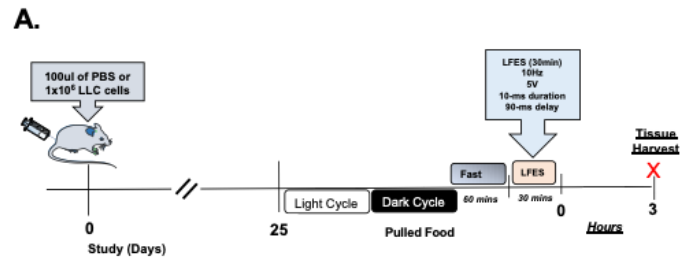

B.

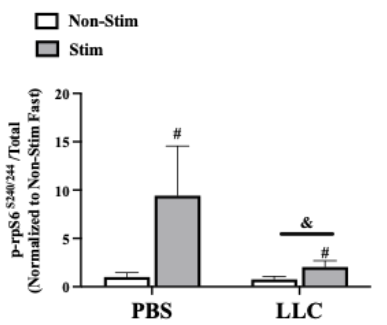

E.

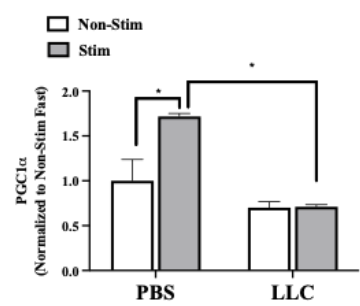

c.

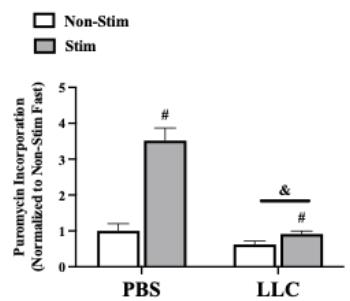

F.

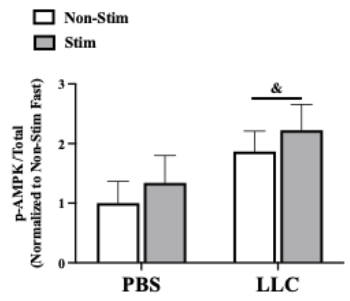

D.

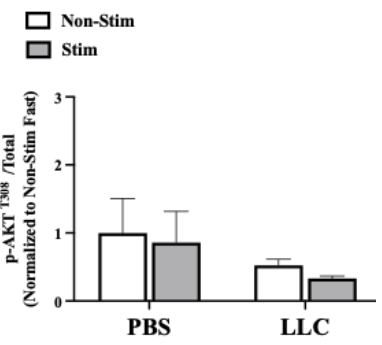

G.

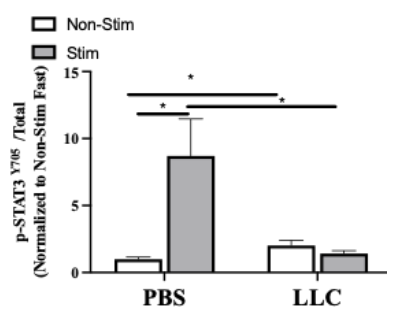

Figure 5-1. LLC tumor-bearing mice exhibit a blunted protein synthesis response to LFES

Notes: Data are presented as mean \pm SEM. A. Study Design. B. Phospho-rps6 to total ratio in PBS and LLC fasted mice 3 hrs after LFES. Non-Stim leg was used as control. C.Puromycin Incorporation in PBS and LLC fasted mice 3hrs after LFES. Non-Stim leg was used as control. D. Phospho-AKT to total ratio in PBS and LLC fasted mice 3 hrs after LFES. E. PGC1 $\alpha$ protein expression in PBS and LLC fasted mice 3 hrs after LFES. F. Phospho-AMPK to total ratio in PBS and LLC fasted mice 3 hrs after LFES. G.Phospho-STAT3 to total ratio in PBS and LLC fasted mice $3 \mathrm{hrs}$ after LFES. Non-Stim leg was used as control. Protein expression is completed in white gastrocnemius muscle. Two-way repeated measures ANOVA were used to compare within animal of the nonstim to stim leg in PBS and LLC mice. Abbreviations: PBS: phosphate buffered saline, LLC: Lewis lung carcinoma. \# Main effect of Stim, \& Main effect of LLC. * Groups are different 
respond to LFES; however, the overall protein synthesis response is suppressed in LLC mice. The added protein synthesis response to feeding and contraction is blunted in precachectic tumor-bearing mice. Furthermore, we show that LFES was not sufficient to increase PGC $1 \alpha$ in pre-cachectic tumor-bearing mice. Taken together, LLC tumorbearing mice exhibit suppressed anabolic signaling and do not synergistically respond to feeding and contraction to examine regulators of muscle protein turnover, we examined AMPK and STAT3 signaling, which are known to be regulated by contraction and cancer cachexia progression. We report that LLC tumor-bearing mice have increased AMPK (1.5-fold), and LFES did not alter this response (Figure 5-1). Interestingly, STAT3 was induced by LFES in PBS mice, but was not induced in LLC mice (Figure 5-1). In the non-stim leg STAT3 was elevated in LLC mice compared to PBS. Taken together, we suggest that the suppressed mTORC1 signaling and protein synthesis in LLC mice, might be attributed to disrupted AMPK and STAT3 signaling following acute contractions

Next, we sought to examine if this blunted anabolic response to contraction can be improved with the combination of feeding, therefore answering the question if LLC tumor-bearing mice exhibit a synergist anabolic response to feeding and contraction. First, we established the synergistic effect of feeding and contraction in non-tumorbearing mice (Figure 5-2). To validate the feeding induction, we report that AKT was induced in fed mice independent of contraction (Figure 5-2). Furthermore, we report a main effect for LFES and a main effect for feeding to increase rpS6 and puromycin incorporation (Figure 5-2). Our results validate the synergistic effect of feeding and LFES in non-tumor-bearing mice. Next we determined if tumor-bearing mice exerted a synergist effect of feeding and contraction. We report feeding increased AKT in LLC mice, which was not altered by LFES (Figure 5-3) nor was it associated with indices of cachexia. LLC tumor-bearing mice increased prpS6 and puromycin incorporation in the stim leg, which was not further induced by feeding (Figure 5-3). Taken together, our results suggest that LLC tumor-bearing mice do not have the capacity to elicit a synergistic anabolic response to feeding and contraction. Lastly, we sought to examine if feeding and contraction can regulate AMPK or STAT3 protein expression. LFES nor feeding altered AMPK signaling in PBS mice (Figure 5-4). Interestingly, feeding induced AMPK in LLC mice (Figure 5-5). Feeding suppressed the STAT3 induction of LFES in PBS (Figure 5-5), and STAT3 was not altered in LLC tumor-bearing mice (Figure 5-5). Taken together, these results suggest that feeding and contraction can both regulate STAT3 protein expression, and LLC disrupts this response.

\section{Discussion}

Cancer cachexia exhibits reduced volitional activity, whole body weakness, and fatigue in cancer patients and in pre-clinical models. Exercise has been hypothesized to be a potential therapeutic option for cancer induced cachexia. We have previously shown an acute bout of low-frequency electrical stimulation (LFES) induced downstream mTORC1 phosphorylation and oxidative capacity, however this response was suppressed in cachectic muscle. There is promising work that the combined effects of exercise and nutrient supplementation can increase muscle protein synthesis to a greater disease 
A.

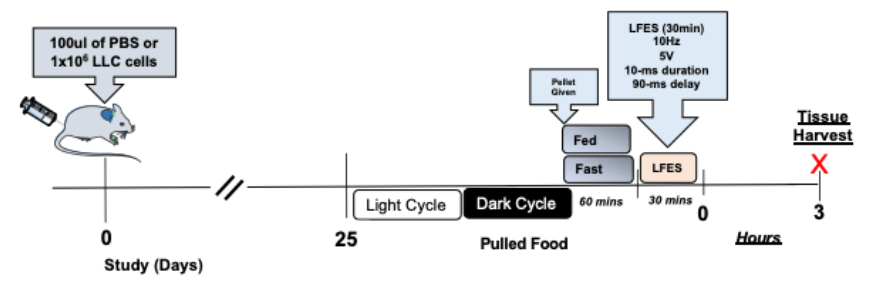

B.

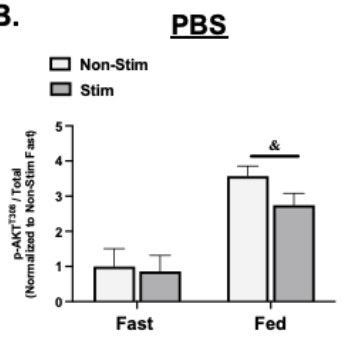

c.
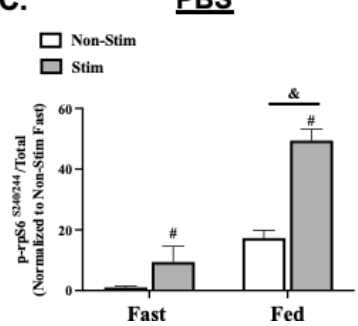

D.

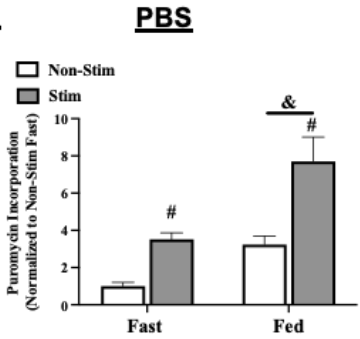

Figure 5-2. LFES and feeding exert synergistic effect on skeletal muscle protein synthesis in non-tumor-bearing mice

Notes: Data are presented as mean \pm SEM. A. Study Design. B. Phospho-AKT to total ratio in PBS fast and fed mice 3 hrs after LFES. C. Phospho-rpS6 to total ratio in PBS fast and fed mice 3 hrs after LFES. D. Puromycin incorporation in PBS fast and fed mice 3 hrs after LFES. Non-Stim leg was used as control. Two-way repeated measures ANOVA were used to compare within animal of the non-stim to stim leg in PBS fast and fed mice. Abbreviations: PBS: phosphate buffered saline. \# Main effect of Stim, \& Main effect of feeding. 
A.

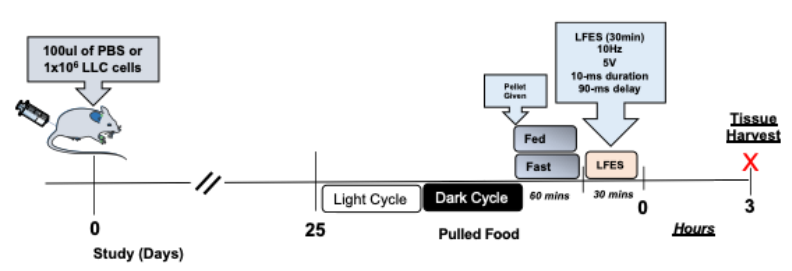

B.

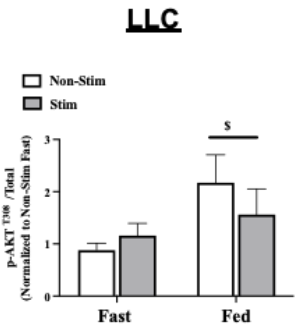

C.

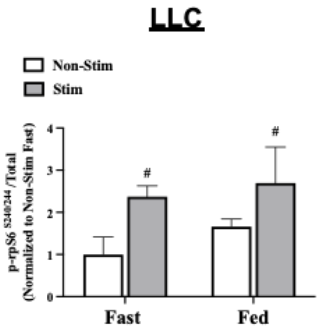

D.

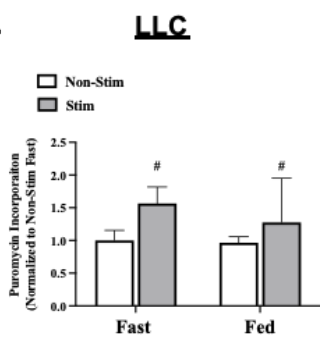

Figure 5-3. LLC inhibits the synergistic effect of LFES and feeding on skeletal muscle protein synthesis in tumor-bearing mice

Notes: Data are presented as mean \pm SEM. A. Study Design. B. Phospho-AKT to total ratio in LLC fast and fed mice 3 hrs after LFES. C. Phospho-rpS6 to total ratio in LLC fast and fed mice 3 hrs after LFES. D. Puromycin incorporation in PBS fast and fed mice 3 hrs after LFES. Non-Stim leg was used as control. Two-way repeated measures ANOVA were used to compare within animal of the non-stim to stim leg in LLC fast and fed mice. Abbreviations: PBS: phosphate buffered saline. \# Main effect of Stim, \& Main effect of feeding. 
A.

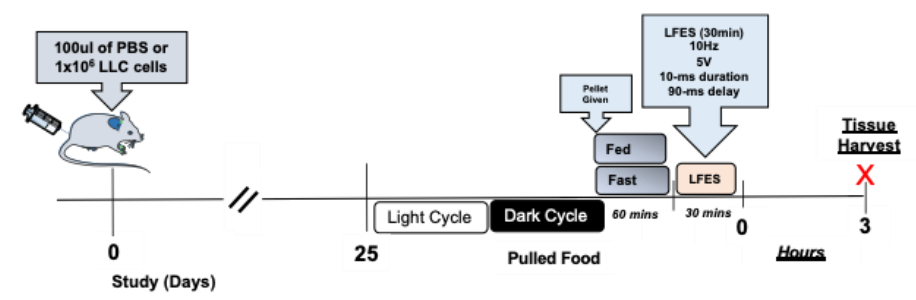

B.

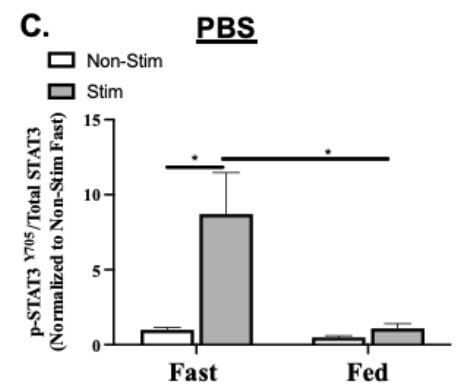

D.

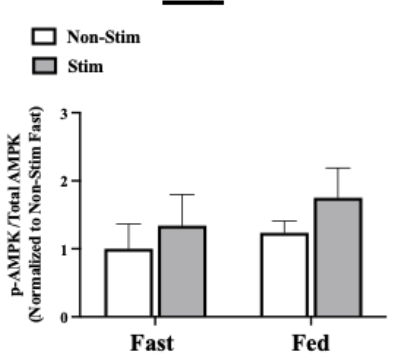

C.

LLCC

E. 느
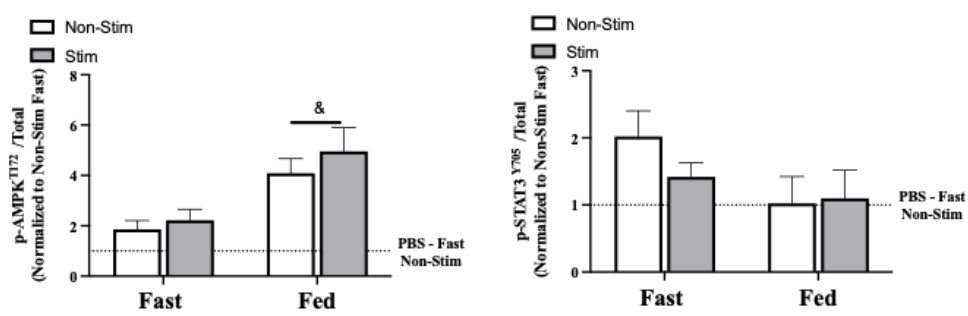

Figure 5-4. LLC tumor-bearing mice have altered feeding and contraction regulation of AMPK and STAT3

Notes: Data are presented as mean \pm SEM. A. Study Design. B. Phospho-AMPK to total ratio in PBS fast and fed mice 3 hrs after LFES. C. Phopsho-STAT3 to total ratio in PBS fast and fed mice 3 hrs after LFES. D. Phospho-AMPK to total ratio in LLC fast and fed mice 3 hrs after LFES. E. Phospho-STAT3 to total ratio in LLC fast and fed mice $3 \mathrm{hrs}$ after LFES. Non-Stim leg was used as control. Two-way repeated measures ANOVA were used to compare within animal of the non-stim to stim leg in PBS or LLC fast and fed mice. Abbreviations: PBS: phosphate buffered saline. \& Main effect of feeding. *Groups are different $3 \mathrm{hrs}$ post contraction. 
population. We extend previous studies by highlighting that pre-cachectic tumor-bearing mice can increase protein synthesis in response to LFES. However, protein synthesis was overall suppressed in tumor-bearing mice. We report the novel finding that the added protein synthesis response to feeding and contraction is blunted in pre-cachectic tumorbearing mice. Furthermore, we show that LFES was not sufficient to increase PGC1 $\alpha$ in pre-cachectic tumor-bearing mice. Taken together, LLC tumor-bearing mice exhibit suppressed anabolic signaling and do not synergistically respond to feeding and contraction.

Healthy skeletal muscle stimulates protein synthesis in response to anabolic stimuli associated with daily living, which can include physical activity and feeding (Egan \& Zierath, 2013). While basal muscle protein synthesis and mTORC1 signaling is suppressed in tumor-bearing mice the capacity for cachectic muscle to respond to an anabolic stimulus is not well understood. The inability of severely cachectic mice to perform voluntary exercise remains a consistent barrier and has limited our understanding of the muscle's response to exercise during refractory cachexia. To address this, we have reported that cachexia disrupts the metabolic and anabolic signaling response to a single bout of stimulated low-frequency concentric contractions which mimics low intensity, endurance type exercise. We extend these findings highlighting that pre-cachectic tumorbearing mice exhibit blunted anabolic response to LFES. These findings have significant implications because we report an altered response to contraction, prior to cachexia development. It can be hypothesized, that increased physical activity might play a beneficial role in improving the contraction response however this requires further investigation. Additionally, it has been suggested that an exercise threshold is required to improve skeletal muscle function during cachexia (Vanderveen et al., 2020). While we show the reduction in anabolic signaling in response to LFES in pre-cachectic mice, it is within reason to speculate that a more robust stimuli (high frequency electrical stimulation) is necessary to provide an anabolic response similar to that of non-tumor controls. Future studies are needed to determine if repeated bouts of low frequency electrical stimulation are sufficient to improve the basal suppression of protein synthesis. In addition, in aim 1 we show that feeding is sufficient to increase protein synthesis, however herein we do not in LLC tumor-bearing mice. The difference between the two studies is the time point post feeding (aim 1 was $1 \mathrm{hr}$ and aim 3 was $3.5 \mathrm{hrs}$ ). It is interesting to speculate that the anabolic window of feeding is shortened in pre-cachectic mice. However, future studies should determine if cachexia progression alters the anabolic window following feeding.

Contractile decrements have also been reported in cachectic muscle (Christensen et al., 2014; Roberts, Frye, et al., 2013), and is highly important given that force production and fatigue are directly related to life quality in disease (al-Majid \& McCarthy, 2001; Barreiro \& Gea, 2015; Siegel, 1989). We and others have recently shown that cachectic skeletal muscle develops a slow-fatigable contractile phenotype during cancer cachexia progression (Murphy et al., 2012; Roberts, Frye, et al., 2013; VanderVeen et al., 2018). Furthermore, the regulation of muscle's contractile properties are strongly related to the muscle's inflammatory signaling highlighting the regulation of muscle contraction by inflammation during disease (VanderVeen et al., 2019). 
Interestingly, cachectic muscle exhibits increased SERCA1 and calsequesterin mRNA in cachectic skeletal muscle (VanderVeen et al., 2018), further supporting the transition to a more faster muscle phenotype (Khodabukus \& Baar, 2015). While fatigue and weakness are the most commonly reported issues in cancer patients, we have only scratched the surface on trying to understand how this may occur (Chang et al., 2000). It is very possible that LLC tumor-bearing mice have impaired contractile properties which is inhibiting the ability to return protein synthesis levels to healthy control levels. While pre-cachectic tumor-bearing mice do exhibit slight fatiguability (VanderVeen et al., 2018), herein pre-cachectic tumor-bearing mice have the capacity to responds to contraction. Future studies should determine if the acute contraction response (immediately post) is dysregulated in pre-cachectic tumor-bearing mice.

A delimitation of this study is that we are examining the muscle's response after an acute bout of muscle contraction. A limitation of this aim is that we did not complete repeated contractions which can result in an exercise response. However, examining the acute contraction response is a necessary step in understanding the muscle's response to contraction during cancer cachexia progression, and is classical methodology for examining muscle's capacity to respond. While we did report a blunted response to contraction in tumor-bearing mice, it is possible that the stimulation protocol was not a strong enough stimulus. Future studies should determine if the response to LFES and HFES is similar in pre-cachectic tumor-bearing mice. These would provide interesting finding within itself because it means there is likely a stimulus threshold required. An alternative approach to stimulation is treadmill training or wheel access, however the voluntary nature of treadmill training and wheel activity is a confounder in tumor-bearing models because as cachexia progresses mice significantly reduce volitional activity.

\section{Conclusion}

In conclusion, we report that pre-cachectic tumor-bearing mice have the capacity to increase mTORC1 signaling and protein synthesis following an acute bout of low frequency electrical stimulation. However, pre-cachectic tumor-bearing mice do not have the capacity to synergistically increase protein synthesis by feeding and contraction. Future studies should determine if repeated low frequency contractions can improve the basal suppression of protein synthesis, and if repeated contractions can improve the synergistic effect of acute feeding and contraction. Additionally, future studies should also determine if the there is a stimulus threshold required to synergistically induce protein synthesis early during cachexia's progression 


\section{CHAPTER 6. DISCUSSION AND FUTURE DIRECTIONS}

\section{Discussion}

Cancer-induced cachexia disrupts skeletal muscle protein turnover in the basal condition (decreasing protein synthesis and increasing protein degradation). In preclinical models, our laboratory has demonstrated altered protein turnover through the suppression of anabolic signaling via mTORC1 which coincided with the chronic activation of AMPK (White, Baynes, et al., 2011). Interestingly, mTORC1 signaling exhibits diurnal fluctuations in response to changes throughout the day in feeding and activity (Counts et al., 2020). Furthermore, the cachectic environment disrupts these diurnal fluctuations, and suggest a link between disruptions in feeding and activity behaviors to the progression of cachexia and altered mTORC1 signaling (Counts et al., 2020). While progress has been made in understanding some of the mechanisms underlying the suppressed anabolic signaling in cachectic muscle, gaps remain in our understanding of muscle's ability to respond to anabolic stimuli prior to cachexia development. The purpose of this study was to determine if cachexia progression disrupts the feeding regulation of AMPK signaling and if gp130 signaling and muscle contraction can regulate this process. In Aim 1 we examined the feeding regulation of skeletal muscle protein synthesis in pre-cachectic tumor-bearing mice. Our results are in partial agreement with our hypothesis. In agreement with our hypothesis, pre-cachectic tumorbearing mice have overall suppressed Akt/mTORC1 signaling and protein synthesis. We hypothesized that pre-cachexia would inhibit the feeding response, however this did not happen. We reported the novel finding that pre-cachectic tumor-bearing mice have the capacity to increase muscle Akt/mTORC1 signaling and protein synthesis, and lower AMPK signaling in response to feeding. Lastly, in agreement with previous findings reporting AMPK's regulation of cachectic muscle signaling, muscle-specific AMPK loss was sufficient to improve the fasting suppression of muscle mTORC1 and protein synthesis in pre-cachectic tumor-bearing mice.

Inflammation has been implicated as a key mediator of cancer-induced cachexia leading to chronic elevation of skeletal muscle STAT3 and MAPK activity and may be critical for gp130 activation (Deans \& Wigmore, 2005). Glycoprotein-130 (gp130) is a regulatory point of interaction between systemic inflammation and JAK/STAT and MAPK muscle signaling (White \& Stephens, 2011). We have previously reported muscle gp130 loss prevented muscle wasting and restored STAT3 and MAPK signaling (Puppa, Gao, et al., 2014). In pre-clinical models, our laboratory has demonstrated altered protein turnover through the suppression of anabolic signaling via mTORC1 which coincided with the chronic activation of AMPK (White, Baynes, et al., 2011). In aim 2, we examined if muscle gp130 signaling regulates the feeding regulation of AMPK during cancer cachexia progression. We hypothesized that gp130 regulates AMPK's regulation of feeding. While we report muscle gp130 loss lowered the fasting induction of AMPK in pre-cachectic tumor-bearing mice, gp130 loss was not sufficient to improve muscle protein synthesis. Furthermore, muscle gp130 loss did not alter the feeding regulation of muscle Akt/mTORC1 signaling and protein synthesis in pre-cachectic tumor-bearing 
mice. Taken together, gp130's regulation in pre-cachectic tumor-bearing mice does not exert regulation on skeletal muscle protein synthesis. Given the known regulation of gp130 and associated signaling on muscle wasting, our findings suggest that gp130's regulation of skeletal muscle's response to anabolic stimuli is likely more important in cachectic muscle.

Cancer cachexia exhibits reduced volitional activity, whole body weakness, and fatigue in cancer patients and in pre-clinical models (Baltgalvis et al., 2010; Murphy et al., 2012; Toth et al., 2016). Exercise has been hypothesized to be a potential therapeutic option for cancer induced cachexia (Hardee et al., 2019). We have previously shown that an acute bout of low-frequency electrical stimulation (LFES) induced downstream mTORC1 phosphorylation and oxidative capacity, however this response was suppressed in cachectic muscle (Puppa, Murphy, et al., 2014). In aim 3, we examined if an acute bout of muscle contraction could improve muscle protein synthesis response to feeding during the progression of cachexia. We hypothesized that an acute bout of muscle contraction could improve muscle protein synthesis response to feeding and would be greater during pre-cachexia compared to refractory cachexia. Our results partially support our hypothesis. We found that pre-cachectic tumor-bearing mice exhibit suppressed protein synthesis in response to low frequency electrical stimulation. Furthermore, we hypothesized a synergistic effect of feeding and contraction would be present in precachectic tumor-bearing mice, however pre-cachectic tumor-bearing mice were unable to synergistically induce protein synthesis in response to feeding and contraction. Unfortunately, we set out to examine the range of cachexia progression, but our mice were pre-cachectic which does not allow us to examine the progression of cachexia, however this does provide useful information highlighting that the blunted contraction response occurs prior to wasting.

Overall, pre-cachectic tumor-bearing mice have suppressed Akt/mTORC1 signaling and protein synthesis in the fast and fed condition. Muscle-specific AMPK loss was sufficient to improve muscle mTORC1 and protein synthesis in pre-cachectic tumorbearing mice. Gp130 can regulate the fasting induction of AMPK, without improving downstream protein synthesis in pre-cachectic tumor-bearing mice. Tumor-bearing mice do not have the capacity to synergistically increase mTORC1 signaling and protein synthesis in response to feeding and contraction. In summary, feeding can induce Akt/mTORC1 and protein synthesis, which is not regulated by gp130 in pre-cachectic tumor-bearing mice. AMPK regulates the fasting suppression of protein synthesis in tumor-bearing mice. The added protein synthesis response to feeding and contraction is blunted in pre-cachectic tumor-bearing mice. These findings provide novel insight into the regulation of Akt/mTORC1 signaling and protein synthesis in response to feeding. Additionally, these studies provide novel understanding of gp130's regulation of AMPK prior to cachexia development, and the blunted anabolic muscle response to an acute bout of muscle contractions. By understanding these intracellular signaling processes and perturbations prior to cachexia development, we will be able to elucidate potential therapeutic targets and treatment options to manipulate and prevent cancer cachexia 


\section{Future Directions}

While we have made several advances in understanding the basal regulation of skeletal muscle during cancer cachexia progression and cachectic muscle's capacity to respond to anabolic stimuli several questions still arise. We report the novel finding that feeding has the capacity to increase muscle Akt/mTORC1 signaling and protein synthesis, and lower AMPK in pre-cachectic tumor-bearing mice. Importantly, precachectic tumor-bearing mice have overall suppressed Akt/mTORC1 signaling and protein synthesis independent of the fast and fed condition. Future, studies should examine if cachectic muscle has the capacity to increase muscle Akt/mTORC1 signaling and protein synthesis. We also report the novel finding that muscle-specific AMPK loss was sufficient to improve the fasting suppression of protein synthesis in pre-cachectic tumor-bearing mice, however the addition of feeding was not sufficient to further increase protein synthesis. Further work is required to determine why muscle AMPK loss in combination with feeding did not restore impaired muscle protein synthesis in precachectic tumor-bearing mice. Additionally, we extend previous studies by highlighting that muscle gp130 loss reduced elevated AMPK but was not sufficient to rescue suppressed protein synthesis in pre-cachectic tumor-bearing mice. Future studies are warranted to examine if gp130 loss is sufficient to suppress AMPK and rescue muscle protein synthesis in cachectic muscle, specifically when the IL-6 family of cytokines are elevated. Furthermore, given that we have previously found AMPK loss was sufficient to rescue protein synthesis in tumor-bearing mice, future studies are warranted to determine why gp130 loss can reduce AMPK, but is insufficient to improve protein synthesis in tumor-bearing mice. Previous studies have shown that muscle gp130 loss can prevent cachexia development, suggesting that targeting gp130 in cachectic muscle might provide difficulty. Therefore, future studies could examine if STAT3 inhibition in cachectic muscle can improve the fasting regulation of AMPK and protein synthesis. Lastly, we extend previous studies by demonstrating that pre-cachectic tumor-bearing mice exhibit suppressed mTORC1 signaling and protein synthesis in response to an acute bout lowfrequency electrical stimulation. We report the novel finding that the synergistic increase of mTORC1 signaling and protein synthesis in response to feeding and contraction is inhibited in pre-cachectic tumor-bearing mice. Future studies are warranted to examine if repeated contractions can improve the acute response to feeding and contraction.

Furthermore, future studies are warranted to examine if repeated contractions can improve the fasting suppression of protein synthesis during cancer cachexia progression. Finally, researchers should examine if AMPK is inhibiting the protein synthesis response to contraction during cancer cachexia progression 


\section{LIST OF REFERENCES}

Abou Sawan, S., van Vliet, S., Parel, J. T., Beals, J. W., Mazzulla, M., West, D. W. D., . . .Moore, D. R. (2018). Translocation and protein complex co-localization of mTOR is associated with postprandial myofibrillar protein synthesis at rest and after endurance exercise. Physiol Rep, 6(5). https://doi.org/10.14814/phy2.13628

Abraham, R. T. (2010). Lysosomal Rag-ulation of mTOR complex 1 activity. Cell Metab, 11(5), 341-342. https://doi.org/10.1016/j.cmet.2010.04.010

al-Majid, S., \& McCarthy, D. O. (2001). Cancer-induced fatigue and skeletal muscle wasting: the role of exercise. Biol Res Nurs, 2(3), 186-197. https://doi.org/10.1177/109980040100200304

Ali, S., Chen, J. A., \& Garcia, J. M. (2013). Clinical development of ghrelin axis-derived molecules for cancer cachexia treatment. Curr Opin Support Palliat Care, 7(4), 368-375. https://doi.org/10.1097/SPC.0000000000000012

Alves, C. R. R., MacDonald, T. L., Nigro, P., Pathak, P., Hirshman, M. F., Goodyear, L. J., \& Lessard, S. J. (2019). Reduced sucrose nonfermenting AMPK-related kinase (SNARK) activity aggravates cancer-induced skeletal muscle wasting. Biomed Pharmacother, 117, 109197. https://doi.org/10.1016/j.biopha.2019.109197

Anderson, L. J., Albrecht, E. D., \& Garcia, J. M. (2017). Update on Management of Cancer-Related Cachexia. Curr Oncol Rep, 19(1), 3. https://doi.org/10.1007/s11912-017-0562-0

Andratsch, M., Mair, N., Constantin, C. E., Scherbakov, N., Benetti, C., Quarta, S., ... Kress, M. (2009). A key role for gp130 expressed on peripheral sensory nerves in pathological pain. J Neurosci, 29(43), 13473-13483.

https://doi.org/10.1523/JNEUROSCI.1822-09.2009

Antoun, S., \& Raynard, B. (2018). Muscle protein anabolism in advanced cancer patients: response to protein and amino acids support, and to physical activity. Ann Oncol, 29(suppl_2), ii10-ii17. https://doi.org/10.1093/annonc/mdx809

Argiles, J. M., Lopez-Soriano, F. J., \& Busquets, S. (2015). Muscle wasting in cancer: the role of mitochondria. Curr Opin Clin Nutr Metab Care, 18(3), 221-225. https://doi.org/10.1097/MCO.0000000000000164

Attardi, G., \& Schatz, G. (1988). Biogenesis of mitochondria. Аnпи Rev Cell Biol, 4, 289333. https://doi.org/10.1146/annurev.cb.04.110188.001445

Aversa, Z., Pin, F., Lucia, S., Penna, F., Verzaro, R., Fazi, M., . . Muscaritoli, M. (2016). Autophagy is induced in the skeletal muscle of cachectic cancer patients. Scientific Reports, 6. https://doi.org/ARTN 30340

10.1038/srep30340

Baar, K., \& Esser, K. (1999). Phosphorylation of p70(S6k) correlates with increased skeletal muscle mass following resistance exercise. Am J Physiol, 276(1), C120127. https://doi.org/10.1152/ajpcell.1999.276.1.C120

Baar, K., Wende, A. R., Jones, T. E., Marison, M., Nolte, L. A., Chen, M., . . Holloszy, J. O. (2002). Adaptations of skeletal muscle to exercise: rapid increase in the transcriptional coactivator PGC-1. FASEB J, 16(14), 1879-1886. https://doi.org/10.1096/fj.02-0367com 
Bagherniya, M., Butler, A. E., Barreto, G. E., \& Sahebkar, A. (2018). The effect of fasting or calorie restriction on autophagy induction: A review of the literature. Ageing Res Rev, 47, 183-197. https://doi.org/10.1016/j.arr.2018.08.004

Bajaj, L., Lotfi, P., Pal, R., Ronza, A. D., Sharma, J., \& Sardiello, M. (2019). Lysosome biogenesis in health and disease. J Neurochem, 148(5), 573-589. https://doi.org/10.1111/jnc. 14564

Ballaro, R., Costelli, P., \& Penna, F. (2016). Animal models for cancer cachexia. Curr Opin Support Palliat Care, 10(4), 281-287. https://doi.org/10.1097/SPC.0000000000000233

Balnis, J., Korponay, T. C., \& Jaitovich, A. (2020). AMP-Activated Protein Kinase (AMPK) at the Crossroads Between CO2 Retention and Skeletal Muscle Dysfunction in Chronic Obstructive Pulmonary Disease (COPD). Int J Mol Sci, 21(3). https://doi.org/10.3390/ijms21030955

Baltgalvis, K. A., Berger, F. G., Pena, M. M., Davis, J. M., White, J. P., \& Carson, J. A. (2009). Muscle wasting and interleukin-6-induced atrogin-I expression in the cachectic Apc ( Min/+ ) mouse. Pflugers Arch, 457(5), 989-1001. https://doi.org/10.1007/s00424-008-0574-6

Baltgalvis, K. A., Berger, F. G., Pena, M. M., Mark Davis, J., White, J. P., \& Carson, J. A. (2010). Activity level, apoptosis, and development of cachexia in Apc(Min/+) mice. J Appl Physiol (1985), 109(4), 1155-1161. https://doi.org/10.1152/japplphysiol.00442.2010

Bar-Peled, L., \& Sabatini, D. M. (2014). Regulation of mTORC1 by amino acids. Trends Cell Biol, 24(7), 400-406. https://doi.org/10.1016/j.tcb.2014.03.003

Baracos, V. E. (2000). Regulation of skeletal-muscle-protein turnover in cancerassociated cachexia. Nutrition, 16(10), 1015-1018. https://www.ncbi.nlm.nih.gov/pubmed/11054610

Baracos, V. E. (2018). Bridging the gap: are animal models consistent with clinical cancer cachexia? Nat Rev Clin Oncol, 15(4), 197-198. https://doi.org/10.1038/nrclinonc.2018.14

Baracos, V. E., Martin, L., Korc, M., Guttridge, D. C., \& Fearon, K. C. H. (2018). Cancer-associated cachexia. Nat Rev Dis Primers, 4, 17105. https://doi.org/10.1038/nrdp.2017.105

Baracos, V. E., Mazurak, V. C., \& Bhullar, A. S. (2019). Cancer cachexia is defined by an ongoing loss of skeletal muscle mass. Ann Palliat Med, 8(1), 3-12. https://doi.org/10.21037/apm.2018.12.01

Barclay, R. D., Burd, N. A., Tyler, C., Tillin, N. A., \& Mackenzie, R. W. (2019). The Role of the IGF-1 Signaling Cascade in Muscle Protein Synthesis and Anabolic Resistance in Aging Skeletal Muscle. Front Nutr, 6, 146. https://doi.org/10.3389/fnut.2019.00146

Barnes, B. R., Marklund, S., Steiler, T. L., Walter, M., Hjalm, G., Amarger, V., . . . Andersson, L. (2004). The 5'-AMP-activated protein kinase gamma3 isoform has a key role in carbohydrate and lipid metabolism in glycolytic skeletal muscle. $J$ Biol Chem, 279(37), 38441-38447. https://doi.org/10.1074/jbc.M405533200

Barreiro, E., \& Gea, J. (2015). Respiratory and Limb Muscle Dysfunction in COPD. COPD, 12(4), 413-426. https://doi.org/10.3109/15412555.2014.974737 
Beavers, K. M., Brinkley, T. E., \& Nicklas, B. J. (2010). Effect of exercise training on chronic inflammation. Clin Chim Acta, 411(11-12), 785-793.

https://doi.org/10.1016/j.cca.2010.02.069

Beck Jorgensen, S., O'Neill, H. M., Hewitt, K., Kemp, B. E., \& Steinberg, G. R. (2009). Reduced AMP-activated protein kinase activity in mouse skeletal muscle does not exacerbate the development of insulin resistance with obesity. Diabetologia, 52(11), 2395-2404. https://doi.org/10.1007/s00125-009-1483-8

Bell, R. A., Al-Khalaf, M., \& Megeney, L. A. (2016). The beneficial role of proteolysis in skeletal muscle growth and stress adaptation. Skelet Muscle, 6, 16. https://doi.org/10.1186/s13395-016-0086-6

Benard, G., \& Karbowski, M. (2009). Mitochondrial fusion and division: Regulation and role in cell viability. Semin Cell Dev Biol, 20(3), 365-374. https://doi.org/10.1016/j.semcdb.2008.12.012

Bentzinger, C. F., Romanino, K., Cloetta, D., Lin, S., Mascarenhas, J. B., Oliveri, F., . . . Ruegg, M. A. (2008). Skeletal muscle-specific ablation of raptor, but not of rictor, causes metabolic changes and results in muscle dystrophy. Cell Metab, 8(5), 411424. https://doi.org/10.1016/j.cmet.2008.10.002

Betz, U. A., Bloch, W., van den Broek, M., Yoshida, K., Taga, T., Kishimoto, T., . . . Muller, W. (1998). Postnatally induced inactivation of gp130 in mice results in neurological, cardiac, hematopoietic, immunological, hepatic, and pulmonary defects. J Exp Med, 188(10), 1955-1965. https://doi.org/10.1084/jem.188.10.1955

Bird, S. R., \& Hawley, J. A. (2016). Update on the effects of physical activity on insulin sensitivity in humans. BMJ Open Sport Exerc Med, 2(1), e000143. https://doi.org/10.1136/bmjsem-2016-000143

Birk, J. B., \& Wojtaszewski, J. F. (2006). Predominant alpha2/beta2/gamma3 AMPK activation during exercise in human skeletal muscle. J Physiol, 577(Pt 3), 10211032. https://doi.org/10.1113/jphysiol.2006.120972

Bolster, D. R., Crozier, S. J., Kimball, S. R., \& Jefferson, L. S. (2002). AMP-activated protein kinase suppresses protein synthesis in rat skeletal muscle through downregulated mammalian target of rapamycin (mTOR) signaling. J Biol Chem, 277(27), 23977-23980. https://doi.org/10.1074/jbc.C200171200

Bonaldo, P., \& Sandri, M. (2013). Cellular and molecular mechanisms of muscle atrophy. Dis Model Mech, 6(1), 25-39. https://doi.org/10.1242/dmm.010389

Bonetto, A., Aydogdu, T., Jin, X., Zhang, Z., Zhan, R., Puzis, L., . . . Zimmers, T. A. (2012). JAK/STAT3 pathway inhibition blocks skeletal muscle wasting downstream of IL-6 and in experimental cancer cachexia. Am J Physiol Endocrinol Metab, 303(3), E410-421. https://doi.org/10.1152/ajpendo.00039.2012

Bonetto, A., Kays, J. K., Parker, V. A., Matthews, R. R., Barreto, R., Puppa, M. J., . . . Zimmers, T. A. (2016). Differential Bone Loss in Mouse Models of Colon Cancer Cachexia. Front Physiol, 7, 679. https://doi.org/10.3389/fphys.2016.00679

Bonetto, A., Rupert, J. E., Barreto, R., \& Zimmers, T. A. (2016). The Colon-26 Carcinoma Tumor-bearing Mouse as a Model for the Study of Cancer Cachexia. $J$ Vis $\operatorname{Exp}(117)$. https://doi.org/10.3791/54893

Bossola, M., Muscaritoli, M., Costelli, P., Grieco, G., Bonelli, G., Pacelli, F., . . . Baccino, F. M. (2003). Increased muscle proteasome activity correlates with 
disease severity in gastric cancer patients. Ann Surg, 237(3), 384-389.

https://doi.org/10.1097/01.SLA.0000055225.96357.71

Boucher, J., Kleinridders, A., \& Kahn, C. R. (2014). Insulin receptor signaling in normal and insulin-resistant states. Cold Spring Harb Perspect Biol, 6(1). https://doi.org/10.1101/cshperspect.a009191

Brandauer, J., Vienberg, S. G., Andersen, M. A., Ringholm, S., Risis, S., Larsen, P. S., . . . Treebak, J. T. (2013). AMP-activated protein kinase regulates nicotinamide phosphoribosyl transferase expression in skeletal muscle. J Physiol, 591(20), 5207-5220. https://doi.org/10.1113/jphysiol.2013.259515

Breen, L., \& Phillips, S. M. (2011). Skeletal muscle protein metabolism in the elderly: Interventions to counteract the 'anabolic resistance' of ageing. Nutr Metab (Lond), 8, 68. https://doi.org/10.1186/1743-7075-8-68

Breen, L., \& Phillips, S. M. (2013). Interactions between exercise and nutrition to prevent muscle waste during ageing. Br J Clin Pharmacol, 75(3), 708-715. https://doi.org/10.1111/j.1365-2125.2012.04456.x

Brook, M. S., Wilkinson, D. J., \& Atherton, P. J. (2017). Nutrient modulation in the management of disease-induced muscle wasting: evidence from human studies. Curr Opin Clin Nutr Metab Care, 20(6), 433-439. https://doi.org/10.1097/MCO.0000000000000413

Brown, J. L., Rosa-Caldwell, M. E., Lee, D. E., Blackwell, T. A., Brown, L. A., Perry, R. A., ... Greene, N. P. (2017). Mitochondrial degeneration precedes the development of muscle atrophy in progression of cancer cachexia in tumourbearing mice. J Cachexia Sarcopenia Muscle, 8(6), 926-938. https://doi.org/10.1002/jcsm.12232

Brozinick, J. T., Jr., Etgen, G. J., Jr., Yaspelkis, B. B., 3rd, \& Ivy, J. L. (1992). Contraction-activated glucose uptake is normal in insulin-resistant muscle of the obese Zucker rat. J Appl Physiol (1985), 73(1), 382-387. https://doi.org/10.1152/jappl.1992.73.1.382

Bujak, A. L., Crane, J. D., Lally, J. S., Ford, R. J., Kang, S. J., Rebalka, I. A., . . . Steinberg, G. R. (2015). AMPK activation of muscle autophagy prevents fastinginduced hypoglycemia and myopathy during aging. Cell Metab, 21(6), 883-890. https://doi.org/10.1016/j.cmet.2015.05.016

Burd, N. A., Tang, J. E., Moore, D. R., \& Phillips, S. M. (2009). Exercise training and protein metabolism: influences of contraction, protein intake, and sex-based differences. J Appl Physiol (1985), 106(5), 1692-1701. https://doi.org/10.1152/japplphysiol.91351.2008

Bye, A., Sjoblom, B., Wentzel-Larsen, T., Gronberg, B. H., Baracos, V. E., Hjermstad, M. J., . . Jordhoy, M. (2017). Muscle mass and association to quality of life in non-small cell lung cancer patients. J Cachexia Sarcopenia Muscle, 8(5), 759767. https://doi.org/10.1002/jcsm.12206

Cai, D., Frantz, J. D., Tawa, N. E., Jr., Melendez, P. A., Oh, B. C., Lidov, H. G., . . Shoelson, S. E. (2004). IKKbeta/NF-kappaB activation causes severe muscle wasting in mice. Cell, 119(2), 285-298. https://doi.org/10.1016/j.cell.2004.09.027

Canto, C., Jiang, L. Q., Deshmukh, A. S., Mataki, C., Coste, A., Lagouge, M., . . . Auwerx, J. (2010). Interdependence of AMPK and SIRT1 for metabolic 
adaptation to fasting and exercise in skeletal muscle. Cell Metab, 11(3), 213-219. https://doi.org/10.1016/j.cmet.2010.02.006

Chan, D. C. (2020). Mitochondrial Dynamics and Its Involvement in Disease. Annu Rev Pathol, 15, 235-259. https://doi.org/10.1146/annurev-pathmechdis-012419$\underline{032711}$

Chang, V. T., Hwang, S. S., Feuerman, M., \& Kasimis, B. S. (2000). Symptom and quality of life survey of medical oncology patients at a veterans affairs medical center: a role for symptom assessment. Cancer, 88(5), 1175-1183. https://doi.org/10.1002/(sici)1097-0142(20000301)88:5<1175::aidcncr30>3.0.co;2-n

Chen, H., \& Chan, D. C. (2010). Physiological functions of mitochondrial fusion. Ann N Y Acad Sci, 1201, 21-25. https://doi.org/10.1111/j.1749-6632.2010.05615.x

Chen, J. A., Splenser, A., Guillory, B., Luo, J., Mendiratta, M., Belinova, B., . . Garcia, J. M. (2015). Ghrelin prevents tumour- and cisplatin-induced muscle wasting: characterization of multiple mechanisms involved. J Cachexia Sarcopenia Muscle, 6(2), 132-143. https://doi.org/10.1002/jcsm.12023

Chiappalupi, S., Sorci, G., Vukasinovic, A., Salvadori, L., Sagheddu, R., Coletti, D., . . . Riuzzi, F. (2020). Targeting RAGE prevents muscle wasting and prolongs survival in cancer cachexia. J Cachexia Sarcopenia Muscle, 11(4), 929-946. https://doi.org/10.1002/jcsm.12561

Cho, K. M., Park, H., Oh, D. Y., Kim, T. Y., Lee, K. H., Han, S. W., . . B Bang, Y. J. (2017). Skeletal muscle depletion predicts survival of patients with advanced biliary tract cancer undergoing palliative chemotherapy. Oncotarget, 8(45), 79441-79452. https://doi.org/10.18632/oncotarget.18345

Christensen, J. F., Jones, L. W., Andersen, J. L., Daugaard, G., Rorth, M., \& Hojman, P. (2014). Muscle dysfunction in cancer patients. Ann Oncol, 25(5), 947-958. https://doi.org/10.1093/annonc/mdt551

Ciciliot, S., Rossi, A. C., Dyar, K. A., Blaauw, B., \& Schiaffino, S. (2013). Muscle type and fiber type specificity in muscle wasting. Int J Biochem Cell Biol, 45(10), 2191-2199. https://doi.org/10.1016/j.biocel.2013.05.016

Cole, C. L., Kleckner, I. R., Jatoi, A., Schwarz, E. M., \& Dunne, R. F. (2018). The Role of Systemic Inflammation in Cancer-Associated Muscle Wasting and Rationale for Exercise as a Therapeutic Intervention. JCSM Clin Rep, 3(2). https://www.ncbi.nlm.nih.gov/pubmed/31134216

Coletti, D., Aulino, P., Pigna, E., Barteri, F., Moresi, V., Annibali, D., . . Berardi, E. (2016). Spontaneous Physical Activity Downregulates Pax7 in Cancer Cachexia. Stem Cells Int, 2016, 6729268. https://doi.org/10.1155/2016/6729268

Counts, B. R., Halle, J. L., \& Carson, J. A. (2021). Early Onset Physical Inactivity and Metabolic Dysfunction in Tumor-bearing Mice Is Associated with Accelerated Cachexia. Med Sci Sports Exerc. https://doi.org/10.1249/MSS.0000000000002772

Counts, B. R., Hardee, J. P., Fix, D. K., Vanderveen, B. N., Montalvo, R. N., \& Carson, J. A. (2020). Cachexia Disrupts Diurnal Regulation of Activity, Feeding, and Muscle Mechanistic Target of Rapamycin Complex 1 in Mice. Med Sci Sports Exerc, 52(3), 577-587. https://doi.org/10.1249/MSS.0000000000002166 
Cunningham, J. T., Rodgers, J. T., Arlow, D. H., Vazquez, F., Mootha, V. K., \& Puigserver, P. (2007). mTOR controls mitochondrial oxidative function through a YY1-PGC-1alpha transcriptional complex. Nature, 450(7170), 736-740. https://doi.org/10.1038/nature06322

Daou, H. N. (2020). Exercise as an anti-inflammatory therapy for cancer cachexia: a focus on interleukin-6 regulation. Am J Physiol Regul Integr Comp Physiol, 318(2), R296-R310. https://doi.org/10.1152/ajpregu.00147.2019

de Theije, C. C., Langen, R. C., Lamers, W. H., Gosker, H. R., Schols, A. M., \& Kohler, S. E. (2015). Differential sensitivity of oxidative and glycolytic muscles to hypoxia-induced muscle atrophy. J Appl Physiol (1985), 118(2), 200-211. https://doi.org/10.1152/japplphysiol.00624.2014

de Theije, C. C., Schols, A., Lamers, W. H., Neumann, D., Kohler, S. E., \& Langen, R. C. J. (2018). Hypoxia impairs adaptation of skeletal muscle protein turnover- and AMPK signaling during fasting-induced muscle atrophy. PLoS One, 13(9), e0203630. https://doi.org/10.1371/journal.pone.0203630

Deans, C., \& Wigmore, S. J. (2005). Systemic inflammation, cachexia and prognosis in patients with cancer. Curr Opin Clin Nutr Metab Care, 8(3), 265-269. https://doi.org/10.1097/01.mco.0000165004.93707.88

DeBerardinis, R. J., \& Thompson, C. B. (2012). Cellular metabolism and disease: what do metabolic outliers teach us? Cell, 148(6), 1132-1144. https://doi.org/10.1016/j.cell.2012.02.032

Dethlefsen, M. M., Bertholdt, L., Gudiksen, A., Stankiewicz, T., Bangsbo, J., van Hall, G., . . Pilegaard, H. (2018). Training state and skeletal muscle autophagy in response to 36 h of fasting. J Appl Physiol (1985), 125(5), 1609-1619. https://doi.org/10.1152/japplphysiol.01146.2017

Deutz, N. E., Safar, A., Schutzler, S., Memelink, R., Ferrando, A., Spencer, H., . . Wolfe, R. R. (2011). Muscle protein synthesis in cancer patients can be stimulated with a specially formulated medical food. Clin Nutr, 30(6), 759-768. https://doi.org/10.1016/j.clnu.2011.05.008

Deval, C., Calonne, J., Coudy-Gandilhon, C., Vazeille, E., Bechet, D., Polge, C., . . . Combaret, L. (2020). Mitophagy and Mitochondria Biogenesis Are Differentially Induced in Rat Skeletal Muscles during Immobilization and/or Remobilization. Int J Mol Sci, 21(10). https://doi.org/10.3390/ijms21103691

Drogemuller, K., Helmuth, U., Brunn, A., Sakowicz-Burkiewicz, M., Gutmann, D. H., Mueller, W., ... Schluter, D. (2008). Astrocyte gp130 expression is critical for the control of Toxoplasma encephalitis. J Immunol, 181(4), 2683-2693. https://doi.org/10.4049/jimmunol.181.4.2683

Dumitru, A., Radu, B. M., Radu, M., \& Cretoiu, S. M. (2018). Muscle Changes During Atrophy. Adv Exp Med Biol, 1088, 73-92. https://doi.org/10.1007/978-981-13$\underline{1435-3 \quad 4}$

Dworzak, F., Ferrari, P., Gavazzi, C., Maiorana, C., \& Bozzetti, F. (1998). Effects of cachexia due to cancer on whole body and skeletal muscle protein turnover. Cancer, 82(1), 42-48. https://www.ncbi.nlm.nih.gov/pubmed/9428478

Eden, E., Ekman, L., Bennegard, K., Lindmark, L., \& Lundholm, K. (1984). Whole-body tyrosine flux in relation to energy expenditure in weight-losing cancer patients. Metabolism, 33(11), 1020-1027. https://doi.org/10.1016/0026-0495(84)90231-2 
Egan, B., \& Zierath, J. R. (2013). Exercise metabolism and the molecular regulation of skeletal muscle adaptation. Cell Metab, 17(2), 162-184.

https://doi.org/10.1016/j.cmet.2012.12.012

Emery, P. W., Edwards, R. H., Rennie, M. J., Souhami, R. L., \& Halliday, D. (1984).

Protein synthesis in muscle measured in vivo in cachectic patients with cancer. $\mathrm{Br}$

Med J (Clin Res Ed), 289(6445), 584-586. https://doi.org/10.1136/bmj.289.6445.584

Ernst, M., \& Jenkins, B. J. (2004). Acquiring signalling specificity from the cytokine receptor gp130. Trends Genet, 20(1), 23-32. https://doi.org/10.1016/j.tig.2003.11.003

Esper, D. H., \& Harb, W. A. (2005). The cancer cachexia syndrome: a review of metabolic and clinical manifestations. Nutr Clin Pract, 20(4), 369-376. https://doi.org/10.1177/0115426505020004369

Evans, W. J., Morley, J. E., Argiles, J., Bales, C., Baracos, V., Guttridge, D., . . Anker, S. D. (2008). Cachexia: a new definition. Clin Nutr, 27(6), 793-799. https://doi.org/10.1016/j.clnu.2008.06.013

Favaro, G., Romanello, V., Varanita, T., Andrea Desbats, M., Morbidoni, V., Tezze, C., . .. Sandri, M. (2019). DRP1-mediated mitochondrial shape controls calcium homeostasis and muscle mass. Nat Commun, 10(1), 2576. https://doi.org/10.1038/s41467-019-10226-9

Fayard, E., Tintignac, L. A., Baudry, A., \& Hemmings, B. A. (2005). Protein kinase B/Akt at a glance. J Cell Sci, 118(Pt 24), 5675-5678. https://doi.org/10.1242/jcs.02724

Fearon, K. C., Glass, D. J., \& Guttridge, D. C. (2012). Cancer cachexia: mediators, signaling, and metabolic pathways. Cell Metab, 16(2), 153-166. https://doi.org/10.1016/i.cmet.2012.06.011

Fearon, K. C., Von Meyenfeldt, M. F., Moses, A. G., Van Geenen, R., Roy, A., Gouma, D. J., . . . Tisdale, M. J. (2003). Effect of a protein and energy dense N-3 fatty acid enriched oral supplement on loss of weight and lean tissue in cancer cachexia: a randomised double blind trial. Gut, 52(10), 1479-1486. https://doi.org/10.1136/gut.52.10.1479

Fearon, K. C., Voss, A. C., Hustead, D. S., \& Cancer Cachexia Study, G. (2006). Definition of cancer cachexia: effect of weight loss, reduced food intake, and systemic inflammation on functional status and prognosis. Am J Clin Nutr, 83(6), 1345-1350. https://doi.org/10.1093/ajen/83.6.1345

Fentz, J., Kjobsted, R., Birk, J. B., Jordy, A. B., Jeppesen, J., Thorsen, K., . . . Wojtaszewski, J. F. (2015). AMPKalpha is critical for enhancing skeletal muscle fatty acid utilization during in vivo exercise in mice. FASEB J, 29(5), 1725-1738. https://doi.org/10.1096/fj.14-266650

Fermoselle, C., Garcia-Arumi, E., Puig-Vilanova, E., Andreu, A. L., Urtreger, A. J., de Kier Joffe, E. D., . . . Barreiro, E. (2013). Mitochondrial dysfunction and therapeutic approaches in respiratory and limb muscles of cancer cachectic mice. Exp Physiol, 98(9), 1349-1365. https://doi.org/10.1113/expphysiol.2013.072496

Fitts, R. H. (2008). The cross-bridge cycle and skeletal muscle fatigue. J Appl Physiol (1985), 104(2), 551-558. https://doi.org/10.1152/japplphysiol.01200.2007 
Fix, D. K., Counts, B. R., Smuder, A. J., Sarzynski, M. A., Koh, H. J., \& Carson, J. A. (2021). Wheel running improves fasting-induced AMPK signaling in skeletal muscle from tumor-bearing mice. Physiol Rep, 9(14), e14924. https://doi.org/10.14814/phy2.14924

Fix, D. K., Hardee, J. P., Gao, S., VanderVeen, B. N., Velazquez, K. T., \& Carson, J. A. (2018). Role of gp130 in basal and exercise-trained skeletal muscle mitochondrial quality control. J Appl Physiol (1985), 124(6), 1456-1470. https://doi.org/10.1152/japplphysiol.01063.2017

Fonseca, G., Farkas, J., Dora, E., von Haehling, S., \& Lainscak, M. (2020). Cancer Cachexia and Related Metabolic Dysfunction. Int J Mol Sci, 21(7). https://doi.org/10.3390/ijms21072321

Fouladiun, M., Korner, U., Gunnebo, L., Sixt-Ammilon, P., Bosaeus, I., \& Lundholm, K. (2007). Daily physical-rest activities in relation to nutritional state, metabolism, and quality of life in cancer patients with progressive cachexia. Clin Cancer Res, 13(21), 6379-6385. https://doi.org/10.1158/1078-0432.CCR-07-1147

Frame, S., Cohen, P., \& Biondi, R. M. (2001). A common phosphate binding site explains the unique substrate specificity of GSK3 and its inactivation by phosphorylation. Mol Cell, 7(6), 1321-1327. https://doi.org/10.1016/s10972765(01)00253-2

Fritzen, A. M., Lundsgaard, A. M., Jeppesen, J., Christiansen, M. L., Bienso, R., Dyck, J. R., ... Kiens, B. (2015). 5'-AMP activated protein kinase alpha2 controls substrate metabolism during post-exercise recovery via regulation of pyruvate dehydrogenase kinase 4. J Physiol, 593(21), 4765-4780. https://doi.org/10.1113/JP270821

Frost, R. A., \& Lang, C. H. (2007). Protein kinase B/Akt: a nexus of growth factor and cytokine signaling in determining muscle mass. J Appl Physiol (1985), 103(1), 378-387. https://doi.org/10.1152/japplphysiol.00089.2007

Fuchsberger, C., Flannick, J., Teslovich, T. M., Mahajan, A., Agarwala, V., Gaulton, K. J., . . McCarthy, M. I. (2016). The genetic architecture of type 2 diabetes. Nature, 536(7614), 41-47. https://doi.org/10.1038/nature18642

Fujii, N., Ho, R. C., Manabe, Y., Jessen, N., Toyoda, T., Holland, W. L., . . . Goodyear, L. J. (2008). Ablation of AMP-activated protein kinase alpha2 activity exacerbates insulin resistance induced by high-fat feeding of mice. Diabetes, 57(11), 2958-2966. https://doi.org/10.2337/db07-1187

Gamboa, J. L., Billings, F. T. t., Bojanowski, M. T., Gilliam, L. A., Yu, C., Roshanravan, B., . . . Brown, N. J. (2016). Mitochondrial dysfunction and oxidative stress in patients with chronic kidney disease. Physiol Rep, 4(9). https://doi.org/10.14814/phy2.12780

Gao, S., \& Carson, J. A. (2016). Lewis lung carcinoma regulation of mechanical stretchinduced protein synthesis in cultured myotubes. Am J Physiol Cell Physiol, 310(1), C66-79. https://doi.org/10.1152/ajpcell.00052.2015

Garnier, A., Fortin, D., Zoll, J., N'Guessan, B., Mettauer, B., Lampert, E., . . . VenturaClapier, R. (2005). Coordinated changes in mitochondrial function and biogenesis in healthy and diseased human skeletal muscle. FASEB J, 19(1), 43-52. https://doi.org/10.1096/fj.04-2173com 
Gerovasili, V., Stefanidis, K., Vitzilaios, K., Karatzanos, E., Politis, P., Koroneos, A., . . . Nanas, S. (2009). Electrical muscle stimulation preserves the muscle mass of critically ill patients: a randomized study. Crit Care, 13(5), R161. https://doi.org/10.1186/cc8123

Goodman, C. A., Mabrey, D. M., Frey, J. W., Miu, M. H., Schmidt, E. K., Pierre, P., \& Hornberger, T. A. (2011). Novel insights into the regulation of skeletal muscle protein synthesis as revealed by a new nonradioactive in vivo technique. FASEB $J, 25(3), 1028-1039$. https://doi.org/10.1096/fj.10-168799

Goodpaster, B. H., \& Sparks, L. M. (2017). Metabolic Flexibility in Health and Disease. Cell Metab, 25(5), 1027-1036. https://doi.org/10.1016/j.cmet.2017.04.015

Gordon, B. S., Steiner, J. L., Lang, C. H., Jefferson, L. S., \& Kimball, S. R. (2014). Reduced REDD1 expression contributes to activation of mTORC1 following electrically induced muscle contraction. Am J Physiol Endocrinol Metab, 307(8), E703-711. https://doi.org/10.1152/ajpendo.00250.2014

Gordon, B. S., Williamson, D. L., Lang, C. H., Jefferson, L. S., \& Kimball, S. R. (2015). Nutrient-induced stimulation of protein synthesis in mouse skeletal muscle is limited by the mTORC1 repressor REDD1. J Nutr, 145(4), 708-713. https://doi.org/10.3945/jn.114.207621

Gosker, H. R., van Mameren, H., van Dijk, P. J., Engelen, M. P., van der Vusse, G. J., Wouters, E. F., \& Schols, A. M. (2002). Skeletal muscle fibre-type shifting and metabolic profile in patients with chronic obstructive pulmonary disease. Eur Respir J, 19(4), 617-625. https://doi.org/10.1183/09031936.02.00762001

Graber, T. G., Fry, C. S., Brightwell, C. R., Moro, T., Maroto, R., Bhattarai, N., . . . Rasmussen, B. B. (2019). Skeletal muscle-specific knockout of DEP domain containing 5 protein increases mTORC1 signaling, muscle cell hypertrophy, and mitochondrial respiration. J Biol Chem, 294(11), 4091-4102. https://doi.org/10.1074/jbc.RA118.005970

Greer, E. L., Oskoui, P. R., Banko, M. R., Maniar, J. M., Gygi, M. P., Gygi, S. P., \& Brunet, A. (2007). The energy sensor AMP-activated protein kinase directly regulates the mammalian FOXO3 transcription factor. J Biol Chem, 282(41), 30107-30119. https://doi.org/10.1074/jbc.M705325200

Greising, S. M., Ottenheijm, C. A. C., O'Halloran, K. D., \& Barreiro, E. (2018). Diaphragm plasticity in aging and disease: therapies for muscle weakness go from strength to strength. J Appl Physiol (1985), 125(2), 243-253. https://doi.org/10.1152/japplphysiol.01059.2017

Gudiksen, A., Bertholdt, L., Vingborg, M. B., Hansen, H. W., Ringholm, S., \& Pilegaard, H. (2017). Muscle interleukin-6 and fasting-induced PDH regulation in mouse skeletal muscle. Am J Physiol Endocrinol Metab, 312(3), E204-E214. https://doi.org/10.1152/ajpendo.00291.2016

Guillory, B., Splenser, A., \& Garcia, J. (2013). The role of ghrelin in anorexia-cachexia syndromes. Vitam Horm, 92, 61-106. https://doi.org/10.1016/B978-0-12-410473$\underline{0.00003-9}$

Gwinn, D. M., Shackelford, D. B., Egan, D. F., Mihaylova, M. M., Mery, A., Vasquez, D. S., . . Shaw, R. J. (2008). AMPK phosphorylation of raptor mediates a metabolic checkpoint. Mol Cell, 30(2), 214-226. https://doi.org/10.1016/j.molcel.2008.03.003 
Hall, D. T., Griss, T., Ma, J. F., Sanchez, B. J., Sadek, J., Tremblay, A. M. K., . . . Gallouzi, I. E. (2018). The AMPK agonist 5-aminoimidazole-4-carboxamide ribonucleotide (AICAR), but not metformin, prevents inflammation-associated cachectic muscle wasting. EMBO Mol Med, 10(7). https://doi.org/10.15252/emmm.201708307

Hamada, T., Sasaki, H., Hayashi, T., Moritani, T., \& Nakao, K. (2003). Enhancement of whole body glucose uptake during and after human skeletal muscle low-frequency electrical stimulation. J Appl Physiol (1985), 94(6), 2107-2112. https://doi.org/10.1152/japplphysiol.00486.2002

Han, X., Raun, S. H., Carlsson, M., Sjoberg, K. A., Henriquez-Olguin, C., Ali, M., .. . Sylow, L. (2020). Cancer causes metabolic perturbations associated with reduced insulin-stimulated glucose uptake in peripheral tissues and impaired muscle microvascular perfusion. Metabolism, 105, 154169. https://doi.org/10.1016/j.metabol.2020.154169

Handberg, A., Vaag, A., Damsbo, P., Beck-Nielsen, H., \& Vinten, J. (1990). Expression of insulin regulatable glucose transporters in skeletal muscle from type 2 (noninsulin-dependent) diabetic patients. Diabetologia, 33(10), 625-627. https://doi.org/10.1007/BF00400207

Hanson, E. D., Nelson, A. R., West, D. W., Violet, J. A., O'Keefe, L., Phillips, S. M., \& Hayes, A. (2017). Attenuation of Resting but Not Load-Mediated Protein Synthesis in Prostate Cancer Patients on Androgen Deprivation. J Clin Endocrinol Metab, 102(3), 1076-1083. https://doi.org/10.1210/jc.2016-3383

Hardee, J. P., Counts, B. R., \& Carson, J. A. (2019). Understanding the Role of Exercise in Cancer Cachexia Therapy. Am J Lifestyle Med, 13(1), 46-60. https://doi.org/10.1177/1559827617725283

Hardee, J. P., Counts, B. R., Gao, S., VanderVeen, B. N., Fix, D. K., Koh, H. J., \& Carson, J. A. (2018). Inflammatory signalling regulates eccentric contractioninduced protein synthesis in cachectic skeletal muscle. J Cachexia Sarcopenia Muscle, 9(2), 369-383. https://doi.org/10.1002/jcsm.12271

Hardee, J. P., Fix, D. K., Koh, H. J., Wang, X., Goldsmith, E. C., \& Carson, J. A. (2020). Repeated eccentric contractions positively regulate muscle oxidative metabolism and protein synthesis during cancer cachexia in mice. J Appl Physiol (1985), 128(6), 1666-1676. https://doi.org/10.1152/japplphysiol.00908.2019

Hardee, J. P., Fix, D. K., Wang, X., Goldsmith, E. C., Koh, H. J., \& Carson, J. A. (2018). Systemic IL-6 regulation of eccentric contraction-induced muscle protein synthesis. Am J Physiol Cell Physiol, 315(1), C91-C103. https://doi.org/10.1152/ajpcell.00063.2018

Hardee, J. P., Mangum, J. E., Gao, S., Sato, S., Hetzler, K. L., Puppa, M. J., . . Carson, J. A. (2016). Eccentric contraction-induced myofiber growth in tumor-bearing mice. J Appl Physiol (1985), 120(1), 29-37. https://doi.org/10.1152/japplphysiol.00416.2015

Hardee, J. P., Montalvo, R. N., \& Carson, J. A. (2017). Linking Cancer CachexiaInduced Anabolic Resistance to Skeletal Muscle Oxidative Metabolism. Oxid Med Cell Longev, 2017, 8018197. https://doi.org/10.1155/2017/8018197 
Hardee, J. P., Puppa, M. J., Fix, D. K., Gao, S., Hetzler, K. L., Bateman, T. A., \& Carson, J. A. (2014). The effect of radiation dose on mouse skeletal muscle remodeling. Radiol Oncol, 48(3), 247-256. https://doi.org/10.2478/raon-2014-0025

Hardie, D. G. (2003). Minireview: the AMP-activated protein kinase cascade: the key sensor of cellular energy status. Endocrinology, 144(12), 5179-5183. https://doi.org/10.1210/en.2003-0982

Hardie, D. G., \& Pan, D. A. (2002). Regulation of fatty acid synthesis and oxidation by the AMP-activated protein kinase. Biochem Soc Trans, 30(Pt 6), 1064-1070. https://doi.org/10.1042/bst0301064

Harfmann, B. D., Schroder, E. A., \& Esser, K. A. (2015). Circadian rhythms, the molecular clock, and skeletal muscle. J Biol Rhythms, 30(2), 84-94. https://doi.org/10.1177/0748730414561638

Harfmann, B. D., Schroder, E. A., Kachman, M. T., Hodge, B. A., Zhang, X., \& Esser, K. A. (2016). Muscle-specific loss of Bmall leads to disrupted tissue glucose metabolism and systemic glucose homeostasis. Skelet Muscle, 6, 12. https://doi.org/10.1186/s13395-016-0082-X

Harridge, S. D., Andersen, J. L., Hartkopp, A., Zhou, S., Biering-Sorensen, F., Sandri, C., \& Kjaer, M. (2002). Training by low-frequency stimulation of tibialis anterior in spinal cord-injured men. Muscle Nerve, 25(5), 685-694. https://doi.org/10.1002/mus.10021

Hatting, M., Spannbauer, M., Peng, J., Al Masaoudi, M., Sellge, G., Nevzorova, Y. A., . . . Trautwein, C. (2015). Lack of gp130 expression in hepatocytes attenuates tumor progression in the DEN model. Cell Death Dis, 6, e1667. https://doi.org/10.1038/cddis.2014.590

Hawley, J. A. (2004). Exercise as a therapeutic intervention for the prevention and treatment of insulin resistance. Diabetes Metab Res Rev, 20(5), 383-393. https://doi.org/10.1002/dmrr.505

Hawley, J. A., \& Lessard, S. J. (2008). Exercise training-induced improvements in insulin action. Acta Physiol (Oxf), 192(1), 127-135. https://doi.org/10.1111/j.17481716.2007.01783.x

Herzig, S., \& Shaw, R. J. (2018). AMPK: guardian of metabolism and mitochondrial homeostasis. Nat Rev Mol Cell Biol, 19(2), 121-135. https://doi.org/10.1038/nrm.2017.95

Hingst, J. R., Kjobsted, R., Birk, J. B., Jorgensen, N. O., Larsen, M. R., Kido, K., . . . Wojtaszewski, J. F. P. (2020). Inducible deletion of skeletal muscle AMPKalpha reveals that AMPK is required for nucleotide balance but dispensable for muscle glucose uptake and fat oxidation during exercise. Mol Metab, 40, 101028. https://doi.org/10.1016/j.molmet.2020.101028

Hirota, H., Chen, J., Betz, U. A., Rajewsky, K., Gu, Y., Ross, J., Jr., . . Chien, K. R. (1999). Loss of a gp130 cardiac muscle cell survival pathway is a critical event in the onset of heart failure during biomechanical stress. Cell, 97(2), 189-198. https://doi.org/10.1016/s0092-8674(00)80729-1

Ho, A., Cho, C. S., Namkoong, S., Cho, U. S., \& Lee, J. H. (2016). Biochemical Basis of Sestrin Physiological Activities. Trends Biochem Sci, 41(7), 621-632. https://doi.org/10.1016/j.tibs.2016.04.005 
Hodson, N., McGlory, C., Oikawa, S. Y., Jeromson, S., Song, Z., Ruegg, M. A., . . Philp, A. (2017). Differential localization and anabolic responsiveness of mTOR complexes in human skeletal muscle in response to feeding and exercise. Am J Physiol Cell Physiol, 313(6), C604-C611. https://doi.org/10.1152/ajpcell.00176.2017

Hody, S., Croisier, J. L., Bury, T., Rogister, B., \& Leprince, P. (2019). Eccentric Muscle Contractions: Risks and Benefits. Front Physiol, 10, 536. https://doi.org/10.3389/fphys.2019.00536

Holloszy, J. O., \& Coyle, E. F. (1984). Adaptations of skeletal muscle to endurance exercise and their metabolic consequences. J Appl Physiol Respir Environ Exerc Physiol, 56(4), 831-838. https://doi.org/10.1152/jappl.1984.56.4.831

Hood, D. A., Irrcher, I., Ljubicic, V., \& Joseph, A. M. (2006). Coordination of metabolic plasticity in skeletal muscle. J Exp Biol, 209(Pt 12), 2265-2275. https://doi.org/10.1242/jeb.02182

Hornberger, T. A. (2011). Mechanotransduction and the regulation of mTORC1 signaling in skeletal muscle. Int J Biochem Cell Biol, 43(9), 1267-1276. https://doi.org/10.1016/j.biocel.2011.05.007

Horstman, A. M., Olde Damink, S. W., Schols, A. M., \& van Loon, L. J. (2016). Is Cancer Cachexia Attributed to Impairments in Basal or Postprandial Muscle Protein Metabolism? Nutrients, 8(8). https://doi.org/10.3390/nu8080499

Hua, N., Takahashi, H., Yee, G. M., Kitajima, Y., Katagiri, S., Kojima, M., . . . Hamilton, J. A. (2017). Influence of muscle fiber type composition on early fat accumulation under high-fat diet challenge. PLoS One, 12(8), e0182430. https://doi.org/10.1371/journal.pone.0182430

Huang, J., \& Zhu, X. (2016). The molecular mechanisms of calpains action on skeletal muscle atrophy. Physiol Res, 65(4), 547-560. https://doi.org/10.33549/physiolres.933087

Iglewski, M., Hill, J. A., Lavandero, S., \& Rothermel, B. A. (2010). Mitochondrial fission and autophagy in the normal and diseased heart. Curr Hypertens Rep, 12(6), 418-425. https://doi.org/10.1007/s11906-010-0147-x

Jafri, S. H., Previgliano, C., Khandelwal, K., \& Shi, R. (2015). Cachexia Index in Advanced Non-Small-Cell Lung Cancer Patients. Clin Med Insights Oncol, 9, 8793. https://doi.org/10.4137/CMO.S30891

Jager, S., Handschin, C., St-Pierre, J., \& Spiegelman, B. M. (2007). AMP-activated protein kinase (AMPK) action in skeletal muscle via direct phosphorylation of PGC-1alpha. Proc Natl Acad Sci U S A, 104(29), 12017-12022. https://doi.org/10.1073/pnas.0705070104

Jatoi, A., Daly, B. D., Hughes, V., Dallal, G. E., \& Roubenoff, R. (1999). The prognostic effect of increased resting energy expenditure prior to treatment for lung cancer. Lung Cancer, 23(2), 153-158. https://doi.org/10.1016/s0169-5002(99)00008-2

Jebb, S. A., Osborne, R. J., Dixon, A. K., Bleehen, N. M., \& Elia, M. (1994). Measurements of resting energy expenditure and body composition before and after treatment of small cell lung cancer. Ann Oncol, 5(10), 915-919. https://doi.org/10.1093/oxfordjournals.annonc.a058730 
Jee, H., Chang, J. E., \& Yang, E. J. (2016). Positive Prehabilitative Effect of Intense Treadmill Exercise for Ameliorating Cancer Cachexia Symptoms in a Mouse Model. J Cancer, 7(15), 2378-2387. https://doi.org/10.7150/jca.17162

Jensen, M. M., Jorgensen, J. T., Binderup, T., \& Kjaer, A. (2008). Tumor volume in subcutaneous mouse xenografts measured by microCT is more accurate and reproducible than determined by $18 \mathrm{~F}-\mathrm{FDG}$-microPET or external caliper. $B M C$ Med Imaging, 8, 16. https://doi.org/10.1186/1471-2342-8-16

Jensen, T. L., Kiersgaard, M. K., Sorensen, D. B., \& Mikkelsen, L. F. (2013). Fasting of mice: a review. Lab Anim, 47(4), 225-240. https://doi.org/10.1177/0023677213501659

Jeon, S. M. (2016). Regulation and function of AMPK in physiology and diseases. Exp Mol Med, 48(7), e245. https://doi.org/10.1038/emm.2016.81

Johns, N., Hatakeyama, S., Stephens, N. A., Degen, M., Degen, S., Frieauff, W., . . . Fearon, K. C. (2014). Clinical classification of cancer cachexia: phenotypic correlates in human skeletal muscle. PLoS One, 9(1), e83618. https://doi.org/10.1371/journal.pone.0083618

Jorgensen, S. B., Treebak, J. T., Viollet, B., Schjerling, P., Vaulont, S., Wojtaszewski, J. F., \& Richter, E. A. (2007). Role of AMPKalpha2 in basal, training-, and AICARinduced GLUT4, hexokinase II, and mitochondrial protein expression in mouse muscle. Am J Physiol Endocrinol Metab, 292(1), E331-339. https://doi.org/10.1152/ajpendo.00243.2006

Jornayvaz, F. R., \& Shulman, G. I. (2010). Regulation of mitochondrial biogenesis. Essays Biochem, 47, 69-84. https://doi.org/10.1042/bse0470069

Julienne, C. M., Dumas, J. F., Goupille, C., Pinault, M., Berri, C., Collin, A., . . Servais, S. (2012). Cancer cachexia is associated with a decrease in skeletal muscle mitochondrial oxidative capacities without alteration of ATP production efficiency. J Cachexia Sarcopenia Muscle, 3(4), 265-275. https://doi.org/10.1007/s13539-012-0071-9

Kang, M. J., Moon, J. W., Lee, J. O., Kim, J. H., Jung, E. J., Kim, S. J., . . Kim, H. S. (2021). Metformin induces muscle atrophy by transcriptional regulation of myostatin via HDAC6 and FoxO3a. J Cachexia Sarcopenia Muscle. https://doi.org/10.1002/jcsm. 12833

Kellar, A., Egan, C., \& Morris, D. (2015). Preclinical Murine Models for Lung Cancer: Clinical Trial Applications. Biomed Res Int, 2015, 621324. https://doi.org/10.1155/2015/621324

Kessels, E., Husson, O., \& van der Feltz-Cornelis, C. M. (2018). The effect of exercise on cancer-related fatigue in cancer survivors: a systematic review and metaanalysis. Neuropsychiatr Dis Treat, 14, 479-494. https://doi.org/10.2147/NDT.S150464

Khodabukus, A., \& Baar, K. (2015). Contractile and metabolic properties of engineered skeletal muscle derived from slow and fast phenotype mouse muscle. J Cell Physiol, 230(8), 1750-1757. https://doi.org/10.1002/jcp.24848

Kim, J., Yang, G., Kim, Y., Kim, J., \& Ha, J. (2016). AMPK activators: mechanisms of action and physiological activities. Exp Mol Med, 48, e224. https://doi.org/10.1038/emm.2016.16 
Kimball, S. R., \& Jefferson, L. S. (2010). Control of translation initiation through integration of signals generated by hormones, nutrients, and exercise. J Biol Chem, 285(38), 29027-29032. https://doi.org/10.1074/jbc.R110.137208

Kimball, S. R., Shantz, L. M., Horetsky, R. L., \& Jefferson, L. S. (1999). Leucine regulates translation of specific mRNAs in L6 myoblasts through mTORmediated changes in availability of eIF4E and phosphorylation of ribosomal protein S6. J Biol Chem, 274(17), 11647-11652. https://doi.org/10.1074/jbc.274.17.11647

Kishimoto, T., Taga, T., \& Akira, S. (1994). Cytokine signal transduction. Cell, 76(2), 253-262. https://doi.org/10.1016/0092-8674(94)90333-6

Kjobsted, R., Hingst, J. R., Fentz, J., Foretz, M., Sanz, M. N., Pehmoller, C., . . L Lantier, L. (2018). AMPK in skeletal muscle function and metabolism. FASEB J, 32(4), 1741-1777. https://doi.org/10.1096/fj.201700442R

Klionsky, D. J. (2005). The molecular machinery of autophagy: unanswered questions. $J$ Cell Sci, 118(Pt 1), 7-18. https://doi.org/10.1242/jcs.01620

Klok, M. D., Jakobsdottir, S., \& Drent, M. L. (2007). The role of leptin and ghrelin in the regulation of food intake and body weight in humans: a review. Obes Rev, 8(1), 21-34. https://doi.org/10.1111/j.1467-789X.2006.00270.x

Koh, H. J., Brandauer, J., \& Goodyear, L. J. (2008). LKB1 and AMPK and the regulation of skeletal muscle metabolism. Curr Opin Clin Nutr Metab Care, 11(3), 227-232. https://doi.org/10.1097/MCO.0b013e3282fb7b76

Koves, T. R., Sparks, L. M., Kovalik, J. P., Mosedale, M., Arumugam, R., DeBalsi, K. L., . . Muoio, D. M. (2013). PPARgamma coactivator-1 alpha contributes to exercise-induced regulation of intramuscular lipid droplet programming in mice and humans. J Lipid Res, 54(2), 522-534. https://doi.org/10.1194/jlr.P028910

Kunzke, T., Buck, A., Prade, V. M., Feuchtinger, A., Prokopchuk, O., Martignoni, M. E., ... Aichler, M. (2020). Derangements of amino acids in cachectic skeletal muscle are caused by mitochondrial dysfunction. J Cachexia Sarcopenia Muscle, 11(1), 226-240. https://doi.org/10.1002/jcsm. 12498

Laker, R. C., Drake, J. C., Wilson, R. J., Lira, V. A., Lewellen, B. M., Ryall, K. A., .. . Yan, Z. (2017). Ampk phosphorylation of Ulk1 is required for targeting of mitochondria to lysosomes in exercise-induced mitophagy. Nat Commun, 8(1), 548. https://doi.org/10.1038/s41467-017-00520-9

Lam, Y. Y., \& Ravussin, E. (2016). Analysis of energy metabolism in humans: A review of methodologies. Mol Metab, 5(11), 1057-1071.

https://doi.org/10.1016/j.molmet.2016.09.005

Lammers, L. A., Achterbergh, R., Mathot, R. A. A., \& Romijn, J. A. (2020). The effects of fasting on drug metabolism. Expert Opin Drug Metab Toxicol, 16(1), 79-85. https://doi.org/10.1080/17425255.2020.1706728

Lantier, L., Williams, A. S., Williams, I. M., Guerin, A., Bracy, D. P., Goelzer, M., .. . Wasserman, D. H. (2020). Reciprocity Between Skeletal Muscle AMPK Deletion and Insulin Action in Diet-Induced Obese Mice. Diabetes, 69(8), 1636-1649. https://doi.org/10.2337/db19-1074

Lanza, I. R., \& Nair, K. S. (2009). Functional assessment of isolated mitochondria in vitro. Methods Enzymol, 457, 349-372. https://doi.org/10.1016/S0076$\underline{6879(09) 05020-4}$ 
Laplante, M., \& Sabatini, D. M. (2009). mTOR signaling at a glance. J Cell Sci, 122(Pt 20), 3589-3594. https://doi.org/10.1242/jcs.051011

Lecker, S. H., Solomon, V., Mitch, W. E., \& Goldberg, A. L. (1999). Muscle protein breakdown and the critical role of the ubiquitin-proteasome pathway in normal and disease states. J Nutr, 129(1S Suppl), 227S-237S. https://doi.org/10.1093/jn/129.1.227S

Lee, H. W., Baker, E., Lee, K. M., Persinger, A. M., Hawkins, W., \& Puppa, M. (2019). Effects of low-dose leucine supplementation on gastrocnemius muscle mitochondrial content and protein turnover in tumor-bearing mice. Appl Physiol Nutr Metab, 44(9), 997-1004. https://doi.org/10.1139/apnm-2018-0765

Levine, B., \& Kroemer, G. (2008). Autophagy in the pathogenesis of disease. Cell, 132(1), 27-42. https://doi.org/10.1016/i.cell.2007.12.018

Lira, V. A., Okutsu, M., Zhang, M., Greene, N. P., Laker, R. C., Breen, D. S., . . Yan, Z. (2013). Autophagy is required for exercise training-induced skeletal muscle adaptation and improvement of physical performance. Faseb Journal, 27(10), 4184-4193. https://doi.org/10.1096/fj.13-228486

Liu, G. Y., \& Sabatini, D. M. (2020). Author Correction: mTOR at the nexus of nutrition, growth, ageing and disease. Nat Rev Mol Cell Biol, 21(4), 246. https://doi.org/10.1038/s41580-020-0219-y

Liu, H., Luo, J., Guillory, B., Chen, J. A., Zang, P., Yoeli, J. K., . . Garcia, J. M. (2020). Ghrelin ameliorates tumor-induced adipose tissue atrophy and inflammation via Ghrelin receptor-dependent and -independent pathways. Oncotarget, 11(35), 3286-3302. https://doi.org/10.18632/oncotarget.27705

Lombardi, G., Zustovich, F., Farina, P., Polo, V., Farina, M., Della Puppa, A., . . . Zagonel, V. (2012). Cilengitide in bevacizumab-refractory high-grade glioma: two case reports and critical review of the literature. Anticancer Drugs, 23(7), 749-753. https://doi.org/10.1097/CAD.0b013e3283520e2c

Long, Y. C., \& Zierath, J. R. (2006). AMP-activated protein kinase signaling in metabolic regulation. J Clin Invest, 116(7), 1776-1783. https://doi.org/10.1172/JCI29044

Lorite, M. J., Thompson, M. G., Drake, J. L., Carling, G., \& Tisdale, M. J. (1998). Mechanism of muscle protein degradation induced by a cancer cachectic factor. Br J Cancer, 78(7), 850-856. https://doi.org/10.1038/bjc.1998.592

Luo, J., Sobkiw, C. L., Hirshman, M. F., Logsdon, M. N., Li, T. Q., Goodyear, L. J., \& Cantley, L. C. (2006). Loss of class IA PI3K signaling in muscle leads to impaired muscle growth, insulin response, and hyperlipidemia. Cell Metab, 3(5), 355-366. https://doi.org/10.1016/j.cmet.2006.04.003

Ma, D., Wang, Y., Zhou, G., Wang, Y., \& Li, X. (2019). Review: the Roles and Mechanisms of Glycoprotein 130 Cytokines in the Regulation of Adipocyte Biological Function. Inflammation, 42(3), 790-798. https://doi.org/10.1007/s10753-019-00959-6

Maarbjerg, S. J., Jorgensen, S. B., Rose, A. J., Jeppesen, J., Jensen, T. E., Treebak, J. T., . . . Richter, E. A. (2009). Genetic impairment of AMPKalpha2 signaling does not reduce muscle glucose uptake during treadmill exercise in mice. Am J Physiol Endocrinol Metab, 297(4), E924-934. https://doi.org/10.1152/ajpendo.90653.2008 
Maddocks, M., Murton, A. J., \& Wilcock, A. (2011). Improving muscle mass and function in cachexia: non-drug approaches. Curr Opin Support Palliat Care, 5(4), 361-364. https://doi.org/10.1097/SPC.0b013e32834bdde3

Marimuthu, K., Murton, A. J., \& Greenhaff, P. L. (2011). Mechanisms regulating muscle mass during disuse atrophy and rehabilitation in humans. J Appl Physiol (1985), 110(2), 555-560. https://doi.org/10.1152/japplphysiol.00962.2010

Martina, J. A., Chen, Y., Gucek, M., \& Puertollano, R. (2012). MTORC1 functions as a transcriptional regulator of autophagy by preventing nuclear transport of TFEB. Autophagy, 8(6), 903-914. https://doi.org/10.4161/auto.19653

Marzetti, E., Lorenzi, M., Landi, F., Picca, A., Rosa, F., Tanganelli, F., . . Bossola, M. (2017). Altered mitochondrial quality control signaling in muscle of old gastric cancer patients with cachexia. Exp Gerontol, 87(Pt A), 92-99. https://doi.org/10.1016/j.exger.2016.10.003

McCarthy, J. J., Srikuea, R., Kirby, T. J., Peterson, C. A., \& Esser, K. A. (2012). Inducible Cre transgenic mouse strain for skeletal muscle-specific gene targeting. Skelet Muscle, 2(1), 8. https://doi.org/10.1186/2044-5040-2-8

Mehl, K. A., Davis, J. M., Clements, J. M., Berger, F. G., Pena, M. M., \& Carson, J. A. (2005). Decreased intestinal polyp multiplicity is related to exercise mode and gender in ApcMin/+ mice. J Appl Physiol (1985), 98(6), 2219-2225. https://doi.org/10.1152/japplphysiol.00975.2004

Michaelis, K. A., Zhu, X., Burfeind, K. G., Krasnow, S. M., Levasseur, P. R., Morgan, T. K., \& Marks, D. L. (2017). Establishment and characterization of a novel murine model of pancreatic cancer cachexia. J Cachexia Sarcopenia Muscle, 8(5), 824838. https://doi.org/10.1002/jcsm.12225

Miinea, C. P., Sano, H., Kane, S., Sano, E., Fukuda, M., Peranen, J., . . Lienhard, G. E. (2005). AS160, the Akt substrate regulating GLUT4 translocation, has a functional Rab GTPase-activating protein domain. Biochem J, 391(Pt 1), 87-93. https://doi.org/10.1042/BJ20050887

Miura, S., Kai, Y., Kamei, Y., Bruce, C. R., Kubota, N., Febbraio, M. A., . . Ezaki, O. (2009). Alpha2-AMPK activity is not essential for an increase in fatty acid oxidation during low-intensity exercise. Am J Physiol Endocrinol Metab, 296(1), E47-55. https://doi.org/10.1152/ajpendo.90690.2008

Moller, L. L., Sylow, L., Gotzsche, C. R., Serup, A. K., Christiansen, S. H., Weikop, P., . . . Richter, E. A. (2016). Decreased spontaneous activity in AMPK alpha2 muscle specific kinase dead mice is not caused by changes in brain dopamine metabolism. Physiol Behav, 164(Pt A), 300-305. https://doi.org/10.1016/j.physbeh.2016.06.010

Molyneaux, K. A., Schaible, K., \& Wylie, C. (2003). GP130, the shared receptor for the LIF/IL6 cytokine family in the mouse, is not required for early germ cell differentiation, but is required cell-autonomously in oocytes for ovulation. Development, 130(18), 4287-4294. https://doi.org/10.1242/dev.00650

Monitto, C. L., Berkowitz, D., Lee, K. M., Pin, S., Li, D., Breslow, M., . . . Schiller, M. (2001). Differential gene expression in a murine model of cancer cachexia. Am J Physiol Endocrinol Metab, 281(2), E289-297. https://doi.org/10.1152/ajpendo.2001.281.2.E289 
Moore, D. R. (2019). Maximizing Post-exercise Anabolism: The Case for Relative Protein Intakes. Front Nutr, 6, 147. https://doi.org/10.3389/fnut.2019.00147 Morissette, M. P., Susser, S. E., Stammers, A. N., O'Hara, K. A., Gardiner, P. F., Sheppard, P., . . Duhamel, T. A. (2014). Differential regulation of the fiber typespecific gene expression of the sarcoplasmic reticulum calcium-ATPase isoforms induced by exercise training. J Appl Physiol (1985), 117(5), 544-555.

https://doi.org/10.1152/japplphysiol.00092.2014

Morita, M., Prudent, J., Basu, K., Goyon, V., Katsumura, S., Hulea, L., . . . Sonenberg, N. (2017). mTOR Controls Mitochondrial Dynamics and Cell Survival via MTFP1. Mol Cell, 67(6), 922-935 e925. https://doi.org/10.1016/j.molcel.2017.08.013

Moro, T., Ebert, S. M., Adams, C. M., \& Rasmussen, B. B. (2016). Amino Acid Sensing in Skeletal Muscle. Trends Endocrinol Metab, 27(11), 796-806. https://doi.org/10.1016/j.tem.2016.06.010

Morris, V. A., Punjabi, A. S., \& Lagunoff, M. (2008). Activation of Akt through gp130 receptor signaling is required for Kaposi's sarcoma-associated herpesvirusinduced lymphatic reprogramming of endothelial cells. J Virol, 82(17), 87718779. https://doi.org/10.1128/JVI.00766-08

Morton, R. W., Traylor, D. A., Weijs, P. J. M., \& Phillips, S. M. (2018). Defining anabolic resistance: implications for delivery of clinical care nutrition. Curr Opin Crit Care, 24(2), 124-130. https://doi.org/10.1097/MCC.0000000000000488

Moser, A. R., Pitot, H. C., \& Dove, W. F. (1990). A dominant mutation that predisposes to multiple intestinal neoplasia in the mouse. Science, 247(4940), 322-324. https://doi.org/10.1126/science.2296722

Motoshima, H., Goldstein, B. J., Igata, M., \& Araki, E. (2006). AMPK and cell proliferation--AMPK as a therapeutic target for atherosclerosis and cancer. $J$ Physiol, 574(Pt 1), 63-71. https://doi.org/10.1113/jphysiol.2006.108324

Mulligan, J. D., Gonzalez, A. A., Kumar, R., Davis, A. J., \& Saupe, K. W. (2005). Aging elevates basal adenosine monophosphate-activated protein kinase (AMPK) activity and eliminates hypoxic activation of AMPK in mouse liver. $J$ Gerontol A Biol Sci Med Sci, 60(1), 21-27. https://doi.org/10.1093/gerona/60.1.21

Murphy, K. T., Chee, A., Trieu, J., Naim, T., \& Lynch, G. S. (2012). Importance of functional and metabolic impairments in the characterization of the C-26 murine model of cancer cachexia. Dis Model Mech, 5(4), 533-545. https://doi.org/10.1242/dmm.008839

Murphy, K. T., Hossain, M. I., Swiderski, K., Chee, A., Naim, T., Trieu, J., . . Lynch, G. S. (2019). Mas Receptor Activation Slows Tumor Growth and Attenuates Muscle Wasting in Cancer. Cancer Res, 79(4), 706-719. https://doi.org/10.1158/00085472.CAN-18-1207

Murton, A. J., Constantin, D., \& Greenhaff, P. L. (2008). The involvement of the ubiquitin proteasome system in human skeletal muscle remodelling and atrophy. Biochimica Et Biophysica Acta-Molecular Basis of Disease, 1782(12), 730-743. https://doi.org/10.1016/j.bbadis.2008.10.011

Muscaritoli, M., Anker, S. D., Argiles, J., Aversa, Z., Bauer, J. M., Biolo, G., . . Sieber, C. C. (2010). Consensus definition of sarcopenia, cachexia and pre-cachexia: joint document elaborated by Special Interest Groups (SIG) "cachexia-anorexia in 
chronic wasting diseases" and "nutrition in geriatrics". Clin Nutr, 29(2), 154-159. https://doi.org/10.1016/j.clnu.2009.12.004

Myers, J., Kokkinos, P., \& Nyelin, E. (2019). Physical Activity, Cardiorespiratory Fitness, and the Metabolic Syndrome. Nutrients, 11(7). https://doi.org/10.3390/nu11071652

Nader, G. A., \& Esser, K. A. (2001). Intracellular signaling specificity in skeletal muscle in response to different modes of exercise. J Appl Physiol (1985), 90(5), 19361942. https://doi.org/10.1152/jappl.2001.90.5.1936

Nakayama, K., Tagawa, R., Saito, Y., \& Sanbongi, C. (2019). Effects of whey protein hydrolysate ingestion on post-exercise muscle protein synthesis compared with intact whey protein in rats. Nutr Metab (Lond), 16, 90. https://doi.org/10.1186/s12986-019-0417-9

Narsale, A. A., Puppa, M. J., Hardee, J. P., VanderVeen, B. N., Enos, R. T., Murphy, E. A., \& Carson, J. A. (2016). Short-term pyrrolidine dithiocarbamate administration attenuates cachexia-induced alterations to muscle and liver in ApcMin/+ mice. Oncotarget, 7(37), 59482-59502. https://doi.org/10.18632/oncotarget.10699

Nencioni, A., Caffa, I., Cortellino, S., \& Longo, V. D. (2018). Fasting and cancer: molecular mechanisms and clinical application. Nat Rev Cancer, 18(11), 707-719. https://doi.org/10.1038/s41568-018-0061-0

O'Neill, H. M. (2013). AMPK and Exercise: Glucose Uptake and Insulin Sensitivity. Diabetes Metab J, 37(1), 1-21. https://doi.org/10.4093/dmj.2013.37.1.1

O'Neill, H. M., Maarbjerg, S. J., Crane, J. D., Jeppesen, J., Jorgensen, S. B., Schertzer, J. D., . . Steinberg, G. R. (2011). AMP-activated protein kinase (AMPK) beta1beta2 muscle null mice reveal an essential role for AMPK in maintaining mitochondrial content and glucose uptake during exercise. Proc Natl Acad Sci U $S$ A, 108(38), 16092-16097. https://doi.org/10.1073/pnas.1105062108

Ohtsuki, I., \& Morimoto, S. (2008). Troponin: regulatory function and disorders. Biochem Biophys Res Commun, 369(1), 62-73. https://doi.org/10.1016/j.bbrc.2007.11.187

Oliveira, A. G., \& Gomes-Marcondes, M. C. (2016). Metformin treatment modulates the tumour-induced wasting effects in muscle protein metabolism minimising the cachexia in tumour-bearing rats. BMC Cancer, 16, 418. https://doi.org/10.1186/s12885-016-2424-9

Ost, M., Coleman, V., Kasch, J., \& Klaus, S. (2016). Regulation of myokine expression: Role of exercise and cellular stress. Free Radic Biol Med, 98, 78-89. https://doi.org/10.1016/j.freeradbiomed.2016.02.018

Paulussen, K. J. M., McKenna, C. F., Beals, J. W., Wilund, K. R., Salvador, A. F., \& Burd, N. A. (2021). Anabolic Resistance of Muscle Protein Turnover Comes in Various Shapes and Sizes. Front Nutr, 8, 615849. https://doi.org/10.3389/fnut.2021.615849

Penna, F., Ballaro, R., Beltra, M., De Lucia, S., Garcia Castillo, L., \& Costelli, P. (2019). The Skeletal Muscle as an Active Player Against Cancer Cachexia. Front Physiol, 10, 41. https://doi.org/10.3389/fphys.2019.00041

Penna, F., Ballaro, R., Martinez-Cristobal, P., Sala, D., Sebastian, D., Busquets, S., . . . Zorzano, A. (2019). Autophagy Exacerbates Muscle Wasting in Cancer Cachexia 
and Impairs Mitochondrial Function. J Mol Biol, 431(15), 2674-2686.

https://doi.org/10.1016/j.jmb.2019.05.032

Peronnet, F., \& Massicotte, D. (1991). Table of nonprotein respiratory quotient: an update. Can J Sport Sci, 16(1), 23-29.

https://www.ncbi.nlm.nih.gov/pubmed/1645211

Pettersen, K., Andersen, S., Degen, S., Tadini, V., Grosjean, J., Hatakeyama, S., .. . Bjorkoy, G. (2017). Cancer cachexia associates with a systemic autophagyinducing activity mimicked by cancer cell-derived IL-6 trans-signaling. Sci Rep, 7(1), 2046. https://doi.org/10.1038/s41598-017-02088-2

Phillips, S. M. (2014). A brief review of critical processes in exercise-induced muscular hypertrophy. Sports Med, 44 Suppl 1, S71-77. https://doi.org/10.1007/s40279014-0152-3

Picca, A., Lezza, A. M. S., Leeuwenburgh, C., Pesce, V., Calvani, R., Landi, F., . . . Marzetti, E. (2017). Fueling Inflamm-Aging through Mitochondrial Dysfunction: Mechanisms and Molecular Targets. Int J Mol Sci, 18(5). https://doi.org/10.3390/ijms18050933

Pigna, E., Berardi, E., Aulino, P., Rizzuto, E., Zampieri, S., Carraro, U., . . Moresi, V. (2016). Aerobic Exercise and Pharmacological Treatments Counteract Cachexia by Modulating Autophagy in Colon Cancer. Sci Rep, 6, 26991. https://doi.org/10.1038/srep26991

Pilegaard, H., Saltin, B., \& Neufer, P. D. (2003). Exercise induces transient transcriptional activation of the PGC-1alpha gene in human skeletal muscle. $J$ Physiol, 546(Pt 3), 851-858. https://doi.org/10.1113/jphysiol.2002.034850

Pin, F., Barreto, R., Kitase, Y., Mitra, S., Erne, C. E., Novinger, L. J., . . Bonetto, A. (2018). Growth of ovarian cancer xenografts causes loss of muscle and bone mass: a new model for the study of cancer cachexia. J Cachexia Sarcopenia Muscle, 9(4), 685-700. https://doi.org/10.1002/jcsm.12311

Porporato, P. E., Filigheddu, N., Reano, S., Ferrara, M., Angelino, E., Gnocchi, V. F., . . . Graziani, A. (2013). Acylated and unacylated ghrelin impair skeletal muscle atrophy in mice. J Clin Invest, 123(2), 611-622. https://doi.org/10.1172/JCI39920

Puppa, M. J., Gao, S., Narsale, A. A., \& Carson, J. A. (2014). Skeletal muscle glycoprotein 130's role in Lewis lung carcinoma-induced cachexia. FASEB $J$, 28(2), 998-1009. https://doi.org/10.1096/fj.13-240580

Puppa, M. J., Murphy, E. A., Fayad, R., Hand, G. A., \& Carson, J. A. (2014). Cachectic skeletal muscle response to a novel bout of low-frequency stimulation. $J \mathrm{Appl}$ Physiol (1985), 116(8), 1078-1087. https://doi.org/10.1152/japplphysiol.01270.2013

Quinn, J. M., Tam, S., Sims, N. A., Saleh, H., McGregor, N. E., Poulton, I. J., . . van Denderen, B. J. (2010). Germline deletion of AMP-activated protein kinase beta subunits reduces bone mass without altering osteoclast differentiation or function. FASEB J, 24(1), 275-285. https://doi.org/10.1096/fj.09-137158

Ravanan, P., Srikumar, I. F., \& Talwar, P. (2017). Autophagy: The spotlight for cellular stress responses. Life Sci, 188, 53-67. https://doi.org/10.1016/j.lfs.2017.08.029

Ravasco, P., Monteiro-Grillo, I., \& Camilo, M. E. (2003). Does nutrition influence quality of life in cancer patients undergoing radiotherapy? Radiother Oncol, 67(2), 213-220. https://doi.org/10.1016/s0167-8140(03)00040-9 
Re Cecconi, A. D., Forti, M., Chiappa, M., Zhu, Z., Zingman, L. V., Cervo, L., . . . Piccirillo, R. (2019). Musclin, A Myokine Induced by Aerobic Exercise, Retards Muscle Atrophy During Cancer Cachexia in Mice. Cancers (Basel), 11(10). https://doi.org/10.3390/cancers11101541

Richter, E. A., \& Hargreaves, M. (2013). Exercise, GLUT4, and skeletal muscle glucose uptake. Physiol Rev, 93(3), 993-1017.

https://doi.org/10.1152/physrev.00038.2012

Roberson, P. A., Shimkus, K. L., Welles, J. E., Xu, D., Whitsell, A. L., Kimball, E. M., . . . Kimball, S. R. (2020). A time course for markers of protein synthesis and degradation with hindlimb unloading and the accompanying anabolic resistance to refeeding. J Appl Physiol (1985), 129(1), 36-46.

https://doi.org/10.1152/japplphysiol.00155.2020

Roberts, B. M., Ahn, B., Smuder, A. J., Al-Rajhi, M., Gill, L. C., Beharry, A. W., . . Judge, A. R. (2013). Diaphragm and ventilatory dysfunction during cancer cachexia. FASEB J, 27(7), 2600-2610. https://doi.org/10.1096/fj.12-222844

Roberts, B. M., Frye, G. S., Ahn, B., Ferreira, L. F., \& Judge, A. R. (2013). Cancer cachexia decreases specific force and accelerates fatigue in limb muscle. Biochem Biophys Res Commun, 435(3), 488-492. https://doi.org/10.1016/j.bbrc.2013.05.018

Roeland, E. J., Bohlke, K., Baracos, V. E., Bruera, E., Del Fabbro, E., Dixon, S., . . Loprinzi, C. L. (2020). Management of Cancer Cachexia: ASCO Guideline. $J$ Clin Oncol, 38(21), 2438-2453. https://doi.org/10.1200/JCO.20.00611

Romanello, V., Guadagnin, E., Gomes, L., Roder, I., Sandri, C., Petersen, Y., . . Sandri, M. (2010). Mitochondrial fission and remodelling contributes to muscle atrophy. EMBO J, 29(10), 1774-1785. https://doi.org/10.1038/emboj.2010.60

Romanello, V., \& Sandri, M. (2010). Mitochondrial biogenesis and fragmentation as regulators of muscle protein degradation. Curr Hypertens Rep, 12(6), 433-439. https://doi.org/10.1007/s11906-010-0157-8

Romanello, V., \& Sandri, M. (2015). Mitochondrial Quality Control and Muscle Mass Maintenance. Front Physiol, 6, 422. https://doi.org/10.3389/fphys.2015.00422

Ross, P. J., Ashley, S., Norton, A., Priest, K., Waters, J. S., Eisen, T., . . O'Brien, M. E. (2004). Do patients with weight loss have a worse outcome when undergoing chemotherapy for lung cancers? Br J Cancer, 90(10), 1905-1911. https://doi.org/10.1038/sj.bjc.6601781

Ruas, J. L., White, J. P., Rao, R. R., Kleiner, S., Brannan, K. T., Harrison, B. C., . . Spiegelman, B. M. (2012). A PGC-1alpha isoform induced by resistance training regulates skeletal muscle hypertrophy. Cell, 151(6), 1319-1331. https://doi.org/10.1016/i.cell.2012.10.050

Safari, R., \& Meuwissen, R. (2015). Practical use of advanced mouse models for lung cancer. Methods Mol Biol, 1267, 93-124. https://doi.org/10.1007/978-1-49392297-0 5

Sakata, I., \& Sakai, T. (2010). Ghrelin cells in the gastrointestinal tract. Int J Pept, 2010. https://doi.org/10.1155/2010/945056

Samuels, S. E., Knowles, A. L., Tilignac, T., Debiton, E., Madelmont, J. C., \& Attaix, D. (2001). Higher skeletal muscle protein synthesis and lower breakdown after 
chemotherapy in cachectic mice. Am J Physiol Regul Integr Comp Physiol, 281(1), R133-139. https://doi.org/10.1152/ajpregu.2001.281.1.R133

Sancak, Y., Bar-Peled, L., Zoncu, R., Markhard, A. L., Nada, S., \& Sabatini, D. M. (2010). Ragulator-Rag complex targets mTORC1 to the lysosomal surface and is necessary for its activation by amino acids. Cell, 141(2), 290-303. https://doi.org/10.1016/i.cell.2010.02.024

Sanchez, A. M., Csibi, A., Raibon, A., Cornille, K., Gay, S., Bernardi, H., \& Candau, R. (2012). AMPK promotes skeletal muscle autophagy through activation of forkhead FoxO3a and interaction with U1k1. J Cell Biochem, 113(2), 695-710. https://doi.org/10.1002/jcb.23399

Sandri, M. (2010). Autophagy in skeletal muscle. FEBS Lett, 584(7), 1411-1416. https://doi.org/10.1016/i.febslet.2010.01.056

Sandri, M., Sandri, C., Gilbert, A., Skurk, C., Calabria, E., Picard, A., . . Goldberg, A. L. (2004). Foxo transcription factors induce the atrophy-related ubiquitin ligase atrogin-1 and cause skeletal muscle atrophy. Cell, 117(3), 399-412. https://doi.org/10.1016/s0092-8674(04)00400-3

Santel, A., Frank, S., Gaume, B., Herrler, M., Youle, R. J., \& Fuller, M. T. (2003). Mitofusin-1 protein is a generally expressed mediator of mitochondrial fusion in mammalian cells. J Cell Sci, 116(Pt 13), 2763-2774. https://doi.org/10.1242/jcs.00479

Sato, S., Gao, S., Puppa, M. J., Kostek, M. C., Wilson, L. B., \& Carson, J. A. (2019). High-Frequency Stimulation on Skeletal Muscle Maintenance in Female Cachectic Mice. Med Sci Sports Exerc, 51(9), 1828-1837. https://doi.org/10.1249/MSS.0000000000001991

Schiaffino, S., \& Reggiani, C. (1994). Myosin isoforms in mammalian skeletal muscle. $J$ Appl Physiol (1985), 77(2), 493-501. https://doi.org/10.1152/jappl.1994.77.2.493

Schiaffino, S., \& Reggiani, C. (2011). Fiber types in mammalian skeletal muscles. Physiol Rev, 91(4), 1447-1531. https://doi.org/10.1152/physrev.00031.2010

Schieke, S. M., Phillips, D., McCoy, J. P., Jr., Aponte, A. M., Shen, R. F., Balaban, R. S., \& Finkel, T. (2006). The mammalian target of rapamycin (mTOR) pathway regulates mitochondrial oxygen consumption and oxidative capacity. J Biol Chem, 281(37), 27643-27652. https://doi.org/10.1074/jbc.M603536200

Scott, J. W., Hawley, S. A., Green, K. A., Anis, M., Stewart, G., Scullion, G. A., . . Hardie, D. G. (2004). CBS domains form energy-sensing modules whose binding of adenosine ligands is disrupted by disease mutations. J Clin Invest, 113(2), 274284. https://doi.org/10.1172/JCI19874

Scott, O. M., Vrbova, G., Hyde, S. A., \& Dubowitz, V. (1985). Effects of chronic low frequency electrical stimulation on normal human tibialis anterior muscle. $J$ Neurol Neurosurg Psychiatry, 48(8), 774-781. https://doi.org/10.1136/jnnp.48.8.774

Sebastian, D., Sorianello, E., Segales, J., Irazoki, A., Ruiz-Bonilla, V., Sala, D., . . . Zorzano, A. (2016). Mfn2 deficiency links age-related sarcopenia and impaired autophagy to activation of an adaptive mitophagy pathway. EMBO J, 35(15), 1677-1693. https://doi.org/10.15252/embj.201593084

Segatto, M., Fittipaldi, R., Pin, F., Sartori, R., Dae Ko, K., Zare, H., . . . Caretti, G. (2017). Epigenetic targeting of bromodomain protein BRD4 counteracts cancer 
cachexia and prolongs survival. Nat Commun, 8(1), 1707.

https://doi.org/10.1038/s41467-017-01645-7

Sengupta, S., Peterson, T. R., \& Sabatini, D. M. (2010). Regulation of the mTOR complex 1 pathway by nutrients, growth factors, and stress. Mol Cell, 4O(2), 310322. https://doi.org/10.1016/j.molcel.2010.09.026

Shimkus, K. L., Jefferson, L. S., Gordon, B. S., \& Kimball, S. R. (2018). Repressors of mTORC1 act to blunt the anabolic response to feeding in the soleus muscle of a cast-immobilized mouse hindlimb. Physiol Rep, 6(20), e13891. https://doi.org/10.14814/phy2.13891

Siegel, I. M. (1989). Update on Duchenne muscular dystrophy. Compr Ther, 15(3), 4552. https://www.ncbi.nlm.nih.gov/pubmed/2650976

Siegel, R. L., Miller, K. D., Fuchs, H. E., \& Jemal, A. (2021). Cancer Statistics, 2021. CA Cancer J Clin, 71(1), 7-33. https://doi.org/10.3322/caac.21654

Siegel, R. L., Miller, K. D., \& Jemal, A. (2020). Cancer statistics, 2020. CA Cancer J Clin, 70(1), 7-30. https://doi.org/10.3322/caac.21590

Silva, K. A., Dong, J., Dong, Y., Dong, Y., Schor, N., Tweardy, D. J., . . Mitch, W. E. (2015). Inhibition of Stat3 activation suppresses caspase-3 and the ubiquitinproteasome system, leading to preservation of muscle mass in cancer cachexia. $J$ Biol Chem, 290(17), 11177-11187. https://doi.org/10.1074/jbc.M115.641514

Soriano, F. X., Liesa, M., Bach, D., Chan, D. C., Palacin, M., \& Zorzano, A. (2006). Evidence for a mitochondrial regulatory pathway defined by peroxisome proliferator-activated receptor-gamma coactivator-1 alpha, estrogen-related receptor-alpha, and mitofusin 2. Diabetes, 55(6), 1783-1791. https://doi.org/10.2337/db05-0509

Squire, J. (2019). Special Issue: The Actin-Myosin Interaction in Muscle: Background and Overview. Int J Mol Sci, 20(22). https://doi.org/10.3390/ijms20225715

Steinberg, G. R., \& Jorgensen, S. B. (2007). The AMP-activated protein kinase: role in regulation of skeletal muscle metabolism and insulin sensitivity. Mini Rev Med Chem, 7(5), 519-526. https://doi.org/10.2174/138955707780619662

Steinberg, G. R., O'Neill, H. M., Dzamko, N. L., Galic, S., Naim, T., Koopman, R., . . . Kemp, B. E. (2010). Whole body deletion of AMP-activated protein kinase $\{$ beta\} 2 reduces muscle AMPK activity and exercise capacity. $J$ Biol Chem, 285(48), 37198-37209. https://doi.org/10.1074/jbc.M110.102434

Stekovic, S., Hofer, S. J., Tripolt, N., Aon, M. A., Royer, P., Pein, L., . . Madeo, F. (2019). Alternate Day Fasting Improves Physiological and Molecular Markers of Aging in Healthy, Non-obese Humans. Cell Metab, 30(3), 462-476 e466. https://doi.org/10.1016/j.cmet.2019.07.016

Stephenson, E. J., Ragauskas, A., Jaligama, S., Redd, J. R., Parvathareddy, J., Peloquin, M. J., . . Bridges, D. (2016). Exposure to environmentally persistent free radicals during gestation lowers energy expenditure and impairs skeletal muscle mitochondrial function in adult mice. Am J Physiol Endocrinol Metab, 310(11), E1003-1015. https://doi.org/10.1152/ajpendo.00521.2015

Streetz, K. L., Tacke, F., Leifeld, L., Wustefeld, T., Graw, A., Klein, C., ... Trautwein, C. (2003). Interleukin 6/gp130-dependent pathways are protective during chronic liver diseases. Hepatology, 38(1), 218-229. https://doi.org/10.1053/jhep.2003.50268 
Sun, R., Zhang, S., Lu, X., Hu, W., Lou, N., Zhao, Y., . . Yang, H. (2016). Comparative molecular analysis of early and late cancer cachexia-induced muscle wasting in mouse models. Oncol Rep, 36(6), 3291-3302. https://doi.org/10.3892/or.2016.5165

Symons, T. B., Sheffield-Moore, M., Mamerow, M. M., Wolfe, R. R., \& Paddon-Jones, D. (2011). The anabolic response to resistance exercise and a protein-rich meal is not diminished by age. $J$ Nutr Health Aging, 15(5), 376-381. https://doi.org/10.1007/s12603-010-0319-z

Szent-Gyorgyi, A. G. (2004). The early history of the biochemistry of muscle contraction. J Gen Physiol, 123(6), 631-641. https://doi.org/10.1085/jgp.200409091

Talbert, E. E., Cuitino, M. C., Ladner, K. J., Rajasekerea, P. V., Siebert, M., Shakya, R., . . Guttridge, D. C. (2019). Modeling Human Cancer-induced Cachexia. Cell Rep, 28(6), 1612-1622 e1614. https://doi.org/10.1016/j.celrep.2019.07.016

Talbot, J., \& Maves, L. (2016). Skeletal muscle fiber type: using insights from muscle developmental biology to dissect targets for susceptibility and resistance to muscle disease. Wiley Interdiscip Rev Dev Biol, 5(4), 518-534. https://doi.org/10.1002/wdev.230

Tardif, N., Klaude, M., Lundell, L., Thorell, A., \& Rooyackers, O. (2013). Autophagiclysosomal pathway is the main proteolytic system modified in the skeletal muscle of esophageal cancer patients. Am J Clin Nutr, 98(6), 1485-1492. https://doi.org/10.3945/ajen.113.063859

Theriault, R., Theriault, G., \& Simoneau, J. A. (1994). Human skeletal muscle adaptation in response to chronic low-frequency electrical stimulation. J Appl Physiol (1985), 77(4), 1885-1889. https://doi.org/10.1152/jappl.1994.77.4.1885

Thomas, M. M., Wang, D. C., D'Souza, D. M., Krause, M. P., Layne, A. S., Criswell, D. S., . . Hawke, T. J. (2014). Muscle-specific AMPK beta1beta2-null mice display a myopathy due to loss of capillary density in nonpostural muscles. FASEB J, 28(5), 2098-2107. https://doi.org/10.1096/fj.13-238972

Tisdale, M. J. (2009). Mechanisms of cancer cachexia. Physiol Rev, 89(2), 381-410. https://doi.org/10.1152/physrev.00016.2008

Tisdale, M. J. (2010). Cancer cachexia. Curr Opin Gastroenterol, 26(2), 146-151. https://doi.org/10.1097/MOG.0b013e3283347e77

Toth, M. J., Callahan, D. M., Miller, M. S., Tourville, T. W., Hackett, S. B., Couch, M. E., \& Dittus, K. (2016). Skeletal muscle fiber size and fiber type distribution in human cancer: Effects of weight loss and relationship to physical function. Clin Nutr, 35(6), 1359-1365. https://doi.org/10.1016/j.clnu.2016.02.016

Toth, M. J., Voigt, T. B., Tourville, T. W., Prior, S. M., Guigni, B. A., Schlosberg, A. V., . . . Dittus, K. (2020). Effect of neuromuscular electrical stimulation on skeletal muscle size and function in patients with breast cancer receiving chemotherapy. $J$ Appl Physiol (1985), 128(6), 1654-1665. https://doi.org/10.1152/japplphysiol.00203.2020

Trommelen, J., Betz, M. W., \& van Loon, L. J. C. (2019). The Muscle Protein Synthetic Response to Meal Ingestion Following Resistance-Type Exercise. Sports Med, 49(2), 185-197. https://doi.org/10.1007/s40279-019-01053-5 
Tsutaki, A., Ogasawara, R., Kobayashi, K., Lee, K., Kouzaki, K., \& Nakazato, K. (2013). Effect of intermittent low-frequency electrical stimulation on the rat gastrocnemius muscle. Biomed Res Int, 2013, 480620. https://doi.org/10.1155/2013/480620

Tzika, A. A., Fontes-Oliveira, C. C., Shestov, A. A., Constantinou, C., Psychogios, N., Righi, V., . . Argiles, J. M. (2013). Skeletal muscle mitochondrial uncoupling in a murine cancer cachexia model. Int J Oncol, 43(3), 886-894. https://doi.org/10.3892/ijo.2013.1998

Tzivion, G., Dobson, M., \& Ramakrishnan, G. (2011). FoxO transcription factors; Regulation by AKT and 14-3-3 proteins. Biochim Biophys Acta, 1813(11), 19381945. https://doi.org/10.1016/j.bbamcr.2011.06.002

Um, J. H., Pendergast, J. S., Springer, D. A., Foretz, M., Viollet, B., Brown, A., . . Chung, J. H. (2011). AMPK regulates circadian rhythms in a tissue- and isoformspecific manner. PLoS One, 6(3), e18450. https://doi.org/10.1371/journal.pone.0018450

Vainshtein, A., \& Hood, D. A. (2015). The regulation of autophagy during exercise in skeletal muscle. J Appl Physiol (1985), jap 0055002015. https://doi.org/10.1152/japplphysiol.00550.2015

van Bokhorst-de van der Schueren, M. A. (2005). Nutritional support strategies for malnourished cancer patients. Eur J Oncol Nurs, 9 Suppl 2, S74-83. https://doi.org/10.1016/j.ejon.2005.09.004

van Dijk, D. P., van de Poll, M. C., Moses, A. G., Preston, T., Olde Damink, S. W., Rensen, S. S., . . . Dejong, C. H. (2015). Effects of oral meal feeding on whole body protein breakdown and protein synthesis in cachectic pancreatic cancer patients. J Cachexia Sarcopenia Muscle, 6(3), 212-221. https://doi.org/10.1002/jcsm.12029

van Dijk, D. P. J., Horstman, A. M. H., Smeets, J. S. J., den Dulk, M., Grabsch, H. I., Dejong, C. H. C., . . . van Loon, L. J. C. (2019). Tumour-specific and organspecific protein synthesis rates in patients with pancreatic cancer. $J$ Cachexia Sarcopenia Muscle, 10(3), 549-556. https://doi.org/10.1002/jcsm.12419

VanderVeen, B. N., Fix, D. K., \& Carson, J. A. (2017). Disrupted Skeletal Muscle Mitochondrial Dynamics, Mitophagy, and Biogenesis during Cancer Cachexia: A Role for Inflammation. Oxid Med Cell Longev, 2017, 3292087. https://doi.org/10.1155/2017/3292087

Vanderveen, B. N., Fix, D. K., Counts, B. R., \& Carson, J. A. (2020). The Effect of Wheel Exercise on Functional Indices of Cachexia in Tumor-bearing Mice. Med Sci Sports Exerc, 52(11), 2320-2330. https://doi.org/10.1249/MSS.0000000000002393

VanderVeen, B. N., Fix, D. K., Montalvo, R. N., Counts, B. R., Smuder, A. J., Murphy, E. A., ... Carson, J. A. (2019). The regulation of skeletal muscle fatigability and mitochondrial function by chronically elevated interleukin-6. Exp Physiol, 104(3), 385-397. https://doi.org/10.1113/EP087429

VanderVeen, B. N., Hardee, J. P., Fix, D. K., \& Carson, J. A. (2018). Skeletal muscle function during the progression of cancer cachexia in the male Apc(Min/+) mouse. J Appl Physiol (1985), 124(3), 684-695. https://doi.org/10.1152/japplphysiol.00897.2017 
Vanhove, K., Graulus, G. J., Mesotten, L., Thomeer, M., Derveaux, E., Noben, J. P., . . . Adriaensens, P. (2019). The Metabolic Landscape of Lung Cancer: New Insights in a Disturbed Glucose Metabolism. Front Oncol, 9, 1215. https://doi.org/10.3389/fonc.2019.01215

Vazeille, C., Jouinot, A., Durand, J. P., Neveux, N., Boudou-Rouquette, P., Huillard, O., . . . Goldwasser, F. (2017). Relation between hypermetabolism, cachexia, and survival in cancer patients: a prospective study in 390 cancer patients before initiation of anticancer therapy. Am J Clin Nutr, 105(5), 1139-1147. https://doi.org/10.3945/ajen.116.140434

Viollet, B., Andreelli, F., Jorgensen, S. B., Perrin, C., Flamez, D., Mu, J., . . V Vaulont, S. (2003). Physiological role of AMP-activated protein kinase (AMPK): insights from knockout mouse models. Biochem Soc Trans, 31(Pt 1), 216-219. https://doi.org/10.1042/bst0310216

Viollet, B., Andreelli, F., Jorgensen, S. B., Perrin, C., Geloen, A., Flamez, D., . . . Vaulont, S. (2003). The AMP-activated protein kinase alpha2 catalytic subunit controls whole-body insulin sensitivity. J Clin Invest, 111(1), 91-98. https://doi.org/10.1172/JCI16567

Viollet, B., Athea, Y., Mounier, R., Guigas, B., Zarrinpashneh, E., Horman, S., . . . Bertrand, L. (2009). AMPK: Lessons from transgenic and knockout animals. Front Biosci (Landmark Ed), 14, 19-44. https://doi.org/10.2741/3229

Viollet, B., Horman, S., Leclerc, J., Lantier, L., Foretz, M., Billaud, M., . . . Andreelli, F. (2010). AMPK inhibition in health and disease. Crit Rev Biochem Mol Biol, 45(4), 276-295. https://doi.org/10.3109/10409238.2010.488215

von Haehling, S., Lainscak, M., Springer, J., \& Anker, S. D. (2009). Cardiac cachexia: a systematic overview. Pharmacol Ther, 121(3), 227-252.

https://doi.org/10.1016/i.pharmthera.2008.09.009

von Walden, F., Liu, C., Aurigemma, N., \& Nader, G. A. (2016). mTOR signaling regulates myotube hypertrophy by modulating protein synthesis, rDNA transcription, and chromatin remodeling. Am J Physiol Cell Physiol, 311(4), C663-C672. https://doi.org/10.1152/ajpcell.00144.2016

Wang, X., Pickrell, A. M., Zimmers, T. A., \& Moraes, C. T. (2012). Increase in muscle mitochondrial biogenesis does not prevent muscle loss but increased tumor size in a mouse model of acute cancer-induced cachexia. PLoS One, 7(3), e33426. https://doi.org/10.1371/journal.pone.0033426

White, J. P., Baltgalvis, K. A., Puppa, M. J., Sato, S., Baynes, J. W., \& Carson, J. A. (2011). Muscle oxidative capacity during IL-6-dependent cancer cachexia. Am J Physiol Regul Integr Comp Physiol, 300(2), R201-211. https://doi.org/10.1152/ajpregu.00300.2010

White, J. P., Baynes, J. W., Welle, S. L., Kostek, M. C., Matesic, L. E., Sato, S., \& Carson, J. A. (2011). The regulation of skeletal muscle protein turnover during the progression of cancer cachexia in the Apc(Min/+) mouse. PLoS One, 6(9), e24650. https://doi.org/10.1371/journal.pone.0024650

White, J. P., Puppa, M. J., Gao, S., Sato, S., Welle, S. L., \& Carson, J. A. (2013). Muscle mTORC1 suppression by IL-6 during cancer cachexia: a role for AMPK. Am J Physiol Endocrinol Metab, 304(10), E1042-1052. https://doi.org/10.1152/ajpendo.00410.2012 
White, J. P., Puppa, M. J., Sato, S., Gao, S., Price, R. L., Baynes, J. W., . . Carson, J. A. (2012). IL-6 regulation on skeletal muscle mitochondrial remodeling during cancer cachexia in the ApcMin/+ mouse. Skelet Muscle, 2, 14. https://doi.org/10.1186/2044-5040-2-14

White, U. A., \& Stephens, J. M. (2011). The gp130 receptor cytokine family: regulators of adipocyte development and function. Curr Pharm Des, 17(4), 340-346. https://doi.org/10.2174/138161211795164202

Williams, A., Sun, X., Fischer, J. E., \& Hasselgren, P. O. (1999). The expression of genes in the ubiquitin-proteasome proteolytic pathway is increased in skeletal muscle from patients with cancer. Surgery, 126(4), 744-749; discussion 749-750. https://www.ncbi.nlm.nih.gov/pubmed/10520924

Williams, J. P., Phillips, B. E., Smith, K., Atherton, P. J., Rankin, D., Selby, A. L., . . Rennie, M. J. (2012). Effect of tumor burden and subsequent surgical resection on skeletal muscle mass and protein turnover in colorectal cancer patients. Am J Clin Nutr, 96(5), 1064-1070. https://doi.org/10.3945/ajcn.112.045708

Witkowski, S., Lovering, R. M., \& Spangenburg, E. E. (2010). High-frequency electrically stimulated skeletal muscle contractions increase p70s6k phosphorylation independent of known IGF-I sensitive signaling pathways. FEBS Lett, 584(13), 2891-2895. https://doi.org/10.1016/j.febslet.2010.05.003

Wolfe, R. R. (2006). The underappreciated role of muscle in health and disease. Am J Clin Nutr, 84(3), 475-482. https://doi.org/10.1093/ajcn/84.3.475

Wu, Z., Puigserver, P., Andersson, U., Zhang, C., Adelmant, G., Mootha, V., . . . Spiegelman, B. M. (1999). Mechanisms controlling mitochondrial biogenesis and respiration through the thermogenic coactivator PGC-1. Cell, 98(1), 115-124. https://doi.org/10.1016/S0092-8674(00)80611-X

Wueest, S., Item, F., Boyle, C. N., Jirkof, P., Cesarovic, N., Ellingsgaard, H., . . Konrad, D. (2014). Interleukin-6 contributes to early fasting-induced free fatty acid mobilization in mice. Am J Physiol Regul Integr Comp Physiol, 306(11), R861867. https://doi.org/10.1152/ajpregu.00533.2013

Xi, Q. L., Zhang, B., Jiang, Y., Zhang, H. S., Meng, Q. Y., Chen, Y., . . Wu, G. H. (2016). Mitofusin-2 prevents skeletal muscle wasting in cancer cachexia. Oncol Lett, 12(5), 4013-4020. https://doi.org/10.3892/ol.2016.5191

Yaffe, M. P. (1999). Dynamic mitochondria. Nat Cell Biol, 1(6), E149-150. https://doi.org/10.1038/14101

Yan, Z., Lira, V. A., \& Greene, N. P. (2012). Exercise training-induced regulation of mitochondrial quality. Exerc Sport Sci Rev, 40(3), 159-164. https://doi.org/10.1097/JES.0b013e3182575599

Yoon, M. S. (2017). mTOR as a Key Regulator in Maintaining Skeletal Muscle Mass. Front Physiol, 8, 788. https://doi.org/10.3389/fphys.2017.00788

Yoshida, K., Taga, T., Saito, M., Suematsu, S., Kumanogoh, A., Tanaka, T., . . . Kishimoto, T. (1996). Targeted disruption of gp130, a common signal transducer for the interleukin 6 family of cytokines, leads to myocardial and hematological disorders. Proc Natl Acad Sci U S A, 93(1), 407-411. https://doi.org/10.1073/pnas.93.1.407

Zhao, L., Hart, S., Cheng, J., Melenhorst, J. J., Bierie, B., Ernst, M., . . . Hennighausen, L. (2004). Mammary gland remodeling depends on gp130 signaling through Stat3 
and MAPK. J Biol Chem, 279(42), 44093-44100.

https://doi.org/10.1074/jbc.M313131200

Zhao, M., Xing, H., Chen, M., Dong, D., \& Wu, B. (2020). Circadian clock-controlled drug metabolism and transport. Xenobiotica, 50(5), 495-505. https://doi.org/10.1080/00498254.2019.1672120

Zhong, X., Pons, M., Poirier, C., Jiang, Y., Liu, J., Sandusky, G. E., . . Z Zimmers, T. A. (2019). The systemic activin response to pancreatic cancer: implications for effective cancer cachexia therapy. J Cachexia Sarcopenia Muscle, 10(5), 10831101. https://doi.org/10.1002/jcsm.12461

Zoncu, R., Efeyan, A., \& Sabatini, D. M. (2011). mTOR: from growth signal integration to cancer, diabetes and ageing. Nat Rev Mol Cell Biol, 12(1), 21-35. https://doi.org/10.1038/nrm3025

Zorzano, A., \& Claret, M. (2015). Implications of mitochondrial dynamics on neurodegeneration and on hypothalamic dysfunction. Front Aging Neurosci, 7 , 101. https://doi.org/10.3389/fnagi.2015.00101

Zurlo, F., Larson, K., Bogardus, C., \& Ravussin, E. (1990). Skeletal muscle metabolism is a major determinant of resting energy expenditure. J Clin Invest, 86(5), 14231427. https://doi.org/10.1172/JCI114857

Zwetsloot, K. A., Westerkamp, L. M., Holmes, B. F., \& Gavin, T. P. (2008). AMPK regulates basal skeletal muscle capillarization and VEGF expression, but is not necessary for the angiogenic response to exercise. J Physiol, 586(24), 6021-6035. https://doi.org/10.1113/jphysiol.2008.159871 
APPENDIX A. ANTIBODY SPECIFICS

Table A-1. Antibody specifics

\begin{tabular}{|c|c|c|c|c|c|c|c|c|c|}
\hline Protein & $\begin{array}{l}\text { Phosphor- } \\
\text { Site }\end{array}$ & Company & Catalogue \# & Protein (ug) & $\begin{array}{c}\text { Blocking } \\
\text { (R.T. 1hr) }\end{array}$ & $1^{\circ}$ Dilution & $1^{\circ}$ Duration & $2^{\circ}$ & $\begin{array}{c}2^{\circ} \text { Dilution } \\
\text { Solution } \\
\text { (R.T. 1hr) }\end{array}$ \\
\hline pAKT & T308 & $\begin{array}{c}\text { Cell } \\
\text { Signaling }\end{array}$ & $13038 \mathrm{~S}$ & 35 & $\begin{array}{c}5 \% \text { TBST- } \\
\text { BSA }\end{array}$ & $\begin{array}{l}1: 500,5 \% \\
\text { TBST-BSA }\end{array}$ & O.N. $4^{\circ}$ & $\begin{array}{c}\text { Anti-rabbit } \\
1: 4000\end{array}$ & $\begin{array}{l}5 \% \text { TBST- } \\
\text { Milk }\end{array}$ \\
\hline AKT & - & $\begin{array}{c}\text { Cell } \\
\text { Signaling }\end{array}$ & $9272 \mathrm{~S}$ & 25 & $\begin{array}{l}5 \% \text { TBST- } \\
\text { Milk }\end{array}$ & $\begin{array}{l}1: 2000,5 \% \\
\text { TBST-Milk }\end{array}$ & R.T. $4 \mathrm{hrs}$ & $\begin{array}{c}\text { Cell } \\
\text { Signaling }\end{array}$ & $\begin{array}{l}5 \% \text { TBST- } \\
\text { Milk }\end{array}$ \\
\hline prpS6 & S240/244 & $\begin{array}{c}\text { Cell } \\
\text { Signaling }\end{array}$ & $5364 \mathrm{~S}$ & 30 & $\begin{array}{l}5 \% \text { TBST- } \\
\text { Milk }\end{array}$ & $\begin{array}{l}\text { 1:1000, } 5 \% \\
\text { TBST-Milk }\end{array}$ & O.N. $4^{\circ}$ & $\begin{array}{c}\text { Anti-rabbit } \\
1: 4000\end{array}$ & $\begin{array}{l}5 \% \text { TBST- } \\
\text { Milk }\end{array}$ \\
\hline rpS6 & - & $\begin{array}{c}\text { Cell } \\
\text { Signaling }\end{array}$ & $2217 \mathrm{~S}$ & 20 & $\begin{array}{l}5 \% \text { TBST- } \\
\text { Milk }\end{array}$ & $\begin{array}{l}\text { 1:2000, } 5 \% \\
\text { TBST-Milk }\end{array}$ & R.T. $4 \mathrm{hrs}$ & $\begin{array}{c}\text { Cell } \\
\text { Signaling }\end{array}$ & $\begin{array}{l}\text { 5\% TBST- } \\
\text { Milk }\end{array}$ \\
\hline pAMPK & $\mathrm{T} 172$ & $\begin{array}{c}\text { Cell } \\
\text { Signaling }\end{array}$ & $2535 \mathrm{~S}$ & 35 & $\begin{array}{l}5 \% \text { TBST- } \\
\text { Milk }\end{array}$ & $\begin{array}{l}\text { 1:1000, } 5 \% \\
\text { TBST-Milk }\end{array}$ & O.N. $4^{\circ}$ & $\begin{array}{c}\text { Anti-rabbit } \\
1: 4000\end{array}$ & $\begin{array}{l}5 \% \text { TBST- } \\
\text { Milk }\end{array}$ \\
\hline AMPK & - & $\begin{array}{c}\text { Cell } \\
\text { Signaling }\end{array}$ & $2603 \mathrm{~S}$ & 30 & $\begin{array}{l}\text { 5\% TBST- } \\
\text { Milk }\end{array}$ & $\begin{array}{l}\text { 1:2000, } 5 \% \\
\text { TBST-Milk }\end{array}$ & R.T. $4 \mathrm{hrs}$ & $\begin{array}{c}\text { Cell } \\
\text { Signaling }\end{array}$ & $\begin{array}{l}\text { 5\% TBST- } \\
\text { Milk }\end{array}$ \\
\hline pFOXO3a & S413 & $\begin{array}{c}\text { Cell } \\
\text { Signaling }\end{array}$ & $8174 \mathrm{~S}$ & 35 & $\begin{array}{c}5 \% \text { TBST- } \\
\text { BSA }\end{array}$ & $\begin{array}{l}1: 500,5 \% \\
\text { TBST-BSA }\end{array}$ & O.N. $4^{\circ}$ & $\begin{array}{c}\text { Anti-rabbit } \\
1: 4000\end{array}$ & $\begin{array}{l}5 \% \text { TBST- } \\
\text { Milk }\end{array}$ \\
\hline FOXO3a & - & $\begin{array}{c}\text { Cell } \\
\text { Signaling }\end{array}$ & $2497 \mathrm{~S}$ & 35 & $\begin{array}{l}5 \% \text { TBST- } \\
\text { Milk }\end{array}$ & $\begin{array}{l}\text { 1:2000, } 5 \% \\
\text { TBST-Milk }\end{array}$ & O.N. $4^{\circ}$ & $\begin{array}{c}\text { Cell } \\
\text { Signaling }\end{array}$ & $\begin{array}{l}\text { 5\% TBST- } \\
\text { Milk }\end{array}$ \\
\hline $\mathrm{pACC}$ & S79 & $\begin{array}{c}\text { Cell } \\
\text { Signaling }\end{array}$ & $3661 \mathrm{~S}$ & 25 & $\begin{array}{l}5 \% \text { TBST- } \\
\text { Milk }\end{array}$ & $\begin{array}{l}\text { 1:1000, 5\% } \\
\text { TBST-Milk }\end{array}$ & R.T. $4 \mathrm{hrs}$ & $\begin{array}{c}\text { Anti-rabbit } \\
1: 4000\end{array}$ & $\begin{array}{l}5 \% \text { TBST- } \\
\text { Milk }\end{array}$ \\
\hline $\mathrm{ACC}$ & - & $\begin{array}{c}\text { Cell } \\
\text { Signaling }\end{array}$ & $3662 \mathrm{~S}$ & 25 & $\begin{array}{l}\text { 5\% TBST- } \\
\text { Milk }\end{array}$ & $\begin{array}{l}\text { 1:2000, } 5 \% \\
\text { TBST-Milk }\end{array}$ & O.N. $4^{\circ}$ & $\begin{array}{c}\text { Cell } \\
\text { Signaling }\end{array}$ & $\begin{array}{l}5 \% \text { TBST- } \\
\text { Milk }\end{array}$ \\
\hline P62 & - & $\begin{array}{c}\text { Cell } \\
\text { Signaling }\end{array}$ & $23214 \mathrm{~S}$ & 30 & $\begin{array}{l}\text { 5\% TBST- } \\
\text { Milk }\end{array}$ & $\begin{array}{l}1: 1000,5 \% \\
\text { TBST-Milk }\end{array}$ & R.T. $4 \mathrm{hrs}$ & $\begin{array}{c}\text { Anti-rabbit } \\
1: 4000\end{array}$ & $\begin{array}{l}5 \% \text { TBST- } \\
\text { Milk }\end{array}$ \\
\hline LC3B & - & $\begin{array}{c}\text { Cell } \\
\text { Signaling }\end{array}$ & $2775 \mathrm{~S}$ & 35 & $\begin{array}{l}5 \% \text { TBST- } \\
\text { Milk }\end{array}$ & $\begin{array}{l}\text { 1:1000, } 5 \% \\
\text { TBST-Milk }\end{array}$ & O.N. $4^{\circ}$ & $\begin{array}{c}\text { Cell } \\
\text { Signaling }\end{array}$ & $\begin{array}{l}\text { 5\% TBST- } \\
\text { Milk }\end{array}$ \\
\hline Beclin-1 & - & $\begin{array}{c}\text { Cell } \\
\text { Signaling }\end{array}$ & $3738 \mathrm{~S}$ & 30 & $\begin{array}{l}5 \% \text { TBST- } \\
\text { Milk }\end{array}$ & $\begin{array}{l}\text { 1:1000, 5\% } \\
\text { TBST-Milk }\end{array}$ & R.T. $4 \mathrm{hrs}$ & $\begin{array}{c}\text { Anti-rabbit } \\
1: 4000\end{array}$ & $\begin{array}{l}5 \% \text { TBST- } \\
\text { Milk }\end{array}$ \\
\hline pSTAT3 & Y705 & $\begin{array}{c}\text { Cell } \\
\text { Signaling }\end{array}$ & $9145 \mathrm{~S}$ & 35 & $\begin{array}{l}5 \% \text { TBST- } \\
\text { Milk }\end{array}$ & $\begin{array}{l}\text { 1:1000, } 5 \% \\
\text { TBST-Milk }\end{array}$ & O.N. $4^{\circ}$ & $\begin{array}{c}\text { Cell } \\
\text { Signaling }\end{array}$ & $\begin{array}{l}5 \% \text { TBST- } \\
\text { Milk }\end{array}$ \\
\hline STAT3 & - & $\begin{array}{c}\text { Cell } \\
\text { Signaling }\end{array}$ & $4904 \mathrm{~S}$ & 30 & $\begin{array}{l}\text { 5\% TBST- } \\
\text { Milk }\end{array}$ & $\begin{array}{l}\text { 1:2000, 5\% } \\
\text { TBST-Milk }\end{array}$ & R.T. $4 \mathrm{hrs}$ & $\begin{array}{c}\text { Anti-rabbit } \\
1: 4000\end{array}$ & $\begin{array}{l}\text { 5\% TBST- } \\
\text { Milk }\end{array}$ \\
\hline pP65 & S536 & $\begin{array}{c}\text { Cell } \\
\text { Signaling }\end{array}$ & & 35 & $\begin{array}{c}5 \% \text { TBST- } \\
\text { BSA }\end{array}$ & $\begin{array}{l}1: 1000,5 \% \\
\text { TBST-BSA }\end{array}$ & O.N. $4^{\circ}$ & $\begin{array}{c}\text { Cell } \\
\text { Signaling }\end{array}$ & $\begin{array}{l}5 \% \text { TBST- } \\
\text { Milk }\end{array}$ \\
\hline P65 & - & $\begin{array}{c}\text { Cell } \\
\text { Signaling }\end{array}$ & $8242 \mathrm{~S}$ & 30 & $\begin{array}{l}5 \% \text { TBST- } \\
\text { Milk }\end{array}$ & $\begin{array}{l}\text { 1:2000, } 5 \% \\
\text { TBST-Milk }\end{array}$ & R.T. $4 \mathrm{hrs}$ & $\begin{array}{c}\text { Anti-rabbit } \\
1: 4000\end{array}$ & $\begin{array}{l}5 \% \text { TBST- } \\
\text { Milk }\end{array}$ \\
\hline pERK & $\mathrm{T} 202 / \mathrm{Y} 204$ & $\begin{array}{c}\text { Cell } \\
\text { Signaling }\end{array}$ & $4370 \mathrm{~S}$ & 30 & $\begin{array}{l}5 \% \text { TBST- } \\
\text { Milk }\end{array}$ & $\begin{array}{l}\text { 1:1000, } 5 \% \\
\text { TBST-Milk }\end{array}$ & R.T. $4 \mathrm{hrs}$ & $\begin{array}{c}\text { Cell } \\
\text { Signaling }\end{array}$ & $\begin{array}{l}5 \% \text { TBST- } \\
\text { Milk }\end{array}$ \\
\hline ERK & - & $\begin{array}{c}\text { Cell } \\
\text { Signaling }\end{array}$ & $9102 \mathrm{~S}$ & 25 & $\begin{array}{l}5 \% \text { TBST- } \\
\text { Milk }\end{array}$ & $\begin{array}{l}\text { 1:2000, } 5 \% \\
\text { TBST-Milk }\end{array}$ & R.T. $4 \mathrm{hrs}$ & $\begin{array}{c}\text { Anti-rabbit } \\
1: 4000\end{array}$ & $\begin{array}{l}5 \% \text { TBST- } \\
\text { Milk }\end{array}$ \\
\hline pP38 & $\mathrm{T} 180 / \mathrm{Y} 182$ & $\begin{array}{c}\text { Cell } \\
\text { Signaling }\end{array}$ & $4511 \mathrm{~S}$ & 30 & $\begin{array}{l}\text { 5\% TBST- } \\
\text { Milk }\end{array}$ & $\begin{array}{l}\text { 1:1000, } 5 \% \\
\text { TBST-Milk }\end{array}$ & R.T. $4 \mathrm{hrs}$ & $\begin{array}{c}\text { Cell } \\
\text { Signaling }\end{array}$ & $\begin{array}{l}5 \% \text { TBST- } \\
\text { Milk }\end{array}$ \\
\hline P38 & - & $\begin{array}{c}\text { Cell } \\
\text { Signaling }\end{array}$ & $9212 \mathrm{~S}$ & 25 & $\begin{array}{l}\text { 5\% TBST- } \\
\text { Milk }\end{array}$ & $\begin{array}{l}1: 2000,5 \% \\
\text { TBST-Milk }\end{array}$ & R.T. $4 \mathrm{hrs}$ & $\begin{array}{c}\text { Anti-rabbit } \\
1: 4000\end{array}$ & $\begin{array}{l}5 \% \text { TBST- } \\
\text { Milk }\end{array}$ \\
\hline gp130 & - & $\begin{array}{c}\text { Cell } \\
\text { Signaling }\end{array}$ & $3732 S$ & 35 & $\begin{array}{l}5 \% \text { TBST- } \\
\text { Milk }\end{array}$ & $\begin{array}{l}\text { 1:1000, } 5 \% \\
\text { TBST-Milk }\end{array}$ & O.N. $4^{\circ}$ & $\begin{array}{c}\text { Cell } \\
\text { Signaling }\end{array}$ & $\begin{array}{l}5 \% \text { TBST- } \\
\text { Milk }\end{array}$ \\
\hline pULK1 & S555 & $\begin{array}{c}\text { Cell } \\
\text { Signaling }\end{array}$ & $5869 \mathrm{~S}$ & 35 & $\begin{array}{c}5 \% \text { TBST- } \\
\text { BSA }\end{array}$ & $\begin{array}{l}1: 500,5 \% \\
\text { TBST-BSA }\end{array}$ & O.N. $4^{\circ}$ & $\begin{array}{c}\text { Anti-rabbit } \\
1: 4000\end{array}$ & $\begin{array}{l}5 \% \text { TBST- } \\
\text { Milk }\end{array}$ \\
\hline Atrogin-1 & - & $\begin{array}{c}\text { ECM } \\
\text { Biosciences }\end{array}$ & AP2041 & 30 & $\begin{array}{l}5 \% \text { TBST- } \\
\text { Milk }\end{array}$ & $\begin{array}{l}\text { 1:1000, } 5 \% \\
\text { TBST-Milk }\end{array}$ & O.N. $4^{\circ}$ & $\begin{array}{c}\text { Cell } \\
\text { Signaling }\end{array}$ & $\begin{array}{l}5 \% \text { TBST- } \\
\text { Milk }\end{array}$ \\
\hline MuRF-1 & - & $\begin{array}{c}\text { ECM } \\
\text { Biosciences }\end{array}$ & MP3401 & 30 & $\begin{array}{l}5 \% \text { TBST- } \\
\text { Milk }\end{array}$ & $\begin{array}{l}\text { 1:1000, } 5 \% \\
\text { TBST-Milk }\end{array}$ & O.N. $4^{\circ}$ & $\begin{array}{c}\text { Anti-rabbit } \\
1: 4000\end{array}$ & $\begin{array}{l}5 \% \text { TBST- } \\
\text { Milk }\end{array}$ \\
\hline PGC1alpha & - & Abcam & ab106814 & 30 & $\begin{array}{l}5 \% \text { TBST- } \\
\text { Milk }\end{array}$ & $\begin{array}{l}\text { 1:1000, } 5 \% \\
\text { TBST-Milk }\end{array}$ & O.N. $4^{\circ}$ & $\begin{array}{c}\text { Cell } \\
\text { Signaling }\end{array}$ & $\begin{array}{l}5 \% \text { TBST- } \\
\text { Milk }\end{array}$ \\
\hline pDRP1 & S616 & $\begin{array}{c}\text { Cell } \\
\text { Signaling }\end{array}$ & $3455 \mathrm{~S}$ & 35 & $\begin{array}{l}5 \% \text { TBST- } \\
\text { Milk }\end{array}$ & $\begin{array}{l}\text { 1:1000, } 5 \% \\
\text { TBST-Milk }\end{array}$ & O.N. $4^{\circ}$ & $\begin{array}{c}\text { Anti-rabbit } \\
1: 4000\end{array}$ & $\begin{array}{l}5 \% \text { TBST- } \\
\text { Milk }\end{array}$ \\
\hline DRP1 & - & $\begin{array}{c}\text { Cell } \\
\text { Signaling }\end{array}$ & $5391 \mathrm{~S}$ & 30 & $\begin{array}{l}5 \% \text { TBST- } \\
\text { Milk }\end{array}$ & $\begin{array}{l}\text { 1:2000, } 5 \% \\
\text { TBST-Milk }\end{array}$ & R.T. 4hrs & $\begin{array}{c}\text { Cell } \\
\text { Signaling }\end{array}$ & $\begin{array}{l}5 \% \text { TBST- } \\
\text { Milk }\end{array}$ \\
\hline FIS1 & - & Sigma & HPA017430 & 35 & $\begin{array}{l}5 \% \text { TBST- } \\
\text { Milk }\end{array}$ & $\begin{array}{l}\text { 1:1000, } 5 \% \\
\text { TBST-Milk }\end{array}$ & R.T. $4 \mathrm{hrs}$ & $\begin{array}{c}\text { Anti-rabbit } \\
1: 4000\end{array}$ & $\begin{array}{l}5 \% \text { TBST- } \\
\text { Milk }\end{array}$ \\
\hline OPA1 & - & $\begin{array}{c}\text { Cell } \\
\text { Signaling }\end{array}$ & $80471 \mathrm{~S}$ & 35 & $\begin{array}{l}\text { 5\% TBST- } \\
\text { Milk }\end{array}$ & $\begin{array}{l}1: 1000,5 \% \\
\text { TBST-Milk }\end{array}$ & R.T. $4 \mathrm{hrs}$ & $\begin{array}{c}\text { Cell } \\
\text { Signaling }\end{array}$ & $\begin{array}{l}5 \% \text { TBST- } \\
\text { Milk }\end{array}$ \\
\hline OXPHOS & - & Abcam & ab1 10413 & 25 & $\begin{array}{l}5 \% \text { TBST- } \\
\text { Milk }\end{array}$ & $\begin{array}{l}1: 4000,5 \% \\
\text { TBST-Milk }\end{array}$ & O.N. $4^{\circ}$ & $\begin{array}{c}\text { Anti-rabbit } \\
1: 4000\end{array}$ & $\begin{array}{l}5 \% \text { TBST- } \\
\text { Milk }\end{array}$ \\
\hline $\begin{array}{l}\text { Puromycin } \\
\text { Incorporation }\end{array}$ & - & Millipore & MABE343 & 30 & $\begin{array}{l}5 \% \text { TBST- } \\
\text { Milk }\end{array}$ & $\begin{array}{l}\text { 1:4000, } 5 \% \\
\text { TBST-Milk }\end{array}$ & O.N. $4^{\circ}$ & $\begin{array}{c}\text { Cell } \\
\text { Signaling }\end{array}$ & $\begin{array}{l}5 \% \text { TBST- } \\
\text { Milk }\end{array}$ \\
\hline OXPHOS & - & Abcam & ab110413 & 25 & $\begin{array}{l}5 \% \text { TBST- } \\
\text { Milk }\end{array}$ & $\begin{array}{l}\text { 1:4000, } 5 \% \\
\text { TBST-Milk }\end{array}$ & O.N. $4^{\circ}$ & $\begin{array}{c}\text { Anti-rabbit } \\
1: 4000 \\
\text { Anti-Mouse }\end{array}$ & $\begin{array}{l}5 \% \text { TBST- } \\
\text { Milk }\end{array}$ \\
\hline $\begin{array}{l}\text { Puromycin } \\
\text { Incorporation }\end{array}$ & - & Millipore & MABE343 & 30 & $\begin{array}{l}5 \% \text { TBST- } \\
\text { Milk }\end{array}$ & $\begin{array}{l}\text { 1:4000, } 5 \% \\
\text { TBST-Milk }\end{array}$ & R.T. $4 \mathrm{hrs}$ & $\begin{array}{c}1: 4000 \\
\text { Cell } \\
\text { Signaling }\end{array}$ & $\begin{array}{l}5 \% \text { TBST- } \\
\text { Milk }\end{array}$ \\
\hline OXPHOS & - & Abcam & ab110413 & 25 & $\begin{array}{l}5 \% \text { TBST- } \\
\text { Milk }\end{array}$ & $\begin{array}{l}1: 4000,5 \% \\
\text { TBST-Milk }\end{array}$ & O.N. $4^{\circ}$ & $\begin{array}{c}\text { Anti-rabbit } \\
1: 4000\end{array}$ & \\
\hline
\end{tabular}




\section{APPENDIX B. FEEDING AND FASTING SUPPLEMENTAL DATA}

A.

$$
\begin{aligned}
& \text { PBS } \\
& \text { LLC }
\end{aligned}
$$

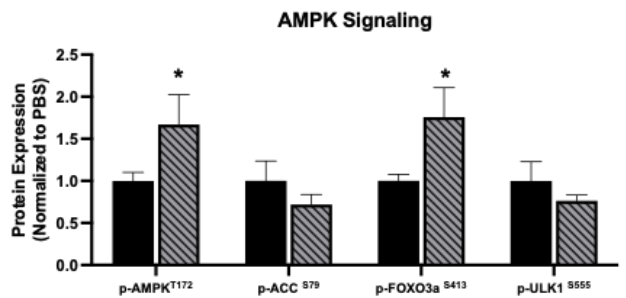

$$
\begin{aligned}
& \text { C. } \\
& \text { 四 LLC }
\end{aligned}
$$

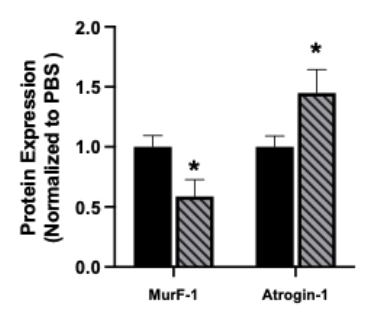

E.

$$
\text { U LLC }
$$

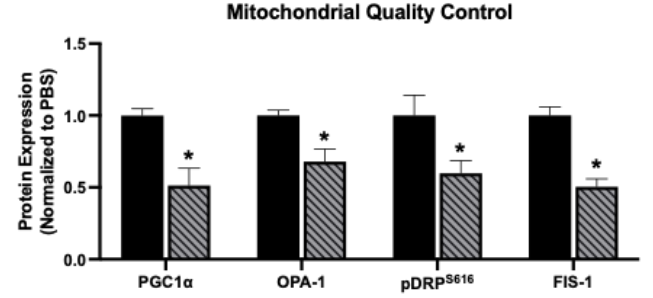

B.

$$
\text { PBS }
$$

LLC

Autophagy Associated Siganling

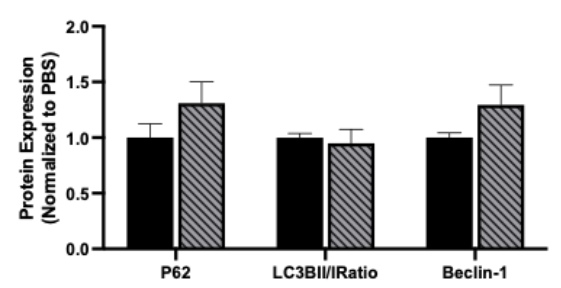

D.

$$
\text { 四 LLC }
$$

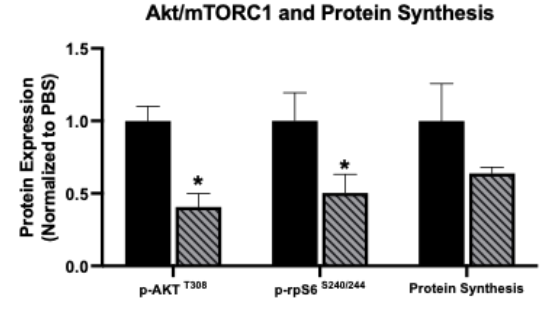

Figure B-1. The effect of LLC on skeletal muscle signaling in male mice following an overnight $12 \mathrm{hr}$ fast

Notes: Data are presented as mean \pm SEM. A) AMPK associated signaling B) Autophagy associated signaling. C) E3 Ligase protein expression. D) Akt/mTORC1 signaling and protein synthesis protein expression E) Mitochondrial quality control protein expression Phosphorylation proteins were normalized to total protein expression. Protein expression was normalized to PBS mice. Protein expression was analyzed in white gastrocnemius muscle. $\mathrm{N}=7-9$ per group. Unpaired T-test were used to compare PBS and LLC male mice. * Different from PBS. 
A.

A. ${ }^{\text {Fast }}$ Fed

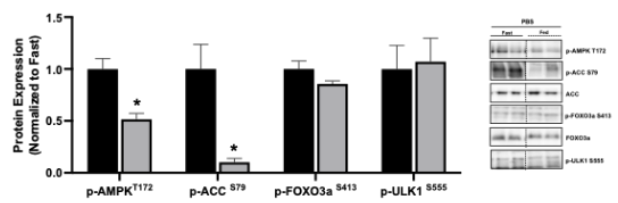

B.
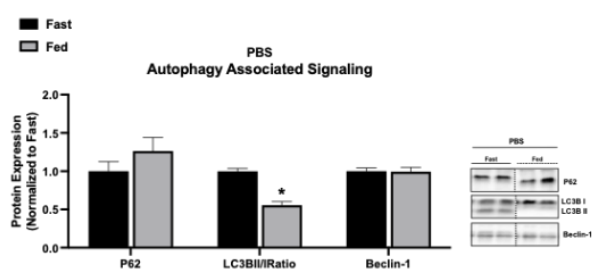

C.

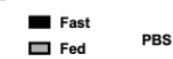

D.
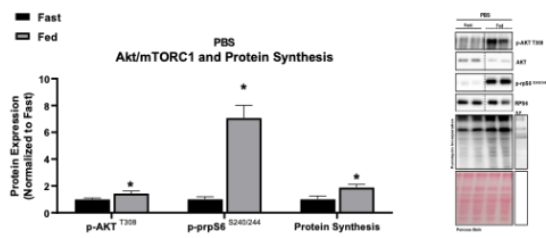

E.

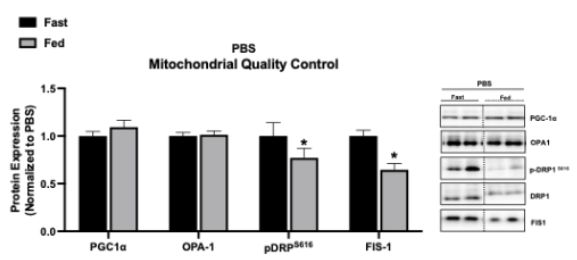

Figure B-2. The effect of feeding in PBS male mice following an overnight $12 \mathrm{hr}$ fast

Notes: Data are presented as mean \pm SEM. A) AMPK associated signaling B) Autophagy associated signaling C) E3 ligase protein expression D) Akt/mTORC1 and protein synthesis protein expression. E) Mitochondrial quality control protein expression. Phosphorylation proteins were normalized to total protein expression. Protein expression was normalized to Fast mice. Protein expression was analyzed in white gastrocnemius muscle. $\mathrm{N}=7-8$ per group. Unpaired T-test were used to compare AMPK WT Fast and Fed mice 
A.

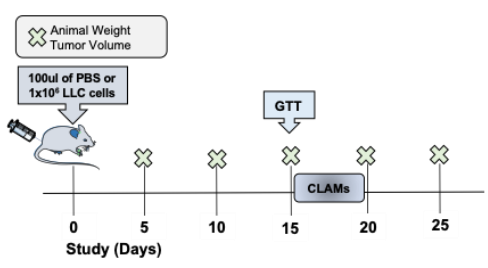

B.

C.
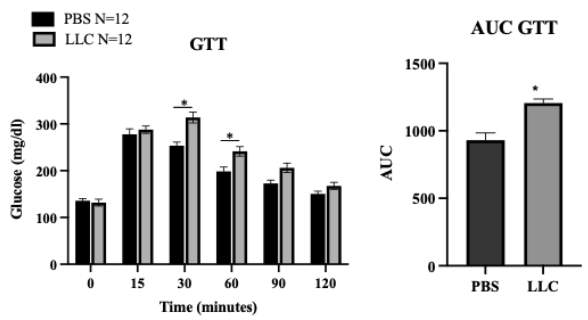

D.

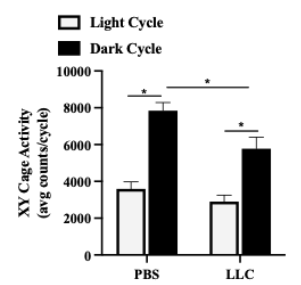

E.
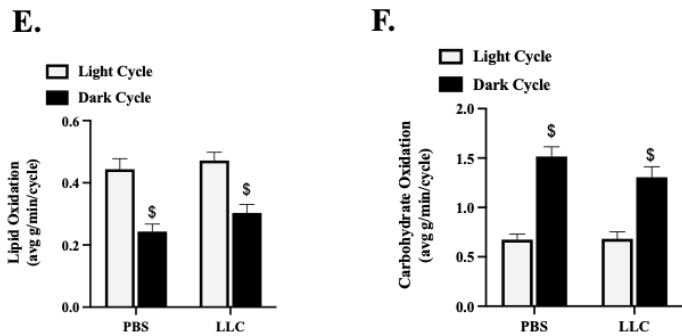

Figure B-3. Early onset whole body metabolism and physical activity in a subset of LLC tumor baring mice

Notes: Data are presented as mean \pm SEM. A) Study Design B) Glucose overtime during glucose tolerance test. C) Area under the curve from glucose tolerance test. D) Average cage activity during the light and dark in PBS and LLC male mice. E) Average lipid oxidation during the light and dark in PBS and LLC male mice. F) Average carbohydrate oxidation during the light and dark in PBS and LLC male mice. $\mathrm{N}=5-7$ per group. Twoway repeated measures ANOVA were used. Unpaired t-test was used to compared GTT AUC in PBS and LLC mice. * Groups are different. \$ Main effect of Cycle. 
Table B-1. Associations of early onset systemic metabolism and physical activity to indices ot cachexia and tumor development

\begin{tabular}{|c|c|c|c|}
\hline \multirow[b]{2}{*}{ Factor 1 (early-onset) } & \multirow[b]{2}{*}{ Factor 2 (late stage) } & \multicolumn{2}{|c|}{ LLC } \\
\hline & & $\mathbf{R}^{2}$ & $\mathbf{p}$ \\
\hline \multirow[t]{7}{*}{ GTT Glucose AUC $(\mathrm{N}=12)$} & $\mathrm{BW} \boldsymbol{\Delta} \mathrm{d} 10$ & -0.518 & 0.084 \\
\hline & BW $\Delta \mathrm{d} 0$ & -0.423 & 0.171 \\
\hline & Gastrocnemius Mass & 0.175 & 0.587 \\
\hline & eWAT & -0.579 & $0.048 *$ \\
\hline & Tumor Mass & 0.261 & 0.413 \\
\hline & Tumor Growth Rate & -0.036 & 0.911 \\
\hline & Plasma IL-6 & 0.093 & 0.773 \\
\hline \multirow[t]{7}{*}{ Lipid Oxidation ( $\mathrm{N}=14)$} & $\mathrm{BW} \Delta \mathrm{d} 10$ & -0.131 & 0.654 \\
\hline & $\mathrm{BW} \Delta \mathrm{d} 0$ & -0.064 & 0.827 \\
\hline & Gastrocnemius Mass & -0.027 & 0.928 \\
\hline & eWAT & -0.443 & 0.113 \\
\hline & Tumor Mass & 0.346 & 0.225 \\
\hline & Tumor Growth Rate & 0.155 & 0.600 \\
\hline & Plasma IL-6 & 0.153 & 0.601 \\
\hline \multirow[t]{7}{*}{ Carbohydrate Oxidation $(\mathrm{N}=14)$} & $\mathrm{BW} \Delta \mathrm{d} 10$ & -0.342 & 0.231 \\
\hline & BW $\Delta \mathrm{d} 0$ & -0.269 & 0.353 \\
\hline & Gastrocnemius Mass & -0.369 & 0.195 \\
\hline & eWAT & -0.117 & 0.689 \\
\hline & Tumor Mass & 0.330 & 0.249 \\
\hline & Tumor Growth Rate & -0.106 & 0.718 \\
\hline & Plasma IL-6 & 0.437 & 0.118 \\
\hline \multirow[t]{7}{*}{ Physical Activity $(\mathrm{N}=14)$} & $\mathrm{BW} \boldsymbol{\Delta} \mathrm{d} 10$ & 0.029 & 0.922 \\
\hline & $\mathrm{BW} \Delta \mathrm{d} 0$ & 0.103 & 0.726 \\
\hline & Gastrocnemius Mass & 0.784 & $<0.001 *$ \\
\hline & eWAT & 0.402 & $<0.001 *$ \\
\hline & Tumor Mass & -0.487 & 0.078 \\
\hline & Tumor Growth Rate & 0.187 & 0.522 \\
\hline & Plasma IL-6 & -0.656 & $0.011 *$ \\
\hline
\end{tabular}

Notes: Associations of early-onset systemic metabolism and physical activity to indices of cachexia and tumor development. Data are presented as mean \pm SEM. $N=5-7$ per group. Pearson spearman correlation were used to association of systemic metabolism and physical activity to indices of cachexia and tumor growth rate. Abbreviations: LLC: Lewis lung carcinoma. * Statistically significant $\mathrm{p}<0.05$. 
Table B-2. Associations of early onset systemic metabolism and physical activity to AMPK association signaling in fast and fed LLC mice

\begin{tabular}{|c|c|c|c|c|c|}
\hline \multirow[b]{2}{*}{ Factor 1} & \multirow[b]{2}{*}{ Factor 2} & \multicolumn{2}{|c|}{ Fast } & \multicolumn{2}{|c|}{ Fed } \\
\hline & & $\mathbf{R}^{2}$ & p & $\mathbf{R}^{2}$ & $\mathbf{p}$ \\
\hline \multirow[t]{4}{*}{ pAMPK $^{\text {T172 }}$} & GTT Glucose AUC & -0.164 & 0.792 & -0.672 & 0.328 \\
\hline & Lipid Oxidation & 0.823 & 0.087 & 0.032 & 0.946 \\
\hline & Carbohydrate Oxidation & 0.141 & 0.821 & 0.214 & 0.644 \\
\hline & Physical Activity & -0.056 & 0.928 & 0.186 & 0.690 \\
\hline \multirow[t]{4}{*}{ pULK $^{\mathrm{S} 555}$} & GTT Glucose AUC & -0.173 & 0.826 & -0.760 & 0.241 \\
\hline & Lipid Oxidation & -0.980 & $0.021 *$ & -0.179 & 0.701 \\
\hline & Carbohydrate Oxidation & -0.288 & 0.712 & -0.623 & 0.135 \\
\hline & Physical Activity & 0.339 & 0.661 & 0.805 & $0.029 *$ \\
\hline \multirow[t]{4}{*}{ 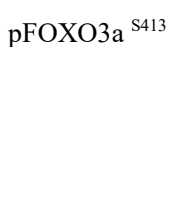 } & GTT Glucose AUC & 0.453 & 0.444 & -0.613 & 0.271 \\
\hline & Lipid Oxidation & -0.256 & 0.677 & -0.178 & 0.703 \\
\hline & Carbohydrate Oxidation & -0.897 & $0.039 *$ & -0.521 & 0.231 \\
\hline & Physical Activity & 0.538 & 0.349 & 0.355 & 0.435 \\
\hline
\end{tabular}

Notes: Data are presented as mean \pm SEM. Pearson spearman correlation were used to association of systemic metabolism and physical activity to indices of cachexia and tumor growth rate. Abbreviations: LLC: Lewis lung carcinoma. * Statistically significant $\mathrm{p}<0.05$. 
A.

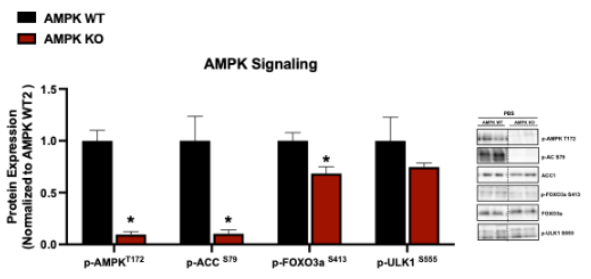

B.

C.

Dampkr
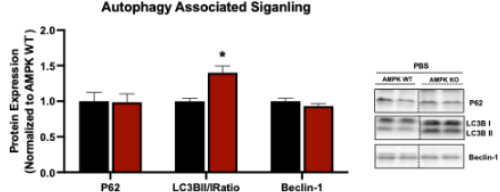

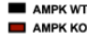

D.

- миркит

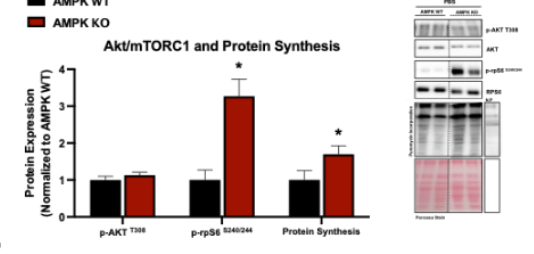

E.

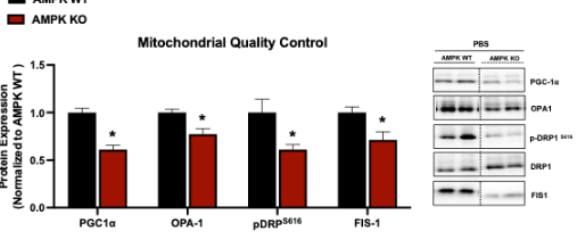

Figure B-4. The effect of muscle-specific AMPK loss in PBS male mice following an overnight $12 \mathrm{hr}$ fast

Notes: Data are presented as mean \pm SEM. A) AMPK associated signaling B) Autophagy associated signaling C) E3 ligase protein expression D) Akt/mTORC1 and protein synthesis protein expression. E) Mitochondrial quality control protein expression. Phosphorylation proteins were normalized to total protein expression. Protein expression was normalized to AMPK WT mice. $\mathrm{N}=7-10$ per group. Unpaired T-test were used to compare AMPK WT and AMPK KO mice. 

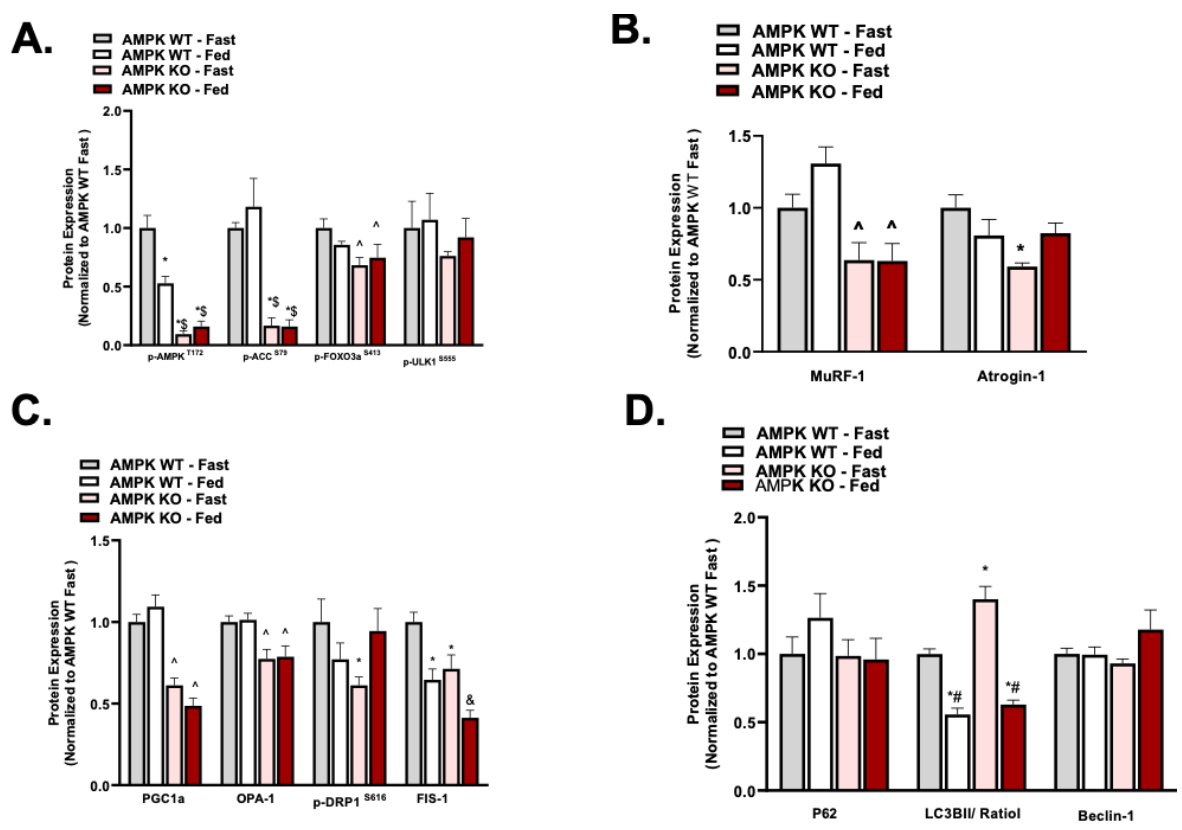

Figure B-5. The effect of muscle-specific AMPK loss on the feeding regulation of muscle protein turnover in PBS male mice

Notes: Data are presented as mean \pm SEM. A) AMPK associated signaling B) E3 Ligase protein expression. C) Mitochondrial quality control protein expression. D) Autophagy associated signaling. Phosphorylation proteins were normalized to total protein expression. Protein expression was normalized to Fast mice. Protein expression was analyzed in white gastrocnemius muscle. N=7-10 per group. Two-way ANOVA was used to compared AMPK WT and AMPK KO mice in the fast and fed condition. * Different from AMPK WT -Fast, \$ Different from AMPK WT -Fed, \&Different from all groups, \#Different from AMPK KO Fast, ${ }^{\wedge}$ Main Effect of AMPK KO. 
A.

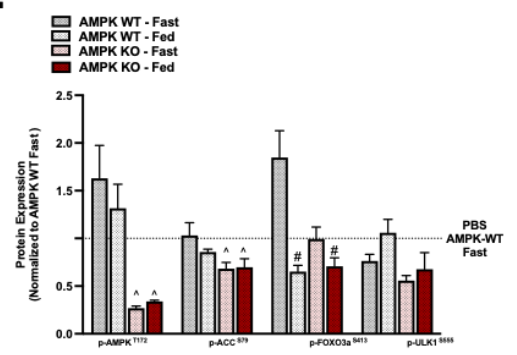

C.

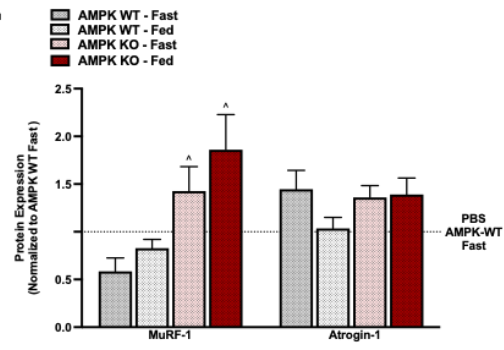

B.

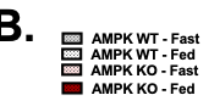

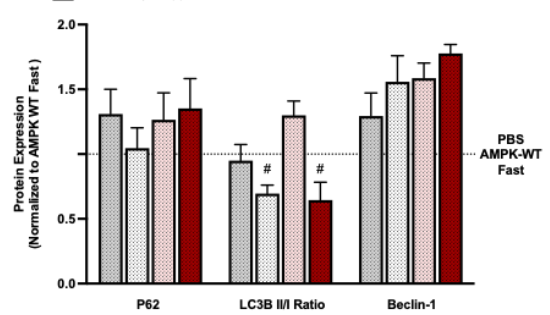

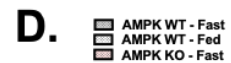

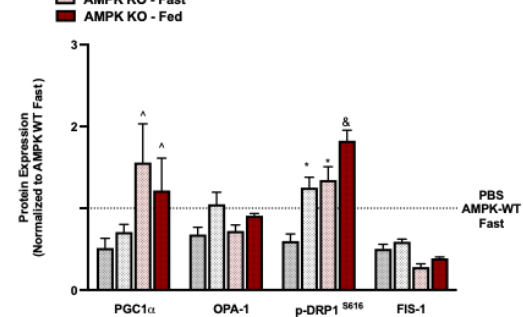

Figure B-6. The effect of muscle-specific AMPK loss on the feeding regulation of muscle protein turnover in LLC male mice

Notes: Data are presented as mean \pm SEM. A) AMPK associated signaling B) Autophagy associated signaling. C) E3 Ligase protein expression. D) Mitochondrial quality control protein expression. Phosphorylation proteins were normalized to total protein expression. Protein expression was normalized to PBS mice. Protein expression was analyzed in white gastrocnemius muscle. $\mathrm{N}=8-11$ per group. Two-way ANOVAs were used to compare AMPK WT and AMPK KO LLC mice in the fast and fed condition. Main effect of KO, \#Main Effect of Feeding, *Different from AMPK WT Fast, \&Different from all groups. 
A.

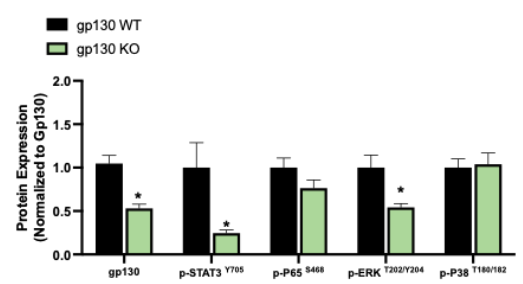

C.

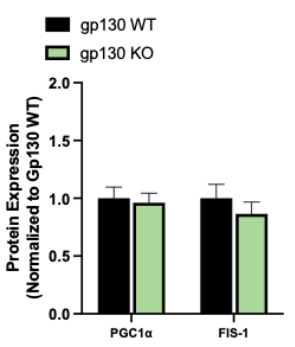

B.

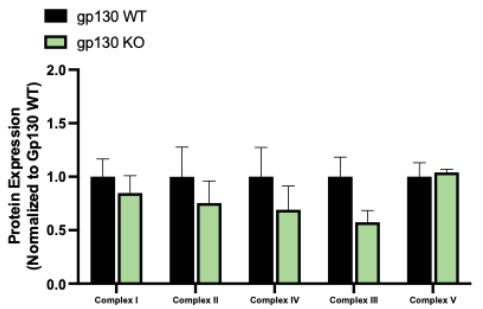

D.

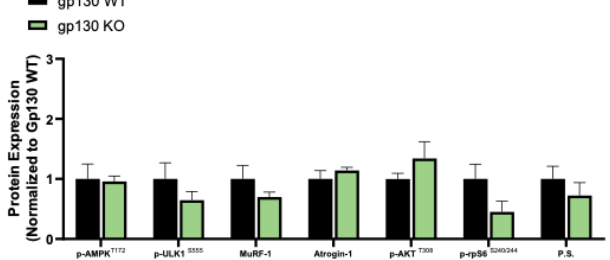

Figure B-7. The effect of muscle-specific gp130 loss on skeletal muscle protein turnover in PBS male mice following an overnight $12 \mathrm{hr}$ fast

Notes: Data are presented as mean \pm SEM. A) gp130 associated signaling B) Mitochondrial complex protein expression C) PGC1 $\alpha$ and FIS1 protein expression D) Marker of skeletal muscle protein turnover. Protein expression was analyzed in white gastrocnemius muscle. Phosphorylation proteins were normalized to total protein expression. Protein expression was normalized to gp130 WT mice. N=7 per group. Unpaired T-test were used to compare gp130 WT and gp130 KO mice. 


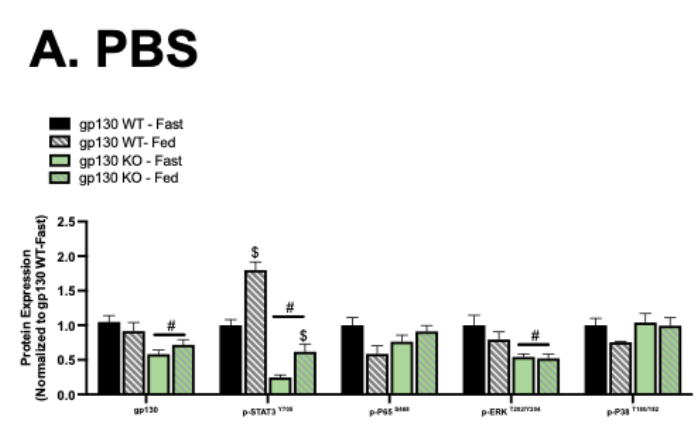

C. LLC Mice

$$
\begin{aligned}
& \text { gp130 WT - Fast } \\
& \text { gp130 WT- Fed } \\
& \text { gp130 KO - Fast } \\
& \text { gp130 KO - Fed }
\end{aligned}
$$

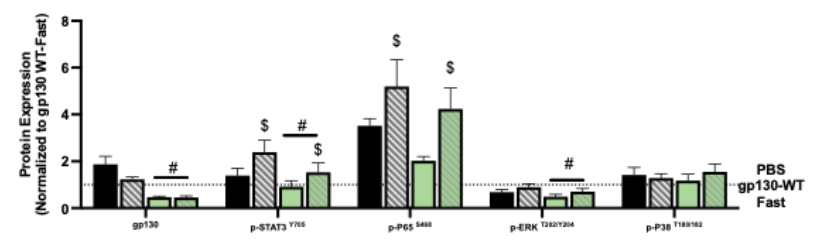

B. PBS

gp130 WT - Fast

gp130 KO - Fast

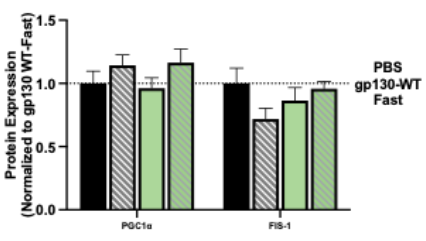

D. LLC Mice

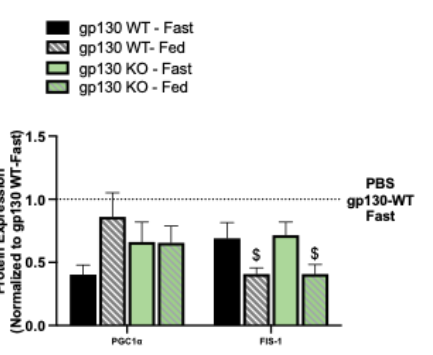

Figure B-8. The effect of muscle-specific gp130 loss on skeletal muscle inflammatory signaling and mitochondrial quality control in PBS and LLC male mice following $1 \mathrm{hr}$ ad libitum feeding

Notes: Data are presented as mean \pm SEM. A) gp130 associated signaling B) Mitochondrial complex protein expression C) PGC1 $\alpha$ and FIS1 protein expression D) Marker of skeletal muscle protein turnover. Protein expression was analyzed in white gastrocnemius muscle. Phosphorylation proteins were normalized to total protein expression. Protein expression was normalized to PBS gp130 WT- Fast mice. N=7-11 per group. Two-Way ANOVA was used to compare within each treatment group (PBS or LLC) gp130 loss in the fast and fed condition. \# Main effect of gp130, \$ Main effect of feeding. 


\section{APPENDIX C. EXTENDED METHODS}

\section{Tamoxifen Preparation and Administration}

1. Dissolve $85 \mathrm{mg}$ of tamoxifen (Sigma T5648) in $1 \mathrm{ml}$ ethanol (200 proof). Note: The OpenWetWare site (http://openwetware.org/wiki/Tamoxifen administration to mice) indicates tamoxifen is soluble at $100 \mathrm{mg} / \mathrm{ml}$ but I have never been able to get more than 85 $\mathrm{mg} / \mathrm{ml}$ consistently into solution.

2. Add 150 ul tamoxifen suspension to 850 ul sunflower seed oil (Sigma S5007) and vortex (highest setting) until mixture becomes clear. The tamoxifen is now at $12.75 \mathrm{mg} / \mathrm{ml}$.

Note: A 15:85 ethanol: sunflower mixture was the best I could achieve before to two phases would spontaneously separate after vortexing.

3. This mixture is stored for up to 1 week at $4^{\circ} \mathrm{C}$.

4. Prior to use, each aliquot (I typically make $1 \mathrm{ml}$ aliquots) is sonicated for 20 minutes using a Branson Ultra-sonicator.

Note: My understanding is that this step helps to emulsify the solution, possibility improving tamoxifen "bioavailability", but I have not read anything to directly support this idea.

5. Following sonication, load each aliquot into a $1 \mathrm{ml}$ tuberculin syringe (BD ref.\# 309602) with a $27 \mathrm{~g} \mathrm{1/2} \mathrm{inch} \mathrm{needle} \mathrm{(BD} \mathrm{305109).}$

6. Administer $2 \mathrm{mg}$ of tamoxifen by IP injection (approximately $160 \mathrm{ul}$ ) or equivalent volume of vehicle (15\%ethanol in sunflower seed oil).

7. We do 5 consecutive days of injections followed by a two-week washout period.

\section{Feeding Stimulus}

When mice achieve criteria of study endpoint, mice were fasted during the dark cycle for $12 \mathrm{hrs}$. At the end of the dark cycle, mice were given access to a standard rodent chow food pellet for $1 \mathrm{hr}$. Food pellet weight, plasma insulin, and blood glucose were measured pre and post feeding stimulus for feeding validation. Ad libitum access to food pellet for $1 \mathrm{hr}$ has been consistently shown to induce mTORC1 and protein synthesis (Gordon et al., 2015). In aim 1 and aim 2, mice were sacrificed immediately after the $1 \mathrm{hr}$ ad libitum access to a food pellet. In aim 3, mice were given access for a food pellet for $1 \mathrm{hr}$ ad libitum after the overnight $12 \mathrm{hr}$ fast. Mice then underwent 30 minutes of low frequency electrical stimulation and sacrificed $3 \mathrm{hrs}$ post stimulation. Therefore, aim 3 mice were sacrificed $3 \mathrm{hrs}$ and 30 minutes post feeding.

1. Place mouse in fresh cage single housed at the start of fasting.

2. Bring mouse to $\mathrm{Rm}$. 336 and let acclimate for 30 minutes. 
3. Make sure the cage has a water bottle (preferred to be placed in the night before during the fast).

4. Complete pre pellet weight, blood glucose, and plasma insulin measurements.

5. Place the food pellet at the bottom of the cage and confirm that the mouse initiates eating the pellet.

6. Complete post pellet weight, blood glucose, and plasma insulin measurements.

\section{CLAMs Cages}

\section{Placing Mice in CLAMs Unit:}

i. MRI Protocol:

1. Each mouse will be weighed prior to analysis by Echo MRI.

2. The Echo MRI machine will be calibrated with corn oil in the calibration tube.

3. Each mouse will be processed through the Echo MRI individually by insertion into a $48 \mathrm{~cm} \mathrm{x} 5.4 \mathrm{~cm}$ cylindrical MRI tube.

4. Once in the tube, the MRI process inside the machine lasts up to 3.2 minutes.

5. Once the MRI is completed, each mouse will be returned to its home cage.

b. General Protocol:

i. Animals will be housed individually for up to one week, in sight of other animals.

ii. Animals will always have access to water and will not be fasted longer than 12 consecutive hours.

iii. Each cage will be labeled with the appropriate mouse number a second hard copy recorded will be kept, and a third recorded will be noted on the CLAMs computer program.

iv. Four 5-ounce scoops of Harlan Teklad 7087C soft cob bedding will be added to each cage. An additional 5-ounce scoop of dirty bedding from the mouse's home cage will be added to the clean cage $(26.6 \mathrm{~cm} \times 20.7 \mathrm{~cm} .14 .3 \mathrm{~cm})$ in efforts to comfort the mouse in its new environment.

v. Each cage part will be placed beside each other.

vi. A stationary metal cylinder will be attached to the plastic connector on the lid.

vii. Feeding containers will be filled with the appropriate diet. When full, each container holds approximately $80 \mathrm{~g}$ of food. A full container will last a mouse 10-14 days.

viii. Feeding containers will be hooked into the motorized feeding monitor facing forward. The motorized feeding monitor/feeding 
container combination will be attached to the cage lid-again will care taken to ensure the feeder is facing forward.

ix. A water bottle containing approximately $125 \mathrm{~mL}$ of water will be screwed into the appropriate place on the lid.

x. Mice will be removed from their LACU cages and caged singularly in a metabolic cage with new and old bedding as outlined above (Step 3).

xi. A cage brace will be added to the center of the cage and clasped into place. After a change of gloves, the cage will be placed into the CLAMs incubator and all wires and tubes will be connected. To prevent any direct contact between the contents of the cage and the inside of the incubator, no cages will be opened prior to removal from the incubator.

xii. The CLAMs will be calibrated for $\mathrm{CO} 2$ and $\mathrm{O} 2$ (per computer program protocol).

c. Before run checklist.

i. Each cage has bedding.

ii. Each cage has a water bottle with liquid attached and screwed in tightly. Tap the bottles to ensure that there are no clogs and water is free flowing.

iii. Each cage has a cage brace, Wheels are set to free or locked. Check all wires and cords: Air connection, Fan plug, Wheel plug, Feeding monitor ethernet cord.

iv. All feeding containers are facing forward (are accessible to the mice).

v. All feeding containers are facing forward (are accessible to the mice). Check after first cycle (up to approximately 32 minutes).

vi. All airflow is within the acceptable range (approximately 0.50). Check after the first cycle (up to approximately 32 minutes). If the airflow tubing is properly connected. If the airflow is high (highlighted red and above 0.85 ) make sure there are no blocks in the airflow tubing.

d. Daily Checklist.

i. Check water bottles have liquid in them. If water bottles have leaked, ref-fill with deionized water. If bedding is wet, change bedding.

ii. Check that food containers are turned forward (accessible to mice) and have sufficient food. Re-fill empty containers and turn feeders forward, as needed.

iii. Check each mouse for any visible ailments and that it is not stuck yin any compromising positions (i.e., inside the feeder).

iv. Airflow is within the proper range.

\section{Starting Protocol}

i. Oxymass Windows

ii. Yes 
iii. Use default hardware configuration

iv. File

v. Open experimental configuration

vi. Recent Places (Han/Carson) Pick trusting protocol

vii. Experimental Configuration

viii. Set-up

ix. Data File

x. Recent-2020 January then save

xi. Put in mouse \# and body weight

xii. Done

b. Properties

i. Environment

ii. Change lighting to today's date/Save to controller

iii. Temperature- 22degress start-end then save to controller

c. Calibrate

i. Put yellow tubing in $\mathrm{O} 2$ tank

ii. Turn on $\mathrm{O} 2$

iii. Experiment

iv. Calibrate

v. Start/yes

vi. $\mathrm{OK}$

vii. Once completed Turn off O2- take our yellow cord

d. Changing Drie-rite

i. Goes purple when moist

ii. Take top part out and check if blue/purplish

iii. Change the two connected ( $1^{\text {st }}$ and last)

e. Hit run then start experiment.

\section{Saving Experiment Files}

i. Stopping Experiments:

1. Experiment

2. Stop

3. Yes

4. Exporting Files

5. Export

6. All Subject CSV's

7. Export

8. Experimental Parameters

9. Selected All-Metabolic, Feeding, Activity

10. Next

11. Finish 


\section{Cleaning:}

i. Metal dishes go into dish washer

ii. put water bottles and water lids upside down

iii. run on plastics

iv. $\sim 52$ minutes

v. Autoclave buckets and place foil on top

vi. Gravity settings

b. Cage Cleaning

i. $10 \%$ alconox

ii. $10 \%$ Bleach

iii. $10 \%$ acetic acid

\section{CLAMs Analysis for Carson Lab}

a. Sorting Data

i. Open Raw file in excel

ii. Copy all raw data into a separate tab

iii. Sort for analysis: VO2, VCO2, RER, heat, Feeding, and Activity remove all unnecessary raw data

iv. In the column to the right of $\mathrm{VO} 2$ and $\mathrm{VCO} 2$ convert into $\mathrm{ml} / \mathrm{hr}$

v. In the column to the right of Activity; Analyze Total XY Activity by summing XTOT and YTOT

vi. Remove the first day of analysis as this is when mice are acclimated to the system. Start with the next day starting at 6:00am.

1. Analysis only needs to be completed on the average of the first three days. *Please refer to other completed analysis if confused.

a. In a separate tab copy and paste the $\mathrm{VO} 2$ in $\mathrm{ml} / \mathrm{kg} / \mathrm{hr}$ and $\mathrm{ml} / \mathrm{hr}$.

i. In side-by-side columns copy and paste the $\mathrm{VO} 2$ values for over the first 3 days, each day in one column.

1. For example, all 6am data will be in side-by-side columns. Resulting in one days $24 \mathrm{hrs}$ of measurements in one column, day 2 will be in the second column, and day 3 in the third column.

ii. Average the 3 days of $\mathrm{VO} 2$ for both $\mathrm{ml} / \mathrm{kg} / \mathrm{hr}$ and $\mathrm{ml} / \mathrm{hr}$. This is done by calculating averages for each measurement. Start a new column then take the average of time point output. Therefore, the last column should be the average of each timepoint over a $24 \mathrm{hr}$ time. 
iii. Copy and paste the average $\mathrm{VO} 2 \mathrm{ml} / \mathrm{hr}$ into the cumulative analysis file.

1. Note* if adding data for the first cohort of mice in the CLAMs unit during that time frame make sure the average column is correctly averaging each hour.

a. When mice were placed in the CLAMs system can be found the weekly tracking sheet.

2. The average column should take the average per hour. Please refer to completed cumulative analysis if confused.

3. Note* There should be no 0's in the analysis.

2. Next, normalize the VO2 to lean mass. Lean mass can be found in the CLAMS weekly tracking file.

a. Take the average per hour of the normalized VO2 to lean mass.

b. Note*please refer to completed averaging on the cumulative sheet if confused.

3. Complete all steps in vi for $\mathrm{VCO} 2$ and heat.

a. Note* if VO2 averages per hour are correct. Copy and paste the average columns to $\mathrm{VCO} 2$ and heat tabs. Then input the VCO2 and heat data into the spot where VO2 was. This will automatically update the averages per hour for totals and normalized values.

4. Complete steps in vi except normalizing to lean mass for RER.

5. Complete steps in vi except normalizing to lean mass and averaging each hour for XY activity.

a. The averaging per hour needs to be changed to sum per hour in the cumulative file. Do not average each hour. 


\section{ECHO MRI}

Get red tubes from TSRB shared lab - head to animal facilities

Calibrate machine $\mathrm{w} /$ sunflower oil (tube is in the room) for $\sim 20 \mathrm{~min}$

Put mouse in larger tube and use other tube to push mouse to the end, so it is

immobile

New file à start scan and enter weight

Label mouse \#, weight "okay" wait for scan to complete

Record lean and fat mass

When finished click ok and turn machine off

\section{Run to Fatigue}

1. Acclimate mice to the treadmill by placing mice in the lanes $10-15$ minutes prior to the start of the warm-up

2. Run the warm-up protocol $5 \mathrm{~min}$ at $5 \mathrm{~m} / \mathrm{min}, 5 \mathrm{~min}$ at $10 \mathrm{~m} / \mathrm{min}, 5 \mathrm{~min}$ at $15 \mathrm{~m} / \mathrm{min}$

3. Start the fatigue test running mice at $20 \mathrm{~m} / \mathrm{min}$

5 . Use gentle hand prodding to keep mice running

6 . Fatigue will be defined as the time at which mice are no longer able or willing to keep up with the treadmill despite gentle hand prodding for a period of $1 \mathrm{~min}$. 7. Clean the treadmill and the area around it with a sponge or paper towels.

Sweep the floor around the treadmills. There should be no feces on the treadmill, the treadmill cart, or the floor when you leave 


\section{Cell Culture}

General: Check and maintain cleanliness of cell culture room

- Each month add fresh ddH2O to water bath

- Add 1-2 drops conditioner every 2 wks

- When vacuum container fills, empty down sink with bleach; add small amount of bleach to container and reattach

- Always wear a lab coat and gloves when working with cells

- Spray hands and all equipment with $70 \%$ EtOH before working with cells to avoid contamination

- Check on cells daily

Growth Media (GM) (store $4^{\circ} \mathrm{C}$ ):

1. Pre warm in water bath $@ 36^{\circ} \mathrm{C}$ :

a. DMEM (Gibco cat\#11995-065, high glucose, 500ml) (new bottle)

b. Fetal Bovine Serum (FBS)

c. Penicillin/Streptomycin cocktail

2. Remove $55 \mathrm{ml}$ DMEM $\rightarrow 50 \mathrm{ml}$ tubes

3. Add $50 \mathrm{ml}$ FBS (10\%)

4. Add $5 \mathrm{ml} \mathrm{Pen} / \operatorname{Strep~(1\% )}$

\section{Differentiation Media (DM) (store $\left.4^{\circ} \mathrm{C}\right)$ :}

1. Pre warm in water bath $@ 36^{\circ} \mathrm{C}$ :

a. DMEM (Gibco cat\#11995-065, high glucose) (new bottle)

b. Horse Serum

c. Penicillin/Streptomycin cocktail

2. Remove $15 \mathrm{ml}$ DMEM $\rightarrow 50 \mathrm{ml}$ tube (place in $4^{\circ} \mathrm{C}$, can combine once you get enough for complete media)

3. Add $10 \mathrm{ml}$ HS (2\%)

4. Add $5 \mathrm{ml} \mathrm{Pen/Strep} \mathrm{(1 \% )}$

5. To be added once myoblasts reach $85-90 \%$ confluency - Day 0 differentiation once added.

a. day 3-4 tubes start to form, fully formed \& contractile tubes $\sim$ day 5-7

Media Change (every 2 days - if you wait too long, media becomes yellow and acidic and can affect cell growth \& environment)

1. Turn on water bath to $36^{\circ} \mathrm{C} \&$ take out required equipment (clean everything with $70 \%$ EtOH before placing into hood)

2. Warm media and invert or pipette up and down to mix

3. Aspirate old media from plate with glass Pasteur pipette tip

4. Add $\sim 1 \mathrm{ml} /$ well of autoclaved sterile PBS to rinse (wash when switching from GM to DM, or when adding a treatment)

5. Aspirate PBS 
6. Add fresh media
a. 6 well plates: $3 \mathrm{ml} / \mathrm{well}$
b. Flasks: $15 \mathrm{ml}$
c. Petri Dish: $10 \mathrm{ml}$
d. $150 \mathrm{~mm}$ Dish: $25 \mathrm{ml}$

7. Place plate back in incubator

\section{C2C12 Myotube formation}

- C2C12 myoblasts are plated in Type-1 collagen (Advanced Biomatrix \#5056) coated 6-well plates at 1.0x10 in GM (3ml per well)

- After 48hrs (85\% confluence), cells are washed with PBS and placed in Differentiation Media (DM) (DMEM, 2\% Horse serum, 1\% Pen/Strep)

- NOTE: Never let C2C12 myoblasts get to $100 \%$ confluence - this will alter the phenotype and reduce differentiation potential down the road

- DM is changed every $48 \mathrm{hrs}$

- Myotubes begin to form around day 3 of differentiation, contractile properties are visible by Day 5-6 of differentiation

\section{CELL HARVEST}

1. Harvest cells to either freeze back, add to new plate, or for mouse tumor cell injection when confluent (85-95\%)

a. NOTE: Never let $\mathrm{C} 2 \mathrm{C} 12$ myoblasts get to $100 \%$ confluence - this will alter the phenotype and reduce differentiation potential down the road

2. Spray everything going under hood with $70 \% \mathrm{EtOH}$

3. Pre warm in water bath $@ 36^{\circ} \mathrm{C}$ :
a. Trypsin
b. Freeze back media
c. Growth media
d. PBS (room temp)

4. Aspirate old media

5. Rinse with PBS, and then aspirate - Growth media inactivates trypsin, must perform this step to detach cells

6. Add 1-3ml trypsin (larger volume for larger flasks, just enough to cover the bottom, should never have to use $>5 \mathrm{ml})$

7. Place in incubator $(\sim 3-5 \mathrm{~min})$

8. Check under scope to ensure cells detach

9. Stop trypsin activity by adding GM $\sim 3-5 \mathrm{ml}$

10. Label $15 \mathrm{ml}$ tubes

11. Remove trypsin $+\mathrm{GM} \mathrm{w} /$ serological pipette into $15 \mathrm{ml}$ tube

12. Spin down at $\mathbf{4 0 0 0} \mathbf{~ r p m} \mathbf{5} \mathbf{~ m i n}$

13. Carefully aspirate media from cell pellet

14. Add $1 \mathrm{ml}$ fresh GM (if replating) or freezing media (if saving back), or PBS (injection) and resuspend pellet by pipetting up and down 
15. Count Cells - NOTE: resuspend cells in GM for counting, then spin down the appropriate \# of cells and resuspend PBS/freeze media if using for injection or to freeze back, if cells are kept in PBS or the DMSO freezing solution too long they will die:

a. Add $10 \mathrm{ul}$ Trypan Blue (TB) $+10 \mathrm{ul}$ cell suspension to $1.5 \mathrm{ml}$ tube

b. Add $10 \mathrm{ul}$ of TB+cells to cell counting slide (countess), insert into machine \& record total and \# alive

16. Calculate dilution:

a. $\boldsymbol{C 1} \mathbf{V 1}=\boldsymbol{C} \mathbf{2} \mathbf{V} \mathbf{2}$ to determine amt cell suspension to add to new flask/cryo tube

$\boldsymbol{C 1}=\#$ cells alive from countess readout

VI = unknown; volume of cell suspension to add to each plate

$\boldsymbol{C 2}=$ final/desired concentration

$1 \times 10^{\wedge} 6$ cells $/ \mathrm{ml}$ for large flasks

$250,000 \mathrm{cells} / \mathrm{ml} /$ well for 6 well plates

$V 2=1 \mathrm{ml}$ (volume of cell suspension in $15 \mathrm{ml}$ tube)

17. For injection:

a. Determine $\mathrm{ml}$ necessary for $1,000,000$ [cell] injection

b. Make 1ml PBS LLC cell solution

c. Ex: $1.0 \times 1 \times 10^{7}$ cells in $1 \mathrm{ml}=0.1 \mathrm{ml}$ injection for 10 mice

18. Add V1 to plate or cryo tube

19. Plate: add growth media and place in incubator

a. Follow steps for media change and check confluency of cells daily

20. Cryo tube: place in Mr. Freeze -20 overnight $\rightarrow$ Mr. Freeze $-80 \rightarrow \mathrm{LN}_{2} \operatorname{tank}$

\section{Collagen Coating: (protocol is adapted from collagen website - Advanced}

\section{Biomatrix)}

Collagen coat 6-well plates for $\mathrm{C2C12}$ myotubes

1. Take out materials:

a. Collagen Rat Tail Type $1\left(4^{\circ} \mathrm{C}\right) @ 3.9 \mathrm{mg} / \mathrm{ml}$

b. Acetic Acid

c. Sterile ddH2O

2. Each 6 well plate $=1 \mathrm{ml} / \mathrm{well}$

3. Stretcher $=3 \mathrm{ml}$

4. Count total wells to coat

a. Ex: 18 plates $x 6$ wells $=108$

$b$. $\rightarrow$ round up $110 \mathrm{ml}$ final desired volume

5. Determine amt collagen to add
a. $3.9 \mathrm{mg} / \mathrm{ml}$ collagen
b. $C l V l=C 2 V 2$
c. $\mathrm{C} 1=3.9 \mathrm{mg} / \mathrm{ml}$
d. $\mathrm{V} 1=\mathrm{X}$
e. $\mathrm{C} 2=0.1 \mathrm{mg} / \mathrm{ml}$
f. $\quad V 2=110 \mathrm{ml}$
g. $(3.9)(x)=(0.1)(110)$ 
h. $x=2.8 \mathrm{ml}$ collagen

6. Determine ddH2O to add:

a. $\mathrm{V} 2-\mathrm{V} 1=\mathrm{ddH} 2 \mathrm{O} \mathrm{ml}$

b. $110-2.8=107.2 \mathrm{ml}$

7. Determine amt acetic acid to add:

a. Want $0.1 \%$ of ddH2O (NOT TOTAL VOLUME)

b. $\mathrm{ddH} 2 \mathrm{O} \mathrm{ml} * 0.001=\mathrm{ml}$ acetic acid

c. $107.2 * 0.001=0.1072 \mathrm{ml} * 1000=107.2$ ul acetic acid

8. Optional: run collagen solution through a filter (Nalgene 20micron)

9. Add $1 \mathrm{ml}$ of final solution to each well

10. Let sit for $\sim 2 \mathrm{hrs}$ under UV

11. Can store plates in fridge up to $\sim 2$ weeks

\section{Harvest Experimental 6-well plates \\ Protocol: Keep everything ice cold}

1. When experiment is complete aspirate media and rinse twice with ice cold PBS, aspirate, and place plates on ice to harvest.

2. Add 100ul RIPA cocktail to each well, scrape cells with blue cell scraper, transfer to $1.5 \mathrm{ml}$ conical tube [see example below]

a. $100 u l$ used in the lab and results in $\sim 1.0-3.5 \mathrm{ug} / \mathrm{ul}$ protein (acquired from Bradford)

3. Aliquot $1 \mathrm{ml} \mathrm{RIPA}$ (thermo cat \# 89900) into $1.5 \mathrm{ml}$ conical tube - place on ice

4. Immediately before scraping plate add the following to RIPA buffer:

a. Add 10ul protease and phosphatase inhibitor cocktail (thermo cat \# 78440)

b. Add 10ul EDTA

5. NOTE: ONE 6-well plate will require 600ul RIPA (+ 6ul protease + 6ul EDTA) $\rightarrow$ calculate total buffer needed before making cocktail and adjust ratios accordingly

6. Centrifuge scraped cells in RIPA cocktail at $14,000 \mathrm{rpm}$ for $15 \mathrm{~min}$ at $4{ }^{\circ} \mathrm{C}$ to remove cell debris (pellet gets thrown away, transfer supernatant to new tube, add date and label sample).

7. Store supernatant at $-80 \mathrm{C}$

\section{Conditioned Media Collection}

1. $2.0 \times 10^{6} \mathrm{C} 26$ or LLC cells are seeded in $100 \mathrm{~mm}$ tissue culture treated plates in $12 \mathrm{ml}$ Growth Media (GM) (gibco DMEM cat\#11995-065; 10\% FBS; 1\% Pen/Strep cocktail)

2. Conditioned media is collected $48 \mathrm{hrs}$ ( $\sim 90 \%$ confluence) post cell seeding and spun down at $3000 \mathrm{rpm}$ for $5 \mathrm{~min}$ to remove cell debris

3. Cells on the plate were pelleted and counted via trypan blue exclusion test to ensure an equivalent number of cells on the plate, with a final density averaging from $7.0-9.0 \times 10^{6}$ cells per culture dish

4. Conditioned media is stored in aliquots at $-20^{\circ} \mathrm{C}$ (Freeze thaw cycles may affect media components, so only one freeze thaw cycle is ideal) 
5. At time of experiment, conditioned media aliquot is warmed in warm water bath

\section{Conditioned Media Dosing:}

- Conditioned media is diluted with 50\% serum-free DMEM for a final serum concentration of $5 \%$ FBS

- $\quad 50 \% \mathrm{CM}$ was chosen as it has been shown to produce significant myotube atrophy (Pin et al., 2018; Zhong et al., 2019). 


\section{Tissue Homogenization for Protein}

1. Cut and weigh a small portion of frozen tissue in liquid nitrogen $(\sim 20-35 \mathrm{mg})$

2. Add beads to locking homogenization tubes (label with EtOH proof marker on top and side of tube)

3. Calculate total Mueller \& Diluent Buffer based on tissue weights

a. Mueller $=10 \mathrm{X}$ mg tissue

b. Diluent $=1 / 2$ Mueller

c. Example: $20 \mathrm{mg}$ tissue $=200 \mathrm{ul}$ Mueller $+100 \mathrm{ul}$ Diluent

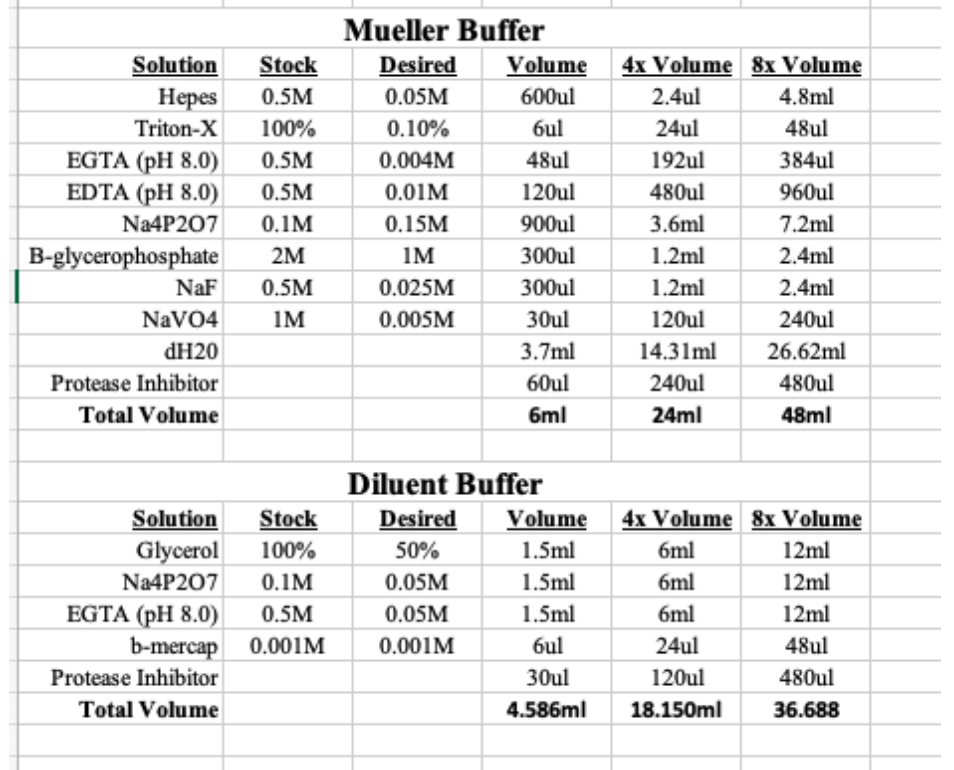

4. Add Mueller Buffer to tubes with beads \& place on ice

5. Add frozen tissue to Mueller buffer tube

6. Freeze at -20C overnight

7. Remove from $-20 \mathrm{C}$ and thaw on ice for $\sim 20 \mathrm{~min}$

8. Bring samples to bead homogenizer on the $4^{\text {th }}$ floor of TSRB

a. Homogenize at $30 \mathrm{~Hz}$ for $3 \mathrm{~min}$ à ice for $\sim 5 \mathrm{~min}$ '

b. If clumps remain, repeat in 2 min intervals

9. Centrifuge homogenized samples at $15000 \mathrm{rpm}$ for $15 \mathrm{~min}$.

10. Add diluent buffer to a new $1.5 \mathrm{ml}$ conical tube and place on ice

11. Remove supernatant from centrifuged samples and add to tubes with diluent buffer

12. Quantify protein via Bradford Protein Assay

13. Freeze samples at $-80 \mathrm{C}$ for long term storage

STOCK Solutions with Importance

HEPES - Acros Organics (fisher) \#172571000; soluble in H2O

EGTA (pH 8.0) - Sigma \#E4378-25g; soluble in $\mathrm{dH} 2 \mathrm{O}$ at basic $\mathrm{pH}$

EDTA (pH 8.0) - Sigma \#ED2SS-500g

$\mathrm{Na}_{4} \mathrm{O}_{7} \mathbf{P}_{2}$ (Sodium pyrophosphate tetrabasic) - Sigma \#P8010-500g; H2O soluble

b-Glycerophosphate - Sigma \#G9422-10g; H2O soluble

NaF (Sodium Fluoride) - Sigma \#S7920-100g 
$\mathrm{NaVO}_{4}$ (Sodium Orthovanadate) - Sigma S6508-10g H2O soluble

Protease Inhibitor Cocktail (PIC) - Sigma \#P8340-5ml; DMSO solution

\section{Genotyping}

Scruff \& cut small piece from end of tail, place in pre-labeled $1.5 \mathrm{ml}$ tube Add 200 ul tailing buffer (enough to cover tail completely) - use molecular grade $\mathrm{H} 2 \mathrm{O}$ to avoid DNA contamination

a. For master mix, make extra (add $~ 2)$

b. i.e., 12 tails $+2=14$ à $14 * 200=2800 \mathrm{ml}$

c. Make $3 \mathrm{ml}$ tailing buffer and add $75 \mathrm{ul}$ proteinase $\mathrm{K}$

Place in water bath overnight ( $\sim 8$ hrs minimum)

Remove \& vortex then centrifuge to pull tail residue to the bottom of the tube

Put on heat block at $92^{\circ} \mathrm{C}$ for 10 min to stop proteinase $\mathrm{K}$ activity

Remove \& vortex

Spin down for 30-60s in mini centrifuge

Use for PCR or store in $4^{\circ} \mathrm{C}$ (this is the DNA)

\section{Genotyping PCR}

Take out PCR tubes and label

Determine genotypes and make a master mix (formula sheet on excel doc)

a. Sample Sets: B6 = control; Min; AMPKa1; AMPKa2; HSA; GP130

i. Flox $=$ single band, Flox $+=$ double band

b. Master Mix $=$ molecular grade $\mathrm{dH} 2 \mathrm{O}$ or Rnase free $\mathrm{dH} 20$, GoTaq, forward \& reverse primers

Add 23 ul master mix to each tube

Add 3 ul DNA

Run PCR reaction

When reaction has $\sim 30$ min left, cast gel

2\% Agarose Gel

c. $100 \mathrm{ml} 1 \mathrm{X}$ TAE

d. $2 \mathrm{~g}$ agarose

e. 2 ul Midori Green

Mix and microwave in 30 second intervals until boiling and clear (at least 30 seconds intervals) Swirl thoroughly.

Pour into mold (make sure clear casing is inserted) and place combs for lanes

Wait $\sim 30$ min for gel to cool

Remove gel and place in PCR electrophoresis, add $\sim 300 \mathrm{ml} 1 \mathrm{XTAE}$ to cover/fill

Once reactions are complete pipette sample into each well

f. Min RXN: 10ul

g. HSA RXN: 9ul

h. gp130 RXN: 10ul

i. AMPK alpha 1 and alpha 2 RXN: 8 ul

Add 8 ul DNA ladder 4:1:1 ratio

j. $16 \mathrm{ul} \mathrm{dH} 2 \mathrm{O}$ 
k. 4 ul DNA ladder

1. 4 ul $6 \mathrm{X}$ tracking dye

Run gel for $30 \mathrm{~min}$ at 100 volts (check $5 \mathrm{~min}$ in to make sure it is running)

Remove gel place on plastic divider

Image on nucleic acids.

\section{Tailing for Genotyping}

Scruff \& cut small piece from end of tail, place in pre-labeled $1.5 \mathrm{ml}$ tube Add 200ul of master mix tailing buffer to each $1.5 \mathrm{ml}$ tube $\mathrm{w} /$ tail (make sure tail is in solution at the bottom)

d. Master mix tailing buffer $-200 \mathrm{ul}$ tailing buffer $+5 \mathrm{ul}$ Proteinase $\mathrm{K}$

e. Example: i.e., 12 tails $\rightarrow 12 * 200=2400 \mathrm{ml}$

Place in water bath overnight @56degree ( $~ 8$ hrs minimum)

Next Morning - Remove \& vortex then centrifuge to pull tail residue to the bottom of the tube

Put on heat block at $95^{\circ} \mathrm{C}$ for 10 min to stop proteinase $\mathrm{K}$ activity

Remove \& vortex

Spin down for 30-60s in mini centrifuge

Use for PCR or store in $4^{\circ} \mathrm{C}$

\begin{tabular}{|l|l|l|}
\hline Final Concentration & Volume & Stock Solution \\
\hline 10nM Trish-HCL, $\mathrm{pH} 8.3$ & $5 \mathrm{~mL}$ & $1.0 \mathrm{M}$ \\
\hline $50 \mathrm{nM} \mathrm{KCL}$ & $25 \mathrm{~mL}$ & $1.0 \mathrm{M}$ \\
\hline $2.5 \mathrm{nM} \mathrm{MgCl} 2,6 \mathrm{H} 20$ & $1.25 \mathrm{~mL}$ & $1.0 \mathrm{M}$ \\
\hline $0.45 \% \mathrm{NP}-40$ & $2.25 \mathrm{~mL}$ & - \\
\hline $0.35 \%$ Tween 20 & $2.25 \mathrm{~mL}$ & - \\
\hline $\begin{array}{l}\text { Molecular Biology Grade } \\
\text { dH20 }\end{array}$ & Volume to $500 \mathrm{~mL}$ & \\
\hline
\end{tabular}

Tailing Buffer - Sterilize all stock solution prior to making tailing buffer.

Store stock solutions at room temp and tailing buffer at 4 degrees. 


\section{Genotyping PCR}

Take out PCR tubes out and label

Make a master mix

m. Master Mix $=$ molecular grade $\mathrm{dH} 2 \mathrm{O}$ or Rnase free $\mathrm{dH} 20$, GoTaq, forward $\&$ reverse primers (see master mix volumes below and an example far right column)
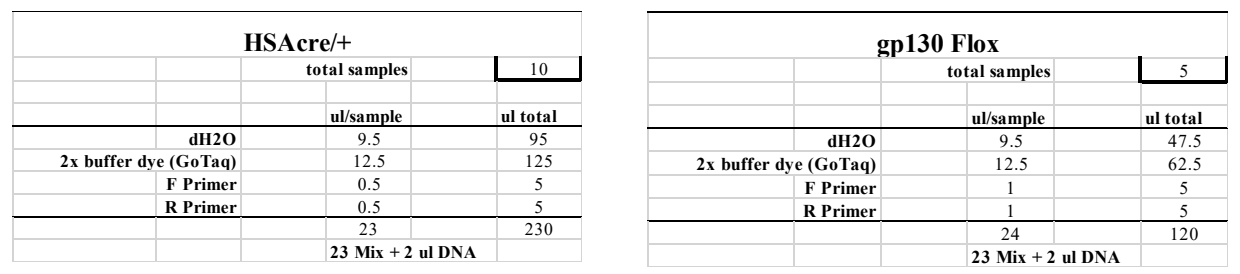

Add 23 ul master mix to each tube Add 2 ul DNA

Run PCR reaction (see below)

$$
\begin{gathered}
\text { Thermocycler Protocol } \\
\text { Gp130 } \\
\text { Stage 1 (1x): } 95^{\circ} \mathrm{C} 5 \mathrm{~min} \\
\text { Stage 2 (4x): } 95^{\circ} \mathrm{C} 30 \mathrm{sec} \\
65^{\circ} \mathrm{C} 1 \mathrm{~min} \\
72^{\circ} \mathrm{C} 30 \mathrm{sec} \\
60^{\circ} \mathrm{C} 1 \mathrm{~min} \\
72^{\circ} \mathrm{C} 30 \mathrm{sec} \\
\text { Stage 3 (34x): } 95^{\circ} \mathrm{C} 30 \mathrm{sec} \\
\text { Stage } 4(1 \mathrm{x}): 72^{\circ} \mathrm{C} 10 \mathrm{sec} \\
\text { Stage 5 (infinity): } 4{ }^{\circ} \mathrm{C} \\
\text { Thermocycler Protocol } \\
\text { Gp130 } \\
\text { Stage 1 (1x): } 95^{\circ} \mathrm{C} 5 \mathrm{~min} \\
\text { Stage 2 (4x): } 95^{\circ} \mathrm{C} 30 \mathrm{sec} \\
65^{\circ} \mathrm{C} 1 \mathrm{~min} \\
72^{\circ} \mathrm{C} 30 \mathrm{sec} \\
60^{\circ} \mathrm{C} 1 \mathrm{~min}
\end{gathered}
$$

When reaction has $\sim 30$ min left, cast 2\% Agarose Gel
n. $100 \mathrm{ml} 1 \mathrm{X}$ TAE
o. 2 g agarose
p. 2 ul Midori Green

\section{Thermocycler Protocol HSA-MCM}

Stage $1(1 \mathrm{x}): 95^{\circ} \mathrm{C} 3 \mathrm{~min}$ Stage $2(34 \mathrm{x}): 95^{\circ} \mathrm{C} 20 \mathrm{sec}$ $58^{\circ} \mathrm{C} 20 \mathrm{sec}$ $72^{\circ} \mathrm{C} 30 \mathrm{sec}$

Stage $3(1 \mathrm{x}): 72^{\circ} \mathrm{C} 10 \mathrm{~min}$

Stage 4 (infinity): $4^{\circ} \mathrm{C}$

\section{Thermocycler Protocol}

HSA-MCM

Stage 1 (1x): $95^{\circ} \mathrm{C} 3 \mathrm{~min}$

Stage $2(34 \mathrm{x}): 95^{\circ} \mathrm{C} 20 \mathrm{sec}$

$$
58^{\circ} \mathrm{C} 20 \mathrm{sec}
$$$$
72^{\circ} \mathrm{C} 30 \mathrm{sec}
$$

Stage $3(1 \mathrm{x}): 72^{\circ} \mathrm{C} 10 \mathrm{~min}$

gel

Stage 4 (infinity): $4^{\circ} \mathrm{C}$

Mix and microwave in 30 second intervals until boiling and clear (at least 15-30 second intervals) Swirl thoroughly.

Pour into mold (make sure clear casing is inserted) and place combs for lanes Wait $\sim 20 \mathrm{~min}$ for gel to cool Remove gel and place in PCR electrophoresis, add 300ml 1XTAE to cover/fill Once reactions are complete pipette sample into each well

q. HSA-MCM RXN: 7ul

r. gp130 RXN: 8ul 
Add 8 ul DNA ladder 4:1:1 ratio
s. $\quad 16 \mathrm{ul} \mathrm{dH} 2 \mathrm{O}$
t. 4 ul DNA ladder
u. 4 ul $6 X$ tracking dye

Run gel for $30 \mathrm{~min}$ at 100 volts (check $5 \mathrm{~min}$ in to make sure it is running) Image (example below)

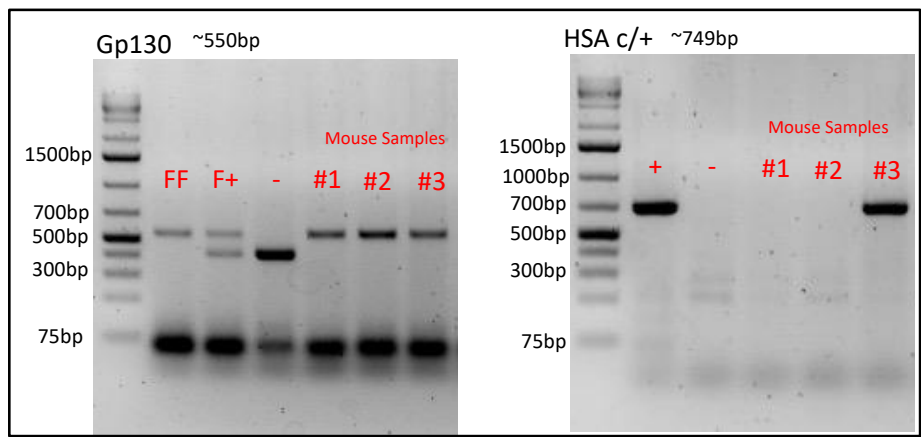

$\square$ Item Name gp130 Flox Forward

Product 25 nmole DNA Oligo Length $20 \quad$ Lot\#

Purification Standard Desalting Documents 因Spec Sheet 因ESI

Reference Number $293024112 \quad$ Expected Ship Date 2/5/2021

Delivery Plant North Carolina

Sequence GGCTITCCTCTGGTTCTTG

$\square$ Item Name gp130 flox reverse

Product 25 nmole DNA Oligo

Purification Standard Desalting Documents 园 Spec Sheet 园ESI

Reference Number $293024113 \quad$ Expected Ship Date 2/5/2021

Delivery Plant North Carolina

Sequence ACGTCACAGAGCTGAGTGATGCAG

Primers: Purchased through Integrated DNA Technologies - 25nmole DNA Oligo

HSAc/+ MCM Forward - See image below

HSAc/+ MCM Reverse - See image below

gp130 Forward - See image below

gp130 Reverse - See image below

Primers: Purchased through Integrated DNA Technologies - 25nmole DNA Oligo

HSAc/+ MCM Forward - See image below

HSAc/+ MCM Reverse - See image below

gp130 Forward - See image below

gp130 Reverse - See image below 


\section{Blood Glucose}

1. Immediately prior to ad libitum access to a food pellet, post food pellet, and at sacrifice mice had their tail snipped.

2. $\sim 1 \mathrm{~cm}$ of tail was snipped and blood was wiped with a kim wipe.

3. Milk the tail and analyze blood using the readily available glucometer (Contour Next, Parsippany, NJ).

\section{Plasma Insulin}

1. Immediately prior to ad libitum access to a food pellet, post food pellet, and at sacrifice mice had their tail snipped.

2. Snip $\sim 1 \mathrm{~cm}$ of tail was snipped and wipe blood with a kim wipe.

3. Milk the tail to get $\sim 50 \mathrm{ul}$ in a heparinized capillary tube.

4. Place on ice till centrifuged.

5. Centrifuged $\left(10,000 \mathrm{x}\right.$ g for $10 \mathrm{~min}$ at $\left.4^{\circ} \mathrm{C}\right)$.

6. The supernatant was removed, and plasma insulin concentrations were determined using a two-site enzyme immunoassay assay kit according to the manufacturer's instructions (Catalog \#10-1247, Mercodia, Uppsala, Sweden).

7. Store in -20 till analysis .

\section{Western Blot}

Determine which size gel and the appropriate $\%$ for protein of interest

$$
\text { ○ - MW, } \% \text { gel }
$$

When making SDS wear mask and goggles (cancerous)

- Mini gels need $8 \mathrm{~mL}$ per 1 gel

$\circ$ Midi (large) gels need $10 \mathrm{~mL}$ per 1 gel

- Stacking $3 \mathrm{~mL}$ per 1 gel

Set up casting station and take out materials

○ ddH20 - autoclaved water

- $30 \%$ acrylamide -4 degree

○ $1.5 \mathrm{M}$ Tris ph. 8.8 - RT

○ $10 \%$ SDS - RT

○ $10 \%$ APS - -20degree

- TMED - RT

Mix ingredients together (swirl after adding each ingredient), add TMED last Pipette thoroughly mixed materials into casting station.

Wait $\sim 2$ minutes; add methanol to top of gel while it sets $\sim 60 \mathrm{~min}$ Remove methanol w/ vacuum attachment Make stacking portion of the gel (always make 6\% stacking) Mix, add TMED last, and pipette into gel 
Add comb for wells immediately after pipetting mixed ingredients into the casting station (check the number of wells needed)

Let set $\sim 60$ minutes

\section{Running Gels}

Determine amount of sample to add based on Bradford assay, protein, \& marker of interest (15-50 ug) - check antibody sheet for reference

Prepare samples (turn on heat block)

a. Label tubes

b. Add half of loading volume sample 2 XSDS (2\%), 1/3 for 4XSDS stored $20^{\circ} \mathrm{C}$

i. Use $4 \mathrm{x}$ if sample volume is too large for wells.

c. Add sample to $1.5 \mathrm{~mL}$ tube then vortex

d. Samples can be prepped the day before and stored in $-80^{\circ} \mathrm{C}$

Place samples on heat block for $5 \mathrm{~min}$ at $92-95^{\circ} \mathrm{C}$

Vortex and spin down - NO BUBBLES

Place on ice till use

Running Gels:

e. Set up casting stations for a run. Place gel in tank (align the charges in toward middle)

f. Add 1X SDS running buffer

i. Dilute $10 \mathrm{X}$ stock SDS running buffer to $1 \mathrm{X}$ needs to mix for $\sim 10$

minutes if not the running current will be off, and gel will not run straight.

1. Determining Volume needed calculation
a. $\mathrm{C} 1 \mathrm{~V} 2=\mathrm{C} 2 \mathrm{~V} 2$
b. $(10 \mathrm{X})($ unknown volume $)=(1 \mathrm{x})(1000 \mathrm{~mL})$
c. $(10 \mathrm{X})($ unknown volume $)=(1000 \mathrm{~mL})$
d. $($ Unknown volume $)=\left(1000 \mathrm{~mL}^{*} 1 \mathrm{X}\right) /(10 \mathrm{X})$
e. Unknown volume $=100 \mathrm{ml}$ of $10 \mathrm{X}$ stock SDS
f. Need $1 \mathrm{~L}$ therefore your volume up to $1 \mathrm{~L}$
g. $100 \mathrm{~mL}$ of $10 X$ SDS and $900 \mathrm{~mL}$ of $\mathrm{dH20}$

g. Pipette samples and ladder (5 ul) into wells

h. Note* if samples are over $20 \mathrm{ul}$-pipette $20 \mathrm{ul}$ of sample into well, run for 2 minutes and then pipetting the rest of the sample into the well

i. Be careful when pipetting samples $\&$ avoid bubbles

i. Bubble will pushout samples from wells.

j. Fill tank with 1X SDS running buffer so that gels are covered \& place lid on

k. Run at 120 volts until protein is through stacking, then up it to 140 volts ( $\sim 90 \mathrm{~min})$

Run is finished when ladder has a nice spread and protein is near bottom of gel - or protein of interest are in the middle of the gel.

\section{Transferring Gels:}


Make transfer buffer (only need $50 \mathrm{ml}$ methanol for 1 or $2 \mathrm{~L}$ ) and stir with magnetic stir bar.

Cut PVDF membrane - do not touch with fingers as oil will destroy the membrane. Only use forceps and tweezers to touch membrane.

\section{Let membrane sit in methanol for 5 min on shaker}

\section{Wet Transfer Midi Gels (large gels):}

m. Make sandwich: Black cassette side; sponge; filter paper; gel; membrane; filter paper; sponge; red side cassette (close) roll after each step to remove bubbles

i. * Membrane paper should not be larger than the gel. This allows for air bubbles to go underneath the gel.

n. Place in transfer tank (2L transfer buffer) red to red, black to black Transfer runs black to red.

o. Add ice pack in back and stir bar to keep TB moving

p. Run at 1.8Amps for 90min (add ice to bucket so it does not overheat)

Probing:

q. Take out cassette and follow steps 23 on

r. After transfer is complete remove lid, filter paper, and gel (save filter paper, clean and dry flat in clean space)

s. Carefully remove membrane and cut triangle at ladder depending on protein of interest, cut in corner of first well for easy reading

t. Add membrane to antibody box

u. Ponceau stain to check transfer à add $10 \%$ of $0.1 \%$ ponceau with $\mathrm{dH} 2 \mathrm{O}$ and let rock for $\sim 15 \mathrm{~min}$

v. Scan ponceau membrane and save - make sure equal loading. If not, then trash and start over.

w. Place membrane back in the box and wash with TBST $3 x$ until pink is gone

$x$. For blocking/primary and secondary steps - Reference antibody sheet.

y. Between blocking and primary - wash once for 5-10minutes with $1 \mathrm{X}$ TBST

z. Between primary and secondary - wash 3 times for 5-10 minutes with $1 \mathrm{X}$ TBST

aa. Between secondary and ECL - wash 3 times for 5-10 minutes with $1 \mathrm{X}$ TBST

\section{Imaging:}

bb. Place membrane on plastic cover \& pipette ECL reagent (Prometheus usually) for imaging

cc. ratio is $1: 1$ of $\mathrm{A}: \mathrm{B}, 1 \mathrm{~mL}$ per membrane - make sure entire membrane is covered.

Leave reagent on membrane 1-2 min for total proteins and 3-5 minutes for phosphorylation proteins, then aspirate off 
Cover membrane with the plastic then remove excess ECL reagent with KIM wipes and bring down to image

Open CARSON 3356 à Chemi blots à Smart exposure to determine exposure time More options à routines à signal accumulation à $\sim 10$ exposures and set start/end time according to smart exposure to get a good image à options à membrane overlay Save image \& membrane overlay to flash drive ash membrane in TBST and either Ponceau stain and save back, or add stripping buffer for next antibody (i.e., total AMPK)

Start text, $1^{\text {st }}$-Level Head, or table/figure on this line. 


\section{VITA}

Brittany was born in Greenwood, Indiana in 1990. She was raised in Greenwood, Indiana, where she graduated from Whiteland High School in 2009. Brittany's research interest involves understanding the molecular mechanisms that drive muscle wasting with disease and treatments. Brittany's long-term research goal is to elucidate multimodal therapeutic approaches that target to reverse or treat the systemic and local muscle environmental disruptions that occur during muscle wasting. Brittany's academic training and research experience have provided her with an exceptional background in human physiology and molecular biology. Her initial experience with research was as an undergraduate student, where she participated as a subject in a research study. Following completion of her bachelor's degree in 2014, Brittany began her graduate school training at the University of Mississippi under the mentorship of Dr. Jeremy Loenneke, examining the impact of external load on skeletal muscle mass and function with and without blood flow restriction in human subjects. Upon completion of her Master's degree, she moved to the University of South Carolina and began research under the mentorship of Dr. James Carson. Brittany undertook projects utilizing pre-clinical models to examine the effects of diurnal fluctuations of feeding and activity behaviors on skeletal muscle mTORC1 during cancer cachexia progression. During the third year of Brittany's doctoral work, Dr. Carson received the opportunity to become Senior Associate Dean of Research and Graduate Studies in the College of Health Professions at the University of Tennessee Health Science Center (UTHSC) in Memphis. Given her great interest in understanding the molecular mechanisms of muscle wasting with chronic disease; specifically, in models of cancer, she transferred to UTHSC to continue this work. Brittany plans on completing her Doctor of Philosophy in Biomedical Sciences in the Rehabilitation Track in December 2021.

\section{Complete List of Published Work for Brittany Franch}

https://www.ncbi.nlm.nih.gov/myncbi/1bw2SxdcJZDQKP/bibliography/public/ 